\title{
Scanning angle Raman spectroscopy: Investigation of Raman scatter enhancement techniques for chemical analysis
}

\author{
by \\ Matthew W. Meyer \\ the degree of \\ DOCTOR OF PHILOSOPHY \\ Major: Analytical Chemistry \\ Program of Study Committee: \\ Emily A. Smith, Major Professor \\ Robert S. Houk \\ Young Jin Lee \\ Patricia A. Thiel \\ Edward $\mathrm{Yu}$
}

A dissertation submitted to the graduate faculty in partial fulfillment of the requirements for

Iowa State University

Ames, Iowa

2013

Copyright @ Matthew W. Meyer, 2013. All rights reserved. 
Dedicated to my parents, my wife Amanda and our daughter Macall 


\section{TABLE OF CONTENTS}

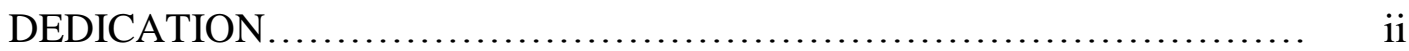

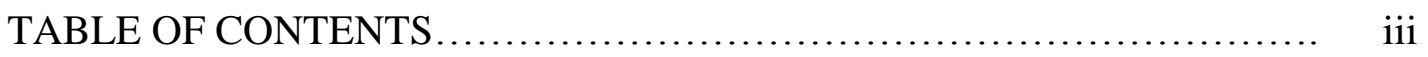

ACKNOWLEDGEMENTS $\ldots \ldots \ldots \ldots \ldots \ldots \ldots \ldots \ldots \ldots \ldots \ldots \ldots \ldots \ldots \ldots \ldots$, vii

DISSERTATION OVERVIEW ...................................... 1

CHAPTER 1: GENERAL INTRODUCTION ............................. 3

Abstract.......................................................... 3

Introduction...................................................... 3

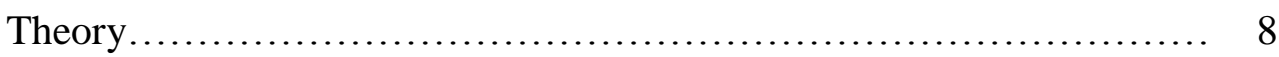

TIR-Raman spectroscopy at a prism interface...................... 18

SPR-Raman spectroscopy at a gold interface $\ldots \ldots \ldots \ldots \ldots \ldots \ldots \ldots \ldots .22$

PWR-Raman spectroscopy at a dielectric interface.................. 23

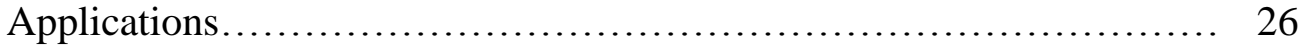

References...................................................... 30

Figures.................................................... 33

CHAPTER 2: SCANNING ANGLE RAMAN SPECTROSCOPY MEASUREMENTS OF THIN POLYMER FILMS FOR THICKNESS AND COMPOSITION ANALYSIS (A paper published in J.of Vib. Spec., 2012, 65, 94-100.)

Abstract......................................................... 41

Introduction...................................................... 42

Experimental............................................. 45

Results and discussion...................................... 47

Conclusions................................................ 54 
Acknowledgement........................................... 55

References......................................................... 56

Figures......................................................... 58

CHAPTER 3: SCANNING ANGLE PLASMON WAVEGUIDE RESONANCE RAMAN SPECTROSCOPY FOR THE ANAYSIS OF THIN POLYSTYRENE FILMS (A paper published in J. of Phys. Chem. C., 2012, 47, 24987-24992.)

Abstract............................................................ 65

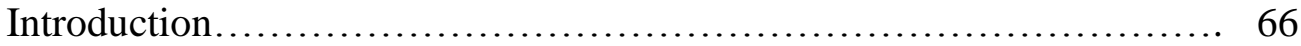

Experimental...................................................... 69

Results and discussion......................................... 72

Conclusions.................................................... 79

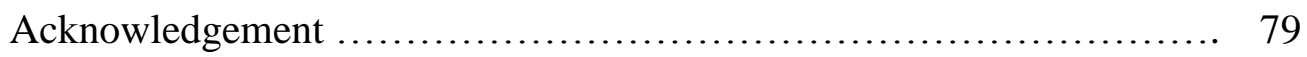

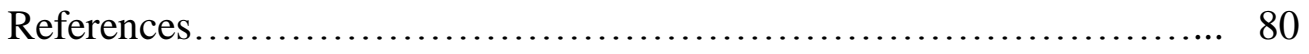

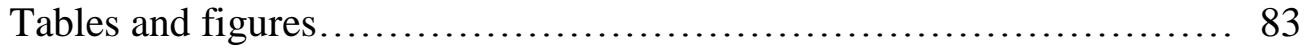

\section{CHAPTER 4: SCANNING ANGLE TOTAL INTERNAL REFLECTION RAMAN}

SPECTROSCOPY OF THIN POLYMER FILMS (A paper published in MRS Online Proc.

Lib., 2013, 1552, doi:10.1557/opl.2013.133.)

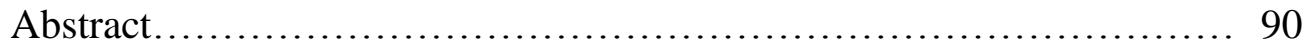

Introduction...................................................... 91

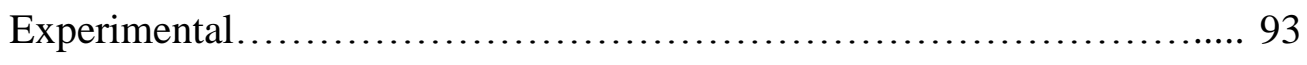

Discussion........................................................ 93

Conclusions....................................................... 97 
Acknowledgement............................................ 98

References...................................................... 99

Figures.......................................................... 100

CHAPTER 5: OPTIMIZATION OF SILVER NANOPARTICLES FOR SURFACE

ENHANCED RAMAN SPECTROSCOPY OF STRUCTURALLY DIVERSE ANALYTES

USING NEAR-INFRARED EXCIATION (A paper published in Analyst, 2011, 17, 3542.)

Abstract......................................................... 104

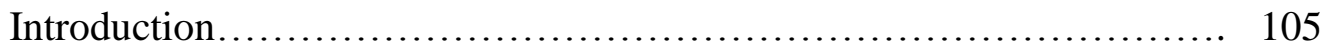

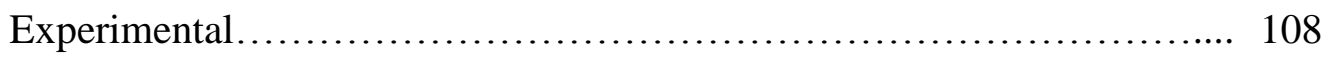

Results and discussion............................................... 111

Conclusions....................................................... 119

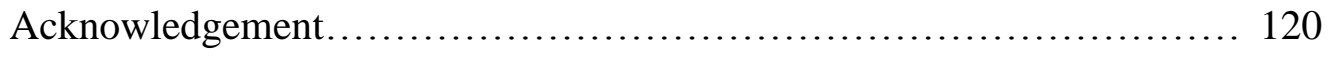

References................................................... 121

Tables and figures................................................ 125

Supplementary information........................................ 133

CHAPTER 6: 1064 NM DISPERSIVE MULTICHANNEL RAMAN SPECTROSCOPY

FOR ANALYSIS OF PLANT LIGNIN (A paper published in Anal. Chim. Acta., 2011, 706, 164-170.)

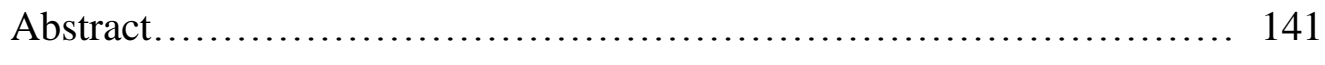

Introduction................................................... 142

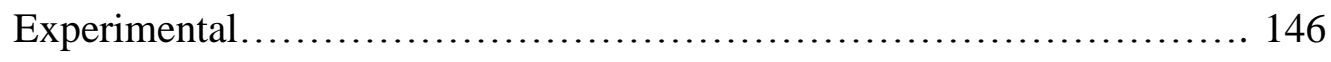

Results and discussion............................................ 149 
Conclusions.................................................... 156

Acknowledgement........................................... 157

References.................................................... 158

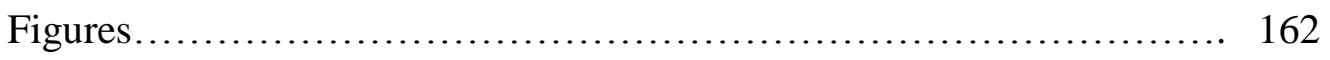

Supplemental information......................................... 169

CHAPTER 7: GENERAL CONCLUSION.................................... 177

APPENDIX: SURFACE ENAHNCED RAMAN SPECTROSCOPY (SERS): RECENT

DEVELOPMENTS IN SERS SUBSTATES AND APPLICATIONS TO SINGLE CELL

ANALYSIS (A section of the review article published in Chem. Rev., 2013,

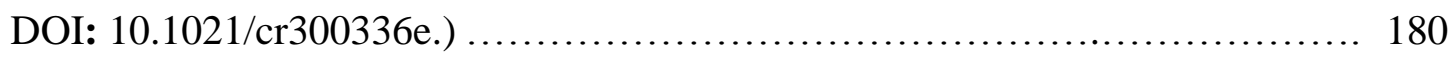

SERS substrates: nanoparticle uptake, tracking, stability and

functionalization.............................................. 182

SERS substrates: nanostars, flowers, clusters, and assemblies............ 186

Detection of small molecules..................................... 187

Detection of cancer cells and treatment efficacy..................... 188

SERS-active substrates: dual imaging mode probes.................... 190

References................................................... 193

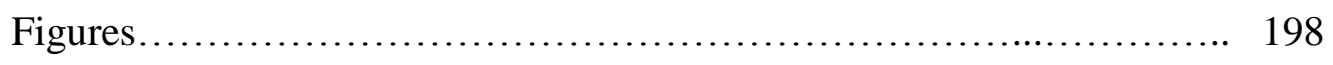




\section{ACKNOWLEDGEMENTS}

Many thanks are due to:

- My supervisor, Professor Emily Smith, with your guidance I have been able to develop into a researcher, scientist and a strategic thinker;

- My committee members for all of their guidance over my graduate school career;

- All of my colleagues over the past years who I've had the privilege to work with and learn from;

○ U.S. Department of Energy through Ames Laboratory for providing the support to perform this research;

- Amanda and my princess Macall.

Additional thanks to anyone that is due thanks, but is not mentioned in the list above.

This work was performed at the Ames Laboratory under contract number DE-AC0207CH11358 with the U.S. Department of Energy. The document number assigned to this thesis is IS-T 3086. 


\section{DISSERTATION OVERVIEW}

Over the past 25 years Raman spectroscopy has transitioned from a technically challenging and time consuming research technique to a valuable and practical method of chemical analysis. During this time span, coined the 'Raman renaissance' by Dr. Richard McCreery, enhancement techniques and improvements to near-infrared detectors have played a significant role in extending the utility of this analytical technique. This thesis outlines advancements in Raman scatter enhancement techniques by applying evanescent fields, standing-waves (waveguides) and surface enhancements to increase the generated mean square electric field, which is directly related to the intensity of Raman scattering. These techniques are accomplished by employing scanning angle Raman spectroscopy (Chapter 24) and surface enhanced Raman spectroscopy (Chapter 5). In Chapter 6, a $1064 \mathrm{~nm}$ multichannel Raman spectrometer is discussed for chemical analysis of lignin. Extending dispersive multichannel Raman spectroscopy to $1064 \mathrm{~nm}$ reduces the fluorescence interference that can mask the weaker Raman scattering. Overall, these techniques help address the major obstacles in Raman spectroscopy for chemical analysis, which include the inherently weak Raman cross section and susceptibility to fluorescence interference.

Chapter 1 is a general introduction to total internal reflection (TIR) Raman spectroscopy and scanning angle (SA) Raman spectroscopy. Brief introductions to surface enhanced Raman spectroscopy and $1064 \mathrm{~nm}$ multichannel Raman spectroscopy are provided in their respective Chapters, 5 and 6. In Chapters 2-4, the utility of scanning angle Raman spectroscopy is put into practice for compositional and thickness measurements of thin polymer films. SA-Raman spectroscopy can be classified as an enhancement technique that 
can be used for measuring interfacial phenomena with chemical specificity. With improvements to modern technology, scanning angle Raman spectroscopy can provide a practical and adaptable technique for applications where surface enhanced Raman spectroscopy (SERS) is impractical. In Chapters 3-4, the total internal reflection Raman spectroscopy configuration is expanded to include surface plasmon resonance and plasmon waveguide resonance Raman spectroscopy. Chapters 2-6 contain published manuscripts. This work develops a foundation of applied chemical measurements for numerous devices, including thin polymer films and photovoltaic devices.

Chapter 7 provides general conclusions to the preceding chapters as well as the future prospects of SA-Raman spectroscopy measurements. The appendix describes recent developments in SERS substrates with a primary focus on applications for single cell analysis. It is a section published in a review paper entitled "Single Cell Optical Imaging and Spectroscopy" in Chemical Reviews. 


\title{
CHAPTER 1: GENERAL INTRODUCTION
}

\begin{abstract}
Total internal reflection (TIR) Raman spectroscopy is a vibrational, non-destructive, surface sensitive technique for measuring chemical content of interfacial layers.

Scanning angle (SA) Raman spectroscopy is a variant of TIR-Raman spectroscopy where multiple incident angles are scanned while simultaneously collecting the Raman scatter. In this introduction chapter three SA-Raman spectroscopy configurations for chemical analysis including TIR-Raman spectroscopy, surface plasmon resonance (SPR) Raman spectroscopy and plasmon waveguide (PWR) Raman spectroscopy are reviewed. The general theory, experimental details, reflectivity and Raman scattering measurements behind each configuration are described. Recent applications and future trends for SARaman spectroscopy are explored with an emphasis on chemical analysis of thin polymer films.
\end{abstract}

\section{INTRODUCTION}

For chemical analyses of interfaces involving thin films it is important that the chemical content obtained from the bulk material be distinguished from that of the interface. ${ }^{1-2}$ Surface sensitive vibrational spectroscopies can be used to nondestructively probe interfacial content including detailed structural and orientation information. $^{3-4}$ A fundamental understanding of the interfacial structure is critical to determining proper function of numerous electronic devices and optical sensors. Common techniques used to measure thin materials at ambient laboratory conditions such as ellipsometry, atomic force microscopy, quartz crystal microbalance and optical 
interferometry offer surface sensitivity but do not directly provide information about chemical content. ${ }^{5}$ For this reason, surface sensitive vibrational spectroscopies are commonly used for interfacial analyses when chemical specificity is needed.

In order to measure chemical content near an interface, control over the axial spatial resolution perpendicular to the sample plane is needed. Confocal microscopy controls the axial resolution by restricting the collection of the emitted light to specific depths of the sample using a pinhole and focusing. In confocal microscopy the axial resolution is limited to the micron scale in the near infrared region, which limits its usefulness in applications requiring submicron surface sensitivity. Evanescent waves or standing-wave (waveguide) resonances can be used to control the amount of material probed near the interface. This is accomplished by using a total internal reflection (TIR) geometry. Many molecular spectroscopy techniques have incorporated this geometry including TIR-fluorescence, attenuated total reflectance infrared (ATR-IR) and TIRRaman spectroscopy for obtaining information near the surface.$^{6-7}$

Conventional Raman spectroscopy is not an inherently surface sensitive technique; however the TIR-Raman spectroscopy sampling geometry provides a means to generate a non-propagating wave near the interface. ${ }^{8}$ TIR-Raman spectroscopy has been previously used to measure polymer films, surfactants, plant material and adsorbates at various interfaces. ${ }^{9-12}$ In traditional TIR-Raman experiments, the illuminating laser beam is directed onto a prism/sample interface at an incident angle that is greater than the critical angle. The Raman scatter generated from the sample is confined to the interface as a result of the generated evanescent wave's limited penetration depth into the sample. 
The TIR-Raman geometry provides enhancements in the interfacial mean square electric field and can be applied to study a diverse range of samples without some of the limitations associated with resonance Raman spectroscopy or surface enhanced Raman spectroscopy.

ATR-IR spectroscopy is an analogue spectroscopy of TIR-Raman spectroscopy that measures the absorption of infrared radiation within an evanescent wave to give a near-surface composition of the material. In comparison to ATR-IR spectroscopy, TIRRaman spectroscopy provides the advantage of greater surface sensitivity because the penetration depth is proportional to the incident wavelength of light, which is commonly visible or near-IR light for Raman experiments and longer wavelengths are used for ATR-IR experiments. ${ }^{3}$ Therefore, TIR-Raman spectroscopy measurements provide smaller penetration depths into the sample. Additional advantages of TIR-Raman spectroscopy over ATR-IR spectroscopy are that the spectra can be easier to interpret because the penetration depth does not vary across the spectrum due to the monochromatic excitation wavelength. Finally, focusing a coherent, visible/near-IR laser beam is easier than a non-coherent mid-IR source.

In contrast to fixed angle TIR-Raman spectroscopy, scanning angle (SA) Raman spectroscopy is a technique that involves measuring light reflectivity at a variety of incident angles while simultaneously collecting Raman scatter at each angle. SA-Raman spectroscopy provides the flexibility of employing a variety of interface configurations when collecting Raman scatter over multiple incident angles. Implementing various configurations can be effectively utilized to combine Raman spectroscopy with other 
spectroscopic techniques such as surface plasmon resonance (SPR) and plasmon waveguide resonance (PWR). This introduction primary focuses on our research group's recent work in TIR-, SPR- and PWR-Raman spectroscopy and explores recent applications investigated with SA-Raman spectroscopy including measuring monolayers on gold films, submicron thin polymer films and fabricating waveguides for Raman signal enhancements.

Figure 1 represents the calculated reflectivity from the interface and the mean square electric field relative to the incident field summed from the interface for a sapphire/air (TIR) interface (left column), $50 \mathrm{~nm} \mathrm{Au/air} \mathrm{(SPR)} \mathrm{interface} \mathrm{(middle} \mathrm{column),}$ and $50 \mathrm{~nm} \mathrm{Au} / 630 \mathrm{~nm} \mathrm{SiO}$ 2 plasmon waveguide/air (PWR) interface (right column) for p- (yellow) and s- (cardinal red) polarized incident light. The benefits of using a thin gold film in the SPR or PWR configuration include: enhancing the Raman scatter, increasing the measurement reproducibility and reducing the prism background.

PWR-Raman spectroscopy employs non-radiative or "leaky" waveguides. Leaky waveguides produce an evanescent field which extends out of the waveguide interface. This evanescent wave is generated from imperfect confinement of light in the waveguide layer. The intensity depends on such relevant experimental parameters such as the polarization, refractive indices of the sample and the incident angle of light. These experimental parameters determine if the strongest portion on the electric field will occur in the waveguide or in the adjacent sample. Recently, PWR-Raman spectroscopy has taken advantage of the leaky waveguide phenomena to generate chemically specific information. ${ }^{13}$ PWR-Raman spectroscopy signal enhancements are comparable to those 
of SPR-Raman spectroscopy (Figure 1), but additional information from PWR-Raman can be obtained using both parallel and perpendicular electric fields in the waveguide/sample layer. An additional benefit of PWR-Raman spectroscopy not mentioned above includes alterative surface chemistries.

Recently, TIR-Raman spectroscopy has seen an increase in number of publications as modern technology has impacted the field of Raman spectroscopy. The number of TIR-Raman publications has increased 1.8x from 1990-1999 to 2000-2012. SA-Raman spectroscopy or scanning multiple incident angles has greatly contributed to this increase in publications. In publications from 2008-2012, 61\% of the TIR-Raman manuscripts involved scanning more than one incident angle (SA-Raman spectroscopy), while publications from $1980-2006$, only $20 \%$ or eight publications involved measuring more than one incident angle. The focal point of the chapter is on evaluating the SARaman spectroscopy interfacial configurations and is organized as follows; first, a brief introduction to the theory of Raman scattering, TIR-Raman spectroscopy, SPR-Raman spectroscopy and PWR-Raman spectroscopy is presented. This is essential for understanding the importance of implementing the various configurations. Next experimental considerations, reflectivity properties and TIR-Raman spectroscopy measurements at a prism interface will be discussed followed by similar sections dedicated to covering SPR-Raman spectroscopy and PWR-Raman spectroscopy. The last section of the chapter will discuss applications and future trends in SA-Raman spectroscopy. 


\section{THEORY}

Raman spectroscopy measures the inelastic scattering of a photon from a molecule. From the quantum mechanical point of view, Raman scattering occurs when a monochromatic source with frequency $\mathrm{v}_{0}$ encounters a molecule and then the scattered photon is Raman shifted to a lower stokes shift $\left(\mathrm{v}_{0^{-}} \mathrm{v}_{\mathrm{s}}\right)$ or a higher anti-stokes shift $\left(\mathrm{v}_{0}+\right.$ $\mathrm{v}_{\mathrm{s}}$ ) from that of the incident photon. The difference in energy between the incident photon and the Raman scattered photon is equal to the energy of a vibration of the scattering molecule. Scattering that occurs without a change of frequency is known as Rayleigh scattering. Spectroscopic transitions associated with Rayleigh scattering and Raman scattering are illustrated in Figure 2. Anti-stokes Raman scattering is less intense than stokes Raman scattering because anti-stokes Raman scattering occurs from an excited vibrational level within the electronic ground state. As governed by the Boltzmann distribution, the excited state population is lower than the ground state population at room temperature. In general, the probability of Raman scattering is low with approximately one in $10^{6}$ incident photons undergoing the process. For a vibrational mode to be Raman active there must be a change in the polarizability of the molecule, which is in contrast to the complimentary vibrational spectroscopy technique of IRabsorption spectroscopy where there must be a change in the molecular dipole moment. A change in polarizability can be described as a perturbation of the electron cloud with respect to the vibrational mode. A linear molecule containing $N$ atomic nuclei is predicted to have $3 N-5$ vibrational modes and a non-linear molecule $3 N-6$ vibrational modes. The cross section of Raman scattering is lower $\left(10^{-29-31} \mathrm{~cm}^{2} / \mathrm{sr}\right)$ compared to that of IR-absorption $\left(10^{-18-21} \mathrm{~cm}^{2} / \mathrm{sr}\right) .{ }^{14}$ Strong Raman scatters are molecules with distributed 
electron clouds such as a $\mathrm{C}=\mathrm{C}$ bond due to the easily disturbed $\pi$-electron cloud in an electric field. The general equation for the Raman scattering cross section for a stokes scattering process $\left(\mathrm{P}_{\mathrm{R}}\right)$ in units of photons/second is:

$$
P_{R}=P_{0} \sigma_{j} v_{0}\left(v_{0}-v_{s}\right)^{3} D d z
$$

where $\mathrm{P}_{0}$ is the incident photon flux, $\sigma$ is the Raman cross section with units of $\mathrm{cm}^{2}, \mathrm{D}$ is the number density of scatters (molecules per cubic centimeter), $\mathrm{dz}$ is the path length of the laser in the sample $(\mathrm{cm})$ and all frequency terms, $\left(v_{0}\right.$ and $\left.v_{s}\right)$, are expressed in relative wavenumber $\left(\mathrm{cm}^{-1}\right)$. A full derivation of this equation and a full quantum mechanical analysis to Raman scatter can be found elsewhere. ${ }^{15}$ It is important to mention that equation (0.1) highlights the importance of selecting a laser wavelength for Raman spectroscopy experiments due to the $v_{0}\left(v_{0}-v_{\mathrm{s}}\right)^{3}$ dependence of the Raman signal. ${ }^{16}$ Therefore, visible excitation will produce more Raman scatter than near-IR or mid-IR excitation. Competing phenomena such as fluorescence observed with UV and visible wavelengths can often mask the Raman spectrum. Many of the Raman spectrometers discussed in the chapter used excitation wavelengths between 633 and $785 \mathrm{~nm}$, which offers a good compromise between acceptable Raman scatter intensity and low fluorescence interference.

\section{TIR-Raman spectroscopy theory}

A diagram of a prism/sample interface, incident angle and collection optics for a scanning angle Raman spectroscopy setup is shown in Figure 3A. When the incident angle of light is below the critical angle, light will be transmitted though the sample and the Raman scatter generated is not surface sensitive. When the incident angle is above 
the critical angle, an evanescent wave will be generated at the prism/sample interface and exponentially decay into the sample. A hemispherical prism/800 nm polystyrene film/glycerol bulk solution in Figure 3B can be used to demonstrate the principle behind SA-Raman spectroscopy. The Raman spectrum collected at $54^{\circ}\left(\theta_{\mathrm{C}}=55.4^{\circ}\right)$ will be dominated by the bulk glycerol Raman signal with no easily detected polystyrene peaks as shown in Figure 3C. When collecting at an incident angle of $60^{\circ}$ under total internal reflection conditions the Raman spectra will represent that of only polystyrene and no bulk glycerol. This is due to the evanescent wave only penetrating the thin polystyrene film and not penetrating into the bulk glycerol solution. While an alternative approach to TIR-Raman spectroscopy is to send the light through a microscope objective off-axis using the "prismless" format, this approach has limitation when scanning the incident angle due to the narrow range of wavelengths that can be probed.

Under certain conditions the TIR sampling geometry provides a means to control the penetration depth in a sample to within a few microns of the interface. ${ }^{4}$ TIR occurs at an interface between two dielectrics with refractive indices $n_{1}$ and $n_{2}$, where $n_{1}>n_{2}$ with light travelling from the higher refractive index $\left(n_{1}\right)$ medium to the lower refractive index medium $\left(n_{2}\right)$ at an angle greater than the critical angle, $\theta_{c}$, as determined by Snell's law:

$$
\theta_{c}=\sin ^{-1} \frac{n_{2}}{n_{1}}
$$

Under TIR, there is no energy transmitted through the interface, but an evanescent wave that exponentially decays in the sample $\left(n_{2}\right)$ is generated. This is represented in equation (0.3) where the evanescent wave has an amplitude that decays exponentially with distance $z$ away from the interface. The depth of penetration of the evanescent wave 
or ' $d_{p}$ ' is expressed in equation (0.4). Since $d_{p}$ is dependent on the incident angle, the penetration depth of the evanescent wave can be controlled by varying the incident angle $(\theta)$ above the critical angle.

$$
\begin{aligned}
& E(z)=E_{0} e^{-z / d_{p}} \\
& d_{p}=\frac{\lambda}{2 \pi \sqrt{\sin ^{2} \theta_{1}-\frac{n_{1}}{n_{2}}}}
\end{aligned}
$$

In equation (0.4), $\lambda$ is defined as the wavelength of incident light and $\theta_{1}$ is the angle of incidence in the higher index medium. $d_{p}$ is the depth at which the evanescent wave intensity decreases to a value of 1/e. The depth over which Raman scatter is generated, $\mathrm{D}_{\mathrm{RS}}$, is $\mathrm{d}_{\mathrm{p}} / 2$ since Raman scatter is proportional to the intensity of light, thus decreasing twice as fast as the evanescent wave. For a given interface, $\mathrm{D}_{\mathrm{RS}}$ is at a maximum at the critical angle and decreases with increasing incident angle. The $\mathrm{D}_{\mathrm{RS}}$ can range from a few microns to hundreds of nanometers depending on the refractive indices of the interfacial species and wavelength of excitation. The $\mathrm{D}_{\mathrm{RS}}$ equation shows information from various depths in a sample can be obtained by controlling the incident angle of the excitation beam.

$$
D_{R S}=\frac{\lambda}{4 \pi}\left[\left(n_{\text {prism }}^{2} \sin ^{2} \theta\right)-n_{\text {sample }}{ }^{2}\right]^{-\frac{1}{2}}
$$

In equation (0.5), $\lambda$ is the wavelength of the incident light, $n_{1}$ is the index of refraction of the prism, $n_{2}$ is the index of refraction of the sample, and $\theta$ is the incident angle. A more in-depth review of the theory of Raman scattering from evanescent waves is presented elsewhere. $^{17}$ 
The intensity of the electric field at an interface can be calculated using Fresnel factors. ${ }^{18}$ These factors $\left(T_{x}, T_{y}\right.$ and $\left.T_{z}\right)$ describe the enhancement of the electric field at the prism/sample interface where $\mathrm{xy}$ is the sample plane and $\mathrm{z}$ extends perpendicularly from the interface. Parallel (p) polarized incident light will give rise to an electric field in the $\mathrm{x}$ and $\mathrm{z}$ directions $(0.6$ and 0.7$)$ and perpendicularly (s) polarized light will give rise to an electric field in the y plane (0.8). The Fresnel factors can be calculated as:

$$
\begin{gathered}
T_{x}=\frac{2 \cos \theta_{i}\left(\sin ^{2} \theta_{i}-n^{2}\right)+2 i n^{2} \cos ^{2} \theta_{i} \sqrt{\sin ^{2} \theta_{i}-n^{2}}}{n^{4} \cos ^{2} \theta_{i}+\sin ^{2} \theta_{i}-n^{2}} \\
T_{z}=\frac{2 n^{2} \cos ^{2} \theta_{i} \sin \theta_{i}-2 i \cos \theta_{i} \sin \theta_{i} \sqrt{\sin ^{2} \theta_{i}-n^{2}}}{n^{4} \cos ^{2} \theta_{i}+\sin ^{2} \theta_{i}-n^{2}} \\
T_{y}=\frac{2 \cos \theta_{i}-2 i \cos \theta_{i} \sqrt{\sin ^{2} \theta_{i}-n^{2}}}{1-n^{2}}
\end{gathered}
$$

where $\theta_{\mathrm{i}}$ is the incident angle and $n=n_{2} / n_{1}$. The final observation of solving the transmission coefficients or Fresnel factors is that the electric field is increased at the prism/sample interface under total internal reflection compared to normal illumination. The Fresnel factors $T_{y}$ and $T_{z}$ are often maximized at the critical angle depending on the system's configuration. Excitation with different polarizations can provide molecular orientation information as well as anisotropic refractive indices since the generated evanescent wave consists of perpendicular and parallel polarizations.

Incident laser light reflected at an interface between two transparent materials can provide valuable information in a TIR-Raman spectroscopy experiment. The reflectivity plot can determine the critical angle for a given interface and is marked by a sharp 
increase in the reflected light intensity. The reflection coefficients for s- and p- polarized incident light, $\mathrm{R}_{\mathrm{s}}$ and $\mathrm{R}_{\mathrm{p}}$, respectively, can be calculated using Fresnel equations as:

$$
\begin{aligned}
& R_{S}=\left|\frac{n_{1} \cos \theta_{i}-n_{2} \sqrt{1-\left(\frac{n_{1}}{n_{2}} \sin \theta_{i}\right)^{2}}}{n_{1} \cos \theta_{i}+n_{2} \sqrt{1-\left(\frac{n_{1}}{n_{2}} \sin \theta_{i}\right)^{2}}}\right|^{2} \\
& R_{p}=\left|\frac{n_{1} \sqrt{1-\left(\frac{n_{1}}{n_{2}} \sin \theta_{i}\right)^{2}}-n_{2} \cos \theta_{i}}{n_{1} \sqrt{1-\left(\frac{n_{1}}{n_{2}} \sin \theta_{i}\right)^{2}}+n_{2} \cos \theta_{i}}\right|^{2}
\end{aligned}
$$

The reflectivity obtained can determine the refractive indices of interfacial species for a specific incident wavelength and the critical angle at a given interface, all of which are important parameters for modeling the Raman scatter generated at an interface.

\section{SPR-Raman spectroscopy theory}

SA-Raman spectroscopy experiments using near-infrared excitation wavelengths can benefit from signal enhancement techniques when probing molecules with low Raman cross sections or small sample volumes. One technique to generate electric field enhancements is to implement smooth noble metal films at the interface, such as those used in SPR spectroscopy where the interfacial electric fields are amplified through the excitation of surface plasmons. Surface plasmons are charged density oscillations confined to the metal-dielectric interface under the appropriate excitation conditions. Surface plasmons are associated with the surface of metals and have significantly lower frequency (and energy) than bulk plasmons. The TIR geometry provides a convenient method of coupling light waves to surface plasmons. 
Surface plasmons can have a range of energies that depend on the complex dielectric function of the metal $\left(\varepsilon_{\mathrm{m}}\right)$ and the dielectric function of the adjacent medium $\left(\varepsilon_{\mathrm{d}}\right)$, as shown by the equation:

$$
k_{s p}=\frac{\omega}{c} \sqrt{\frac{\varepsilon_{m} \varepsilon_{d}}{\varepsilon_{m}+\varepsilon_{d}}}
$$

Where $\mathrm{k}_{\mathrm{sp}}$ is the wave vector magnitude of the surface plasmon, $\omega / \mathrm{c}$ is the wave vector in a vacuum, and the dielectric constant is the square of the index of refraction. One condition for the generation of surface plasmons is that $\varepsilon_{\mathrm{m}}$ and $\varepsilon_{\mathrm{d}}$ are of opposite signs (i.e. gold has a negative dielectric function in the visible and near-IR region, whereas water has a positive dielectric function). In the visible and near-IR region of the spectrum; gold, silver, copper, aluminum and platinum have negative real components $\varepsilon_{\mathrm{m}}$ $<0$ and can support surface plasmons with most common dielectrics $\varepsilon_{\mathrm{d}}>0$. With a proper metal/dielectric interface, surface plasmons can be excited by an evanescent wave in the Kretschmann geometry by matching the parallel component of the incident light's wave vector to the propagation constant of the surface plasmon. The condition of the parallel wave vector of the evanescent wave, $k_{\text {evan }}$ is expressed as:

$$
k_{\text {evan }}=\frac{2 \pi}{\lambda} n_{p} \sin \theta
$$

$\lambda$ is the excitation wavelength, $\mathrm{n}_{\mathrm{p}}$ is the refractive index of the prism, and $\theta$ is the incident angle. The electric field generated under these conditions is highest in intensity at the metal surface and decreases exponentially with increasing distance into the adjacent sample. The technique offers a relatively short penetration depth, with 1/e penetration depths on the order of $200 \mathrm{~nm}$ and can be calculated as: 


$$
d_{s p}=\frac{\lambda}{2 \pi}\left(\frac{\varepsilon_{m}+\varepsilon_{d}}{\varepsilon_{d}^{2}}\right)^{\frac{1}{2}}
$$

The polarization of the evanescent electric field generated by surface plasmons is unique with the main component oscillating in the $\mathrm{z}$ direction with a minor contribution in the $\mathrm{x}$ direction. Since s-polarized incident light cannot excite surface plasmons, p- polarized incident light is the only option for excitation of Raman scatter from analytes at the noble metal surface under TIR conditions. This is in contrast to the case without the metal film where both s- and p- polarizations can be used (TIR-Raman spectroscopy). The calculated MSEF enhancement at a gold interface is on the order of $10^{1}$ to $10^{2}$ at the SPR angle compared to non-TIR illumination Raman measurements. For example, a $50 \mathrm{~nm}$ Au film, aqueous analyte and $785 \mathrm{~nm}$ excitation produces a $25 \mathrm{x}$ enhancement in the MSEF compared to an interface without the gold film. The predicted MSEF enhancement of near-IR excitation is predicted to be $2 \mathrm{x}$ larger than visible excitation due to gold's smaller real and larger imaginary refractive index components in the near-IR part of the electromagnetic spectrum.

PWR and leaky-waveguide Raman spectroscopy theory

Leaky waveguides can be used to increase the interfacial electric field in comparison to the TIR configuration. The general theory of optical waveguides in thin films has been developed and presented in other work. ${ }^{19}$ Briefly, if the film of interest is acting as a waveguide, optical confinement requires the film to be of sufficient thickness, typically above a few hundred nanometers using near-IR light. The light is confined within the film acting as a waveguide. The portion of the guided light confided in the film and the portion that travels though a region extending outside and into the sample 
adjacent to the waveguide can be use to generate Raman scatter. Figure 4 shows a calculated mean square electric field distribution for a sapphire/polystyrene/air interface using different polystyrene thicknesses of (A) 300, (B) 500, (C) 600 and (D) $800 \mathrm{~nm}$. The mean square electric field generated within the polymer film spans between the vertical lines at the Au/PS interface to the vertical line at the polystyrene air interface.

In most common waveguide Raman spectroscopy experiments, a waveguide consisting of a dielectric layer, with refractive index $\mathrm{n}_{2}$, sandwiched between a prism of refractive index $\mathrm{n}_{1}$ and a sample of refractive index $\mathrm{n}_{3}$, such that $n_{1}>n_{2}>n_{3} .{ }^{20}$ The electric field in the dielectric layer is assumed to be sinusoidal while electric fields in the adjacent sample is assumed to have a decaying exponential form and can be calculated as follows:

$$
\begin{array}{cc}
E_{\text {prism }}=A_{\text {prism }} \exp \left(k_{z 1} z\right)(0.14 \mathrm{a}) & \mathrm{z}=0 \\
E_{\text {dielectric }}=A_{\text {dielectric }} \cos \left(k_{z 2} \theta\right) \quad(0.14 \mathrm{~b}) & \mathrm{z}=\mathrm{d} \\
E_{\text {sample }}=A_{\text {sample }} \exp \left[-k_{z 3}(z-d)\right](0.14 \mathrm{c}) &
\end{array}
$$

where $\mathrm{z}$ extends perpendicularly from the interface. When both the electric and magnetic fields at the prism/dielectric and dielectric/sample interfaces are matched, an eigenvalue condition can be written that must be satisfied for the polymer film to act as a waveguide:

$$
\tan ^{-1} \beta_{12}+\tan ^{-1} \beta_{32}+m \pi=k_{z 2} d
$$

where $\tan ^{-1} \beta_{12}$ and $\tan ^{-1} \beta_{32}$ are phase jumps that occur on reflection at the prism/waveguide and waveguide/sample interface respectively, while $k z_{2} d$ is the phase 
jump that occurs as the light transverses the sample of thickness $\mathrm{d}$ in the $\mathrm{z}$ direction. ${ }^{21}$ For this eigenvalue equation, the distribution of the electric field within the waveguide material can be calculated. ${ }^{20}$ The integer $m$ labels the mode and gives the number of antinodes in the electric field intensity distribution. Interference patterns are formed in the waveguide due to the large amount of total internal reflection that exists. Each of the TIR waves are summed to form an interference pattern and also produces a local evanescent field at the outer surface of the waveguide. Only a discrete number of incident angles are capable of allowing light to travel with minimum radiation loss along the waveguide.

Traditional waveguide Raman spectroscopy and plasmon resonance waveguide Raman spectroscopy differ in that PWR-Raman spectroscopy employs a thin metal film between the prism and dielectric material. PWR-Raman spectroscopy involves coupling surface plasmon resonance in a thin metal film with guided waves in an adjacent dielectric layer, resulting in excitation of two different resonances depending on incident light polarization. The coupling of the waveguide mode and a surface plasmon can occur when the propagation constant of the mode $\beta_{\mathrm{M}}$ is equal to the real part of the propagation constant of the surface plasmon $\beta_{\mathrm{sp}}$ :

$$
\beta_{\mathrm{M}}=\operatorname{Re}\left(\beta_{\mathrm{sp}}\right)
$$

The coupling condition in eq. (0.16) is satisfied only for a narrow range of incident angles. The reflected light from near or at the PWR angle is attenuated when the incident light resonates with guided modes. 


\section{TIR-RAMAN SPECTROSCOPY AT A PRISM INTERFACE}

Collecting Raman spectra at a prism interface by scanning incident angles above the critical angle will provide high axial resolution measurements perpendicular to the sample plane, where the evanescent wave produces Raman scatter generated from the sample. SA-Raman spectrometers can be custom built on an optical microscope platform with added automated variable angle optics to precisely and accurately control the angle of an incident laser beam on the prism/sample interface. The instrument angle range should be able to scan between $25-70^{\circ}$ to accompany a diverse number of interfaces including prism/sample/air, prism/aqueous sample and prism/organic sample. SA-Raman spectroscopy experiments can require an angle resolution of $0.05^{\circ}$ or finer because of the criticalness for modeling Raman data and reflectivity, which will be discussed further in the following sections. Our laboratory's SA-Raman spectrometer is integrated with a microscope where the Raman scatter is collected by a long working distance objective and then directed onto a spectrometer and detected with a charge-coupled device. ${ }^{22}$

Many fixed angle TIR-Raman spectroscopy experiments have been reported for interfacial chemical analysis. When collecting Raman scatter at a fixed angle the penetration depth is also fixed at a certain distance from the interface. The depth over which Raman scatter is collected from the interface can range from greater than one micron to less than a few hundred nanometers depending on the instrument configuration. SA-Raman spectroscopy has the ability for depth profiling measurements in the axial direction. Many of the SA-Raman spectroscopy experiments to date have measured 
homogeneous films and solutions to lay the foundation for measurements of heterogeneous systems. ${ }^{23}$

McKee and Smith reported collecting Raman scatter from a ZnSe/benzonitrile interface over a range of $180 \mathrm{~nm}$ to $35 \mathrm{~nm} .{ }^{22}$ Figure 5 shows the Raman peak area correlated with the $\mathrm{D}_{\mathrm{RS}}$. In order to optimize the experimental data to the calculated fit, the angle spread must be factored into the $D_{R S}$ equation. An incident angle spread is generated when the incoming beam is converging rather than collimated. The converging beam can produce a distribution of incident angles at each instrument setting as a result of focusing within the prism. When collecting Raman scatter close to the critical angle the angle spread can cause incident angles to be above and below the critical angle. This can cause issues for depth profiling measurements because angles below the critical angle will have contributions from scattering caused by light transmitted though the interface without surface sensitivity. Reducing the angle spread can minimize this issue.

Strategies on how to reduce the angle spread can be found in Reference $23 .{ }^{23}$ It should be noted that the angle spread will decrease with the incident angle and is therefore smaller at prism/air interfaces. Incident angles below the critical angle are not useful for depth profile measurements.

The $\mathrm{D}_{\mathrm{RS}}$ curve in Figure 5A does not account for the angle spread where Figure 5B factors in an angle spread of $\pm 1.45^{\circ}$. The calibration curve was validated by fitting the experimentally determined peak area to the $\mathrm{D}_{\mathrm{RS}}$ curve using a correlation factor that accounts for different Raman scattering cross sections for each Raman vibrational mode and the Raman instrument collection parameters. Ultimately, the achieved axial 
resolution perpendicular to the sample plane was approximately $34 \mathrm{~nm}$. Further preliminary depth profiling demonstrations measured a $56 \mu \mathrm{m} \pm 7 \mathrm{~nm}$ polystyrene film collected over an incident angle range of $40.3^{\circ}-66.6^{\circ}$, which corresponded to $\mathrm{D}_{\mathrm{RS}}$ values of $190 \mathrm{~nm}$ to $37 \mathrm{~nm}$. SA-Raman spectroscopy instrumentation equipped with near-IR excitation can also be applied to samples that exhibit fluorescence impurities, which allows for analysis of a wider range of industrial polymer films. ${ }^{24}$

Reflectivity spectra can predict the critical angle and incident angle where the maximum Raman scatter for a given interface is generated. The critical angle for a given interface is marked by a sharp increase in the reflected light intensity. For example, in Figure 2 the sapphire/air interface will produce a critical angle at $34.4^{\circ}$ (s-polarized) and $34.6^{\circ}$ (p-polarized). The critical angle of the sapphire/air interface for s- polarization is 0.2 degrees lower than for $\mathrm{p}$ - polarization due to the anisotropy of the sapphire prism $\left(n_{p-}\right.$ polarization $=1.764, n_{s-\text { polarization }}=1.753$ ). In depth profiling experiments, reflectivity can provide calibration parameters that enable modeling the $\mathrm{D}_{\mathrm{RS}}$ for depth profiling measurements. In addition, reflectivity data can also be used to experimentally determine the refractive indices. For example, Fresnel calculations were used to model the reflectivity data for a aqueous $1.25 \mathrm{M}$ pyridine solution and a neat nitrobenzene solution. ${ }^{25}$ The refractive indices were experimentally determined to be 1.344 and 1.537 , respectively. The reflectivity data can also be used to measure the instrument's polarization at the prism/sample interface. The polarization at the prism/sample interface can be measured by fitting the experimental reflectivity to calculated Fresnel equations. The ability to change the polarization from perpendicular to parallel polarization and vice 
versa can determine the molecular orientation and more efficiently collect Raman scatter depending on the efficiencies of the collection optics, gratings and detector.

The choice of prism material is critical for TIR-Raman experiments because the evanescent wave is only surface sensitive in the sample, not in the prism. The prism material will influence the penetration depth of the electric field, the intensity of the mean square electric field and the spectral background containing interference peaks. The spectral background and interfering Raman peaks in the $500-1800 \mathrm{~cm}^{-1}$ region have been evaluated from glass (SF-11), sapphire and ZnSe prisms. Sapphire prisms produce a Raman spectrum that is clear of interfering bands above $800 \mathrm{~cm}^{-1}$ but can also produce minimal background interference due to fluorescence impurities, and also allow optical coupling of a sample using index matching fluids. It has been found for sapphire and SF11 prisms that the use of a thin gold film can drastically reduce the Raman scattering background intensity by as much as $89-95 \%$ compared to without a gold film. ${ }^{25}$ The approach of using a thin gold film to reduce background signal from the prism material solves a significant problem in SA-Raman measurements.

The electric field generated under TIR conditions is increased at the prism/sample interface compared to normal illumination as predicated by Fresnel equations. The electric field increases at the interface with an increasing disparity between the prism and sample refractive indices. Samples with relatively low refractive indices such as water ( $n=1.33)$ offer $\sim 7 \mathrm{x}$ stronger electric fields and organic indices $(n=1.57) \sim 5 \mathrm{x}$ with $785 \mathrm{~nm}$ excitation at a sapphire interface. Therefore in SA-Raman spectroscopy experiments higher refractive index prisms are favorable if the prisms spectral background and Raman peaks are minimal. 


\section{SPR-RAMAN SPECTROSCOPY AT A GOLD INTERFACE}

SPR-Raman spectroscopy experiments are a modification of the TIR-Raman spectroscopy configuration with the addition of a thin, smooth, metal film between the prism and sample. Commonly, the prism or coupling substrate is coated with an adhesion layer such as titanium or chromium followed by 48-52 $\mathrm{nm}$ of a noble metal. Fresnel calculations predict $50 \mathrm{~nm}$ of gold as an optimal thickness for $785 \mathrm{~nm}$ excitation based on the generated electric field intensity. The average surface roughness of the gold film deposited by chemical vapor deposition is typically less than $1 \mathrm{~nm}$ as measured by atomic force microscopy. Fabricating metal films with minimal surface roughness is important for modeling reflectivity and to limit the surface enhancements that result from rough features, which complicate data analysis due to reproducibility issues.

Raman scattering from smooth metal films has historically been accomplished using silver films since the predicted electric field is 2-3 times higher than for gold films. However, silver films are much more likely to oxidize, therefore increasing the roughness of the metal surface that ultimately produces a decrease in the reflectivity and signal reproducibility for Raman spectroscopy measurements. In contrast to silver, gold films provide a chemically stable surface for generating surface plasmons, favorable optical properties in the visible/near-IR and are suitable for a broad range of samples.

The SPR angle is the angle with the greatest attenuation of the reflected light intensity and is associated with the excitation of surface plasmons, inducing surface plasmon resonance. The experimental reflectivity data can be modeled by Fresnel calculations with the appropriate angle spread as described above. Fresnel's calculations 
can predict the location of the critical angle and SPR angle. McKee et al. demonstrated this using a SA-Raman instrument to experimentally determine the SPR and critical angle to within $0.3^{\circ} .^{25}$ Near the SPR angle, the modeled Raman peak area is approximately the mirror image of the SPR curve to suggest the reflectivity data can predict the collected Raman signal.

The greatest Raman peak intensity for SPR-Raman measurements is not at the critical angle but rather the SPR angle. ${ }^{26}$ Figure 6 shows the calculated mean-square electric field (MSEF) for (6A) sapphire/gold/1.25M pyridine interface and (6B) sapphire/gold/nitrobenzene interface. ${ }^{25}$ The measured Raman spectra of $1.25 \mathrm{M}$ pyridine or nitrobenzene at a sapphire/gold/analyte interface are shown in Figure 6C and 6D, respectively. As predicted by the MSEF the measured Raman spectra show more Raman scatter will be generated at the gold/aqueous interface than the gold/organic interface. This conclusion assumes the same amount of Raman scatters are being probed. The calculated enhancements are $11 x$ for aqueous pyridine $(n=1.344)$ and $9 x$ for nitrobenzene $(n=1.472)$ at a gold film interface compared to a sapphire interface. ${ }^{25}$ The experimental enhancement factors ranged from $4.3 \mathrm{x}$ to $4.6 \mathrm{x}$ for pyridine and $2.2 \mathrm{x}$ to $3.7 \mathrm{x}$ for nitrobenzene depending on the individual vibrational mode. The discrepancy in enhancement between the gold and the sapphire interface could be from suboptimal gold films, small changes in collection efficiency at different excitation angles and fluctuations in the background signal as surface plasmons are excited within the gold.

PWR-RAMAN SPECTROSCOPY AT A DIELECTRIC INTERFACE 
PWR-Raman spectroscopy introduces chemical specificity to traditional PWR spectroscopy experiments, enhancements of the electric field in comparison to TIRRaman spectroscopy and the capability to generate a y- component to the interfacial electric field that is not possible in SPR experiments. In addition the dielectric layer can provide a protective coating over the metal surface. Metallic SPR substrates can be modified to produce PWR-Raman spectroscopy substrates with the addition of a transparent dielectric layer of a certain thickness. The dielectric layer can be deposited onto the metal by spin coating, free casting or vacuum deposition. Spin coating is a rapid technique that allows the control of the dielectric thickness, flatness and homogeneity. ${ }^{27}$ Plasma-enhanced vapor deposition (PECVD) and chemical vapor deposition can also be used to fabricate PWR-Raman spectroscopy substrates with more control over the thickness. Dielectric materials such as $\mathrm{SiO}_{2}, \mathrm{TiO}_{2}$ and $\mathrm{Si}_{3} \mathrm{~N}_{4}$ could potentially be used for PWR-Raman spectroscopy depending on the refractive index of the prism and dielectric thickness. Other dielectric materials are currently being explored for PWR-Raman spectroscopy measurements.

Reflectivity measurements near the PWR angle presents a sharp attenuation of the reflected light with both p- and s- polarization. The incident angle(s) where plasmon waveguide resonances are excited depend on the thickness of the dielectric layer (as well as other interface properties), thus unique reflectivity spectra are obtained as incident angles are scanned. Plasmon waveguides reduce the full width at half-maximum of the reflectivity curves and increase precision over traditional SPR spectroscopy. ${ }^{28}$ For example, the full width at half maximum of the reflectivity minima with p-polarized incident light decreases roughly 6x from an SPR angle to a PWR angle. Similar to SPR- 
Raman spectroscopy, reflectivity and Raman scattering intensities have been modeled with PWR-Raman spectroscopy. ${ }^{13}$

The predicted intensity of Raman scatter for PWR, SPR and TIR follows the order: SPR >PWR > TIR for p-polarization and PWR $>>$ TIR for s- polarization. Experimentally the intensity of the PWR-Raman peaks will be limited by the instrument's angle resolution. An incident angle uncertainty of $0.05^{\circ}$ can significantly alter the amount of Raman scatter that is generated at the PWR interface using s-polarization. The angle spread can also result in a decrease in the amount of Raman scatter collected, particularly in a Raman spectrum obtained at the PWR interface with s-polarized light.

Several advantages can arise from PWR-Raman measurements. The first is the well-defined electric field distribution from within the waveguide material, which can be exploited to give spatial information about the Raman scattering sample. ${ }^{29}$ When polarization can be altered, the orientation of molecules within orientated layers can be determined. Second, enhanced electric field intensities exist within the waveguide for generating Raman scatter, giving increased sensitivity for thin film materials. The Raman spectra of a sapphire prism/50 nm gold/polystyrene/air interface for polystyrene thicknesses of (A) $276 \mathrm{~nm}$, (B) $412 \mathrm{~nm}$, (C) $595 \mathrm{~nm}$ collected with p-polarization and (D) $595 \mathrm{~nm}$ collected with s-polarization can be seen in Figure 7.

The drawback of PWR-Raman spectroscopy is the restricted number of materials that can be used as waveguides and the thickness requirement. Ideally, these materials must have a minimum thickness of a few hundred nanometers to be able to support guided waves. An additional drawback is the Raman scattering generated from the 
waveguide material can interfere when the sample layer adjacent to the waveguide is of interest. For example, several hundred nanometers of material may contribute to the spectral background; this is due to the strong electric field intensity in the waveguide material. Recent data shows that using a $650 \mathrm{~nm}$ silica waveguide actually decreases the sapphire peak intensity of the $750 \mathrm{~cm}^{-1}$ peak by $11 \mathrm{x}$ and $58 \mathrm{x}$ for using $\mathrm{p}$ - or s- polarized light, respectively. ${ }^{13}$ Overall, there was no sign of an increased spectral background from the silica waveguide. To date there is no published data on reducing other prism backgrounds such as SF-11 or ZnSe with metal films or waveguides, although unpublished data show SF-11 prism background is reduced with the addition of a 50-nm Au layer.

\section{APPLICATIONS}

\section{SA-Raman applications: Analysis of thin polymer films}

Thin polymer films are critical components of many devices including optical sensors, coatings and medical equipment. ${ }^{30}$ SA-Raman spectroscopy can be used to control and model the electric field distribution in polymer films using incident angles above and below the critical angle. ${ }^{31}$ Extending experiments to include incident angles below the critical angle increases the amount of incident angles in the data set and allows beam penetration into the sample, which can lead to more Raman scattering than from an evanescent wave. Kivioja and co-workers analyzed polystyrene on polypropylene films at a fixed incident angle that was greater than the critical angle. ${ }^{32}$ Fontaine and Furtek measured polystyrene using the waveguide mode to identify a $1024 \mathrm{~nm}$ polystyrene

film. ${ }^{33}$ Recently, Meyer et al. measured polystyrene with thicknesses ranging from 1.8 
$\mu \mathrm{m}$ to $400 \mathrm{~nm}$ with SA-Raman spectroscopy as discussed in chapter $2 .^{34}$ The wide range of polystyrene thicknesses were determined by fitting the experimental Raman scatter generated at specific incident angles with integrated electric field distributions. A 2D plot of experimental Raman scatter collected from $58^{\circ}$ to $70^{\circ}$ for a $950-\mathrm{nm}$ polystyrene film can be seen in Figure 8. SA-Raman spectroscopy was carried out on polystyrene films ranging from $4-12 \%(\mathrm{w} / \mathrm{v})$. The experimental data was used in a calibration curve to determine the polymer film thickness. The average uncertainty in the SA-Raman determinations for all films was $4 \%$ and agreed closely with those obtained from optical interferometry. This proof of principle study is an example of how SA-Raman spectroscopy can be applied to measure chemical composition and thicknesses of interfacial polymer films.

Future applications of SA-Raman spectroscopy at a prism interface could include analysis of thin film gradient profiles, inter-diffusion and determining buried interface locations in multilayer polymer films. Studies involving semiconducting polymeric materials with SA-Raman spectroscopy could be used to gain information of molecular ordering as a function of depth and phase separation. ${ }^{35}$ In order to more accurately and precisely analyze these thin polymer films, the incident angle spread has to be reduced to more effectively model the calculated reflectivity and the $\mathrm{D}_{\mathrm{RS}}$ curve. The ability to nondestructively and precisely resolve the spatial information in thin polymer films can make SA-Raman spectroscopy a powerful analytical technique. In the future SA-Raman spectroscopy can be used as a balancing technique to confocal Raman where SA-Raman spectroscopy can study spatial information from polymer films with thicknesses less than 1-2 $\mu \mathrm{m}$ and confocal can study the thicker samples. 
SPR-Raman spectroscopy applications: Detection of monolayers

Recently, $633 \mathrm{~nm}$ and $647 \mathrm{~nm}$ excitation wavelengths were used to measure the resonant Raman scatter from Nile blue at a sapphire/gold/water interface and a sapphire/gold/air interface. ${ }^{36}$ Monolayer sensitivity for non-resonant analytes has recently been demonstrated for the first time using the SPR-Raman spectroscopy configuration. ${ }^{25}$ A monolayer of adsorbate was measured with SPR-Raman spectroscopy with $785 \mathrm{~nm}$ near-IR excitation. Benzenethiol and 4-mercaptopyridine were prepared on a gold film and measured at the SPR angle. The S/N ratios obtained from a one minute acquisition were 6.8 and 16.3 for benzenethiol and 4-mercaptopyridine, respectively. This was the first study to demonstrate SPR-Raman spectroscopy of monolayer sensitivity without resonant enhancement. SPR-Raman spectroscopy can also be used to study thin polymer films. The chemically specific information obtained from SPRRaman spectroscopy measurements has the potential to impact developing technologies involving a metal/polymer film commonly used for energy storage and electronic devices. Fabrication of SPR-Raman spectroscopy sensors have yet to fully explore bimetallic films. These films consist of $42-45 \mathrm{~nm}$ of silver with a thin coating of gold (3-5 $\mathrm{nm})$ to protect the silver from oxidizing and diminishing the reflectivity properties.

PWR-Raman spectroscopy applications: Analysis of thin polymer films

SA-Raman plasmon waveguide spectroscopy can be used to determine the thickness and chemical content of thin polymer films. The technique was recently used to gain chemical information while simultaneously measuring the polystyrene thicknesses on gold and is discussed in Chapter $3{ }^{28}$ The film thicknesses obtained from SA-Raman 
spectroscopy agreed with values obtained from optical interferometry with an average difference of 2.6\%. PWR-Raman spectroscopy's ability to determine the polymer thickness complements the reproducible and predictable Raman signal enhancements.

PWR-Raman spectroscopy can be used to measure sub-100 nm polymer films using a silica plasmon waveguide interface. McKee et al. obtained a Raman spectrum from a $100-n m$ polystyrene film at a sapphire/gold/silica interface. ${ }^{22}$ The Raman peak area for the $1001 \mathrm{~cm}^{-1}$ stretching mode is 1.9 and 4.1 times greater in the PWR spectra than in the TIR-Raman spectra for s- and p-polarized incident light, respectively. If the instrument's angle resolution was improved from $0.05^{\circ}$ to $0.01^{\circ}$ the $1001 \mathrm{~cm}^{-1}$ peak intensity to $9.5(\mathrm{~s})$ and $1.7(\mathrm{p})$ times. A monolayer of tri(methyl)phenylsilane was used to further demonstrate the increased sensitivity gained using PWR-Raman spectroscopy. This technique can potentially provide an undemanding method to elucidate molecular orientation of adsorbates and thin films, which may not be possible using SPR Raman spectroscopy. One direction of PWR-Raman spectroscopy that has yet to be fully explored is the fabrication of porous waveguide films for analyte immobilization inside the pores. This would fully take advantage of the high mean square electric field intensity that can be generated in the waveguide while increasing the analyte charge density. In addition to SA-Raman spectrometers being commercialized, further designs and concepts need to be developed to increase the surface sensitivity and general application of PWR-Raman spectroscopy before it becomes a standard tool used in Raman spectroscopy. 


\section{REFERENCES}

(1) Bohn, P. W. Annual Review of Materials Science 1997, 27, 469.

(2) Mirabella, F. M., Internal Reflection Spectroscopy; Marcel Dekker Inc. :

New York, New York, 1993; Vol. 15.

(3) Fina, L. J. Applied Spectroscopy Reviews 1994, 29, 309.

(4) Knoll, W. Makromolekulare Chemie-Macromolecular Chemistry and Physics 1991, 192, 2827.

(5) Novotny, L.; Stranick, S. J. Annu Rev Phys Chem 2006, 57, 303.

(6) Axelrod, D. Methods Cell Biol. 2008, 89, 169.

(7) Woods, D. A.; Bain, C. D. Analyst 2012, 137, 35.

(8) Ikeshoji, T.; Ono, Y.; Mizuno, T. Appl Opt 1973, 12, 2236.

(9) Iwamoto, R.; Ohta, K.; Miya, M.; Mima, S. Applied Spectroscopy 1981, 35,584 .

(10) Greene, P. R.; Bain, C. D. Colloids Surf B Biointerfaces 2005, 45, 174.

(11) Woods, D. A.; Petkov, J.; Bain, C. D. J Phys Chem B 2011, 115, 7353.

(12) Woods, D. A.; Petkov, J.; Bain, C. D. J Phys Chem B 2011, 115, 7341.

(13) McKee, K. J.; Meyer, M. W.; Smith, E. A. Analytical Chemistry 2012, 84, 9049.

(14) Pieczonka, N. P. W.; Aroca, R. F. Chemical Society Reviews 2008, 37, 946.

(15) Long, D. A., The Raman Effect: A unified Treatment of the Theory of Raman Scattering by Molecules; John Wiley \& Sons Ltd.: New York, NY, 2002, pp 611. 
(16) Raman Spectroscopy for Chemical Analysis; McCreery, R. L., Ed.; WileyInterscience, 2000; Vol. 157, pp 263.

(17) Dhooge, L.; Vigoureux, J. M. Chemical Physics Letters 1979, 65, 500.

(18) Corn, R. M.; http://corninfor.ps.uci.edu/calculations.html.

(19) Syms, R.; Cozens, J. Optical Guided Waves and Devices; McGraw-Hill Book Company: London, England, 1992.

(20) Zimba, C. G.; Hallmark, V. M.; Turrell, S.; Swalen, J. D.; Rabolt, J. F. Journal of Physical Chemistry 1990, 94, 939.

(21) Khomchenko, A. V. Thin Films and Nanostructures; Elsevier: San Diego, CA, 2005; Vol. 33.

(22) McKee, K. J.; Smith, E. A. Review of Scientific Instruments 2010, 81, 043106.

(23) Fontaine, N. H.; Furtak, T. E. Journal of the Optical Society of America BOptical Physics 1997, 14, 3342.

(24) Ishizaki, F.; Kim, M. Japanese Journal of Applied Physics 2008, 47, 1621.

(25) McKee, K. J.; Meyer, M. W.; Smith, E. A. Analytical Chemistry 2012, 84, 4300.

(26) Siirila, A. R.; Bohn, P. W. Langmuir 1991, 7, 2188.

(27) Hall, D. B.; Underhill, P.; Torkelson, J. M. Polymer Engineering and Science 1998, 38, 2039.

(28) Meyer, M. W., McKee, K.J., Nguyen, H.T., Smith, E.A. J. Phys. Chem. C 2012, 47, 24987-24992. 
(29) Pemberton, J. E., Characterization of Organic Thin Films; ButterworthHeinemann: Stonehamn, MA, 1995, 87.

(30) Ebata, Y.; Croll, A. B.; Crosby, A. J. Soft Matter 2012, 8, 9086-9091.

(31) Meyer, M. W.; Smith, E. A. MRS Online Proc. Lib. 2013, 1552, DOI:10.1557/opl.2013.133.

(32) Kivioja, A. O.; Jaaskelainen, A. S.; Ahtee, V.; Vuorinen, T. Vibrational Spectroscopy 2012, 61, 1.

(33) Fontaine, N. H.; Furtak, T. E. Physical Review B 1998, 57, 3807.

(34) Meyer, M. W.; Nguyen, V. H. T.; Smith, E. A. Vibrational Spectroscopy 2013, 65, 94.

(35) Tsoi, W. C.; James, D. T.; Kim, J. S.; Nicholson, P. G.; Murphy, C. E.; Bradley, D. D. C.; Nelson, J.; Kim, J. S. Journal of the American Chemical Society 2011, 133, 9834.

(36) Meyer, S. A.; Le Ru, E. C.; Etchegoin, P. G. Analytical Chemistry 2011, 83, 2337. 


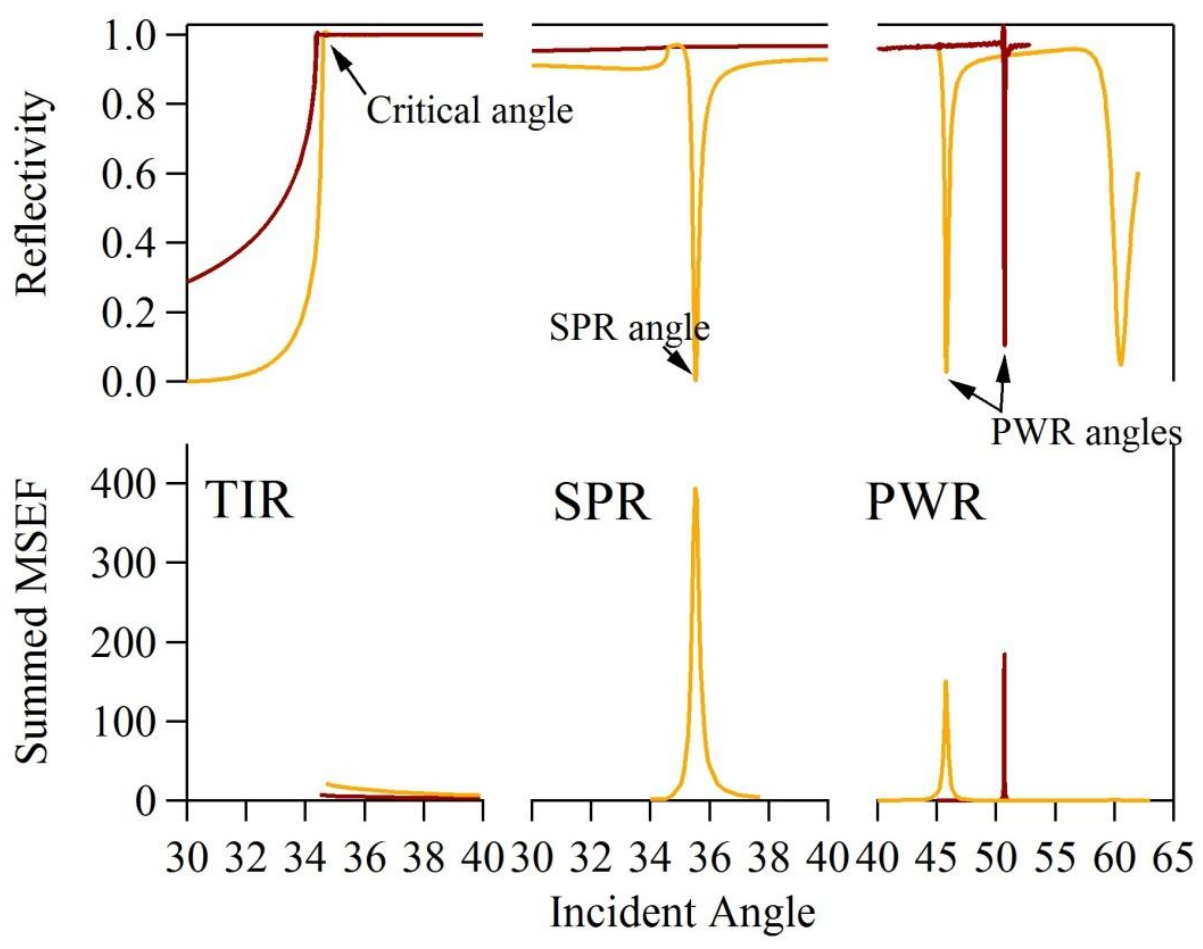

Figure 1. Calculated reflectivity from the TIR, SPR and PWR interfaces (top row); summed mean square electric field relative to the incident field (bottom row). TIR at a sapphire/air interface (left), SPR at a sapphire/gold/air interface (middle) and PWR at a sapphire/gold/silica/air interface (right). Excitation with s- polarization (cardinal red) and p- polarization (yellow). 


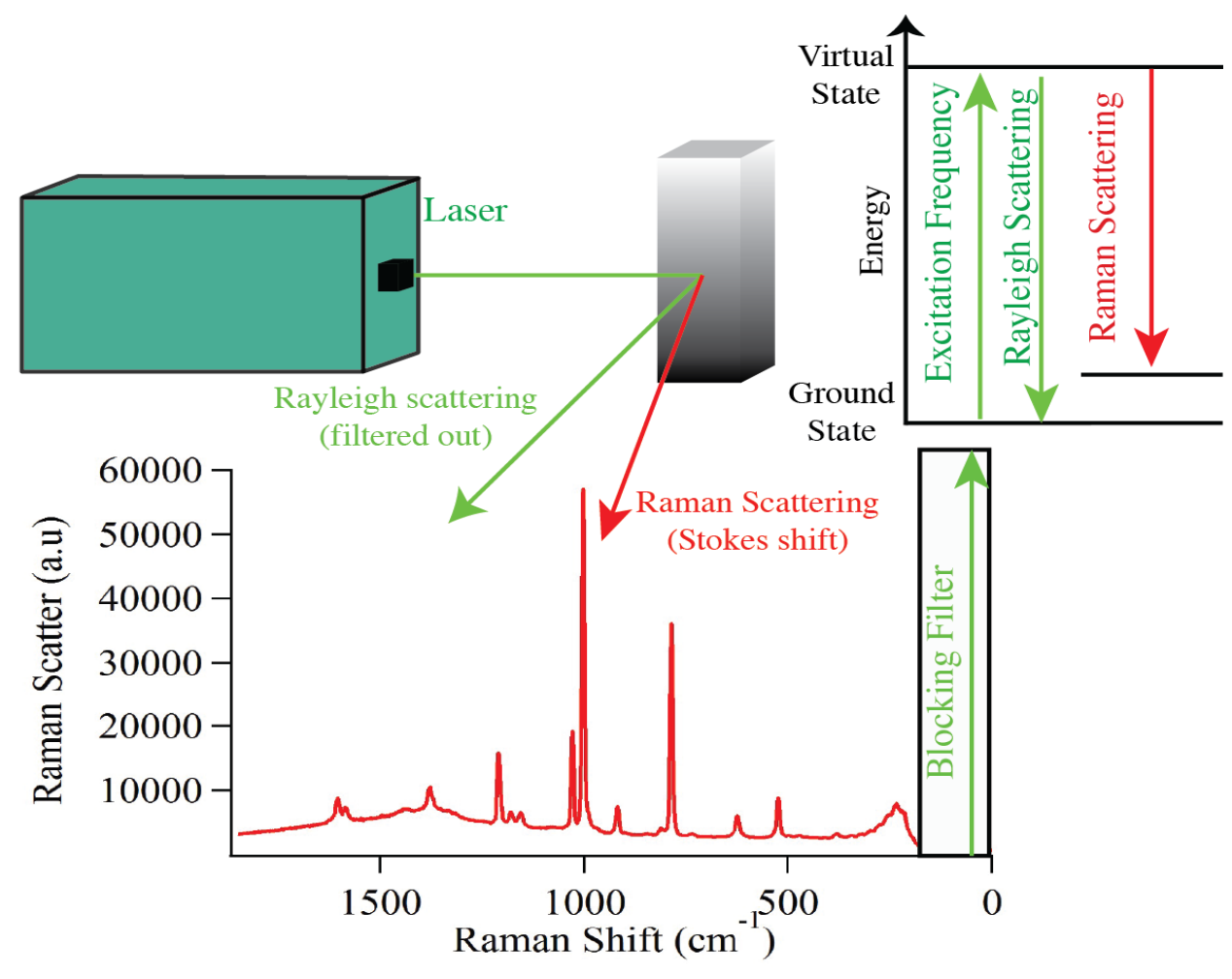

Figure 2. Spectroscopic transitions associated with Rayleigh scattering and Raman scattering. Raman scattering occurs when a monochromatic source encounters a molecule and then the scattered photon is Raman shifted to a lower stokes shift or a higher anti-stokes shift (not shown) from that of the incident photon. 


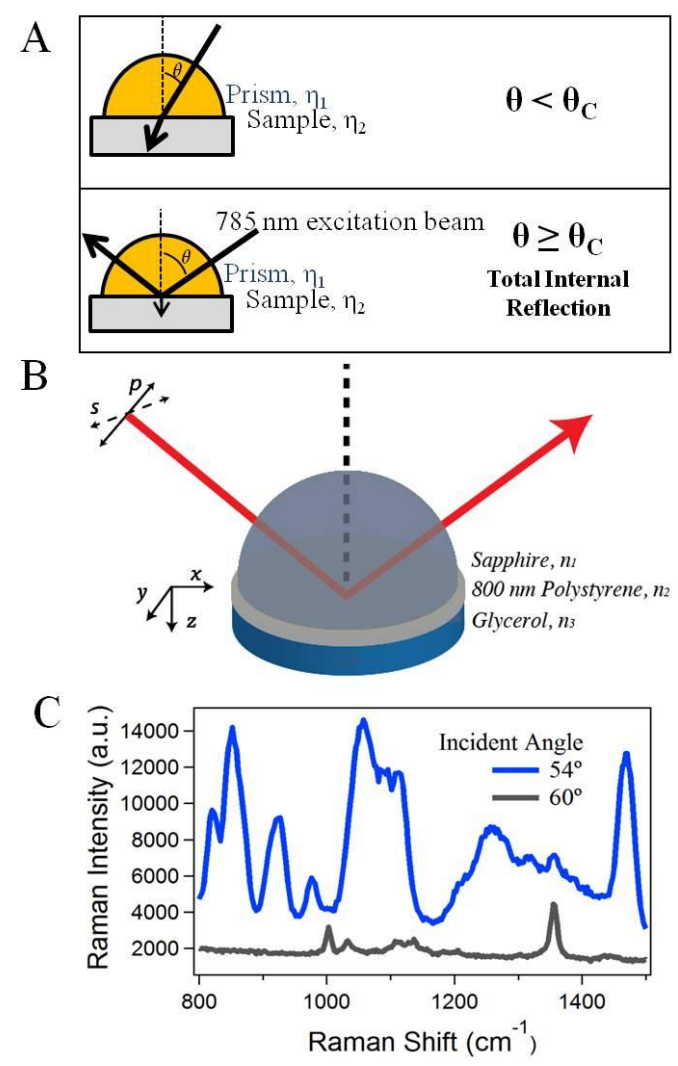

Figure 3. Diagram of a scanning angle Raman spectroscopy configuration. (3A) The incident light is transmitted though the sample when the incident angle is below the critical angle (bottom) and incident light is confined to the surface by generating an evanescent wave when the incident angle is above the critical angle (top). (3B) Example of a sapphire prism/800 nm polystyrene film and a bulk glycerol solution. (3C) Raman spectra of the materials in Figure $3 \mathrm{~B}$ at $54^{\circ}$ (blue) and $60^{\circ}$ (grey). The critical angle for the stack is $55.4^{\circ}$. 


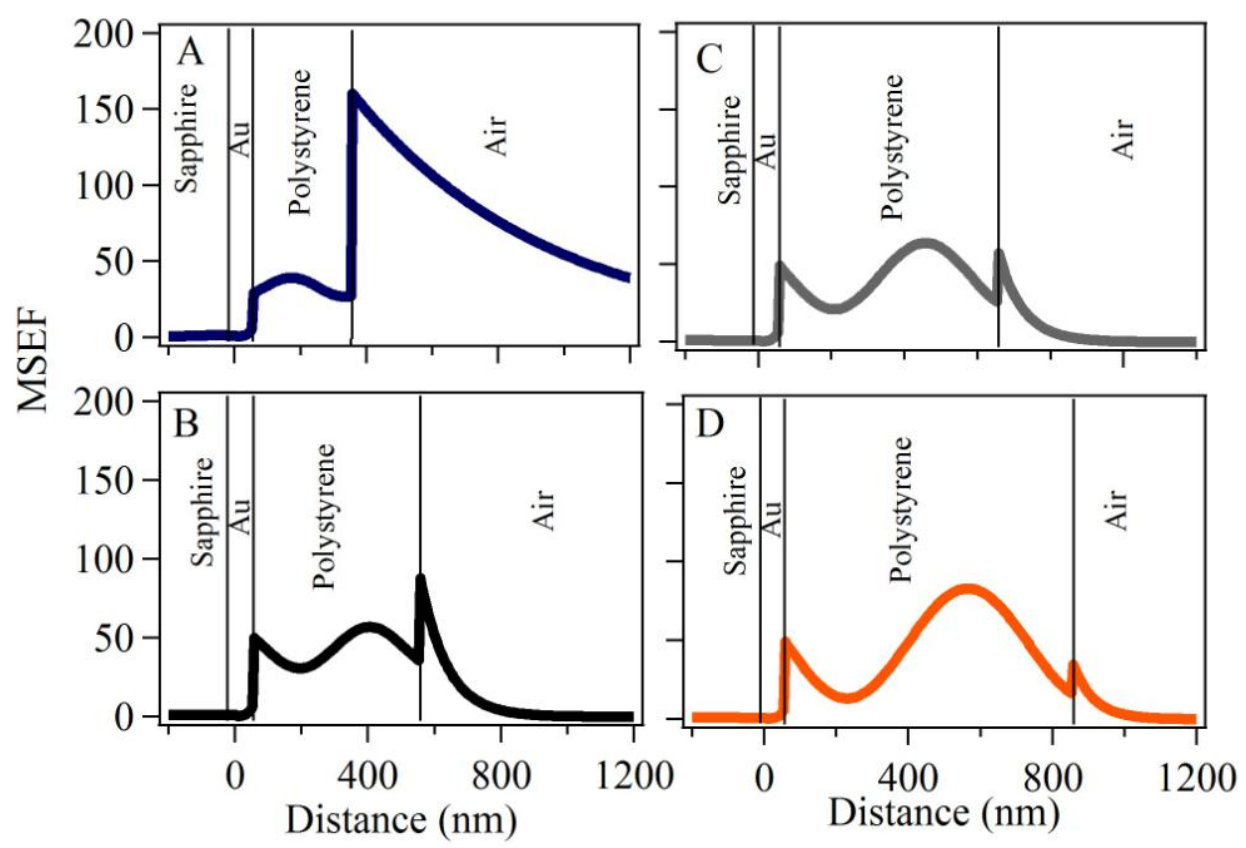

Figure 4. Calculated MSEF distribution of a sapphire/gold/polystyrene/air interface with polystyrene thicknesses of (4A) $300 \mathrm{~nm}$ (blue), (4B) $500 \mathrm{~nm}$ (black), (4C) $600 \mathrm{~nm}$ (grey), and (4D) $800 \mathrm{~nm}$ (orange). The electric field spans between the vertical lines at the gold/polystyrene interface to the vertical line at the polystyrene/air interface. 


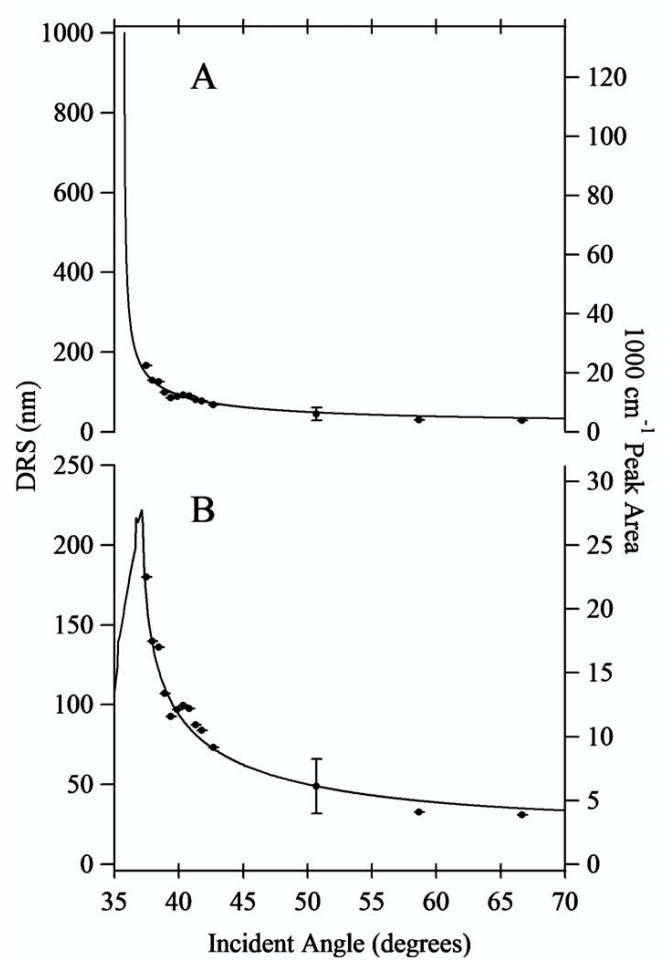

Figure 5. Raman peak areas (circles) for the $1000 \mathrm{~cm}^{-1}$ benzonitrile peak and $\mathrm{D}_{\mathrm{RS}}$ curves (line) with (5A) no angle spread and (5B) $1.45^{\circ}$ angle spread above and below the indicated $\mathrm{x}$-axis value. The error bars represent the average uncertainty in peak areas measured for all incident angles. Reprinted with permission from reference 22 . Copyright 2010 Review of Scientific Instruments. 

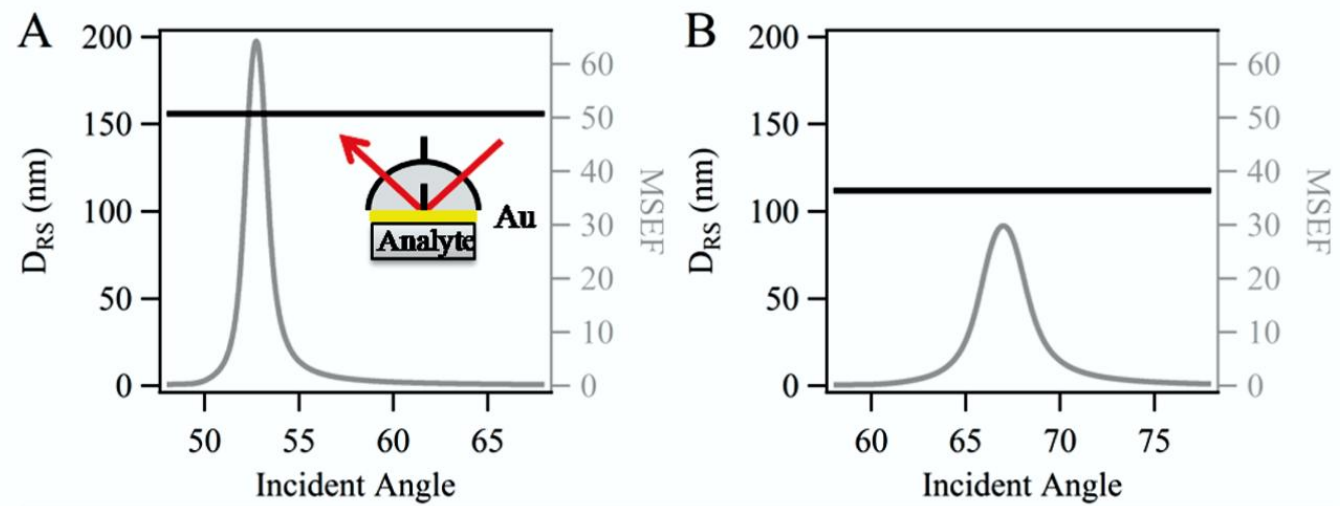

C

D
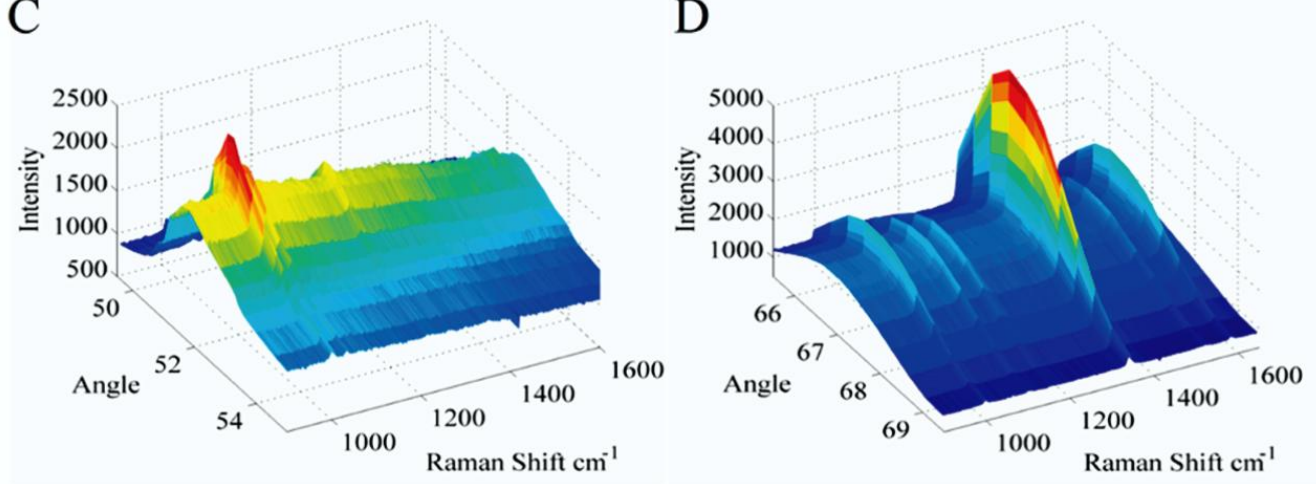

Figure 6. Calculated MSEF (grey) and $\mathrm{D}_{\mathrm{RS}}$ (black) for a (6A) sapphire/gold/1.25M pyridine interface or (6B) sapphire/gold/nitrobenzene interface with a (A) $\pm 0.25^{\circ}$ or (B) \pm $0.50^{\circ}$ incident angle spread. The measured Raman spectra of (6C) $1.25 \mathrm{M}$ pyridine or (6D) nitrobenzene at a sapphire/gold/analyte interface are shown from $50-55^{\circ}$ and $65-$ $70^{\circ}$, respectively. Reproduced with permission from reference 13. Copyright 2012 American Chemical Society. 


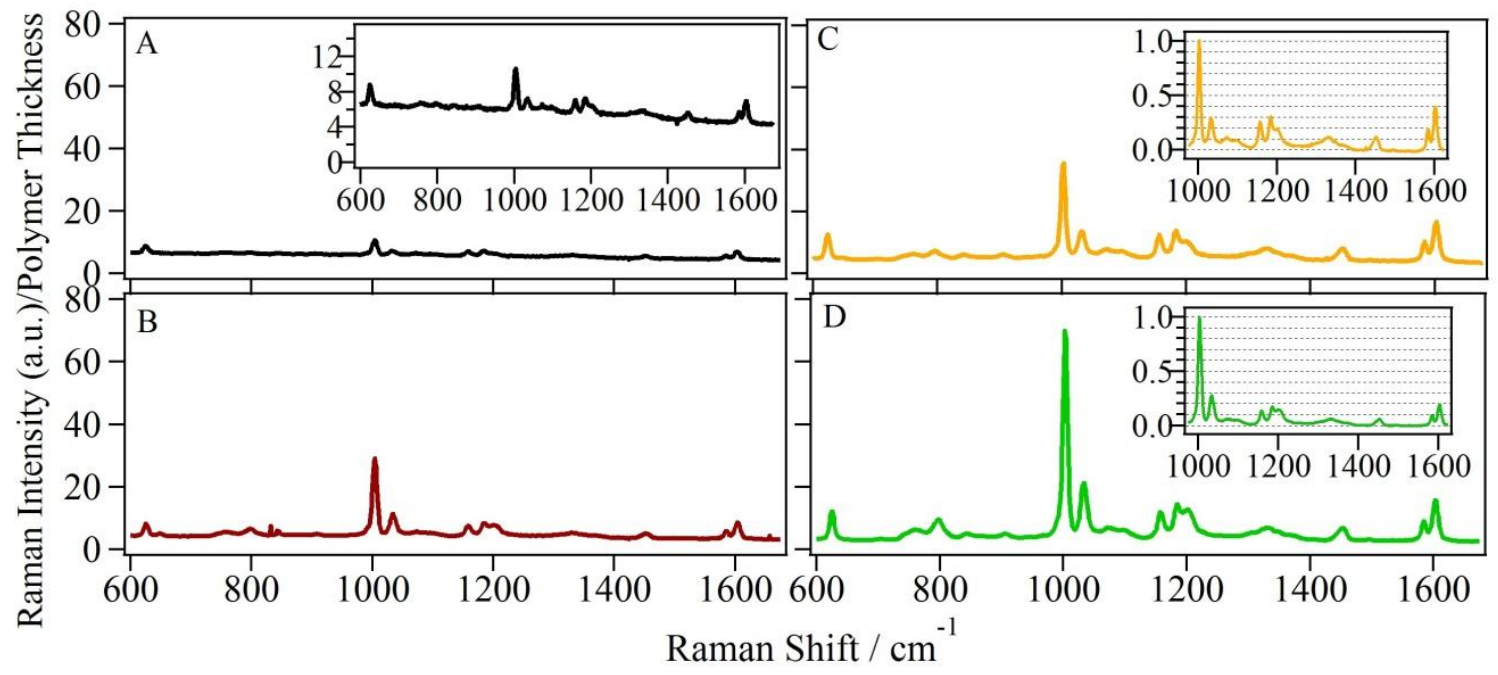

Figure 7. Raman spectra of a sapphire prism/50 nm gold/polystyrene (PS)/air interface: (7A) $276 \mathrm{~nm}$ PS; (7B) $412 \mathrm{~nm}$ PS; (7C) $595 \mathrm{~nm}$ PS collected with p-polarization incident excitation at $68.40^{\circ}, 40.52^{\circ}$ and $50.77^{\circ}$, respectively. (7D) Sapphire prism/50 nm gold/595 $\mathrm{nm}$ polystyrene (PS)/air interface collected with s-polarized incident excitation at $41.80^{\circ}$. All spectra have been divided by their respective film thicknesses to emphasize differences in the scattering intensity due to differences in the MSEF. Reproduced with permission from reference 27. Copyright 2013 American Chemical Society. 


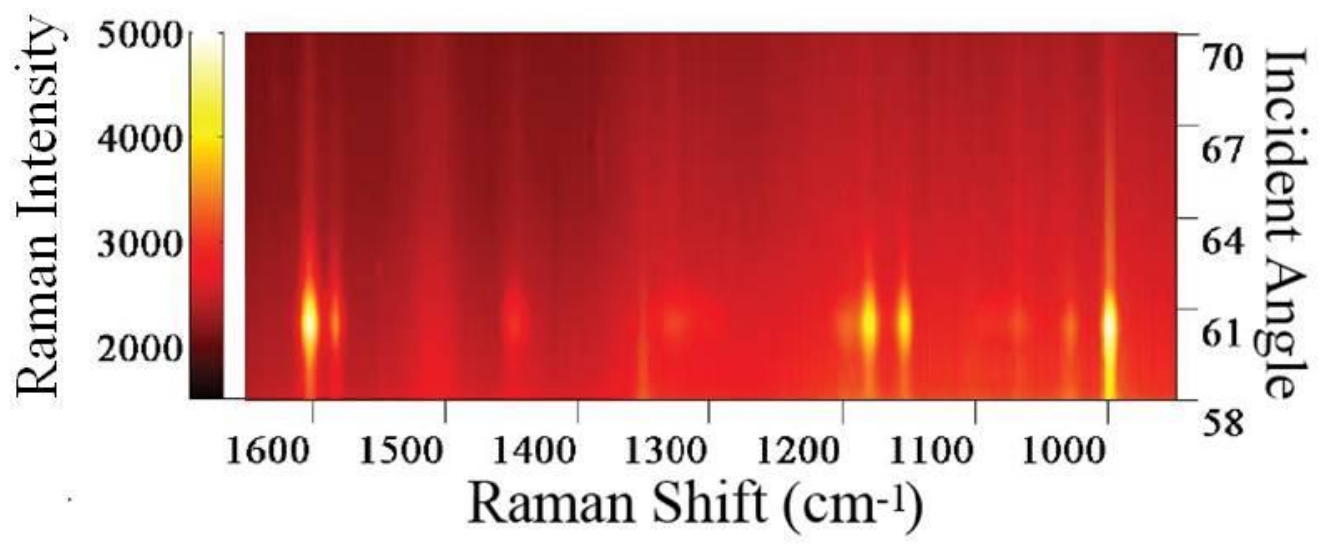

Figure 8. Plot of experimental Raman scatter collected from $58^{\circ}$ to $70^{\circ}$ for a $950 \mathrm{~nm}$ polystyrene film. The data was collected with an excitation power of $210 \mathrm{~mW}$ at the prism and the acquisition time was $60 \mathrm{~s}$ per spectrum. 


\title{
CHAPTER 2: SCANNING ANGLE RAMAN SPECTROSCOPY MEASUREMENTS OF THIN \\ POLYMER FILMS FOR THICKNESS AND COMPOSITION ANALYSES
}

A paper published in J.of Vib. Spec., 2012, 65, 94-100.

Matthew W. Meyer, Vy H.T. Nguyen and Emily A. Smith

Ames Laboratory, U. S. Department of Energy, Ames, Iowa 50011-3111, and

Department of Chemistry, Iowa State University, Ames, Iowa 50011-3111

\begin{abstract}
Scanning angle (SA) Raman spectroscopy was used to measure the thickness and composition of polystyrene films. A sapphire prism was optically coupled to a sapphire substrate on which 6 to $12 \% \mathrm{w} / \mathrm{v}$ polystyrene in toluene was spin coated. Raman spectra were collected as the incident angle of the p-polarized, 785-nm excitation laser was varied from 56 to 70 degrees. These angles span above and below the critical angle for a sapphire/polystyrene interface. The thickness of the polystyrene film was determined using a calibration curve constructed by calculating the integrated optical energy density distribution as a function of incident angle, distance from the prism interface and polymer thickness. The calculations were used to determine the incident angle where waveguide
\end{abstract}


modes are excited within the polymer film, which is the angle with the highest integrated optical energy density. The film thicknesses measured by SA Raman spectroscopy ranged from less than $400 \mathrm{~nm}$ to $1.8 \mu \mathrm{m}$. The average percent uncertainty in the SA Raman determinations for all films was $4 \%$, and the measurements agreed with those obtained from optical interferometery within the experimental uncertainty for all but two films. For the 1270-nm and 580-nm polystyrene films, the SA Raman measurements overestimated the film thickness by 5 and $18 \%$, respectively. The dependence of the calibration curve on excitation polarization and composition of the polymer and bulk layers was evaluated. This preliminary investigation demonstrates that scanning angle Raman spectroscopy is a versatile method applicable whenever the chemical composition and thickness of interfacial polymer layers needs to be measured.

\section{INTRODUCTION}

Interest in the analysis of polymer films continues to grow due to their importance in optics, energy storage and capture devices, microelectronics and the coatings industry.[1-2] Popular optical methods for measuring polymer film thickness include ellipsometry and optical interferometry.[3-4] Like most optical measurements, these methods are non-invasive. However, they do not provide chemical content information. Optical methods that have the ability to provide combined polymer thickness with chemical specificity at an interface have been mostly limited to attenuated total reflection infrared (ATR IR) and total internal reflection (TIR) Raman spectroscopy.[5-9] The penetration depth of the evanescent wave does not vary significantly across the spectrum 
in TIR-Raman spectroscopy since a single excitation wavelength is used. On the other hand, the penetration depth does vary across the infrared spectrum, which complicates ATR IR data analysis. In addition to providing chemical content information, TIRRaman spectroscopy has the benefits of non-invasiveness, speed, limited sample preparation provided the sample can be optically coupled to a prism and the ability to study air, water or organic interfaces.

In TIR-Raman experiments the illuminating laser light is directed onto a prism/analyte interface. Total internal reflection occurs when the incident angle is above the critical angle for the interface, and an evanescent wave that spatially extends into the sample is generated. TIR-Raman spectroscopy is becoming a well established technique for surface sensitive measurements of polymers, plants, and chemical surfactants.[10-13] TIR-Raman spectroscopy has previously been used to analyze thin polymer films.[5, 8 , 14-19] Kivioja et al. analyzed polystyrene on polypropylene films at a single incident angle that was greater than the critical angle.[8] The penetration depth of the evanescent wave under TIR limits the sample thicknesses that can be measured in these experiments. The thickest polystyrene sample studied was $\sim 300 \mathrm{~nm}$, and the TIR-Raman spectroscopy result did not show good agreement with the thickness measured by other optical techniques. However, good agreement was achieved in the 40- to 250-nm thickness range. Varying the incident angle of excitation can provide more information about the sample. Fontaine and Furtak measured the Raman signal from a prism/polymer/air interface at varying incident angles.[15, 20] Their results showed that the intensity of the Raman signal as a function of incident angle could be used to extract the polymer 
thickness, as further outlined below. Their study was limited to a single $1200 \mathrm{~nm}$ film, and they did not present a simple model that could easily be extended to other samples.

Integrated optical waveguides that are approximately the thickness of the excitation wavelength can increase the path length of light by a few orders of magnitude due to multiple total internal reflections within a dielectric.[21] This principle underlies a number of waveguide-based spectroscopies.[22-23] In order to confine the incident light in the dielectric layer the refractive indices of the surrounding media must be lower than the refractive index of the dielectric $\left(n_{1}<n_{2}\right.$, dielectric $\left.>n_{3}\right)$. Radiative or "leaky" waveguides can occur at the interface when $n_{1}>n_{2, \text { dielectric }}>n_{3}$.[20] The optical energy density distributed through the dielectric exhibits an interference pattern at select incident angles at the radiative waveguide interface. There are substantial optical energy density enhancements at angles where constructive interference occurs, and this depends on the thickness of the dielectric layer, among other factors. In the present study scanning angle Raman spectroscopy was used to analyze homogeneous polystyrene films of different thickness, and a simple model for extracting polymer thickness was developed by modeling optical energy density enhancements integrated over the entire polymer film at different incident angles and polymer thicknesses. The experimentally determined incident angle producing the largest Raman signal has been compared to incident angles where waveguide modes are predicted based on the electric field simulations. The goal of this work is to show the benefits of using scanning angle Raman spectroscopy over other optical-based measurements in the analysis of polymer films. The results from this study show facile scanning angle Raman spectroscopy measurements display high signal- 
to-noise ratio spectra that provide combined polymer thickness with tens of nanometer spatial resolution and chemical composition information.

\section{EXPERIMENTAL}

\section{Film preparation}

All chemicals were purchased from Sigma-Aldrich, St. Louis, MO. Polystyrene pellets (molecular weight 280,000) were dissolved in analytical grade toluene at 12, 10, $9,7,6$, or $4 \%$ w/v. Two hundred microliters of the polymer solution were coated onto a 25.4-mm sapphire disc (Meller Optics, Providence, RI) using a KW-4A spin coater (Chemat Technology, Northridge, CA). The films were coated at 3000 RPM for 60 seconds and dried overnight at room temperature. Film thicknesses were confirmed using a F20 thin film measurement system (Filmetrics, San Diego, CA) in transmission mode. The root-mean-square surface roughness measured by tapping mode atomic force microscopy of the 10 and $5 \% \mathrm{w} / \mathrm{v}$ polystyrene films were $1.6 \mathrm{~nm}$ and $0.3 \mathrm{~nm}$, respectively. The root-mean-square surface roughness of the sapphire substrate was 0.8 $\mathrm{nm}$. The atomic force microscopy scan size for all roughness measurements was $25 \mathrm{x} 25$ $\mu \mathrm{m}$.

\section{Sample configuration}

The scanning angle Raman spectroscopy sample configuration is represented in Fig. 1. A sapphire prism was used for all measurements. A custom-made flow cell was designed to hold the hemispherical prism and sapphire substrate on which the polymer films were coated. Immersion oil (Cargille Labs, Cedar Groove, NJ, $n=1.780$ ) was used 
to ensure optical contact between the prism and substrate. During each experiment careful attention was paid to ensure the immersion oil layer was not compromised. The flow cell was cleaned before each experiment by sonication and then dried with a stream of $\mathrm{N}_{2}$ gas.

\section{Instrumentation}

Raman experiments were performed using a previously described scanning angle Raman microscope, which is capable of recording Raman spectra in the incident angle range of $34.5^{\circ}$ to $74.0^{\circ}$.[24] Briefly, the $785 \mathrm{~nm}$, narrow wavelength output laser was directed onto a variable angle mirror mounted onto a motorized translational stage. The beam was focused using a lens mounted on a separate motorized translational stage. The variable angle mirror directs the incident light onto the prism/sample interface that is centered on an inverted microscope. A 1280 x 1024 CMOS camera mounted in the front port of the microscope is used to align and focus the laser beam at the sample interface. The reflectivity was collected using a $1 \mathrm{~cm}^{2}$ photodiode mounted on a third vertically oriented motorized translational stage opposite the variable angle mirror and focusing lens. The Raman scatter was collected with a 10x magnification, 0.30 NA long working distance objective and sent onto a f/1.8i imaging spectrometer fitted with a 1340 x 400 pixel near-infrared enhanced CCD. The motorized translational stages and reflectivity were controlled though an in-house developed program with Labview 8.6.

The excitation power was set to $210 \mathrm{~mW}$ at the prism and the acquisition time was 60 seconds per spectrum. The polarization of the incident light was controlled with a polarizer and a half-wave plate to deliver s- or p-polarized light to the sample interface. 
The reflected light intensity from a sapphire/water interface was collected to calibrate the incident angles by modeling the data with Fresnel calculations using a program developed in IGOR Pro 6.1 that allows the incident angle spread to be varied.[25-26]

Data analysis

Peak areas and intensities for the measured Raman bands were calculated by fitting them to a Gaussian curve with the "multipeak fitting 2" algorithm in IGOR Pro 6.1. Signal-to-noise ratios $(\mathrm{S} / \mathrm{N})$ were calculated as the maximum of the $1001 \mathrm{~cm}^{-1}$ peak intensity after background subtraction divided by the standard deviation of the noise measured from 920 to $950 \mathrm{~cm}^{-1}$ in a region of the spectrum where no analyte peaks were present. A 3-D finite-difference-time-domain (FDTD)-based simulation (EM Explorer, San Francisco, CA) was used to calculate the optical energy density distribution at the interface. The calculations assumed all layers had a constant index of refraction and were homogeneous. The indices of refraction used for the calculations were: sapphire prism $\left(n_{\text {p-polarization }}=1.764, n_{\text {s-polarization }}=1.753\right)$, polystyrene $(n=1.578)$ and air $(n=1.000)$. With the angular resolution used in these experiments, no difference in the polystyrene index of refraction was measured with p- or s-polarized light.

\section{RESULTS AND DISCUSSION}

Enhancement of the scanning angle Raman signal at the thin polymer-air interface

The goals of this work are to experimentally measure and theoretically model the Raman scatter produced from radiative waveguides consisting of thin polystyrene films as a function of incident angle, and to develop a simple model for measuring polymer 
thickness in the $400 \mathrm{~nm}$ to $2 \mu \mathrm{m}$ range. The first step is to analyze the Raman spectrum of polystyrene under different excitation conditions, including the scanning angle Raman sample geometry shown in Fig. 1. Fig. 2 shows the bulk Raman spectrum of solid (i.e., not cast as a thin film) polystyrene (black), the scanning angle Raman spectrum of a 580$\mathrm{nm}$ polystyrene film at an angle lower than the sapphire/polystyrene critical angle (light gray) and an angle higher than the critical angle (dark gray). In all spectra, peaks representative of polystyrene are present. The aromatic ring breathing $\left(1001 \mathrm{~cm}^{-1}\right), \mathrm{C}-\mathrm{H}$ bending $\left(1032 \mathrm{~cm}^{-1}\right)$, ring deformation $\left(620 \mathrm{~cm}^{-1}\right)$, and $\mathrm{C}=\mathrm{C}$ aromatic ring stretching $\left(1602 \mathrm{~cm}^{-1}\right)$ modes are the most intense bands.[27] The two additional peaks in the scanning angle Raman spectra at 645 and $750 \mathrm{~cm}^{-1}$ are from the sapphire prism. Comparing the bulk spectrum and the scanning angle Raman spectra in Fig. 2, differences in the relative peak intensity are present. The peak intensity depends on the depolarization ratio of the peak and the excitation polarization, as previously discussed.[24]

While the conventional backscattering configuration provides chemical information generated from the bulk material, it lacks the sensitivity to measure thin films. The spectrum shown in the inset to Fig. 2 was collected using the 580-nm polystyrene film and a backscattering configuration. Unlike the scanning angle Raman spectra in the same figure, no peaks were recorded despite measuring the same polystyrene thickness. The scanning angle Raman spectra at an incident angle of 60.0 degrees (Fig. 2, light grey) and 64.1 degrees (Fig. 2, dark grey) exhibit signal enhancement relative to probing the same amount of analyte using a traditional backscattering geometry. With an incident angle spread of 0.5 degrees the critical angle 
for a sapphire/polystyrene interface is 63.8 degrees. The background subtracted Raman intensity is 10.7 -fold higher at 60.0 degrees compared to 64.1 degrees, and this is explained by the optical energy density enhancements when waveguide modes are excited in the film. The $\mathrm{S} / \mathrm{N}$ ratios of the scanning angle Raman spectra are 119 and 16.3 above and below the sapphire/polystyrene critical angle, respectively.

\section{Raman signal at different incident angles and polarization of the exciting light}

Fig. 3 shows 2D plots of Raman signal as a function of Raman shift and incident angle for a 950-nm polystyrene film with (a) s-polarized or (b) p-polarized excitation. It is evident from the plots that the Raman signal is higher at certain incident angles below the 63.8-degree sapphire/polystyrene critical angle. Cross sections of the $1001 \mathrm{~cm}^{-1}$ peak at different incident angles are shown in the figure insets. The angle where the Raman signal is the most intense depends on the excitation polarization and polymer thickness. The angle producing the most intense Raman scatter is $60.4^{\circ}$ or $60.6^{\circ}$ for s- or $\mathrm{p}$ polarized excitation, respectively. These angles are in good agreement to the maximum values predicted by integrating the calculated optical energy density over the entire film thickness (solid lines in the Fig. 3 inset). At angles higher than the 63.8-degree sapphire/polystyrene critical angle, the collected Raman scatter decays as expected, and is less intense than at lower angles where waveguide modes are excited.[28]

The number of intense peaks in the Raman spectra varies for s- and p-polarized excitation (Fig. 3). The p-polarized spectra exhibit intense peaks at 1602, 1181, 1151 and $1001 \mathrm{~cm}^{-1}$. In contrast, only the $1001 \mathrm{~cm}^{-1}$ peak has significant intensity in the spolarization spectra. The peaks that only have an appreciable intensity with p- polarized 
excitation are depolarized bands; while the $1001 \mathrm{~cm}^{-1}$ peak is a polarized band. Despite only one intense peak in the spectra, the background subtracted $1001 \mathrm{~cm}^{-1}$ peak area is 2.4-fold higher with s- compared to p-polarized excitation. All data that follows were collected using p-polarized excitation since the $\mathrm{S} / \mathrm{N}$ was sufficient, and more peaks could be analyzed across the spectra. Although separate calculations would be required to model the Raman signal, either p- or s-polarized excitation (or both) could be used to collect the Raman scatter. The benefit of using both polarizations is that additional details about the structure and orientation of the polymer may be obtained by the polarization dependence of the Raman signal.

Calculated integrated optical energy density distributions for different polystyrene thicknesses

In order to determine the incident angle where waveguide modes are excited for interfaces containing different polystyrene thicknesses, calculations were performed for films ranging from $200 \mathrm{~nm}$ to $2 \mu \mathrm{m}$. A subset of the calculated data are shown in Fig. 4 . The right panel shows $2 \mathrm{D}$ plots of the integrated optical energy density as a function of distance from the prism interface, with the interface designated at the distance $1000 \mathrm{~nm}$, over a 58 to 66-degree incident angle range. This angle range was selected for a majority of the polymer thicknesses to minimize computing time, and was extended down to 54 degrees for films less than $600 \mathrm{~nm}$ thick. The 2D electric field distribution plot can be used to determine angles where waveguide modes are excited and specific locations within the polymer film where the bulk of the Raman signal is generated. Waveguide enhancements require polymer films with thicknesses greater than $\sim 250 \mathrm{~nm}$ using 785 - 
nm excitation. Fig. 4 f represents a $200-n m$ polystyrene film. Within the analyzed angle range, no discernible waveguide pattern can be observed, and the majority of the Raman signal is predicted to come from the polystyrene in contact with the sapphire substrate. In contrast, films with a thickness greater than a few hundred nanometers exhibit a more complex spatial distribution, and a majority of the Raman signal will be generated in approximately the middle of the film, away from the interfaces. The surface sensitivity is limited using angles below the sapphire/polystyrene critical angle. Experimentally the only Raman signal generated is form the polystyrene film since the semi-infinite air layer used in the measurements does not produce a Raman signal.

The optical energy density integrated over the entire film thickness is directly related to the predicted intensity of the Raman scatter.[18] The left panel in Fig. 4 displays the integrated optical energy density generated from the entire polystyrene film over the incident angle range from 58 to 66 degrees. Films with a thickness of a few hundred nanometers up to approximately $1200 \mathrm{~nm}$ have a single maximum calculated in this incident angle range, while films thicker than $1200 \mathrm{~nm}$ exhibit two or more maxima. In cases where multiple waveguide modes are present, only the mode at the largest incident angle was considered. For example, the peak maximum of the 950-nm radiative waveguide is at 60.4 degrees (Fig. 4c) and the right-most peak maximum of the $1850-\mathrm{nm}$ polystyrene film is at 62.8 degrees (Fig. 4a).

Calculated angular shifts in the radiative waveguide's integrated optical energy density maxima can be compared to experimental angular shifts in the intensity of the Raman scatter to determine the polymer film thicknesses. These shifts can indicate thickness changes on the nanometer level. Calculated values predict a 0.10 degree shift 
between a $1230 \mathrm{~nm}$ film and $1200 \mathrm{~nm}$ film that can be detected with experimental data of sufficient $\mathrm{S} / \mathrm{N}$ and similar angular resolution.

A calibration curve was constructed using the calculated integrated optical energy density maxima for polymer thicknesses in the 400 to $2 \mu \mathrm{m}$ range (Fig. 5). Thinner films, down to $\sim 250 \mathrm{~nm}$ could be included in the calibration curve. This would require extending the incident angle range of the calculations, which would increase calculation times as discussed above. Similarly, thicker films could be included in the calibration plot. As the polymer thickness increases, the interference pattern as a function of incident angle becomes more complex, and this requires analyzing the sample at higher angular resolution. In this case, experimental time would increase. The calibration points were fit with a double exponential curve $y=\left(30.6 \cdot \mathrm{e}^{0.049 \cdot \mathrm{x}}\right)+\left(1.44 \cdot 10^{-13} \cdot \mathrm{e}^{0.585 \cdot \mathrm{x}}\right)$ to an $\mathrm{R}^{2}$ value of 0.9899 . The minimum in the residual plot is at a thickness of $1200 \mathrm{~nm}$ and the maximum is at $2 \mu \mathrm{m}$. The predicted percent uncertainties at these thicknesses are -4 and 3\%, respectively, and the maximum expected percent uncertainty is $\sim 5 \%$ for $400 \mathrm{~nm}$ films.

\section{Experimental determination of polymer waveguide thickness}

Fig. 6 shows the area of the $1001 \mathrm{~cm}^{-1}$ polystyrene peak for the indicated incident angles and six polystyrene films fabricated by spin coating 4 to $12 \% \mathrm{w} / \mathrm{v}$ polystyrene in toluene onto sapphire substrates. Spectra were collected every 0.4 degrees for most interfaces, and no lower than 0.25 degrees or higher than 0.5 degrees for all interfaces. The experimental Raman scatter plots were fit to a Gaussian curve to determine the angle corresponding to the maximum intensity. An example fit is shown in the inset to Fig. 6c. 
Similar to the calculated plots shown in Fig. 4, as the thickness of the polystyrene films decreases, the Raman scatter versus incident angle plot broadens and the incident angle that produces the maximum Raman scatter shifts to lower incident angles. The experimental data show distinct maxima for all but the $4 \% \mathrm{w} / \mathrm{v}$ polystyrene films, and multiple waveguide modes are recorded for the $12 \% \mathrm{w} / \mathrm{v}$ polystyrene film within this incident angle range. The overall Raman intensity increases as the polymer thickness increases, which is consistent with probing more polymer.

The polystyrene film thickness was calculated using the angle that produced the most intense Raman scatter from the Gaussian fit and the fit equation obtained from the calibration. The results are shown for all the films in Fig. 7. The uncertainty in the scanning angle Raman measurements ranges from 1.6 to $6.7 \%$. Also shown in this figure are the thicknesses determined using optical interferometry for films fabricated using the same spin coating procedure. The values obtained by scanning angle Raman spectroscopy are well correlated to the optical interferometer measurements, and in all but two cases the values reported for the two methods agree within the experimental uncertainty. For the 1270-nm and 580-nm films, the SA Raman measurements overestimated the film thickness by 5 or $18 \%$, respectively. The average difference between the optical interferometery and SA Raman spectroscopy results is $6.9 \%$. Some of this difference is the result of having to analyze different films by the two techniques. Altering the thickness range of the calibration plot used for the SA Raman measurements did not significantly alter the average difference obtained by the two methods. The ability to probe several incident angles with scanning angle Raman spectroscopy allows thickness measurements of polystyrene films ranging from $2 \mu \mathrm{m}$ to less than $400 \mathrm{~nm}$. 
When combined with traditional total internal reflection Raman measurements using the same sample geometry shown in Fig. 1, the range could decrease to $\sim 50 \mathrm{~nm}$.[8] Since the spectral $\mathrm{S} / \mathrm{N}$ ratio is lower at angles above the critical angle, acquisition times need to be longer than those used for these experiments.

The measured signal enhancement in the scanning Raman geometry depends on the medium adjacent to the polymer film (i.e., Fig. 1 medium 3). Although the analyses were performed using air as the third medium, water or organic solvents can in principle be used provided the integrity of the polymer film is not compromised. In order to understand how this will affect the Raman signal, the integrated optical energy density was calculated for water $\left(\mathrm{n}_{3}=1.329\right)$ or organic $\left(\mathrm{n}_{3}=1.700\right)$ layers. At the sapphire/2$\mu \mathrm{m}$ polystyrene film/medium 3 interface, the Raman scatter is expected to be 0.7-times (organic) or 0.3-times (water) lower compared to the value measured in air, but the constructive interface pattern as a function of varying incident angle is expected to be the same to at least 0.4 -degree angular resolution. This means the calibration curve shown in Fig. 5 is valid for these conditions and interfaces with other $n_{3}$ layers.

\section{CONCULSIONS}

A scanning angle Raman spectroscopy calibration curve for determining radiative polymer waveguide thickness with p-polarized, 785-nm excitation was constructed. The calibration curve will be suitable for any polymer with a similar index of refraction to that of polystyrene, and similar methods can be used to construct a calibration for polymers of other indices of refraction, excitation wavelengths or polarizations. The optical energy density distribution as a function of incident angle and distance from the 
prism interface can be used to determine the spatial dependence of the Raman signal, which is primarily generated from the center of the polymer film when the thickness is more than $\sim 250 \mathrm{~nm}$. The success of the presented approach is dependent on the incident angle resolution and the spectral S/N. Sixty second acquisition times were used to collect each Raman spectrum every 0.25 to 0.5 degrees within the range 56 to 70 degrees. These conditions were sufficient for close agreement to an independent method for determining polymer thickness in the $400 \mathrm{~nm}$ to $2 \mu \mathrm{m}$ range. While the reported uncertainty of the SA Raman spectroscopy thickness measurements is slightly higher than that of optical interferometry for many of the samples, longer acquisition times or averaged accumulations, and higher angular resolution measurements will reduce the uncertainty. The presented method will have utility for many applications were both polymer thickness and chemical content need to be measured, especially with stacked polymer layers.

\section{ACKNOWLEDGEMENTS}

This research is supported by the U.S. Department of Energy, Office of Basic Energy Sciences, Division of Chemical Sciences, Geosciences, and Biosciences through the Ames Laboratory. The Ames Laboratory is operated for the U.S. Department of Energy by Iowa State University under Contract No. DE-AC02-07CH11358. 


\section{REFRENCES}

[1] L. Eldada, L.W. Shacklette, Ieee J. Sel. Top. Quant., 6 (2000) 54-68.

[2] Y. Wang, A. Hongo, Y. Kato, T. Shimomura, D. Miura, M. Miyagi, Appl.

Opt., 36 (1997) 2886-2892.

[3] Y. Ebata, A.B. Croll, A.J. Crosby, Soft Matter, (2012) 9086-9091.

[4] A. Knoll, R. Magerle, G. Krausch, J. Chem. Phys., 120 (2004) 1105-1116.

[5] N.A. Nikonenko, O.N. Tretinnikov, J. Appl. Spectrosc., 75 (2008) 878-882.

[6] P. Yang, X.F. Meng, Z.Y. Zhang, B.X. Jing, J. Yuan, W.T. Yang, Anal.

Chem., 77 (2005) 1068-1074.

[7] L.J. Fina, Appl. Spectrosc. Rev., 29 (1994) 309-365.

[8] A.O. Kivioja, A.S. Jaaskelainen, V. Ahtee, T. Vuorinen, Vib. Spectrosc., 61 (2012) 1-9.

[9] M.W. Meyer, McKee, K.J., Nguyen, H.T., Smith, E.A., J. Phys. Chem. C, 116 (2012) 24987-24992.

[10] R. Iwamoto, K. Ohta, M. Miya, S. Mima, Appl. Spectrosc., 35 (1981) 584587.

[11] D.A. Woods, C.D. Bain, Analyst, 137 (2012) 35-48.

[12] F. Ishizaki, M. Kim, Jpn. J. Appl. Phys., 47 (2008) 1621-1627.

[13] K.J. McKee, M.W. Meyer, E.A. Smith, Anal. Chem., 84 (2012) 9049-9055.

[14] J.T. Ives, W.M. Reichert, J. Appl. Polym. Sci., 36 (1988) 429-443.

[15] N.H. Fontaine, T.E. Furtak, J. Opt. Soc. Am. B., 14 (1997) 3342-3348.

[16] N.E. Schlotter, J.F. Rabolt, Appl. Spectrosc., 38 (1984) 208-211.

[17] N.E. Schlotter, J.F. Rabolt, J. Phys. Chem., 88 (1984) 2062-2067. 
[18] C.G. Zimba, V.M. Hallmark, S. Turrell, J.D. Swalen, J.F. Rabolt, J. Phys. Chem., 94 (1990) 939-943.

[19] D.R. Miller, O.H. Han, P.W. Bohn, Appl. Spectrosc., 41 (1987) 245-248.

[20] N.H. Fontaine, T.E. Furtak, Phys. Rev. B., 57 (1998) 3807-3810.

[21] J.T. Bradshaw, S.B. Mendes, S.S. Saavedra, Anal. Chem., 77 (2005) 28a36a.

[22] I. Chabay, Anal. Chem., 54 (1982) 1071-1080.

[23] A. Abbas, M.J. Linman, Q. Cheng, Sensor Actuat. B, 156 (2011) 169-175.

[24] K.J. McKee, M.W. Meyer, E.A. Smith, Anal. Chem., 84 (2012) 4300-4306.

[25] W.N. Hansen, J. Opt. Soc. Am., 58 (1968) 380-388.

[26] S.N. Kasarova, N.G. Sultanova, C.D. Ivanov, I.D. Nikolov, Optical Materials, 29 (2007) 1481-1490.

[27] R.L. McCreery, Raman Spectroscopy for Chemical Analysis, in, WileyInterscience, New York, NY, 2000, pp. 263.

[28] K.J. McKee, E.A. Smith, Rev Sci Instrum, 81 (2010) 043106-043106043106. 


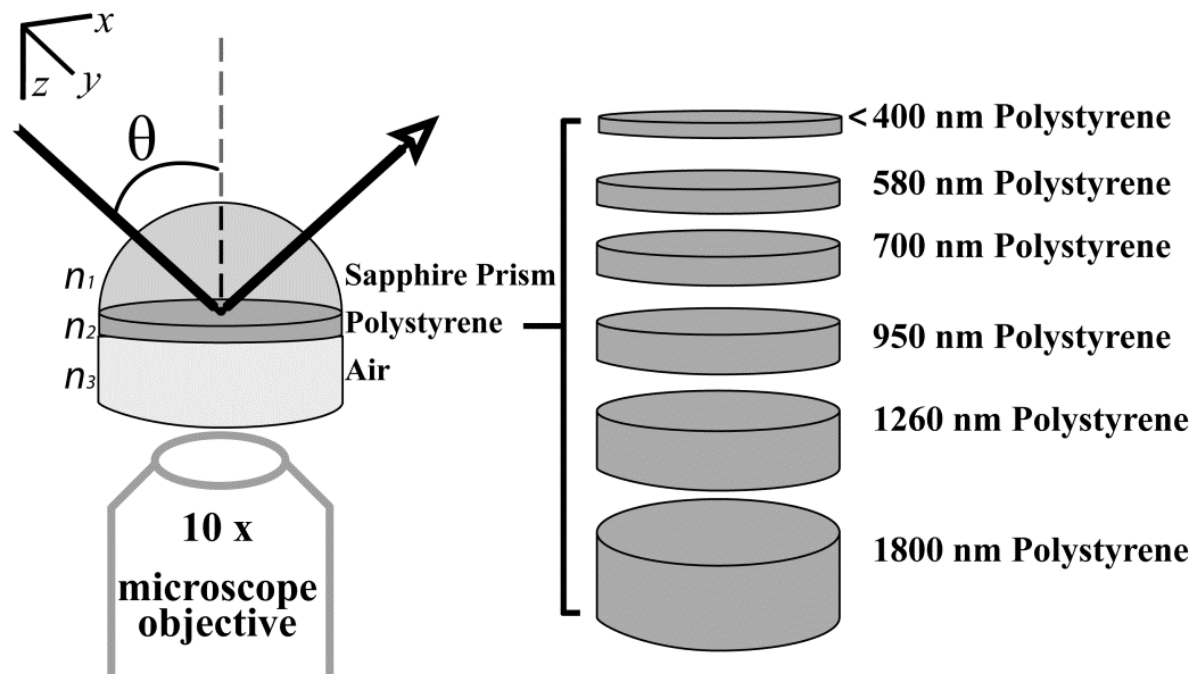

Fig. 1. Schematic of the scanning angle Raman spectroscopy sample geometry used to measure polystyrene film thicknesses. The incident angle $(\theta)$ of the excitation light at the interface is varied from 56 to 70 degrees in approximately 0.4 degree increments while simultaneously collecting Raman spectra. 


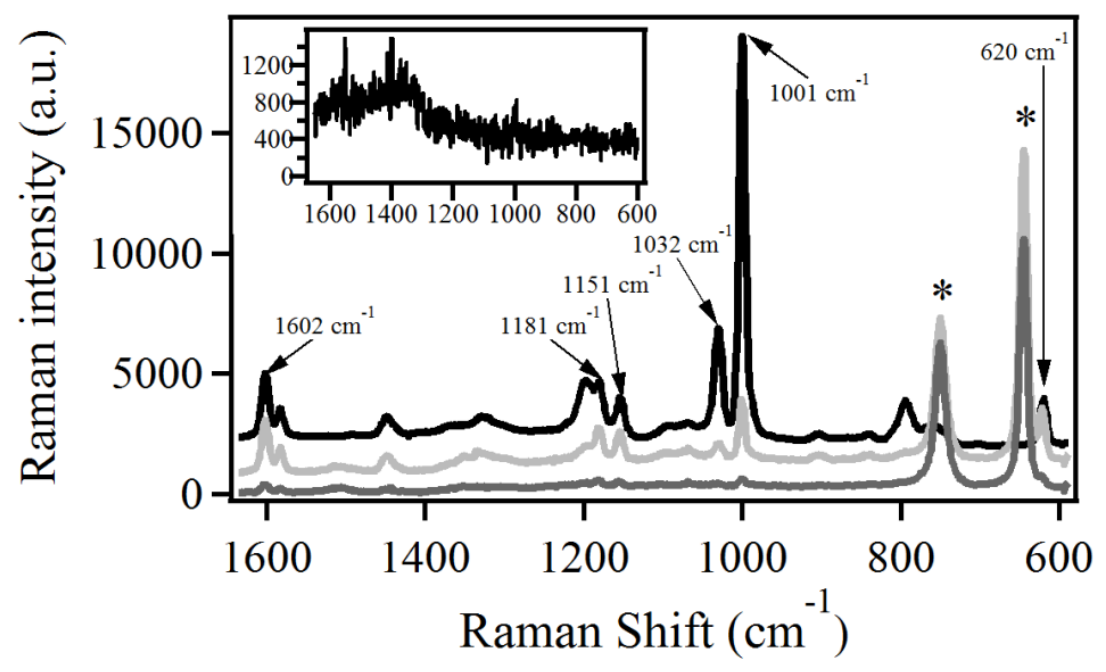

Fig. 2. Bulk Raman spectrum of solid polystyrene (black) collected using the traditional $180^{\circ}$ backscattering Raman spectroscopy geometry. Scanning angle Raman spectra of a thin 580-nm polystyrene film collected with an incident angle of 60.0 (light grey) and 64.1 degrees (dark grey). The critical angle for a sapphire/polystyrene interface is 63.8 degrees. The peaks from the sapphire prism are marked with an asterisk $\left(^{*}\right)$. Inset: Raman spectrum of the same $580-\mathrm{nm}$ polystyrene film measured with the traditional $180^{\circ}$ backscattering configuration. All spectra were collected using p-polarized excitation. 


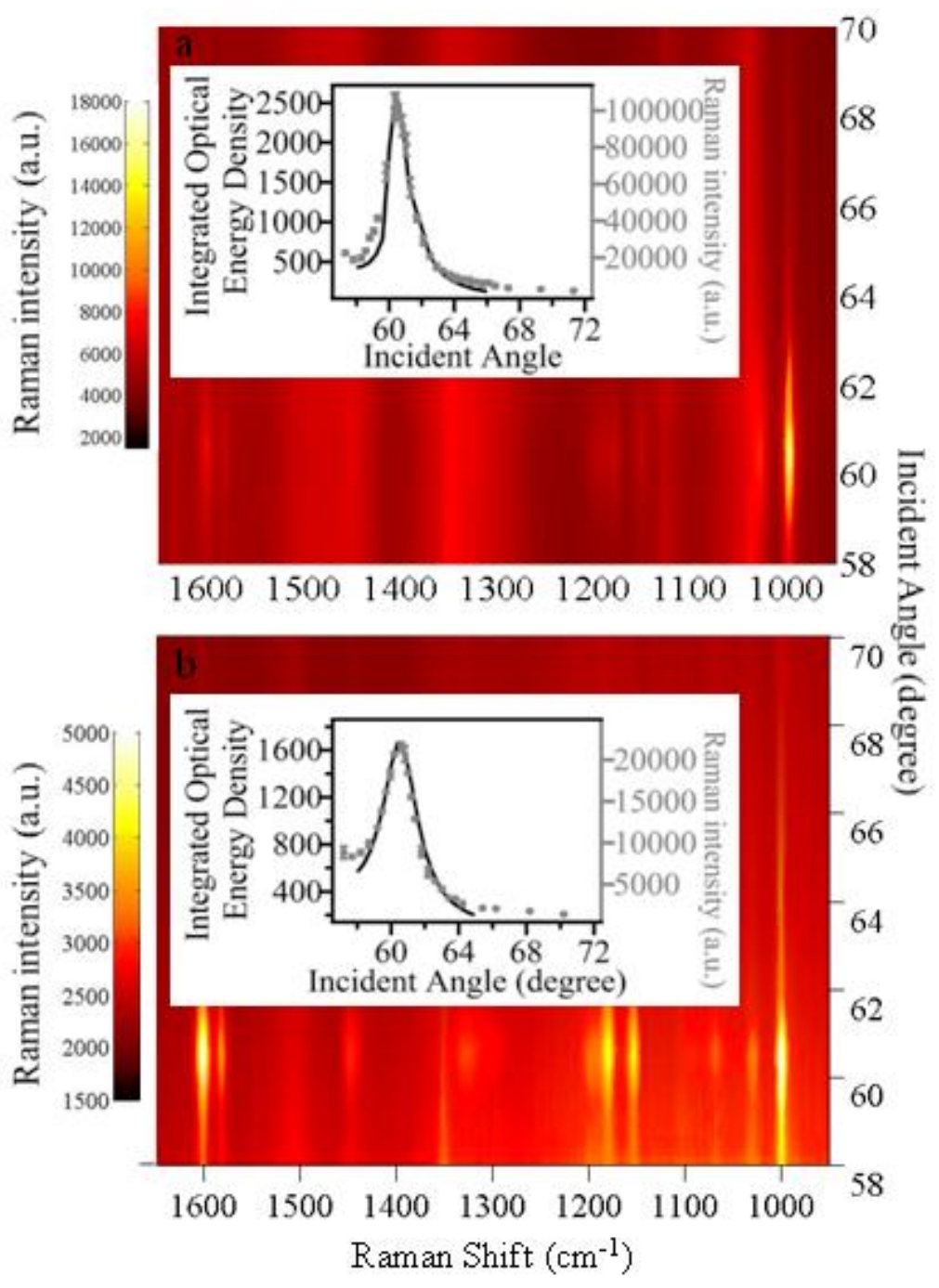

Fig. 3. Two dimensional plot of the Raman scatter as a function of Raman shift and incident angle using (a) s- polarized or (b) p- polarized incident light and a $9 \%(\mathrm{w} / \mathrm{v})$ polystyrene film. Insets: (grey symbol) experimental Raman scatter of the $1001 \mathrm{~cm}^{-1}$ peak across all incident angles and (black line) the calculated integrated optical energy density using 785-nm excitation, 950-nm polymer thickness, (a) s-polarized excitation $\left(E y^{2}\right),(b)$ p-polarized excitation $\left(E z^{2}+E x^{2}\right)$. 


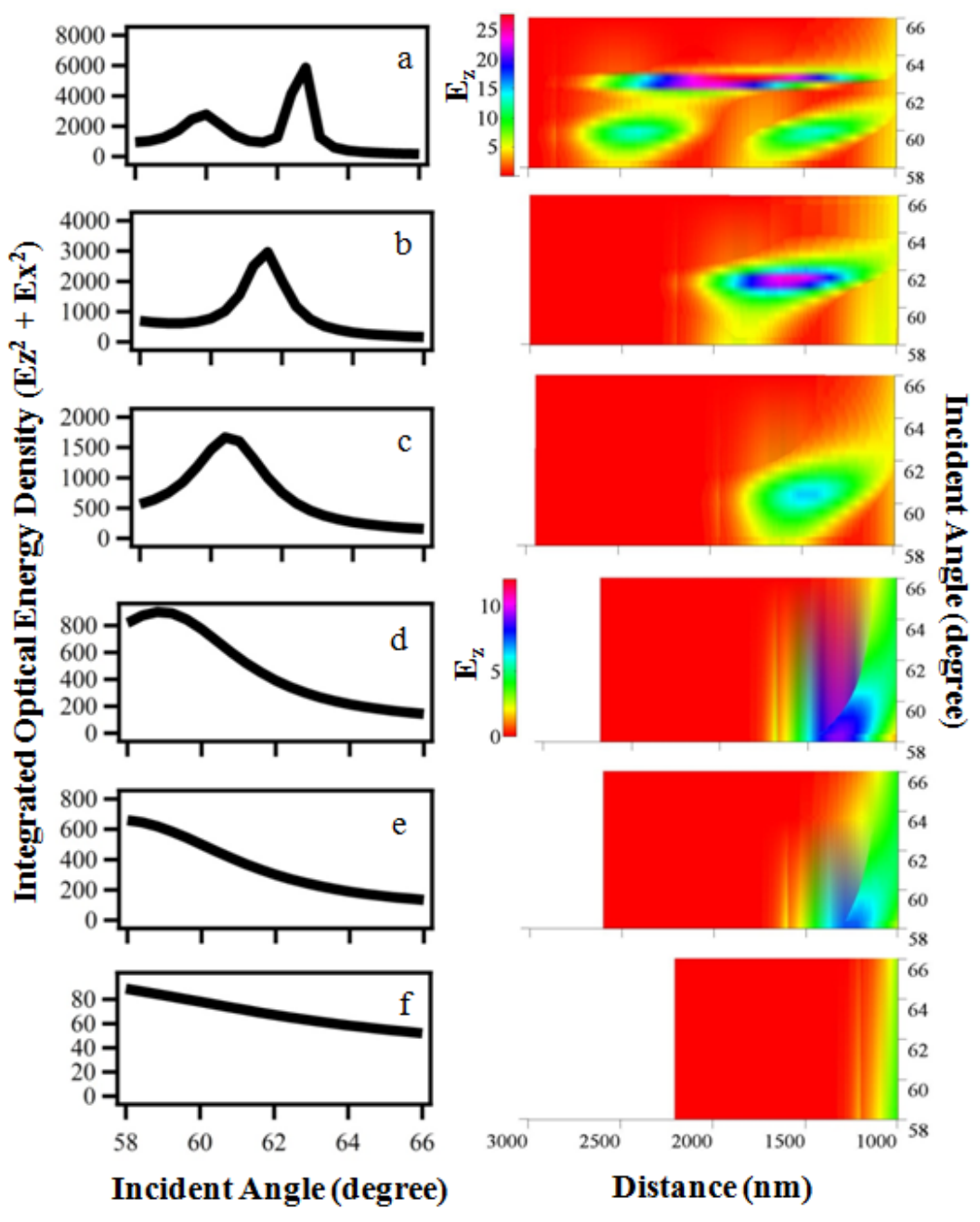

Fig. 4. Calculated integrated optical energy density over the entire film thickness as a function of incident angle (left column) with an angle resolution of 0.4 degrees, and a 2D plot of the electric field distribution as a function of incident angle and the distance away from the sapphire/polymer interface (right column) with the interface designated at 1000 $\mathrm{nm}$ for polymer ( $n=1.578$ ) film thickness: (a) $1800 \mathrm{~nm}$, (b) $1200 \mathrm{~nm}$, (c) 950, (d) $725 \mathrm{~nm}$, (e) $600 \mathrm{~nm}$ and (f) $200 \mathrm{~nm}$ (right column). The intensity scale is the same for the top three plots and the bottom three plots. 


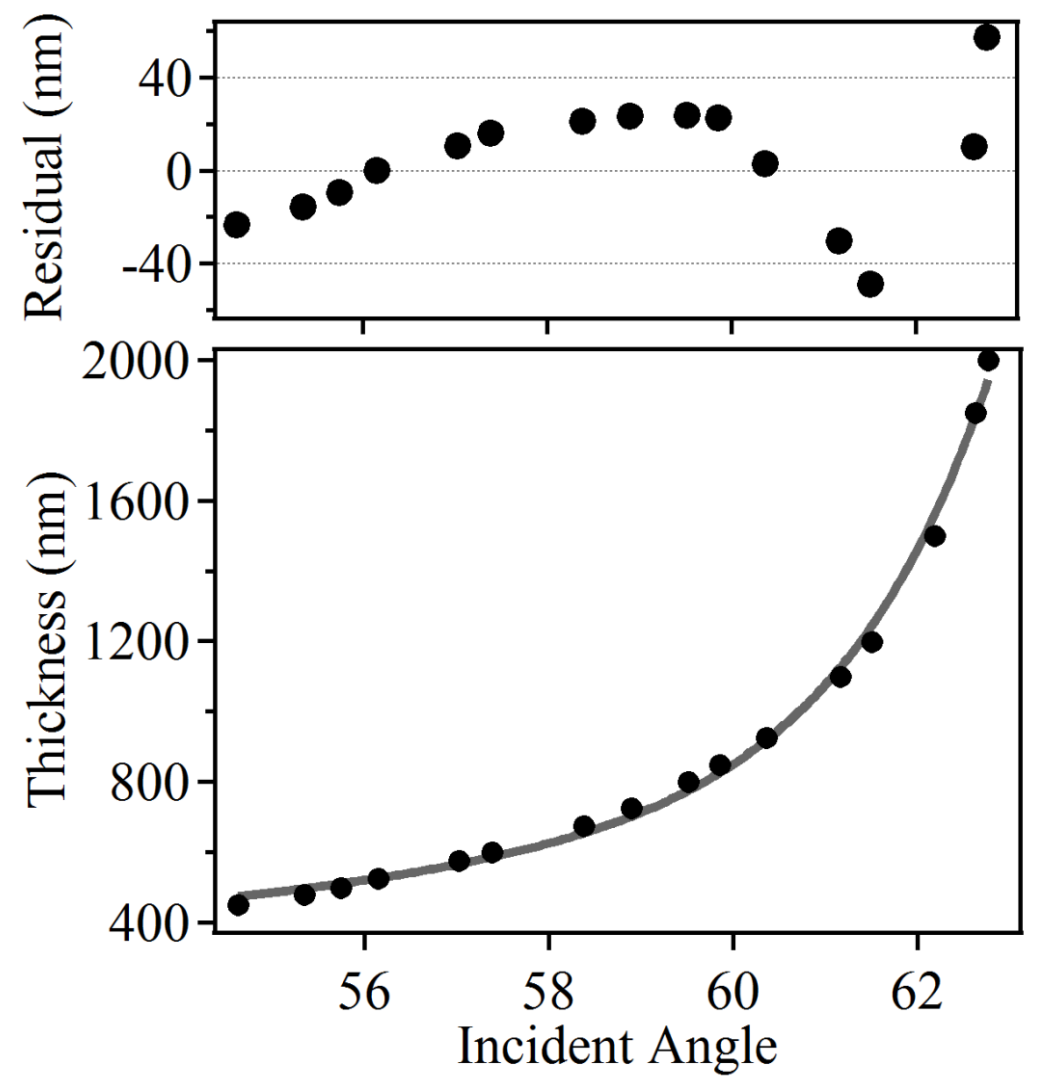

Fig. 5. Calibration plot for the polymer thickness as a function of the incident angle producing the maximum Raman scatter of the $1001 \mathrm{~cm}^{-1}$ polystyrene peak. Experimental data (black symbol) were fit to a double exponential curve (grey line) to generate the fit equation shown in the text. The upper plot shows the residual between the experimental points and the double exponential fit. 


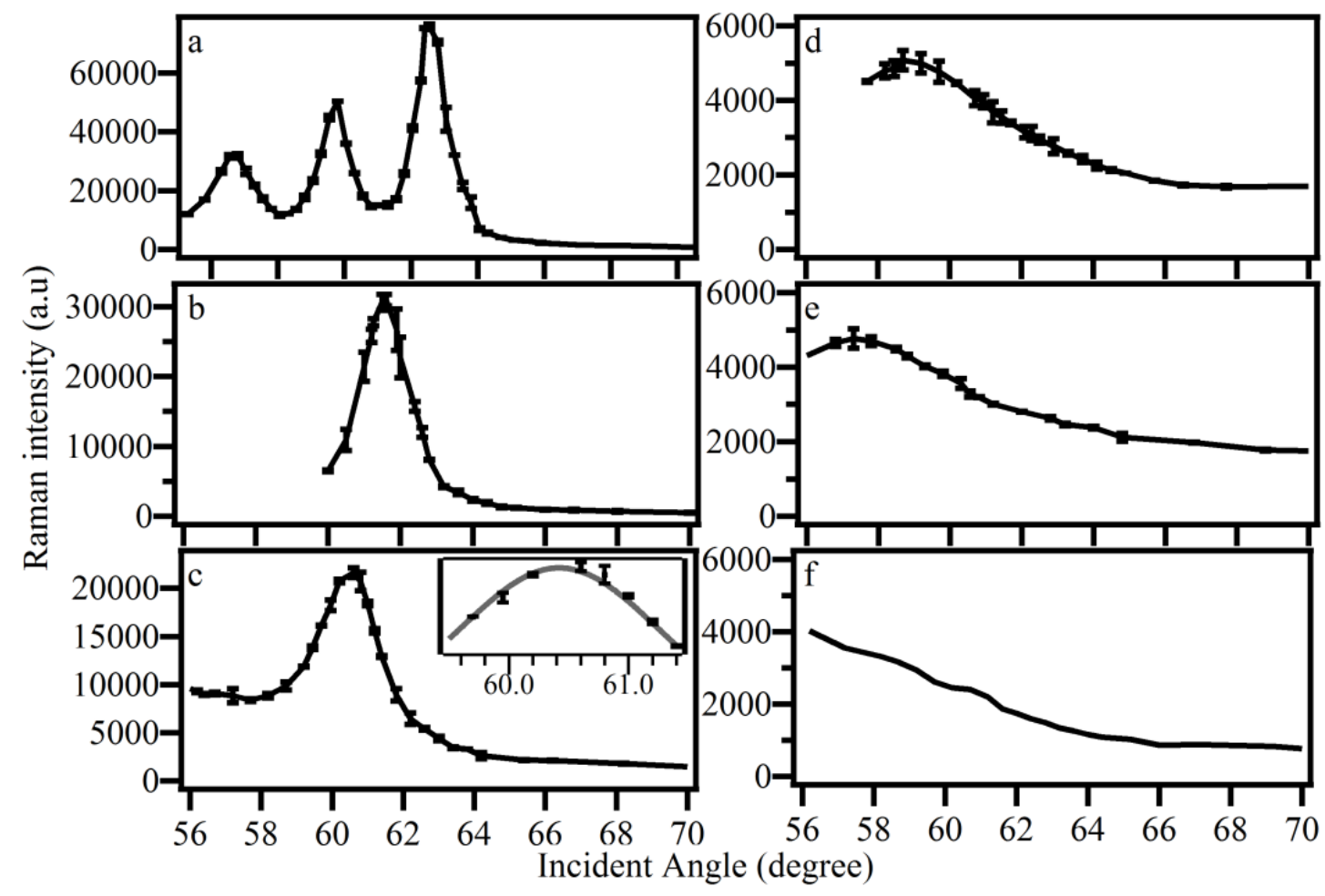

Fig. 6. Experimental Raman scatter of the polystyrene $1001 \mathrm{~cm}^{-1}$ ring breathing mode as a function of incident angle for thin films spin coated from a polystyrene solution of (a) 12, (b) 10, (c) 9, (d) 7, (e) 6 or (f) $4 \%$ w/v in toluene. Error bars are included on all data except panel f and represent the standard deviation from duplicate Raman measurements from each film. (c) The inset shows a representative weighted Gaussian fit to the experimental Raman scatter for the $9 \% \mathrm{w} / \mathrm{v}$ polystyrene film. 


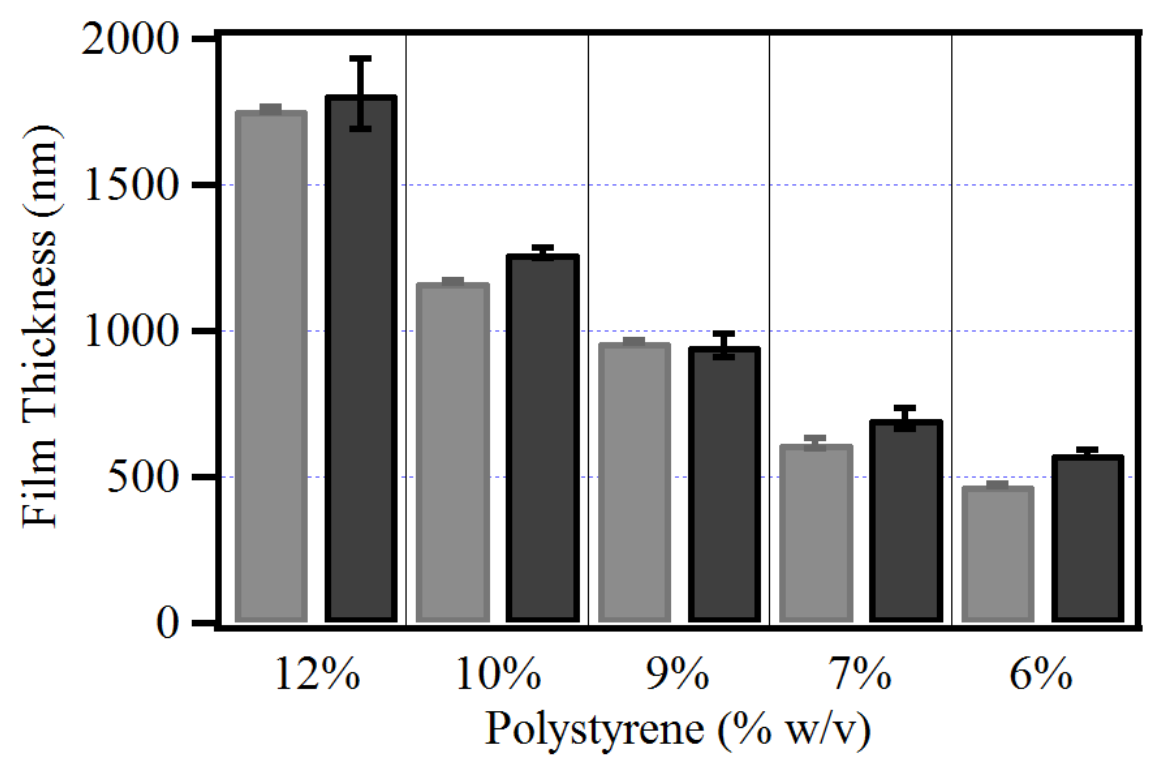

Fig. 7. Polystyrene thickness measured by scanning angle Raman spectroscopy (black) or optical interferometry (grey) for films coated from solutions with the indicated polystyrene concentration. A different set of films were measured by each technique using the same fabrication parameters. Error bars represent the standard deviation for duplicate Raman and triplicate interferometry measurements of the polystyrene film. 


\title{
CHAPTER 3: SCANNING ANGLE PLASMON WAVEGUIDE RESONANCE RAMAN SPECTROSCOPY FOR THE ANALYSIS OF THIN POLYSTYRENE FILMS
}

A paper published in Journal of Phys. Chem. C., 2012, 47, 24987-24992.

Matthew W. Meyer, Kristopher J. McKee, Vy H.T. Nguyen and Emily A. Smith

Ames Laboratory, U. S. Department of Energy, Ames, Iowa 50011-3111, and

Department of

Chemistry, Iowa State University, Ames, Iowa 50011-3111

\begin{abstract}
Scanning angle (SA) Raman spectroscopy was used to characterize thin polymer films at a sapphire/50 nm gold film/polystyrene/air interface. When the polymer thickness is greater than $\sim 260 \mathrm{~nm}$, this interface behaves as a plasmon waveguide; Raman scatter is greatly enhanced with both p- and s-polarized excitation compared to an interface without the gold film. In this study, the reflected light intensities from the interface and Raman spectra were collected as a function of incident angle for three samples with different polystyrene thicknesses. The Raman peak areas were well modeled with the calculated
\end{abstract}


mean-square electric field (MSEF) integrated over the polymer film at varying incident angles. A $412 \mathrm{~nm}$ polystyrene plasmon waveguide generated $3.34 \times$ the Raman signal at $40.52^{\circ}$ (the plasmon waveguide resonance angle) compared to the signal measured at $70.4^{\circ}$ (the surface plasmon resonance angle). None of the studied polystyrene plasmon waveguides produced detectable Raman scatter using a $180^{\circ}$ backscatter collection geometry, demonstrating the sensitivity of the SA Raman technique. The data highlight the ability to measure polymer thickness, chemical content, and, when combined with calculations of MSEF as a function of distance from the interface, details of polymer structure and order. The SA Raman spectroscopy thickness measurements agreed with those obtained from optical interferometery with an average difference of $2.6 \%$. This technique has the potential to impact the rapidly developing technologies utilizing metal/polymer films for energy storage and electronic devices.

\section{INTRODUCTION}

Total internal reflection (TIR) Raman spectroscopy is an analytical technique used to measure chemical content near an interface..$^{1-3}$ In a typical TIR Raman experiment the illuminating light is directed onto a prism/sample/bulk interface at a precise incident angle. ${ }^{4}$ At angles above the critical angle, total internal reflection conditions occur, and a surface-sensitive evanescent wave is generated in the sample. TIR-Raman spectroscopy has been useful in studying films, surfactants, plants, and adsorbates at various interfaces. $^{5-8}$ As with conventional Raman spectroscopy, TIR-Raman spectroscopy has the benefits of being noninvasive, fast, and requiring minimal sample preparation provided the sample can be optically coupled to the prism. For conditions where the same 
amount of analyte is probed, the TIR-Raman geometry acts as a signal enhancement mechanism, and excellent signal-to-noise ratio spectra are possible for thin films using several second acquisition times.

TIR-Raman spectroscopy has been combined with surface plasmon resonance (SPR-Raman spectroscopy) using thin noble metal films to provide predictable signal enhancements, high experimental reproducibility, and the ability to accurately model data with theoretical calculations. ${ }^{9-11}$ An example interface used for SPR-Raman spectroscopy is prism/gold/ sample/bulk medium. The SPR phenomenon occurs at incident angles where propagating surface-plasmon-polaritons are excited in the metal film. Under these conditions, the generated evanescent wave extends spatially into the sample. This field can produce Raman scatter from the molecules located within $\mathrm{d}_{\mathrm{p}} / 2$, where $d_{p}$ is the penetration depth of the evanescent wave. ${ }^{4}$ McKee et al. used SPRRaman spectroscopy for reproducible Raman enhancements ranging from $4.7 \times$ to $3.7 \times$ for aqueous pyridine and nitrobenzene at a sapphire/gold/sample interface compared to a sapphire/sample interface. ${ }^{12}$ The latter signal is already approximately an order of magnitude greater than probing the same amount of analyte using normal illumination geometries. The enhancements from the smooth gold films allowed for the detection of single monolayers of benzenethiol and 4-mercaptopyridine with nonresonant excitation.

Thin polymer films are critical components of many devices including sensors, coatings, and medical implants. ${ }^{13}$ Common optical methods for measuring thin polymer films include ellipsometry, optical interferometry, attenuated total internal reflection infrared spectroscopy, and TIR-Raman spectroscopy. ${ }^{14-16}$ TIR-Raman spectroscopy 
provides the advantage of measuring thickness, structure, and chemical content simultaneously, at a variety of interfaces. ${ }^{17-19}$ TIR-Raman spectroscopy was used to study thin polymer films at a glass interface. ${ }^{20,21}$ Most recently, Kivioja et al. used TIRRaman spectroscopy at a fixed incident angle to measure thin polystyrene films on a sapphire interface. ${ }^{22}$

Optical waveguides can be used to confine incident light within a sample or carry incident light to the sample for the analysis of thin films. ${ }^{23}$ When a polymer film of approximately $\lambda / 2 \eta$ ( $\lambda$ wavelength of excitation, $\eta$ index of refraction of the sample) thickness is coated on a surface plasmon supporting metal film with a bulk air layer, the polymer can act as a radiative or "leaky" waveguide. ${ }^{24,25}$ Recently, leaky waveguides have been used as biosensors for clinical diagnosis and bacterial analysis. ${ }^{24,26}$ Kanger et al. used waveguides in Raman spectroscopy experiments to measure the orientation of porphyrin monolayers. ${ }^{27}$ Their results showed the preferred orientation of the molecules can be obtained using deviations of the monolayer's depolarization ratios from those measured in the bulk. Zimba et al. calculated the expected effects of excitation wavelength on waveguide Raman spectra at a quartz/polymer interface. ${ }^{28}$

At the plasmon waveguide interface, large increases in the interfacial mean square electric field relative to the incident field (MSEF) are generated at incident angles where plasmon waveguide resonances (PWR) are excited. ${ }^{29}$ Until recently, a majority of PWR spectroscopy measurements only utilized the reflected light intensity from the interface. ${ }^{30}$ At the PWR angles, a sharp attenuation of the reflected light occurs with both p- and spolarized incident light. When PWR and Raman spectroscopies are combined (PWR 
Raman spectroscopy), strong enhancements of the Raman signal are expected at the PWR angle(s) due to enhancements in the MSEF. The incident angle(s) where plasmon waveguide resonances are excited depends on the thickness of the polymer layer; thus, polymer films of different thickness should generate unique patterns of Raman scattering intensities as the incident angle is scanned. One advantage of PWR Raman spectroscopy, compared to SPR Raman spectroscopy is that both p-polarized light (electric field oriented parallel to the plane of incidence) and s-polarized (perpendicular) light can be used to produce MSEF in the $\mathrm{X}, \mathrm{Y}$, and $\mathrm{Z}$ direction $\left(\mathrm{MSEF}_{\mathrm{X}}, \mathrm{MSEF}_{\mathrm{Y}}, \mathrm{MSEF}_{\mathrm{Z}}\right)$, where $\mathrm{X}$ and $\mathrm{Y}$ extend in the focal plane and $\mathrm{Z}$ is perpendicular to the focal plane. In SPR-Raman spectroscopy no $\mathrm{MSEF}_{\mathrm{Y}}$ component is generated.

The goals of this study are to record and model the PWR Raman intensity as the incident angle of light is scanned for thin polystyrene films coated on sapphire/gold substrates. PWR-Raman measurements of polystyrene films with thick- nesses of 276, 412, and $595 \mathrm{~nm}$ on $50 \mathrm{~nm}$ gold films were measured. Large Raman signals were recorded for thin polystyrene films down to a few hundred nanometers using $\mathrm{p}$ - and spolarized excitation; the Raman signal and polystyrene thickness can be well modeled by electric field calculations.

\section{EXPERIMENTAL}

\section{Sample Preparation}

Gold films were prepared on 25.4 mm diameter sapphire discs (Meller Optics, Providence, RI) by deposition of $2 \mathrm{~nm}$ Ti followed by $49 \pm 1 \mathrm{~nm}$ of gold at GWC Technologies Inc., Madison, WI. Prior to coating, the gold film was cleaned in ethanol 
and dried with a stream of N2 gas. A 4.6, 6, or $8 \%(w / v)$ polystyrene (Sigma-Aldrich, St. Louis, MO) solution was prepared in toluene (Fisher Scientific, Waltham, MA), and 200 $\mu \mathrm{L}$ of the polystyrene solution was spin-coated on the gold-coated sapphire disk at 3000 rpm for 1 min using a KW-4A spin-coater (Chemat Technology, Inc. Northridge, CA). The sample was dried overnight at room temperature to make certain the solvent was completely evaporated. Gold and polymer film thicknesses were measured using a F20 series film measurement system in transmission mode (Filmetrics, San Diego, CA).

\section{Raman Measurements}

A scanning angle Raman microscope with $0.05^{\circ}$ incident angle resolution was used to simultaneously collect Raman and reflectivity spectra.4 Raman data were collected with angle increments ranging from $0.05^{\circ}$ near the PWR angle to $0.20^{\circ}$ far away from the PWR angle. Incident excitation was from a $785 \mathrm{~nm}$ laser with $210 \mathrm{~mW}$ measured at the sample. The laser power had a $0.4 \%$ relative uncertainty for all Raman measurements, which means the laser power varied by no more than $\sim 1 \mathrm{~mW}$ from sample to sample. A $25.4 \mathrm{~mm}$ diameter sapphire prism was used for the total internal reflection element, and a $10 \times$ magnification objective (Nikon, numerical aperture 0.3 ) was used to collect the Raman scatter. Immersion oil (Cargille Laboratories, Cedar Groove, NJ, $n=1.7800$ ) was used to ensure optical contact between the prism and substrate. A half-wave plate in the excitation path was used to control the polarization of the incident light at the sample interface. The polarization at the sample was $99+\%$ pure. All spectra were acquired with 1 min acquisitions, and three replicate measurements were 
obtained for each sample by taking consecutive scans through the entire incident angle range.

Depolarization Ratio Measurements

$20 \%$ (w/v) polystyrene was dissolved in carbon tetrachloride (Sigma Aldrich, St. Louis, MO). A near-infrared polarizer was placed in the illumination path, and a second polarizer was placed between the microscope and spectrometer. A scrambler was added between the second polarizer and spectrometer. Raman spectra were collected for $30 \mathrm{~s}$ with the collection polarizer set to collect TE or TM polarized Raman scatter.

\section{Data Analysis}

All data analysis was performed using the software IGOR Pro 6.1. Raman peak areas were measured by fitting them to Gaussian curves with the "Multipeak fitting 2" algorithm. Signal-to-noise ratios $(\mathrm{S} / \mathrm{N})$ were calculated as the maximum of the $1001 \mathrm{~cm}^{-1}$ peak intensity after background subtraction divided by the standard deviation of the noise measured from 920 to $950 \mathrm{~cm}^{-1}$ in a region of the spectrum where no analyte peaks were present. Fresnel calculations were used to model the reflected light intensity. ${ }^{31}$ The Fresnel calculations account for the $0.5^{\circ}$ incident angle spread that results from focusing within the prism.

\section{Mean-Square Electric Field Calculations}

3-D finite difference-time-domain (FDTD)-based simulations were used to calculate the MSEF at varying incident angles (EM Explorer, San Francisco, CA). A Yee cell size of $5 \mathrm{~nm}$ was used for all FDTD simulations, and the calculations account for 
multiple reflections within the polymer layer. The interface had a sapphire prism $\left(\eta_{\mathrm{o}}\right.$ $\left.1.761, \eta_{\mathrm{e}} 1.753\right), 50 \mathrm{~nm}$ gold film $(\eta 0.143+4.799 \mathrm{i})$, and a polystyrene layer of varying

thickness ( $\eta$ 1.578), and the bulk medium was air. ${ }^{32,33}$ The angular resolution used for the calculations was $0.05^{\circ}$ near the PWR angle and $0.5^{\circ}$ elsewhere.

\section{RESULTS AND DISCUSSION}

\section{Calculated Reflectivity and Predicted Raman Peak Areas for a Sapphire/50 nm} Au/Polystyrene/Air Interface

The purpose of this study is to measure and model the PWR Raman signal for polystyrene waveguides of varying thickness. Determining the polymer thickness and chemical composition is possible by analyzing the PWR-Raman spectra as a function of incident angle. Figure 1 shows the calculated reflected light intensity (Figure 1A,C) and MSEF integrated over the polymer thickness (Figure 1B,D) at a sapphire/50 nm gold film interface containing a $276 \mathrm{~nm}$ (solid black), $412 \mathrm{~nm}$ (solid gray), or $595 \mathrm{~nm}$ (dotted black) polystyrene film. Using p-polarized excitation, $\mathrm{MSEF}_{\mathrm{Z}+\mathrm{X}}$ is generated (Figure 1B), while s-polarized excitation generates $\mathrm{MSEF}_{\mathrm{Y}}$ (Figure 1D). The coordinate system used in this work is shown in Figure 1.

For p-polarized excitation, the reflected light intensity is attenuated at some angles within the angle range of $34.5^{\circ}-60^{\circ}$ for all three interfaces. In this range, the angle where the attenuation of the reflected light intensity occurs is commonly referred to at the PWR angle. ${ }^{34}$ The PWR angle undergoes a shift from $34.51^{\circ}$ to $40.52^{\circ}$ when the film thickness 
is increased from 276 to $412 \mathrm{~nm}$. The reflectivity curve for the $595 \mathrm{~nm}$ polystyrene film contains two waveguiding modes $(\mathrm{m}=0$ and $\mathrm{m}=1)$ using $\mathrm{p}$-polarized excitation, giving rise to PWR angles of $34.48^{\circ}$ and $50.77^{\circ}$, respectively. The PWR angle generated with spolarized light is not the same as the PWR angle generated with p-polarized light. Although the associated angles and magnitudes are different, qualitatively, the same trends are expected in the reflectivity curves using s-polarized excitation. The shift in the PWR angle is sensitive to small changes in the polystyrene thickness. For example, the PWR angle for a $600 \mathrm{~nm}$ polystyrene film will undergo a $0.37^{\circ}$ (p-polarization) and a $0.15^{\circ}$ (s-polarization) shift when the thickness is increased to $610 \mathrm{~nm}$.

For polystyrene films and using a $785 \mathrm{~nm}$ wavelength laser, the cutoff thickness for PWR Raman spectroscopy using a prism/gold/polystyrene/air interface with p- and spolarized excitation is approximately 260 and $140 \mathrm{~nm}$, respectively. For films thinner than this, PWR Raman spectroscopy using a prism/gold/silica/polystyrene/bulk medium interface may be utilized. ${ }^{35}$ In theory polystyrene films of several micrometers can be measured with PWR Raman spectroscopy. A $5 \mu \mathrm{m}$ film will produce 15 distinct waveguide modes with $\mathrm{p}$ - or s-polarized excitation over an angle range of $35^{\circ}-65^{\circ}$. In practice, films of several micrometers thickness present an experimental challenge due to the required angular resolution. The fwhm for the PWR peaks corresponding to polymer thickness below $\sim 600 \mathrm{~nm}$ range from $0.04^{\circ}$ to $0.5^{\circ}$; the PWR peaks with s- polarized excitation are generally narrower than those with p- polarized excitation. Experimental measurements should have an angular resolution equal to or smaller than the fwhm. 
The sharp attenuation of the reflected light at the PWR angle is a near mirror reflection of the corresponding MSEF curves (Figure 1B,D), which is expected to model the Raman scatter. The $276 \mathrm{~nm}$ polystyrene film is at the cusp of the polymer thickness required to generate a PWR peak using p-polarized incident light and is associated with the lowest calculated MSEF (maximum integrated $\mathrm{MSEF}_{\mathrm{Z}+\mathrm{X}} 38.1$ ). The 412 and $595 \mathrm{~nm}$ polystyrene films, on the other hand, exhibit distinct PWR peaks. In contrast to ppolarized excitation, even the thinnest film is expected to exhibit significant Raman signals (maximum integrated $\mathrm{MSEF}_{\mathrm{Y}}$ up to 17700) using s-polarized excitation.

At an angle greater than $60^{\circ}$ there is a broad attenuation dip in the reflectivity curve using p polarized excitation. The angle where this dip occurs is referred to as the SPR angle and is the angle where the maximum Raman scatter is predicted in SPRRaman spectroscopy. The MSEF enhancement is similar at the SPR angle regardless of whether the interface supports waveguide modes (Table 1). On the other hand, the MSEF enhancement is greater at the PWR angle than the SPR angle, and s-polarized light generates a $\mathrm{MSEF}_{\mathrm{Y}}$ component at the waveguide interface.

Compared to an interface without the gold film, the calculated MSEF at the gold film is approximately $10-50 \times$ higher. Since the MSEF is proportional to the expected Raman scatter generated at the interface, it is proposed that the PWR Raman measurements will generate more Raman scatter when the same polymer thickness is considered. The expected, large PWR Raman signal means one or several second acquisition times should generate significant Raman scatter, which decreases the total analysis time per sample without compromising the spectral signal-to-noise ratio. 


\section{Experimental Reflectivity Curves and Determination of Polystyrene Thickness}

Reflectivity curves collected with p-polarized incident light are shown in Figure 2A, B, C for three thicknesses of polystyrene. Only the films fabricated using 6 or $8 \%$ $(\mathrm{w} / \mathrm{v})$ polystyrene generated a PWR peak, while the $4.6 \%(\mathrm{w} / \mathrm{v})$ film produced only an SPR peak. The reflectivity curve of an $8 \%(w / v)$ polystyrene film using s-polarized incident light is shown in Figure 2D. The polystyrene film thickness was determined by modeling the location of the PWR or SPR angle(s) using Fresnel calculations. ${ }^{31}$ While holding all other parameter constant, the thickness of the polystyrene layer was varied until the best fit was obtained (Figure 2: A, $276 \mathrm{~nm}$; B, $412 \mathrm{~nm}$; and C, D, $595 \mathrm{~nm}$ ). For the $595 \mathrm{~nm}$ polystyrene film, the thickness that generated the best fit to the PWR peaks did not fit the SPR peak as well. A small increase in the imaginary component of gold's index of refraction will shift the calculated SPR peak to the left, with minimal perturbations to the PWR peak. This suggests there is heterogeneity in the gold films on which the polymer was deposited, which is reasonable since they were fabricated in several batches. For the $595 \mathrm{~nm}$ polystyrene film using s-polarized excitation, the attenuation of the reflected light corresponding to the $\mathrm{m}=1$ guided mode at $41.80^{\circ}$ is experimentally measured, but the predicted $\mathrm{m}=0$ mode at $57.57^{\circ}$ is not. The fwhm of the PWR reflectivity peaks are calculated to be roughly $6 \times$ narrower than the SPR peaks. The lack of a measured PWR peak at $57.57^{\circ}$ is attributed to the narrowness of the PWR peak (fwhm $0.04^{\circ}$ ) and the instrument's angular resolution of $0.05^{\circ}$. This is also the reason the experimental reflectivity minima is less than predicted in several instances. 
The polymer thicknesses obtained from the modeled reflectivity data agree with a calibration curve generated using optical interferometry (data not shown). The difference between the scanning angle Raman and optical interferometry measurements is associated with an average difference of $2.6 \%$ for all three films. Potential errors involved in the thickness measurement include the instrument's incident angle calibration, the finite angular resolution of the instrument, and uncertainties in the gold film thickness or indices of refraction of any of the interfacial layers.

PWR-Raman Spectra of Polystyrene Films

Raman spectra are shown in Figure 3 for the same samples used to simultaneously generate the reflectivity data in Figure 2. Assignments for the most intense peaks are shown in Table 2. The Raman spectra were collected at an incident angle of $68.40^{\circ}(\mathrm{p}$, $276 \mathrm{~nm}$ polystyrene), $40.52^{\circ}$ (p, $412 \mathrm{~nm}$ polystyrene), $50.77^{\circ}$ (p, $595 \mathrm{~nm}$ polystyrene), or $41.80^{\circ}$ (s, $595 \mathrm{~nm}$ polystyrene), which correspond to the incident angles that generated the greatest Raman scatter. For the $276 \mathrm{~nm}$ film, the highest Raman scatter was collected at the SPR angle, which is expected since no PWR peak was measured for this film. The higher $1003 \mathrm{~cm}^{-1}$ peak intensity for s-polarized excitation compared to p-polarized excitation quantitatively agrees with the calculated MSEF from Figure 1 when the magnitude of the reflected light intensity is considered.

The PWR Raman spectrum's S/N ratio for the $412 \mathrm{~nm}$ polystyrene film is 602 (Figure 3B) using a $60 \mathrm{~s}$ acquisition time, which is significantly better than the $\mathrm{S} / \mathrm{N}$ ratio of 28 for a $580 \mathrm{~nm}$ film at the sapphire interface without a gold film (data not shown). The gold film enables the acquisition time to be reduced to a couple of seconds per 
spectrum to achieve the same $\mathrm{S} / \mathrm{N}$ ratio as a $60 \mathrm{~s}$ acquisition for a similar film thickness deposited on sapphire. It should also be stated that none of the polystyrene waveguides produced detectable Raman scatter using a $180^{\circ}$ backscatter geometry with the same instrument. The insets in Figure 3C,D show the spectra normalized to the most intense polystyrene peak $\left(1003 \mathrm{~cm}^{-1}\right)$ to compare the relative peak intensities using different excitation polarizations. PWR-Raman measurements of polystyrene show certain bands produce more Raman scatter with p-polarized excitation compared to s-polarized excitation when the spectra are normalized to the $1003 \mathrm{~cm}^{-1}$ polarized peak. Table 2 lists the ratio of the PWR Raman peak intensity for s/p-polarized excitation and the $595 \mathrm{~nm}$ film. Also shown in Table 2 are the depolarization ratios measured for a solution of polystyrene with the $180^{\circ}$ backscattering geometry. The polarized bands produced a s $/ \mathrm{p}$ peak intensity ratio greater than 0.8 when comparing spectra normalized to the $1003 \mathrm{~cm}^{-1}$ peak. The depolarized bands produce ratios from 0.5 to 0.6 . The origin for the differences in the scanning angle Raman spectra using orthogonal excitation has been previously discussed. ${ }^{12}$

\section{Measured Raman Peak Areas and Calculated MSEF}

The Raman peak areas of the polystyrene $1003 \mathrm{~cm}^{-1}$ band are plotted as a function of incident angle in Figure 4 for the same samples discussed above. The Raman peak areas fit to the calculated integrated MSEF using the appropriate polymer thickness. The maximum Raman peak area always corresponds to the angle producing the greatest attenuation of the reflected light intensity; thus, the reflected light intensity can be used to identify the angle producing the largest Raman signal. As expected, this is in good 
agreement with previous SPR studies that have demonstrated that reflectivity data can be used to predict the collected Raman intensity. ${ }^{12}$ The slight differences in the predicted Raman peak area and the measured Raman peak area can be attributed to the same causes discussed above for differences in the reflectivity data (i.e., angle resolution, angle spread, etc.).

The plot of the MSEF as a function of distance from the gold/polystyrene interface (Figure 5) at the PWR angle indicates the collected Raman scatter is not always uniformly generated throughout the polymer film. The radiative or "leaky" waveguide mode maxima are the result of interference between courter-propagating reflections. ${ }^{36}$ For thinner polystyrene films and p-polarized excitation only a single leaky mode exists, but for all films with s-polarization and the $595 \mathrm{~nm}$ polystyrene film with p-polarized excitation two leaky waveguide modes exist. Both the polymer thickness and the excitation polarization will affect the spatial distribution of the Raman signal within the polymer film. For the 276 and $412 \mathrm{~nm}$ polystyrene film, p- polarized excitation will produce Raman intensities nearly uniformly from the entire polymer film at the PWR angle. With thicker films using p-polarized excitation and all thicknesses using spolarized excitation, the spatial distribution of the MSEF is more complex. For example, the strongest Raman intensity will be generated near the center of the polymer films using s-polarized excitation with the $412 \mathrm{~nm}$ film at the PWR angle indicated in the figure legend. For films that contain more than one polymer, the MSEF versus distance plots can be used to aid in the interpretation of polymer structure or order. 


\section{CONCULSIONS}

This proof of principle study shows scanning angle plasmon waveguide resonance Raman spectroscopy can measure thin polymer films with predicable signal enhancements, to obtain simultaneous chemical content and accurate polymer film thicknesses. A polystyrene/air interface was used in this study to ensure that the entire Raman signal was generated from polystyrene and no polymer swelling or contamination occurred. However, other polymer films that are of high optical quality, transparent, and meet the thickness requirements of an optical waveguide could be measured, as could water or organic interfaces. Overall, the method demonstrates that PWR-Raman spectroscopy utilizing a simple plasmonic supporting system allows for optimal experimental modeling of thin films.

\section{ACKNOWLEDGEMENTS}

This research is supported by the U.S. Department of Energy, Office of Basic Energy Sciences, Division of Chemical Sciences, Geosciences, and Biosciences through the Ames Laboratory. The Ames Laboratory is operated for the U.S. Department of Energy by Iowa State University under Contract No. DE-AC02-07CH11358. The authors thank the Iowa State University Microelectronics Research Center for use of their Filmetrics instrument. 


\section{REFERENCES}

(1) Ikeshoji, T.; Ono, Y.; Mizuno, T. Appl. Opt. 1973, 12, 2236.

(2) Woods, D. A.; Bain, C. D. Analyst 2012, 137, 35.

(3) Tisinger, L. G.; Sommer, A. J. Microsc. Microanal. 2004, 10, 1318.

(4) McKee, K. J.; Smith, E. A. Rev. Sci. Instrum. 2010, 81, 043106/1.

(5) Hoelzer, W.; Schroeter, O.; Richter, A. J. Mol. Struct. 1990, 217, 253.

(6) Woods, D. A.; Petkov, J.; Bain, C. D. J. Phys. Chem. B 2011, 115, 7341.

(7) Woods, D. A.; Petkov, J.; Bain, C. D. J. Phys. Chem. B 2011, 115, 7353.

(8) Greene, P. R.; Bain, C. D. Colloids Surf., B 2005, 45, 174.

(9) Bolduc, O. R.; Masson, J. F. Anal. Chem. 2011, 83, 8057.

(10) Liu, Y.; Xu, S. P.; Xuan, X. Y.; Zhao, B.; Xu, W. Q. J. Phys. Chem. Lett. 2011, 2, 2218.

(11) Corn, R. M.; Philpott, M. R. J. Chem. Phys. 1984, 80, 5245.

(12) McKee, K. J.; Meyer, M. W.; Smith, E. A. Anal. Chem. 2012, 84, 4300.

(13) Harsanyi, G. Sens. Rev. 2000, 20, 98.

(14) Fina, L. J. Appl. Spectrosc. Rev. 1994, 29, 309.

(15) Yang, P.; Meng, X. F.; Zhang, Z. Y.; Jing, B. X.; Yuan, J.; Yang, W. T. Anal. Chem. 2005, 77, 1068 . 
(16) Bohn, P. W. Annu. Rev. Mater. Sci. 1997, 27, 469.

(17) Ishizaki, F.; Kim, M. Jpn. J. Appl. Phys. 2008, 47, 1621.

(18) Fontaine, N. H.; Furtak, T. E. Phys. Rev. B 1998, 57, 3807.

(19) Zimba, C. G.; Hallmark, V. M.; Turrell, S.; Swalen, J. D.; Rabolt, J. F. J. Phys. Chem. 1990, 94, 939.

(20) Fontaine, N. H.; Furtak, T. E. J. Opt. Soc. Am. B 1997, 14, 3342.

(21) Ives, J. T.; Reichert, W. M. J. Appl. Polym. Sci. 1988, 36, 429.

(22) Kivioja, A. O.; Jaaskelainen, A. S.; Ahtee, V.; Vuorinen, T. Vib. Spectrosc. 2012, $61,1$.

(23) Chabay, I. Anal. Chem. 1982, 54, A071.

(24) Im, W. J.; Kim, B. B.; Byun, J. Y.; Kim, H. M.; Kim, M.-G.; Shin, Y.-B. Sens. Actuators, B 2012, 173, 288.

(25) Abbas, A.; Linman, M. J.; Cheng, Q. Sens. Actuators, B 2011, 156, 169.

(26) Zourob, M.; Mohr, S.; Brown, B. J. T.; Fielden, P. R.; McDonnell, M. B.; Goddard, N. J. Anal. Chem. 2005, 77, 232.

(27) Kanger, J. S.; Otto, C. Appl. Spectrosc. 2003, 57, 1487.

(28) Zimba, C. G.; Rabolt, J. F. Thin Solid Films 1991, 206, 388.

(29) Bradshaw, J. T.; Mendes, S. B.; Saavedra, S. S. Anal. Chem. 2005, 77, 28 a. 
(30) Hruby, V. J.; Alves, I.; Cowell, S.; Salamon, Z.; Tollin, G. Life Sci. 2010, 86, 569.

(31) Corn, R. M.; http://corninfor.ps.uci.edu/calculations.html.

(32) Kasarova, S. N.; Sultanova, N. G.; Ivanov, C. D.; Nikolov, I. D. Opt. Mater. 2007, $29,1481$.

(33) Bass, M.; DeCusatis, C.; Enoch, J. M.; Lakshminarayanan, V.; Li, G.; MacDonald, C.; Mahajan, V. N.; Van Stryland, E. Handbook of Optics, 3rd ed.; McGraw-Hill: New York, 2009; Vol. IV.

(34) Salamon, Z.; Tollin, G. Spectrosc.: Int. J. 2001, 15, 161.

(35) McKee, K. J.; Meyer, M. W.; Smith, E. A. Anal. Chem. 2012, 84, 9049.

(36) Zia, R.; Selker, M. D.; Brongersma, M. L. Phys. Rev. B 2005, 71, 165431.

(37) Loader, E. J. J. Catal. 1971, 22, 41.

(38) Palm, A. J. Phys. Chem. 1951, 55, 1320. 
Table 1. Comparison of surface plasmon resonance and plasmon waveguide resonance parameters for thin polystyrene films at the sapphire/50 $\mathrm{nm}$ gold/polymer interface.

\begin{tabular}{|c|c|c|c|c|}
\hline Platform & $\begin{array}{c}\text { SPR/PWR } \\
\text { angle } \\
\text { (degrees) }\end{array}$ & $\begin{array}{c}\text { FWHM } \\
\text { (degrees) }\end{array}$ & $\begin{array}{c}\text { MSEF }_{\text {Ex }+ \text { Ez }} \\
\text { Integrated } \\
\text { Over Polymer } \\
\text { Film }\end{array}$ & $\begin{array}{c}\text { MSEF }_{\text {Ey }} \\
\text { Integrated } \\
\text { Over Polymer } \\
\text { Film }\end{array}$ \\
\hline $\begin{array}{c}\text { Non-waveguide }(100 \mathrm{~nm}) \\
\text { SPR }\end{array}$ & 50.69 & 2.24 & 786 & 0.0 \\
\hline $\begin{array}{c}\text { Waveguide (412 nm) } \\
\text { SPR }\end{array}$ & 70.67 & 2.76 & 839 & 0.0 \\
\hline PWR (p) & 40.52 & 0.48 & 3776 & 0.0 \\
\hline PWR (s) & 52.95 & 0.12 & 0.0 & 9651 \\
\hline
\end{tabular}


Table 2. Peak location, assignment and relative intensity ratio for s/p-polarized excitation for scanning angle Raman spectra of polystyrene; measured depolarization ratios for a solution of polystyrene.

\begin{tabular}{|c|c|c|c|}
\hline Peak $\left(\mathrm{cm}^{-1}\right)$ & $\begin{array}{c}\text { Assignment } \\
\text { (Wilson Notation) } \\
33\end{array}$ & $\begin{array}{c}\text { Relative } \\
\text { Intensity } \\
(\mathrm{S}) /(\mathrm{P}): \text { PWR } \\
\text { Raman } \\
\text { Spectroscopy }\end{array}$ & $\begin{array}{c}\text { Solution } \\
\text { Depolarization } \\
\text { Ratio: Normal } \\
\text { illumination } \\
\text { Raman } \\
\text { Spectroscopy }\end{array}$ \\
\hline 624 & $\mathrm{v}_{6 \mathrm{~b}}$ & 0.5 & 1.08 \\
\hline 760 & & 0.8 & ca. 0 \\
\hline 795 & $\mathrm{v}_{11}$ & 1 & ca. 0 \\
\hline 843 & $\mathrm{v}_{10 \mathrm{a} / 17 \mathrm{~b}}$ & 0.5 & \\
\hline 906 & vinyl & 0.5 & \\
\hline 1003 & $\mathrm{v}_{1}$ & 1 & 0.06 \\
\hline 1031 & $\mathrm{v}_{18 \mathrm{a}}$ & 0.9 & ca. 0 \\
\hline 1073 & & 0.5 & \\
\hline 1097 & & 0.6 & \\
\hline 1156 & $\mathrm{~V}_{9 b}$ & 0.5 & 0.84 \\
\hline 1184 & $\mathrm{v}_{9 \mathrm{a}}$ & 0.5 & 0.65 \\
\hline 1201 & $\mathrm{v}_{7 \mathrm{a}}$ & 0.8 & ca. 0 \\
\hline 1304 & vinyl & 0.6 & \\
\hline 1330 & $\mathrm{v}_{14}$ & 0.5 & \\
\hline 1346 & $v_{3}$ & 0.5 & \\
\hline 1368 & vinyl & 0.5 & \\
\hline 1451 & $\mathrm{v}_{19 \mathrm{~b} / \mathrm{a}}$ & 0.5 & \\
\hline 1584 & $\mathrm{v}_{8 \mathrm{~b}}$ & 0.5 & 1.22 \\
\hline 1602 & $\mathrm{~V}_{8 \mathrm{a}}$ & 0.5 & 0.91 \\
\hline
\end{tabular}




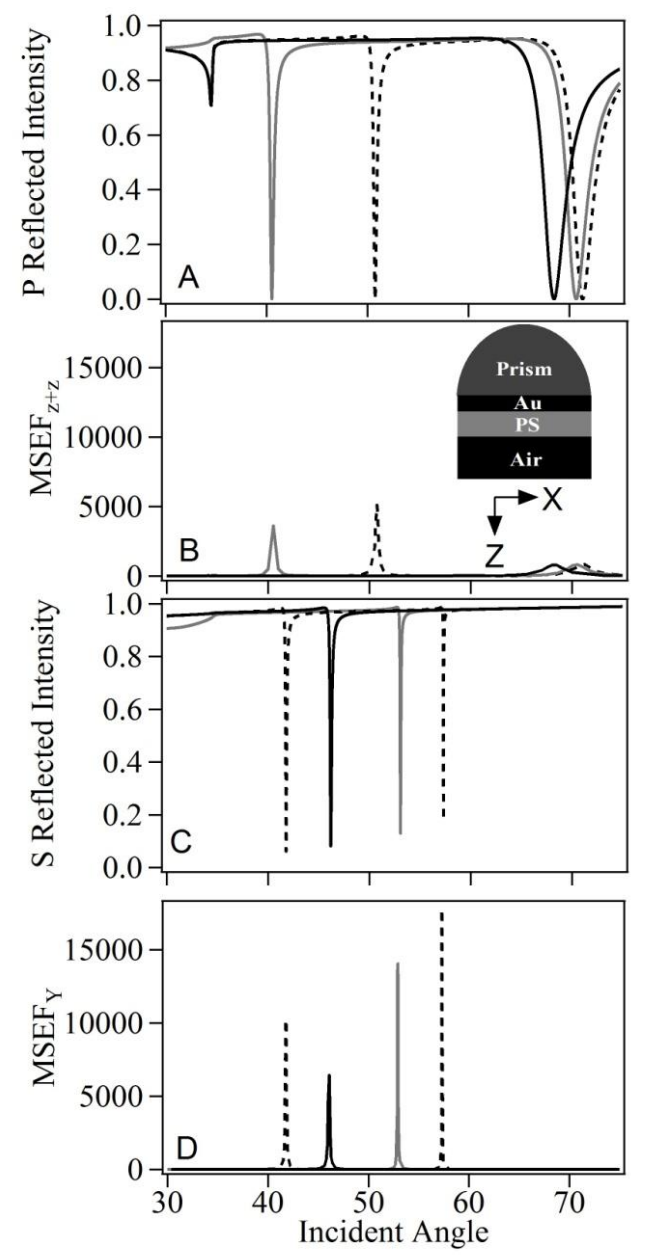

Figure 1. Calculated reflectivity (A and C) and mean square electric field integrated over the polymer stack (MSEF, B and D) for a sapphire prism/50 nm gold/polystyrene (PS)/air interface. The thickness of the polystyrene film is $276 \mathrm{~nm}$ (solid black), $412 \mathrm{~nm}$ (solid gray), or $595 \mathrm{~nm}$ (dotted black). The reflectivity calculations used a 0.5 degree incident angle spread in the excitation light. P-polarized incident light (A, B) generates a MSEF in the $\mathrm{Z}$ and $\mathrm{X}$ directions, and s-polarized incident light $(\mathrm{C}, \mathrm{D})$ generates a MSEF in the Y direction. The inset schematic shows the coordinate system used in this work; the $\mathrm{Y}$ axis points towards the reader. 


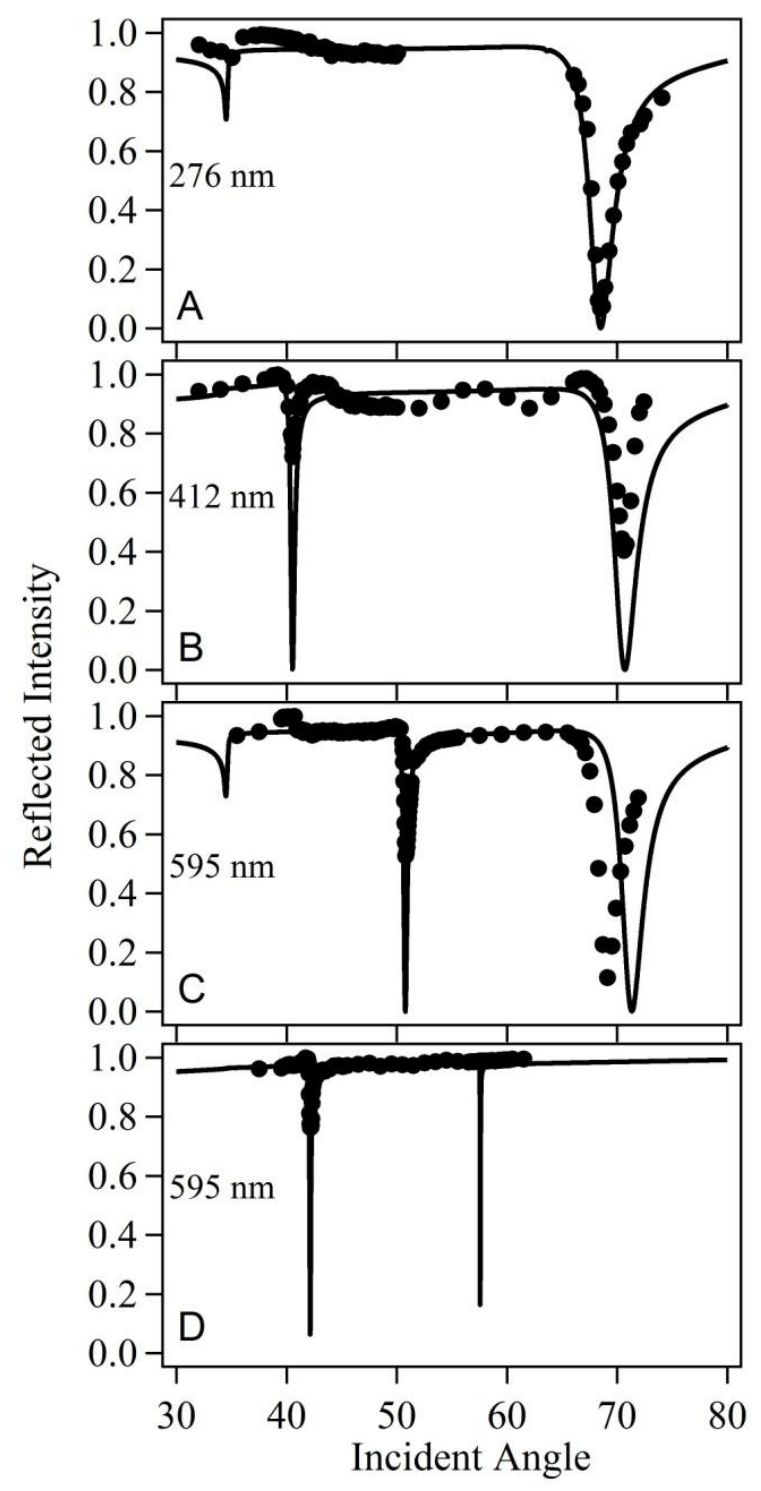

Figure 2. Reflectivity spectra (solid symbols) from a sapphire prism/50 nm gold/polystyrene (PS)/air interface: (A) 276 nm PS; (B) 412 nm PS; (C) 595 nm PS collected with p-polarized incident excitation. (D) Sapphire prism/50 nm gold $/ 595 \mathrm{~nm}$ polystyrene (PS)/air interface collected with s-polarized incident excitation. The solid lines are calculated reflectivities for the same interfaces. 


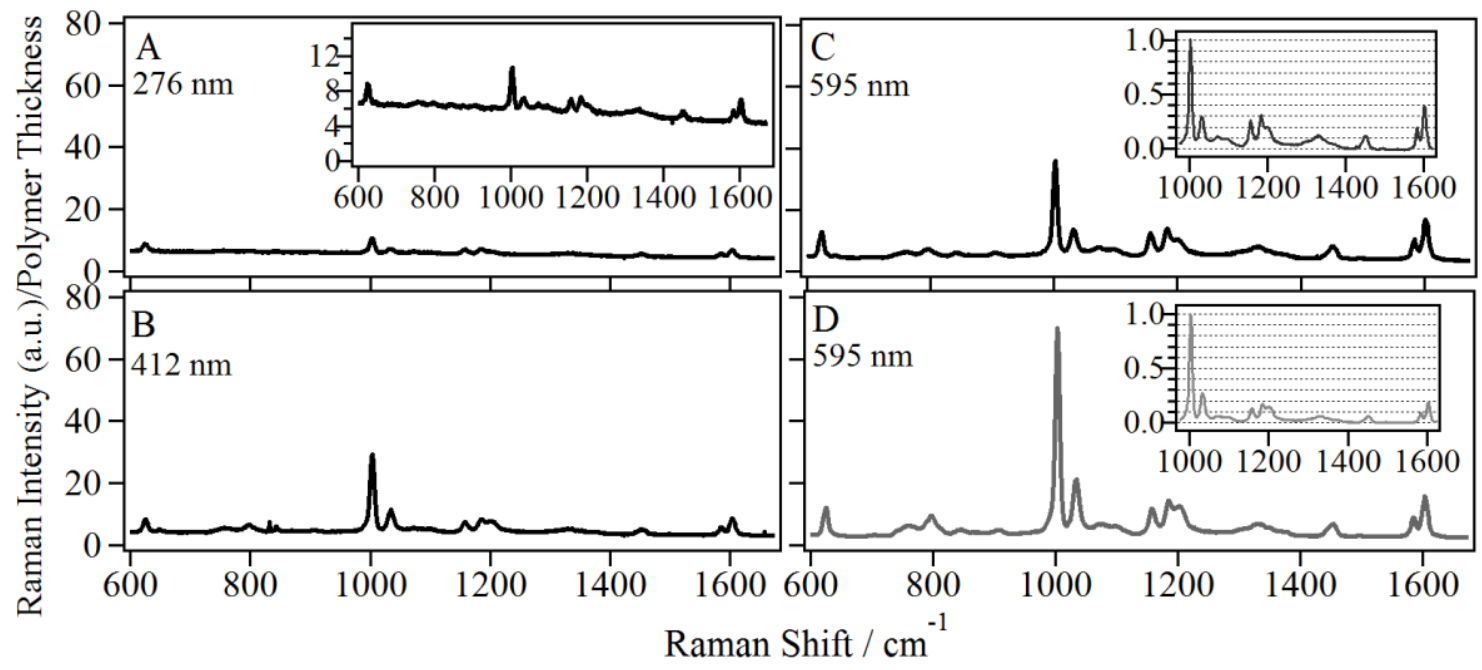

Figure 3. Raman spectra of a sapphire prism/50 nm gold/polystyrene (PS)/air interface: (A) $276 \mathrm{~nm}$ PS; (B) $412 \mathrm{~nm}$ PS; (C) $595 \mathrm{~nm}$ PS collected with p-polarized incident excitation at 68.40, 40.52, 50.77 degree incident angle, respectively. (D) Sapphire prism/50 nm gold/595 nm polystyrene (PS)/air interface collected with s-polarized incident excitation at 41.80 degree incident angle. The inset in (A) shows the same spectrum at a smaller scale. The inset in (C) and (D) show the normalized spectra so that relative peak intensities can be compared. All spectra have been divided by their respective film thickness. The spectra do not account for the slight elongation of the beam size for larger incident angles (for example, $242 \mu \mathrm{m}$ x 179 um at 40 and 271 x 177 $\mu \mathrm{m}$ at 68 degrees). 

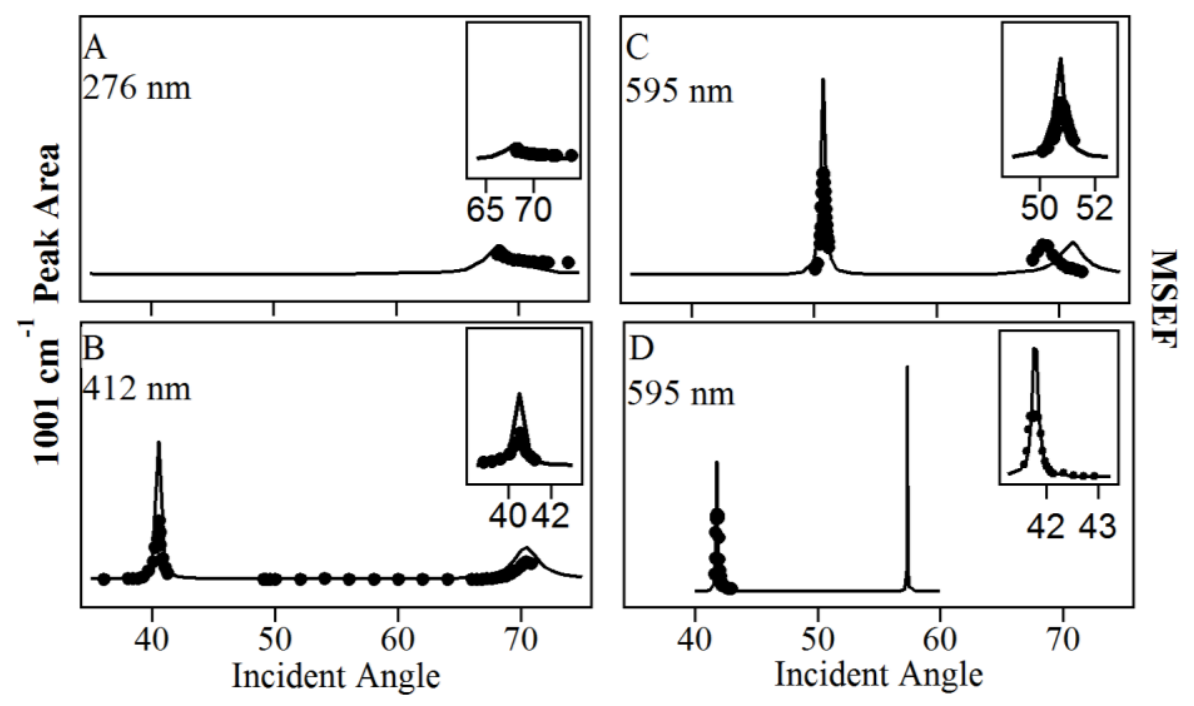

Figure 4. Raman $1003 \mathrm{~cm}-1$ peak areas (black spheres) as a function of incident angle for a sapphire prism/50 nm gold/polystyrene (PS)/air interface: (A) $276 \mathrm{~nm}$ PS; (B) 412 nm PS; (C) 595 nm PS collected with p-polarized incident excitation. (D) Sapphire prism/50 nm gold/595 nm polystyrene (PS)/air interface collected with s-polarized incident excitation. The solid black line is the calculated $\operatorname{MSEF}\left(\mathrm{A}, \mathrm{B}, \mathrm{C}: \mathrm{MSEF}_{\mathrm{Z}+\mathrm{X}}\right.$ and D: $\mathrm{MSEF}_{\mathrm{Y}}$ ) integrated over the entire polymer thickness. Example MSEF plots at select incident angles are plotted in Figure 5. 

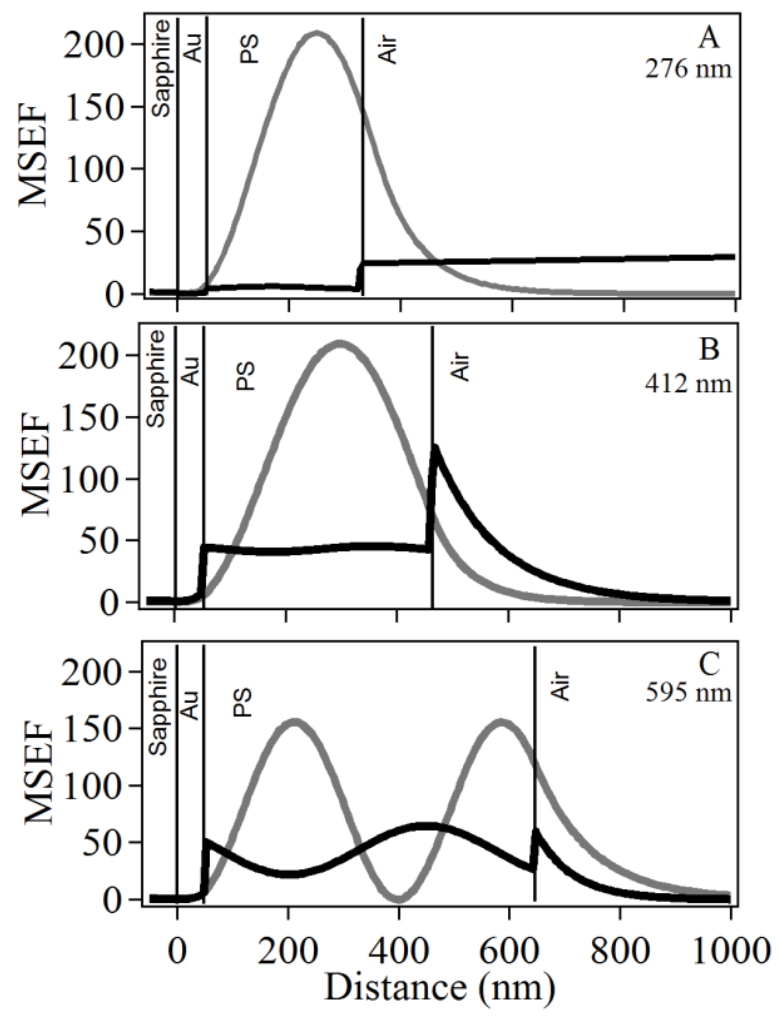

Figure 5. Calculated MSEF as a function of the distance from the interface for $\mathrm{p}$ polarized (black) and s-polarized (gray) incident excitation and a sapphire prism/50 nm gold/ polystyrene (PS)/air stack: (A) $276 \mathrm{~nm}$ PS at 34.51 (p-polarized excitation, $\mathrm{MSEF}_{\mathrm{Z}+\mathrm{X}}$ ) and 46.20 (s-polarized excitation, $\mathrm{MSEF}_{\mathrm{Y}}$ ) degrees; (B) 412-nm PS at 40.52 (p) and 53.07 (s); (C) $595 \mathrm{~nm}$ PS at 50.77 (p) and 41.80 (s). The location of each phase in the stack is shown with a solid vertical line. 


\title{
CHAPTER 4: SCANNING ANGLE TOTAL INTERNAL REFLECTION RAMAN SPECTROSCOPY OF THIN POLYMER FILMS
}

A paper published in MRS Online Proceedings Library. 2013, 1552, doi:10.1557/opl.2013.133

Matthew W. Meyer and Emily A. Smith

\begin{abstract}
Ames Laboratory, U. S. Department of Energy, Ames, Iowa 50011-3111, and Department of
\end{abstract}

Chemistry, Iowa State University, Ames, Iowa 50011-3111

\begin{abstract}
Plasmon waveguide resonance (PWR) Raman spectroscopy provides chemical content information with interface or thin film selectivity. Near the plasmon waveguide interface, large increases in the interfacial optical energy density are generated at incident angles where plasmon waveguide resonances are excited. When a polymer of sufficient thickness is deposited on a gold film, the interface acts as a plasmon waveguide and large
\end{abstract}


enhancements in the Raman signal can be achieved. This paper presents calculations to show how polymer thickness and excitation wavelength are predicted to influence PWR Raman spectroscopy measurements. The results show the optical energy density (OED) integrated over the entire polymer film using $785 \mathrm{~nm}$ excitation are $1.7 \times(400 \mathrm{~nm}$ film $)$, $2.17 \times(500 \mathrm{~nm}$ film $), 2.48 \times(600 \mathrm{~nm}$ film $), 3.08 \times(700 \mathrm{~nm}$ film $)$ and $3.62 \times(800 \mathrm{~nm}$ film $)$ higher compared to a $300 \mathrm{~nm}$ film. Accounting for the integrated OED and frequency to the fourth power dependence of the Raman scatter, a $532 \mathrm{~nm}$ excitation wavelength is predicted to generate the largest PWR Raman signal at the polymer waveguide interface. This work develops a foundation for chemical measurements of numerous devices, such as solar energy capturing devices that utilize conducting metals coated with thin polymer films.

\section{INTRODUCTION}

Plasmon waveguide resonance (PWR) Raman spectroscopy is a label free spectroscopic method for interfacial analysis with chemical specificity [1-2]. In a standard PWR Raman spectroscopy experiment the illuminating light is directed onto a prism/metal/dielectric/bulk interface at a specific incident angle. Raman scatter and reflectivity data are collected simultaneously for each incident angle above the critical angle for total internal reflection [3]. Conventional Raman spectroscopy, when the illuminating light is not under total internal reflection, suffers in studying interfaces and thin films due to a lack of interfacial selectivity and the small signals that result from reduced analysis volumes. Plasmon waveguides operate by coupling surface plasmon

modes in the metal to guided modes within the dielectric layer or waveguide [4]. Recent work has shown that the amplified electric field at a waveguide interface can be used for 
generating Raman spectra with large signal-to-noise ratios for solutions, polymer films and monolayers [1-2].

Two recently reported sample geometries suitable to measure PWR Raman spectra of thin polymer films are shown in Figure 1. The interface shown in Figure 1A is suitable for measuring polymer films down to a couple nanometers in thickness using $785 \mathrm{~nm}$ excitation. The interface contains a $600 \mathrm{~nm}$ silica waveguide layer with the polymer deposited on top of the silica. The electric field within the polymer layer (shaded green, Figure 1A) is responsible for producing Raman scatter. Dielectric materials other than silica could also be used provided the surface is smooth, the dielectric thickness is large enough to support guided waves and the dielectric provides a minimal spectral background.

An alternative sample geometry uses the polymer as the waveguiding layer when it is directly deposited on the gold film (Figure 1B). The required polymer thickness for a radiative waveguide with $785 \mathrm{~nm}$ excitation and polystyrene is approximately $250 \mathrm{~nm}$ (i.e., $\lambda / 2 n$, where $\lambda$ is the excitation wavelength and $n$ is the refractive index of the polymer). The electric field generated within the polymer film (shaded purple, Figure 1B) shows a more complex pattern compared to when a silica waveguide is used, and in both geometries the electric field can be greatly enhanced compared to an interface without the gold film. The Raman scatter generated within a polymer film can be $10 \times$ or more using PWR Raman spectroscopy in comparison to a polymer film deposited on a prism without a gold film [1]. In this paper we expand on our preliminary work using PWR Raman spectroscopy to measure polymer films. We discuss the effect of polymer film thickness and excitation wavelengths on the optical energy density (OED) 
distribution within polystyrene films to predict how these parameters will affect PWR Raman measurements. The presented data lay a foundation for the study of a variety of thin organic photovoltaic materials.

\section{EXPERIMENTAL}

A 3-D finite-difference-time-domain calculation (EM Explorer, San Francisco, CA) was used to generate the reflectivity and OED distribution data at the various interfaces as the polystyrene thicknesses or excitation wavelength was varied. The calculations used a Yee cell size of $5 \mathrm{~nm}, 1000$ cycles, p-polarized incident light and $0.05^{\circ}$ angle resolution. The angular resolution corresponds to that of a recently described home-built scanning angle Raman spectrometer [5]. The interface was illuminated with an incident plane wave of $785 \mathrm{~nm}$ wavelength except for the data shown in Figure 4 where the wavelength was set to $488,532,580,633,700,850,950$ or $1064 \mathrm{~nm}$. The integrated OED calculated across the entire thickness of the polymer film was determined by integrating the area under the OED curve using an algorithm in the program IGOR 6.0. Indices of refraction were 1.762 (sapphire p- polarization), $0.143+4.799 i$ (gold), 1.578 (polystyrene) and 1.000 (air).

\section{DISCUSSION}

The expected intensity of PWR Raman scatter is directly related to the OED integrated across the entire polymer thickness. Therefore, it is desirable to understand how the experimental factors affect the OED. The interface that was the focus of these 
studies consisted of the following layers: sapphire prism/50 nm gold/polystyrene/air. The prism refractive index is greater than that of the polystyrene film, and the semi-infinite air layer provides the benefit of only generating Raman scatter from the polystyrene film without swelling or a physical change to the polymer film during experimental measurements. The following sections describe how the polymer film thickness and the excitation wavelength affect the OED distribution throughout the interface for polystyrene films ranging from $275 \mathrm{~nm}$ to $800 \mathrm{~nm}$.

\section{Optical energy density distribution generated in polystyrene films}

Figure 2A shows the OED distribution generated throughout the interface at the PWR angle as the polystyrene film thickness increases from $300 \mathrm{~nm}$ to $800 \mathrm{~nm}$. The electric field generated within the polystyrene film is cosinusoidal, while the electric field generated within the air layer decays exponentially with a maximum value at the polystyrene/air interface. For the $300 \mathrm{~nm}$ polystyrene film (blue) a majority of the total OED for the interface is generated in the air layer. As the polystyrene thickness increases, the fraction of the total OED that is generated in the air layer decreases, while the fraction that is generated in the polymer layer increases. The $300 \mathrm{~nm}$ polystyrene film produces a $4.6 \times$ greater OED at the polystyrene/air interface than that of the $800 \mathrm{~nm}$ polystyrene film (orange). This trend is important to note because the OED in the polystyrene film will govern the measured Raman intensity, while the OED produced in the air layer is not beneficial for the purpose of measuring the polymer composition.

The OED integrated over the entire polymer film is shown in Figure $2 \mathrm{~B}$ for varying polystyrene thicknesses. The integrated OED compared to a $300 \mathrm{~nm}$ film are 
$1.7 \times, 2.17 \times, 2.48 \times, 3.08 \times$ and $3.62 \times$ higher for $400,500,600,700,800 \mathrm{~nm}$ films, respectively. The polystyrene thickness cutoff for a radiative waveguide with $785 \mathrm{~nm}$ excitation is approximately $250 \mathrm{~nm}$. However, films thicker than approximately $325 \mathrm{~nm}$ are required to generate a large OED within the polymer film when the incident angle is \pm $0.05^{\circ}$ of the PWR angle (i.e., the calculations had an angle resolution of 0.05 ).

Larger Raman signals are expected for thicker polystyrene films primarily because more material is being measured. When the calculated integrated OED is divided by the polymer thickness (Figure 2B inset), the amount of signal generated per nanometer of film is expected to be approximately the same for films thicker than approximately $325 \mathrm{~nm}$. Some of the small variation observed with films thicker than 325 $\mathrm{nm}$ is due to the limited angle resolution for the calculations, and the extremely narrow PWR peaks that are calculated for these interfaces. The full width half maximum for the PWR peaks is close to or smaller than the angle resolution in some cases. A higher angular resolution would increase computation time, and the angular resolution that was chosen for the calculations matched an experimental set-up recently demonstrated for PWR Raman spectroscopy, as noted above.

\section{PWR angle shifts as a function of polystyrene thickness}

The PWR angle is identified as the angle producing the maximum attenuation of the reflected light intensity as the incident angle is scanned, and its location is dependent on the polymer film thickness and other optical properties of the interfacial layers. The maximum Raman scatter is generated at the PWR angle. For this reason PWR Raman spectroscopy can determine the polymer film thickness by monitoring the position of the 
PWR angle. Figure 3 shows the calculated PWR angle for interfaces containing 275 to $800 \mathrm{~nm}$ thick polystyrene films. When the polystyrene film is $300 \mathrm{~nm}$ the PWR angle is $34.80^{\circ}$. If the film thickness is increased to $350 \mathrm{~nm}$ the PWR angle will shift $1.75^{\circ}$ to $36.55^{\circ}$. While the thinnest film that can be measured using the sample geometry shown in Figure $1 \mathrm{~B}$ is fundamentally limited, there is no fundamental limit to the thickest film that can be studied with this geometry. One consideration is that multiple PWR peaks are possible, and the number of PWR peaks increases with increasing polymer thickness. Overall, this increases the complexity of the PWR Raman data set for films thicker than one micron.

The shape of the PWR angle versus polymer thickness plot indicates the minimum change in polymer thickness that can be measured using an instrument with an angle resolution of $0.05^{\circ}$ will vary across the thickness range. The smallest difference in polymer thickness that can be distinguished will be measured in the $450 \mathrm{~nm}$ range. Yet, accurate thicknesses are possible outside this range. For example, a polystyrene film thicknesses change from $500 \mathrm{~nm}$ to $510 \mathrm{~nm}$ will produce a $0.60^{\circ}$ shift. This shift is well within the angle resolution of most scanning angle instruments.

\section{Excitation wavelength effects on the OED distribution in polystyrene films}

The most favorable excitation wavelength for PWR Raman spectroscopy will sustain a guided mode with a high enough OED concentrated in the polymer film to obtain Raman spectra with adequate signal-to-noise ratios. Figure 4A shows the OED produced across the interface using five excitation wavelengths spanning the visible (488 $\mathrm{nm})$ to the near-IR $(1064 \mathrm{~nm})$ region of the electromagnetic spectrum. A $785 \mathrm{~nm}$ 
excitation wavelength produces the highest OED within the polymer film. A further consideration is the frequency to the fourth power dependence of the Raman scatter. For the same OED generated within the polymer film, visible light will produce more Raman scatter than near-IR wavelengths. For the study of a polystyrene waveguide, $532 \mathrm{~nm}$ excitation produces the largest integrate OED when the frequency to the fourth power dependence of Raman scatter is considered (Figure 4B).

On the other hand, visible excitation wavelengths are often associated with large spectral backgrounds. As determined by experimentation, near IR excitation wavelengths greater than approximately $650 \mathrm{~nm}$ but less than approximately $850 \mathrm{~nm}$ offer a good compromise of a high OED distribution without high fluorescence backgrounds. For near-IR excitation wavelengths greater than approximately $850 \mathrm{~nm}$ a majority of the total OED is located in the adjacent air medium.

\section{CONCLUSIONS}

This study shows how several experimental values affect the OED at the interface for the study of polystyrene waveguides. The film thickness and excitation wavelength will alter the OED distribution produced within the polystyrene film and, as expected, the PWR angle producing the maximum attenuation of the reflected light intensity. The PWR angle can be used to determine the film thickness, and $10 \mathrm{~nm}$ thickness differences can be easily measured with modest angle resolution. Results from this study provide valuable information on how OED distributions can be applied to interpret PWR Raman spectroscopy measurements. The work presented is not limited to polystyrene. All the 
presented results will be valid for polymers of the same refractive index as polystyrene, and similar calculations are valid for other dielectric polymers provided their refractive index fulfills the requirement for a waveguide. Current work involving the analysis of polymer multilayers using PWR Raman spectroscopy is being explored.

\section{ACKNOWLEDGEMENTS}

This research is supported by the U.S. Department of Energy, Office of Basic Energy Sciences, Division of Chemical Sciences, Geosciences, and Biosciences through the Ames Laboratory. The Ames Laboratory is operated for the U.S. Department of Energy by Iowa State University under Contract No. DEAC02-07CH11358. 


\section{REFRENCES}

1. M. W. Meyer, K. J. McKee and E. A. Smith, J. of Phys. Chem. C., 47, 24987 (2013).

2. K. J. McKee, M. W. Meyer and E. A. Smith, Anal. Chem., 84, 9049 (2012).

3. K. J. McKee and E. A. Smith, Rev. Sci. Instrum., 81, 043106 (2010).

4. A. Abbas, M. J. Linman and Q. Cheng, Sensor Actuat. B-Chem., 156, 169 (2011).

5. K. J. McKee, M. W. Meyer and E. A. Smith, Anal. Chem. 84, 4300 (2012). 


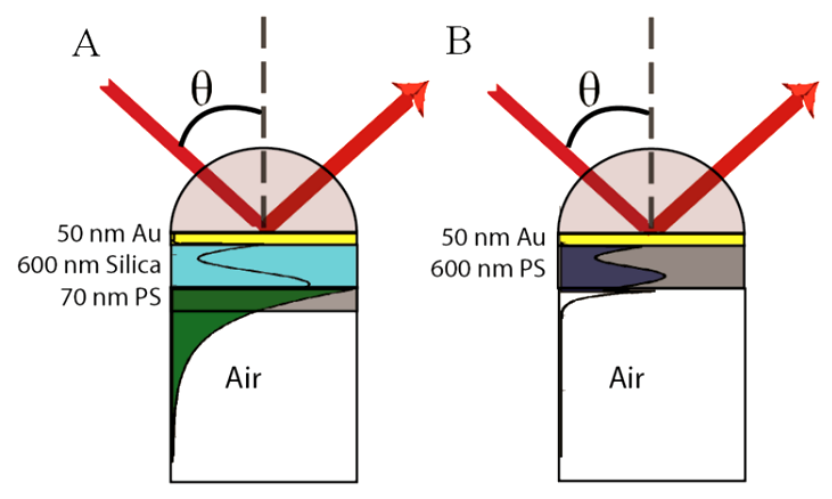

Figure 1. Two sample geometries for PWR Raman spectroscopy of thin polymer films:

(A) Polymer, for example polystyrene (PS), is deposited on a silica waveguide; (B)

Polymer is deposited directly on the gold film and the polymer acts as a waveguiding layer at thicknesses greater than $250 \mathrm{~nm}$. The electric field generated across the interface is shown by the black curve. The portion of the electric field that can generate Raman scatter from the polymer is shaded green (A) or purple (B). 

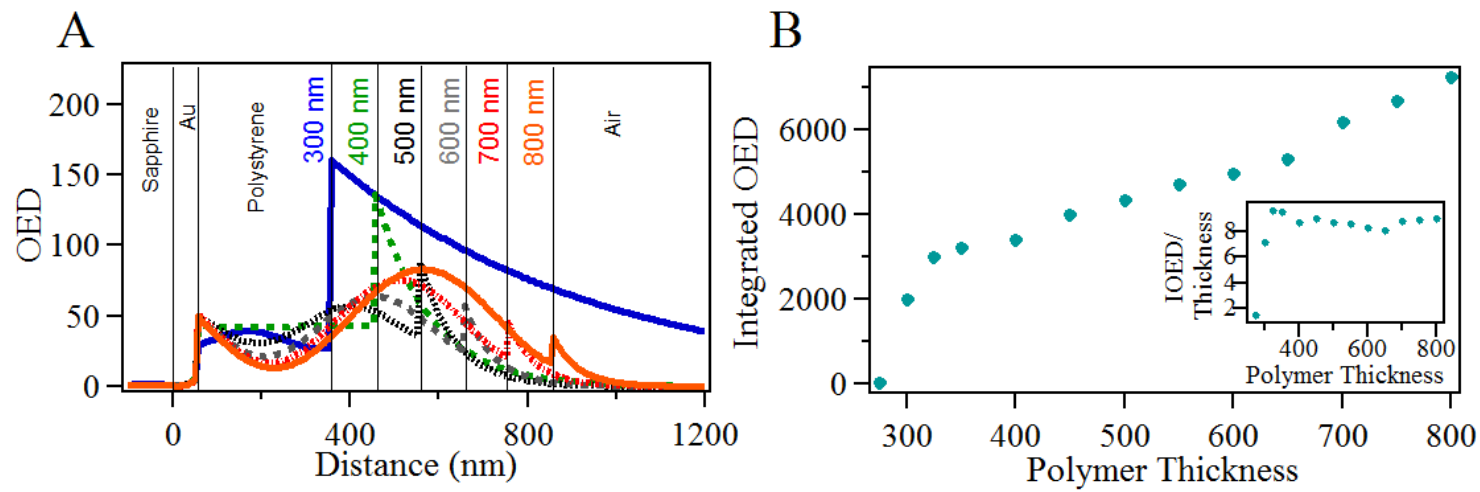

Figure 2. (A) Calculated optical energy density (OED) distribution throughout the interface using a sample geometry shown in Figure 1B with a polystyrene thickness of: $300 \mathrm{~nm}$ (blue), $400 \mathrm{~nm}$ (dashed green), $500 \mathrm{~nm}$ (dotted black), $600 \mathrm{~nm}$ (dashed grey), 700 $\mathrm{nm}$ (dotted red) and $800 \mathrm{~nm}$ (orange). The OED within the polymer film spans between the vertical line at the Au/polystyrene interface to the vertical line at the polystyrene/air interface, the latter varies with the polymer thickness. (B) The integrated OED across the entire polystyrene film of the indicated thickness. The inset shows the integrated OED (IOED) divided by the polymer thickness as a function of polymer thickness. This value remains relatively constant for all films thicker than approximately $325 \mathrm{~nm}$. The excitation wavelength for all calculations was $785 \mathrm{~nm}$. 


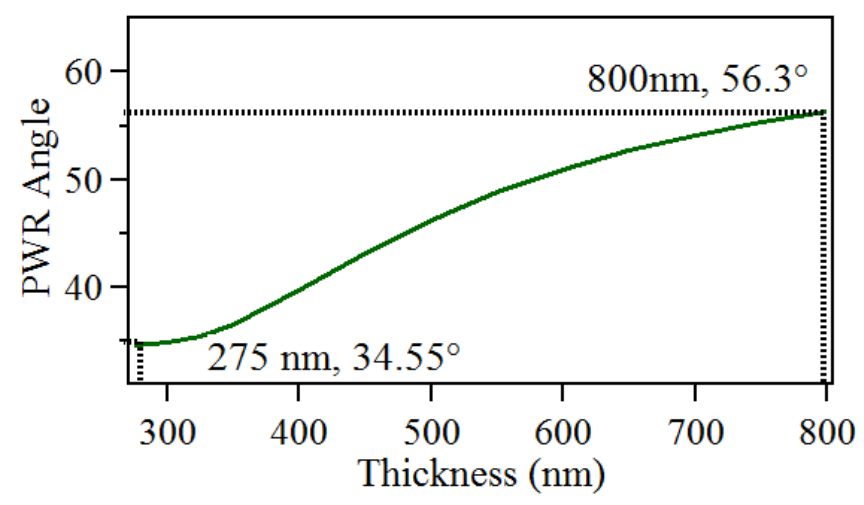

Figure 3. Calculated PWR angle as a function of film thickness for a sapphire/50 nm gold/polystyrene/air geometry. In a scanning angle PWR Raman spectroscopy experiment, the PWR angle is determined by the angle that produces the maximum Raman scatter as the incident angle is scanned. 

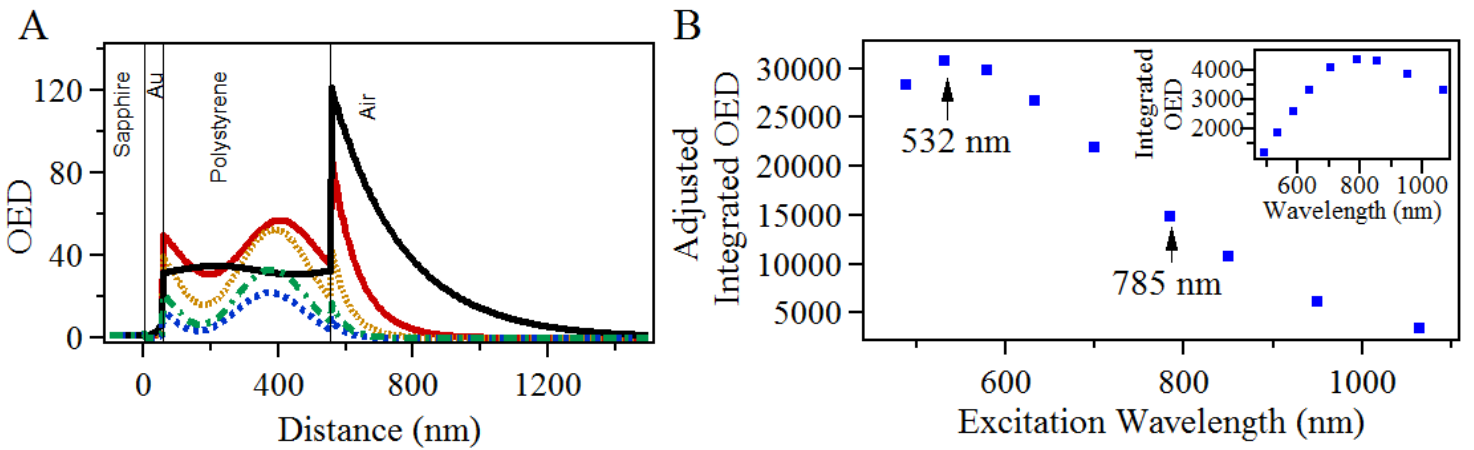

Figure 4. (A) Optical energy density (OED) distribution at the PWR angle for a $500 \mathrm{~nm}$ polystyrene film using a: $1064 \mathrm{~nm}\left(37.80^{\circ}\right.$ black $), 785 \mathrm{~nm}\left(46.20^{\circ} \mathrm{red}\right), 633 \mathrm{~nm}\left(51.65^{\circ}\right.$ dotted orange $), 532 \mathrm{~nm}\left(55.00^{\circ}\right.$ dotted-dashed green $)$ and $488 \mathrm{~nm}\left(56.35^{\circ}\right.$ dashed blue $)$ excitation wavelength. (B) The integrated OED (blue squares) calculated for a $500 \mathrm{~nm}$ polystyrene film adjusted to the frequency to the fourth power dependence of the Raman scattering intensity as a function of excitation wavelength. The optimum wavelength for PWR Raman measurements of polystyrene waveguides is $532 \mathrm{~nm}$. The inset shows the integrated OED prior to adjusting for the frequency to the fourth power dependence of the Raman signal. The maximum is at a $785 \mathrm{~nm}$ excitation wavelength. 


\title{
CHAPTER 5: OPTIMIZATION OF SILVER NANOPARTICLES FOR SURFACE ENHANCED RAMAN SPECTROSCOPY OF STRUCTURALLY DIVERSE ANALYTES USING VISIBLE AND NEAR- INFRARED EXCITATION
}

A paper published in Analyst, 2011, 17, 3542

Matthew W. Meyer and Emily A. Smith

Ames Laboratory, U. S. Department of Energy, Ames, Iowa 50011-3111, and Department of Chemistry, Iowa State University, Ames, Iowa 50011-3111

\begin{abstract}
Several experimental parameters affecting surface enhanced Raman (SER) signals using 488, 785 and $1064 \mathrm{~nm}$ excitation for eight diverse analytes are reported. Citrate reduced silver colloids having average diameters ranging from $40 \pm 10$ to $100 \pm 20 \mathrm{~nm}$ were synthesized. The nanoparticles were characterized by transmission electron microscopy, dynamic light scattering and absorbance spectrophotometry before and after inducing nanoparticle aggregation with $0.99 \%$ v/v $0.5 \mathrm{M}$ magnesium chloride. The nanoparticle aggregates and SERS signal were stable between 30 and 90 minutes after inducing aggregation. For the analytes 4-mercaptopyridine, 4-methylthiobenzoic acid and the
\end{abstract}


dipeptide phenylalanine-cysteine using all three excitation wavelengths, the highest surface area adjusted SER signal was obtained using $70 \pm 20 \mathrm{~nm}$ nanoparticles, which generated $290 \pm 40 \mathrm{~nm}$ aggregates with the addition of magnesium chloride. The decrease in the SER signal using non-optimum colloids was 12 to $42 \%$ using $488 \mathrm{~nm}$ excitation and larger decreases in signal, up to $92 \%$, were observed using near infrared excitation wavelengths. In contrast, pyridine, benzoic acid, and phenylalanine required $220 \pm 30 \mathrm{~nm}$ aggregates for the highest SER signal with 785 or $1064 \mathrm{~nm}$ excitation, but larger aggregates $(290 \pm 40 \mathrm{~nm})$ were required with $488 \mathrm{~nm}$ excitation. The optimum experimental conditions measured with the small molecule analytes held for a 10 amino acid peptide and hemoglobin. Reproducible SERS measurements with 2 to $9 \%$ RSD have been obtained by considering nanoparticle size, aggregation conditions, excitation wavelength and the nature of the analyte-silver interaction.

\section{INTRODUCTION}

Surface enhanced Raman spectroscopy (SERS) is a valuable tool for amplifying signals of weak Raman scatters. ${ }^{1-3}$ SERS with a single optimized metal nanoparticle can result in a $10^{2}$ to $10^{6}$ enhancement, and select aggregates of two or more nanoparticles generate enhancements up to $10^{10}$ to $10^{14}$. $^{4}$ SERS provides vibrational information about molecules in close proximity to roughened metallic surfaces. Several methods have been developed for the preparation of SERS-active surfaces. ${ }^{6-12}$ One of the most common and simply prepared SERS substrates are colloidal nanoparticles with optical properties that are dependent on the size and morphology of the particle. ${ }^{13-14}$ 
Aggregation of the nanoparticles with the addition of salts such as sodium chloride, magnesium sulfate or magnesium chloride produces higher enhancements than isolated nanoparticles due to the coupling of the localized surface plasmon dipoles. ${ }^{15-17}$ Silver nanoparticles have proven to be a common choice for SERS measurements in solution due to their broad plasmon resonance in the visible and near infrared regions, high stability, high surface enhancements and ease in fabrication. ${ }^{18}$ The citrate reduction of silver nitrate is a common synthesis that can be used to tune particle size and plasmon absorption at different laser wavelengths. Variations in the citrate concentration alter the nucleation-to-growth ratio to produce silver nanoparticles with average diameters between 30 and $200 \mathrm{~nm}^{19}$

A disadvantage of SERS can be a lack of reproducibility from measurement to measurement and laboratory to laboratory. ${ }^{20}$ This is due, in part, to the fact that SERS has many experimental parameters including the wavelength of excitation, the nature of the interaction between the analyte and metal, and the dependence of the enhancement on several nanoparticle properties. In regards to solution SERS measurements, nanoparticles with varied diameter, shape, surface charge, aggregation and stability produce varied surface enhancements. ${ }^{21}$ Optimization of the nanoparticles with regard to all these variables is of critical importance for the facile detection in solution of dilute analytes or analytes with small Raman cross sections.

To date, correlations between citrate reduced nanoparticle properties and achieved surface enhancements have been investigated using limited experimental parameters. ${ }^{21}$ Several authors have reported novel nanoparticle preparations and subsequently 
characterized their surface enhancement. ${ }^{22-25}$ McLellan et al. have reported that enhancement factors for 1,4-benzendithiol are highest for isolated (i.e., non-aggregated) $100 \mathrm{~nm}$ ethylene glycol/poly(vinyl pyrrolidine) reduced silver particles compared to 60 or $90 \mathrm{~nm}$ diameter particles using 514 and $785 \mathrm{~nm}$ excitation. They report particles of similar size with sharp edges produced more Raman scatter than corresponding spherical nanoparticles, and that the enhancement factor for isolated particles is correlated with localized surface plasmon absorption at the excitation laser wavelength. ${ }^{26}$ Zheng et al. reported $78 \pm 6 \mathrm{~nm}$ average diameter, isolated, borohydride reduced silver nanoparticles provided the greatest enhancement using $488 \mathrm{~nm}$ excitation and benzoic acid as the analyte when comparing particles with diameters ranging from 8 to $78 \mathrm{~nm}^{22}$ Emory et al. reported a screening method that enabled them to deposit isolated nanoparticles onto substrates and screen the surface enhancement of individual particles. They found that most SERS active particles using $514 \mathrm{~nm}$ excitation are in the range of 80 to $100 \mathrm{~nm} .^{27}$ Bell et al. reported that aggregated gold particles exhibited the greatest thiophenol surface enhancement with $74 \mathrm{~nm}$ particles in the studied 21 to $146 \mathrm{~nm}$ range using 785 nm excitation, and that the enhancements do not correlate with absorbance at the excitation wavelength. ${ }^{28}$ The size of the nanoparticle aggregates resulting from addition of magnesium sulfate was not characterized, and there may not have been a direct correlation between nanoparticle size and aggregate size. The correlation between optimal aggregate size and large surface enhancements for solution SER measurements has not been determined for various analytes and excitation wavelengths.

Given the commonplace use of aggregated silver nanoparticles for SERS measurements, a systematic study is needed to correlate surface enhancements from 
aggregated silver nanoparticles with the mode of interaction between the analyte and metal surface, analyte structure, excitation wavelength, size of the nanoparticle aggregates, as well as, the stability of the Raman measurements over time. Herein, the optimum properties of citrate reduced silver nanoparticles for SERS detection using 488, 785 and $1064 \mathrm{~nm}$ excitation with analytes that interact with the metal surface through different mechanisms are presented. To our knowledge, this is the first comparative investigation of the reproducibility and optimization of citrate reduced silver colloids for the detection of analytes with diverse chemical functionality and varying excitation wavelengths. UV visible spectrophotometry, transmission electron microscopy (TEM) and dynamic light scattering (DLS) have been used to measure the size, polydispersity and surface charge of citrate reduced nanoparticles before and after aggregation by addition of salt. Surface enhancements have been measured for pyridine, 4mercaptopyridine, benzoic acid, 4-methylthiobenzoic acid, phenylalanine, cysteinephenylalanine, a ten amino acid peptide and a protein. The results show detrimental consequences of not carefully considering all experimental parameters prior to SERS analysis, and highlight several considerations that can improve SERS reproducibility.

\section{EXPERIMENTAL}

\section{Materials}

The phenylalanine-cysteine peptide was purchased from Bachem Americas, Inc. All other chemicals were analytical grade from Sigma Aldrich. Aqueous solutions were

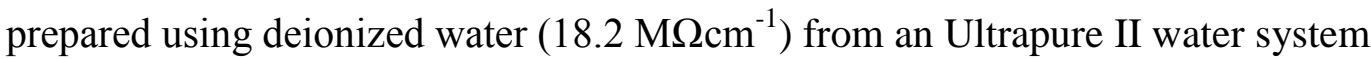
(Thermo Scientific, Waltham, MA). 


\section{Preparation of silver colloids}

Silver colloids were prepared by the classical citrate reduction of silver nitrate first reported by Lee and Meisel with some modifications. ${ }^{29}$ A total volume of $40 \mathrm{~mL}$ $\mathrm{AgNO}_{3}(100 \mu \mathrm{M})$ was brought to a boil. One percent w/v sodium citrate $(0.2,0.5,0.8$, 1.0 , or $1.5 \mathrm{~mL}$ ) was then added and the solution refluxed for 30 minutes. The temperature, stirring rates and rate of sodium citrate addition were held constant for each synthesis. The $\mathrm{pH}$ of all colloid solutions was adjusted to 6.5 using sodium hydroxide. The nanoparticles were stored in the dark at $4^{\circ} \mathrm{C}$.

\section{Nanoparticle characterization}

The absorbance spectra of the nanoparticles were recorded on an Agilent 8453 spectrophotometer. Nanoparticle concentration was calculated as previously described (density of face centered cubic silver is $\left.10.5 \mathrm{~g} / \mathrm{cm}^{3}\right) .{ }^{30}$ For TEM measurements, nanoparticle solutions were diluted 10-fold and drop casted on a Formvar/carbon coated transmission electron microscope grid (Ted Pella, Redding, CA) and allowed to dry

overnight. Imaging was performed on a Philips Tecnai $\mathrm{G}^{2}$ high resolution TEM operated at an accelerating voltage of $200 \mathrm{kV}$. The average diameter of a minimum of 100 particles was quantified with Image J software. Particle surface area was calculated using the average diameter measured by TEM. Hydrodynamic diameters of the nanoparticles were measured by DLS using a Malvern Zetasizer Nano ZS (Worcestershire, UK). The dynamic range of the DLS instrument according to the manufactuer's specifications is 3 $\mathrm{nm}$ to $6 \mu \mathrm{m}$. Solutions of nanoparticles, analyte and salt at the same concentration used for Raman measurements were diluted 1:20 in water and analyzed by DLS. DLS 
measurements were performed in triplicate at $25^{\circ} \mathrm{C}$ with each measurement the average of 15 runs.

\section{SERS measurements}

Nanoparticles $(450 \mu \mathrm{L})$ were added to analyte $(50 \mu \mathrm{L})$ and equilibrated at ambient conditions for 30 minutes. All analyte solutions were prepared in water except benzoic acid and 4-methylthiobenzoic acid, which were prepared in a mixture of $25 \mu \mathrm{L}$ ethanol and $25 \mu \mathrm{L}$ water. The final analyte concentration was $2.0 \times 10^{-3} \mathrm{M}$ except for angiotensin $\mathrm{I}\left(2.0 \times 10^{-4} \mathrm{M}\right)$ and hemoglobin $\left(1.0 \times 10^{-5} \mathrm{M}\right)$. The nanoparticles were aggregated by the addition of $5 \mu \mathrm{L} 0.5 \mathrm{M}$ magnesium chloride to give a final salt concentration of $0.99 \% \mathrm{v} / \mathrm{v}$, except where noted in the text. The solution turned from a green to a bluegray color, which indicates particle aggregation. The solution was gently shaken for one minute and then incubated for 30 minutes at ambient conditions to ensure reproducible SERS measurements. The Raman spectra were collected 30 minutes after mixing the nanoparticle solution with the salt, unless otherwise noted in the text. Raman measurements were recorded on three home-built Raman spectrometers with excitation wavelengths of 488, 785 and $1064 \mathrm{~nm}^{31}$ Briefly, the $1064 \mathrm{~nm}$ spectrometer consisted of a f/4.1 spectrograph (Horiba Scientific) and liquid nitrogen cooled 512 x 1 pixel InGaAs detector with a Nd:YVO 4 1-Watt maximum output laser. The $785 \mathrm{~nm}$ and $488 \mathrm{~nm}$ spectrometer consisted of a f/1.8i spectrograph (Kaiser Optical Systems Inc.), PIXIS NIR enhanced CCD camera (Princeton Instruments) with a $140 \mathrm{~mW} 785 \mathrm{~nm}$ diode laser or a $50 \mathrm{~mW}$ argon ion laser. The laser power at the sample was adjusted to appoximtaly account for the Raman scatter's frequency to the fourth power dependence: $247 \mathrm{~mW}$ 
$(1064 \mathrm{~nm}), 70 \mathrm{~mW}(785 \mathrm{~nm})$ and $15 \mathrm{~mW}(488 \mathrm{~nm})$. All lasers were $\geq 95 \%$ p-polarized. The accumulation time was 10 seconds and 3 accumulations were averaged for each spectrum. Three replicate measurements were performed for each measurement.

\section{Data analysis}

Raman data were acquired using the software program Winview (488 \& $785 \mathrm{~nm})$ or SynerJY (1064 nm). Baseline correction or background subtraction were not required with these data. The raw data were exported into Grams AI and adjusted for acquisition time by converting intensity to counts per second. Where noted in the text, the data are also adjusted for the total nanoparticle surface area in the analysis volume (counts

second $^{-1} \mathrm{~nm}^{-2}$ ). Surface enhancement (EF) was calculated using equation 1 :

$$
\mathrm{EF}=\frac{\mathrm{I}_{\text {SERS }} \mathrm{C}_{\text {SOL }}}{\mathrm{I}_{\mathrm{SOL}} \mathrm{C}_{\mathrm{SERS}}}
$$

where I is the intensity of a Raman peak for analyte in solution ( $\left.\mathrm{I}_{\mathrm{SOL}}\right)$ or in the presence of silver nanoparticles $\left(\mathrm{I}_{\mathrm{SERS}}\right)$ and $\mathrm{C}$ is the analyte concentration in solution $\left(\mathrm{C}_{\mathrm{SOL}}\right)$ or adsorbed to the colloid $\left(\mathrm{C}_{\mathrm{SERS}}\right)$ within the probed beam area.

\section{RESULTS AND DISCUSSION}

\section{Silver colloid characterization}

In order to determine the optimum nanoparticle properties for SERS measurements, several batches of silver particles were synthesized using the Lee and Meisel method with different citrate starting concentrations. ${ }^{29}$ The nanoparticles were 
characterized using absorption spectrophotometry, TEM and DLS (Table 1, Supporting

Information Figure S1, S2), and the results are consistent with previous reports. ${ }^{21}$ The TEM measurements confirmed that lower citrate concentrations generated larger diameter particles and a red shift in the particles' absorbance maximum. The TEM images also reveal that 2 to $6 \%$ of the particles were rod shaped, rather than spherical. The standard deviation in particle diameter was 10 to $20 \mathrm{~nm}$. The colloid's surface charge is consistent with stable particles and ranged from $-31 \mathrm{mV}$ to $-42 \mathrm{mV}$.

\section{Nanoparticle aggregation}

Larger surface enhancements can be achieved when silver nanoparticles are aggregated prior to collecting the Raman signal. The optimum aggregating agent, concentration of aggregating agent and incubation time were studied. Multiple salts were tested as aggregating agents including chloride and sulfate salts using pyridine as the analyte (Supporting Information Figure S3). Chloride salts produced higher signal-tonoise ratios than sulfate salts and showed no interference peaks from citrate with all particles included in this study. A $0.5 \mathrm{M}$ stock solution of magnesium chloride was added to a mixture of pyridine and $70 \mathrm{~nm}$ colloids to give a final salt concentration ranging from 0.1 to 9 percent volume/volume. The Raman spectra were collected after 30 minutes. The largest Raman signal was produced using 4.8 percent magnesium chloride (Figure 1a). Concentrations greater than five percent caused the particles to rapidly precipitate out of solution, and produced less Raman scatter.

Aside from high levels of Raman scatter, another consideration is the stability of the Raman signal over time. Rapid flocculation of the aggregates must be avoided to 
produce stable surface enhancements. Larger Raman signals were obtained using 4.8 percent magnesium chloride (Figure 1b) compared to lower salt concentrations, but the window of time over which the highest SERS signals could be obtained within 3\% RSD was less than 9 minutes (depending on the aggregate size). If 0.99 percent magnesium chloride was used as an aggregating agent (Figure 1c), stable SERS measurements within 3\% RSD were obtained for all particles sizes between 30 and 90 minutes after salt addition. All subsequent studies used 0.99 percent magnesium chloride as an aggregating agent.

The average aggregate hydrodynamic diameters were measured by DLS 30 minutes after adding one percent magnesium chloride and are reported in Table 1. The distributions are shown in Supporting Information Figure S4. The analysis time and experimental conditions for the DLS measurements were consistent with those used for the Raman measurements. There is no direct correlation between nanoparticle size and aggregate size; however, there was a general trend for the largest particles to form larger aggregates. The polydispersity of the aggregates is 2 to 3 times larger compared to the unaggregated nanoparticles.

The aggregate size as measured by DLS was stable between 30 to 90 minutes after adding one percent magnesium chloride (Supporting Information Figure S5), which was consistent with the stable Raman signal measured for the same time frame. Over 6 to 150 hours, depending on the nanoparticle stability after salt addition, the nanoparticles precipitated to the bottom of the sample holder and the sample no longer produced SER signal. The aggregates' zeta potential decreased after the addition of salt (Table 1). For 
the smaller aggregates $(220 \pm 30$ to $340 \pm 20 \mathrm{~nm})$ the surface charge was in the range -13 $\pm 5 \mathrm{mV}$ to $-16 \pm 4 \mathrm{mV}$. Larger aggregates had surface charges in the range $-7.5 \pm 2 \mathrm{mV}$ to $+5 \pm 3 \mathrm{mV}$. The larger aggregates precipitate out of solution over a shorter time frame (i.e., hours) than the smaller aggregates, but they still produce stable Raman signals in the 30 minute time frame used for these studies.

\section{Small molecule SERS measurements}

SER spectra for each analyte and aggregate size are shown in Figure 2a to f using $1064 \mathrm{~nm}$ excitation. Three probe molecules containing thiol groups (4-mercaptopyridine, 4-methylthiobenzoic acid, phenylalanine-cysteine) and three probe molecules without thiol groups but structurally similar to the thiol containing analytes (pyridine, benzoic acid, phenylalanine), were tested. The non-thiol containing analytes produce more scatter than the thiol-containing analytes. This is likely due to a lower concentration of the thiolcontaining analytes at the silver surface, the orientation of the analyte with respect to the metal surface and possible differences in the chemical enhancement mechanism. Adsorption isotherms (Supplemental Information Figure S6) reveal that $2.0 \times 10^{-3} \mathrm{M}$ represents $13 \%, 9 \%, 46 \%$ and $44 \%$ surface coverage for pyridine, mercaptopyridine, benzoic acid, and 4-methylthiolbenzoic acid, respectively. Benzoic acid interacts with silver through the carboxylate group and is oriented perpendicular to the surface; whereas 4-methylthiobenzoic acid interacts with the surface primarily through the carboxylate group and sulfur atom, and is oriented parallel to the surface. ${ }^{32-33}$ Due to the analyte adsorption orientation, the packing density of benzoic acid is larger than 4methylthiobenzoic acid. This argument holds for pyridine, which interacts perpendicular 
to the surface through its nitrogen atom, and 4-mercaptopyridine, which interacts primarily through the sulfur and nitrogen atoms and is oriented parallel to the silver surface. ${ }^{34-35}$ Both phenylalanine and phenylalanine-cysteine are oriented parallel to the surface. ${ }^{36-37}$ The differing nature of the interaction between the anlayte and the silver surface also causes varying amounts of chemical enhancement. ${ }^{38}$ This typically accounts for $\sim 10^{2}$ of the total surface enhancement. The remainder of the surface enhancement is from the electromagnetic enhancement.

The generated Raman scatter for the $\sim 1000 \mathrm{~cm}^{-1}$ ring breathing mode (or $1074 \mathrm{~cm}^{-}$ ${ }^{1}$ mode for 4-methylthiobenzoic acid) showed a clear dependence on the aggregate size for all analytes (Figure 2). Less Raman scatter was collected for the larger aggregates, with no Raman signal collected for phenylalanine-cysteine using the largest aggregates.

The total colloid volume was held constant for each measurement; therefore, the concentration of particles and particle surface area varied for each nanoparticle sample (Table 2). The surface area correlates with the amount of analyte that can interact with the particle, which will affect the SER signal. The scattering intensity of each analyte was adjusted for the nanoparticle surface area (Figure 3). For pyridine, benzoic acid and phenylalanine (Figure 3a) using $1064 \mathrm{~nm}$ excitation, the smallest aggregates in the $220 \pm$ 30 to $290 \pm 40 \mathrm{~nm}$ size regime generated similar amounts of surface area adjusted Raman scatter. For the related thiol-containing analytes (Figure 3b) using $1064 \mathrm{~nm}$ excitation, $290 \pm 40 \mathrm{~nm}$ aggregates were optimal when the signal was adjusted for particle surface area. The average signal decrease using $220 \pm 20$ or $910 \pm 40 \mathrm{~nm}$ aggregates (50 \pm 10 or $80 \pm 20 \mathrm{~nm}$ colloids) with pyridine, benzoic acid and phenylalanine was $55 \%$ and $47 \%$, 
respectively. This was similar to the decrease in signal for pyridine, benzoic acid and phenylalanine using non-optimal aggregates greater than $340 \pm 20 \mathrm{~nm}$ in size.

There was no correlation between the plasmon absorption at the excitation wavelength (Supplemental Information Figure S2) and the maximum amount of measured surface enhancement. This is consistent with the results from Bell et al. using aggregated particles. $^{28}$ The optimum aggregate size for each analyte had no correlation with the percentage of rods (aspect ratio greater than 1.5) in the colloid (Table 1). Sharp edges have been shown to produce higher enhancements than spherical particles. ${ }^{39}$ The aggregates that produced the largest surface enhancements have the fewest number of nanoparticles per aggregate, but not necessarily the largest number of aggreagtes probed by the excitation beam (Table 2). This confirms aggregate morphology is an important parameter for high surface enhancements. The difference in optimum aggregate size with thiol- and nonthiol-containing analytes may be due to differences in the aggregate morphology or geometry that were not measured by DLS. For example, a majority of the SER signal comes from 'hot spots', which represent a fraction of the total silver surface area. $^{15}$ The properties of these hot spots may be altered by the thiol-containing analytes in a way that alters the optimum aggregate size for maximum surface enhancement.

Overall, these results reveal that smaller aggregates of citrate reduced silver nanoparticles produce the highest surface enhancements, in general, but the nature of the interaction between the analyte-silver surface should be considered to achieve the largest enhancement.

Optimum aggregate size: Excitation wavelength 
The surface area adjusted Raman signal for pyridine and 4-mercaptopyridine obtained using 488, 785 or $1064 \mathrm{~nm}$ excitation and each aggregate size included in this study is shown in Figure $4 \mathrm{a}$ and $\mathrm{b}$. The signal has been normalized to the highest signal obtained for each wavelength and analyte, which is useful to identify trends in the optimum aggregate size for each excitation wavelength. The optimum aggregate size for 4-mercaptopyridine was consistent at all excitation wavelengths, although the decrease in signal for the non-optimal aggregates was not. The signal decrease using aggregates larger than $340 \mathrm{~nm}$ was significantly higher for $785 \mathrm{~nm}$ excitation compared to 488 or $1064 \mathrm{~nm}$. For all wavelengths, the dependence of the SER signal on aggregate size was complex and did not show a clear trend. For pyridine the $290 \pm 40 \mathrm{~nm}$ aggregates generated the largest Raman signal using $488 \mathrm{~nm}$ excitation; whereas the $220 \pm 30 \mathrm{~nm}$ aggregates were optimal for near-IR excitation. The aggregates larger than $910 \pm 40 \mathrm{~nm}$ produced much less SER signal at all wavelengths for pyridine. Overall, the results reveal that similar aggregate sizes in the 220 to $290 \mathrm{~nm}$ size regime can be used to generate large SER signals across excitation wavelengths.

The surface enhancement is a ratio of the surface enhanced to normal Raman signals (Equation 1). Enhancements were calculated for the most intense Raman peak for each analyte with 488, 785 and $1064 \mathrm{~nm}$ excitation wavelengths and $220 \pm 30$ or $290 \pm$ $40 \mathrm{~nm}$ aggregates (Table 2). Also shown in Table 2 are the measured surface enhancements for pyridine using $50 \pm 5 \mathrm{~nm}$ gold colloids with all other experimental conditions the same as previously described. For the gold aggregates, the enhancements were 6,50 and $0.1 \%$ of the values obtained for silver aggregates at 1064,785 and 488 $\mathrm{nm}$, respectively. Higher surface enhancements for silver compared to gold are predicted 
based on the metals' dielectric constant. ${ }^{40}$ However, the optimum aggregate conditions for the gold particles may not be the same as those for the silver particles.

Compared to $785 \mathrm{~nm}$ excitation, the average surface enhancement across all analytes using silver colloids and this protocol was 67 and 22\% smaller at 1064 and 488 $\mathrm{nm}$, respectively. The throughputs of the spectrometers used in this study, the excitation power and laser profile are different for each excitation wavelength; however, this does not affect the surface enhancement, which is a ratio of the SERS to normal Raman signal. The higher surface enhancements with near IR excitation are explained by the large aggregate absorbance at these wavelengths compared to $488 \mathrm{~nm}$. For pyridine, benzoic acid and phenylalanine the surface enhancement was 1.2 to 1.4 times higher using 785 $\mathrm{nm}$ compared to $1064 \mathrm{~nm}$ excitation. Whereas for 4-mercaptopyridine, 4mercaptothiolbenzoic acid and phenylalanine-cysteine the surface enhancement was 1.7 to 2.0 times higher using $785 \mathrm{~nm}$ excitation.

\section{Peptide and protein SERS measurements}

The question remains whether the optimum experimental conditions measured for the small molecule analytes can be a starting point for optimizing experimental parameters using more complex analytes. To test this, the SER spectra were collected for a 10 amino acid peptide and a protein (Figure 5). The peptide does not contain cysteine. The protein contains two cysteine amino acids; however, the protein structure reveals that they are buried in the protein interior. The ring-breathing mode had contributions from the amino acids phenylalanine, histidine, tryptophan and tyrosine. Overall less Raman scatter was collected compared to the small molecule analytes, likely due to the increased 
separation distance between the silver surface and the functional groups producing the Raman scatter and possibly from lower adsorbate concentrations. Comparing the ring breathing mode intensities $\left(\sim 1000 \mathrm{~cm}^{-1}\right)$ using the different aggregate sizes reveals that the peptide and protein followed the same trend as was measured for pyridine, benzoic acid and phenylalanine. The $220 \pm 30 \mathrm{~nm}$ aggregates produced the largest SER signal. The $290 \pm 40 \mathrm{~nm}$ aggregates produce only $67 \%$ (peptide) or $82 \%$ (protein) of the Raman scatter compared to the optimum aggregate size. For aggregates larger than $340 \pm 20 \mathrm{~nm}$, no detectable Raman peaks were measured. If the aggregates size is not carefully controlled and measured, no or non-reproducible SER signal may result. Surface enhancements were not calculated because normal Raman spectra could not be obtained for these analytes under the conditions used in this study. This highlights the possibility of using the results from the small molecule analytes as a starting point for optimizing conditions for analyses of more complex analytes. Of course, differences in the nature of the analyte-metal interaction, the analyte structure and solvent could affect the required conditions for optimum SER signal.

\section{CONCULSIONS}

Overall, the largest surface enhancements using this protocol and 1064, 785 and $488 \mathrm{~nm}$ excitation were measured with silver nanoparticle aggregates in the 220 to 290 nm size regime. Larger aggregates $(>340 \mathrm{~nm}$ ) produced no detectable Raman scatter for some analytes. The nature of the adsorbate-metal interaction should be considered when determining the optimum experimental parameters for high surface enhancements, or else a significantly lower signal may result. Using this protocol, stable SERS measurements 
with relative standard deviations between 2 and $9 \%$ were made from day to day, and using different batches of colloids with the same synthesis conditions. Correlations between surface enhancement and peptide structure/amino acid content are currently under investigation.

\section{ACKNOWLEDGEMENTS}

This work was supported by the Director of Science, Office of Basic Energy Sciences, Division of Chemical Sciences. The Ames Laboratory is operated for the U.S. Department of Energy by Iowa State University under Contract No. DE-AC0207CH11358. The authors thank Brian Trewyn (Iowa State University) for the use of the DLS instrument. 


\section{REFRENCES}

1. M. Knauer, N. P. Ivleva, X. Liu, R. Niessner and C. Haisch, Anal. Chem. (Washington, DC, U. S.), 2010, 82, 2766-2772.

2. K. Kneipp, H. Kneipp and J. Kneipp, Acc. Chem. Res., 2006, 39, 443-450.

3. G. D. Chumanov, R. G. Efremov and I. R. Nabiev, J. Raman Spectrosc., 1990, 21, 43-48.

4. K. Kneipp, Y. Wang, H. Kneipp, L. T. Perelman, I. Itzkan, R. R. Dasari and M. S. Feld, Phys. Rev. Lett., 1997, 78, 1667-1670.

5. J. A. Dieringer, R. B. Lettan, II, K. A. Scheidt and R. P. Van Duyne, J. Am. Chem. Soc., 2007, 129, 16249-16256.

6. D. A. Stuart, J. M. Yuen, N. Shah, O. Lyandres, C. R. Yonzon, M. R. Glucksberg, J. T. Walsh and R. P. Van Duyne, Anal. Chem., 2006, 78, 7211-7215.

7. B. Nikoobakht and M. A. El-Sayed, J. Phys. Chem. A, 2003, 107, 33723378.

8. G. Schmid, Chem. Rev., 1992, 92, 1709-1727.

9. S. Sanchez-Cortes, J. V. Garcia-Ramos, G. Morcillo and A. Tinti, J. Colloid Interface Sci., 1995, 175, 358-368. 
10. C. J. Addison and A. G. Brolo, Langmuir, 2006, 22, 8696-8702.

11. M. G. Albrecht and J. A. Creighton, J. Am. Chem. Soc., 1977, 99, 52155217.

12. R. S. Sheng, L. Zhu and M. D. Morris, Anal. Chem., 1986, 58, 1116-1119.

13. L. M. Liz-Marzan, Langmuir, 2006, 22, 32-41.

14. M. Futamata, Y. Maruyama and M. Ishikawa, Vib. Spectrosc., 2002, 30, $17-23$.

15. M. Moskovits, J. Raman Spectrosc., 2005, 36, 485-496.

16. T.-W. Koo, S. Chan, L. Sun, X. Su, J. Zhang and A. A. Berlin, Appl. Spectrosc., 2004, 58, 1401-1407.

17. A. M. Michaels, M. Nirmal and L. E. Brus, J. Am. Chem. Soc., 1999, 121, 9932-9939.

18. J. C. Cook, C. M. P. Cuypers, B. J. Kip, R. J. Meier and E. Koglin, J. Raman Spectrosc., 1993, 24, 609-619.

19. A. Henglein and M. Giersig, J. Phys. Chem. B, 1999, 103, 9533-9539.

20. M. J. Natan, Faraday Discuss., 2006, 132, 321-328.

21. M. V. Canamares, J. V. Garcia-Ramos, J. D. Gomez-Varga, C. Domingo and S. Sanchez-Cortes, Langmuir, 2005, 21, 8546-8553. 
22. K. H. Zheng, Y. C. Chou, Y. J. Wu and Y. T. Lee, J. Raman Spectrosc., 2010, 41, 632-635.

23. C. H. Munro, W. E. Smith, M. Garner, J. Clarkson and P. C. White, Langmuir, 1995, 11, 3712-3720.

24. C. S. Seney, B. M. Gutzman and R. H. Goddard, J. Phys. Chem. C, 2009, $113,74-80$.

25. N. Leopold and B. Lendl, J. Phys. Chem. B, 2003, 107, 5723-5727.

26. J. M. McLellan, A. Siekkinen, J. Chen and Y. Xia, Chem. Phys. Lett., 2006, 427, 122-126.

27. S. R. Emory and S. Nie, J. Phys. Chem. B, 1998, 102, 493-497.

28. S. E. J. Bell and M. R. McCourt, Phys. Chem. Chem. Phys., 2009, 11, $7455-7462$.

29. P. C. Lee and D. Meisel, J. Phys. Chem., 1982, 86, 3391-3395.

30. X. Liu, M. Atwater, J. Wang and Q. Huo, Colloids Surf., B, 2007, 58, 3-7.

31. C.-J. Shih and E. A. Smith, Anal. Chim. Acta, 2009, 653, 200-206.

32. Y. J. Kwon, S. B. Lee, K. Kim and M. S. Kim, J. Mol. Struct, 1994, 318, 25-35.

33. Y. J. Kwon, D. H. Son, S. J. Ahn, M. S. Kim and K. Kim, J. Phys. Chem., $1994,98,8481-8487$. 
34. A. Kaczor, K. Malek and M. Baranska, J. Phys. Chem. C, 2010, 114, 3909-3917.

35. L. Zhang, Y. Bai, Z. Shang, Y. Zhang and Y. Mo, J. Raman Spectrosc., 2007, 38, 1106-1111.

36. G. D. Fleming, J. J. Finnerty, M. Campos-Vallette, F. Celis, A. E. Aliaga, C. Fredes and R. Koch, J. Raman Spectrosc., 2009, 40, 632-638.

37. A. Singha, S. Dasgupta and A. Roy, Biophys. Chem., 2006, 120, 215-224.

38. K. Faulds, A. Hernandez-Santana and W. E. Smith, Spectrosc. Prop. Inorg. Organomet. Compd., 41, 1-21.

39. M. Rycenga, P. H. C. Camargo, W. Li, C. H. Moran and Y. Xia, J. Phys. Chem. Lett., 2010, 1, 696-703.

40. M. Moskovits, in Surface-Enhanced Raman Scattering Physics and Applications, ed. K. Kneipp, M. Moskovits, H. Kneipp, Springer, New York, Ny, 2006, pp. 1-14. 
Table 1. Measured parameters for citrate reduced silver nanoparticles before and after aggregation with $0.99 \% \mathrm{v} / \mathrm{v} 0.5 \mathrm{M}$ magnesium chloride.

\begin{tabular}{|c|c|c|c|c|c|c|c|}
\hline $\begin{array}{l}\text { Nanoparticle } \\
\text { Diameter } \\
(\mathrm{nm})\end{array}$ & $\begin{array}{c}1 \% \mathrm{w} / \mathrm{v} \\
\text { Citrate } \\
(\mathrm{mL})\end{array}$ & $\begin{array}{c}\text { Absorbance } \\
\text { Max (nm) }\end{array}$ & $\begin{array}{c}\text { Absorbance } \\
\text { Full Width } \\
\text { Half-Max (nm) }\end{array}$ & $\begin{array}{c}\text { Zeta } \\
\text { Potential } \\
(\mathrm{mV})^{\mathrm{a}}\end{array}$ & $\begin{array}{c}\% \\
\text { Rods }^{b}\end{array}$ & $\begin{array}{l}\text { Aggregate Hydro- } \\
\text { dynamic Diameter } \\
\text { with } \mathrm{MgCl}_{2}(\mathrm{~nm})^{\mathrm{a}}\end{array}$ & $\begin{array}{l}\text { Zeta Potential } \\
\text { with } \mathrm{MgCl}_{2} \\
(\mathrm{mV})^{\mathrm{a}}\end{array}$ \\
\hline $40 \pm 10$ & 1.5 & 405 & 70 & $-36 \pm 9$ & 5.1 & $340 \pm 20$ & $-13 \pm 5$ \\
\hline $70 \pm 20$ & 0.8 & 440 & 140 & $-34 \pm 9$ & 2.4 & $290 \pm 40$ & $-16 \pm 4$ \\
\hline $80 \pm 20$ & 0.5 & 460 & 200 & $-42 \pm 7$ & 6.4 & $910 \pm 40$ & $-8 \pm 2$ \\
\hline
\end{tabular}

a \pm 1 standard deviation from 3 replicate experiments.

b Aspect ratio greater than 1.5. 
Table 2. Calculated parameters for citrate reduced silver nanoparticles before and after aggregation with $0.99 \% \mathrm{v} / \mathrm{v} 0.5 \mathrm{M}$ magnesium chloride.

\begin{tabular}{|c|c|c|c|c|}
\hline $\begin{array}{c}\text { Nanoparticle } \\
\text { Diameter }(\mathrm{nm})\end{array}$ & $\begin{array}{c}\text { Surface Area per } \\
\text { Nanoparticle } \\
\left(\mathrm{nm}^{2}\right)\left(\mathrm{x} \mathrm{10}^{4}\right)\end{array}$ & $\begin{array}{c}\text { Nanoparticle } \\
\text { Concentration } \\
(\mathrm{mol} / \mathrm{L}) \\
(\mathrm{x} \mathrm{10})\end{array}$ & $\begin{array}{c}\text { Average Number } \\
\text { of Nanoparticles } \\
\text { per Aggregate }\end{array}$ & $\begin{array}{c}\text { Average } \\
\text { Number of Aggregates } \\
\text { in Excitation Volume }\end{array}$ \\
\hline $40 \pm 10$ & 0.50 & 7.66 & 8.5 & 76 \\
\hline $50 \pm 10$ & 0.78 & 3.92 & 4.4 & 70 \\
\hline $70 \pm 20$ & 1.5 & 1.42 & 4.1 & 6 \\
\hline $80 \pm 20$ & 2.0 & 0.96 & 11.4 & 4 \\
\hline $100 \pm 20$ & 3.1 & 0.49 & 9.6 & 26 \\
\hline
\end{tabular}

${ }^{a}$ Calculated for $1064 \mathrm{~nm}$ excitation beam profile, assuming a homogeneous distribution of aggregates in sample holder. Divide by 33 or 411 for 785 or $488 \mathrm{~nm}$ excitation, respectively. 
Table 3. Calculated surface enhancements (SE) for small molecule analytes using 1064,785 or $488 \mathrm{~nm}$ excitation.

\begin{tabular}{|l|c|c|c|c|c|}
\hline Compound & $\begin{array}{c}\text { Analyzed } \\
\left.\text { Peak (cm }{ }^{-1}\right)\end{array}$ & $\begin{array}{c}\text { Aggregate Size } \\
\text { for SE } \\
\text { Calculation }(\mathrm{nm})\end{array}$ & $\begin{array}{c}1064 \mathrm{~nm} \mathrm{SE} \\
\left(\mathrm{x} 10^{5}\right)^{\mathrm{c}}\end{array}$ & $\begin{array}{c}785 \mathrm{~nm} \\
\mathrm{SE} \\
\left(\mathrm{x} 10^{5}\right)^{\mathrm{d}}\end{array}$ & $\begin{array}{c}488 \mathrm{~nm} \text { SE } \\
\left(\mathrm{x} 10^{5}\right)^{\mathrm{e}}\end{array}$ \\
\hline Pyridine $(\mathrm{Ag})^{\mathrm{a}}$ & 1006 & $220 \pm 30$ & $10.7 \pm 0.6$ & $\begin{array}{c}12.9 \pm \\
0.7\end{array}$ & $4.9 \pm 0.2$ \\
\hline 4-mercaptopyridine & 1008 & $290 \pm 40$ & $4.95 \pm 0.2$ & $8.6 \pm 0.3$ & $0.46 \pm 0.02$ \\
\hline Benzoic Acid & 1004 & $220 \pm 30$ & $6.1 \pm 0.5$ & $7.1 \pm 0.5$ & $0.0512 \pm$ \\
& 1074 & $290 \pm 40$ & $9.1 \pm 0.6$ & $\begin{array}{c}18.2 \pm \\
0.9\end{array}$ & $0.39 \pm 0.02$ \\
\hline $\begin{array}{l}\text { 4-methylthiobenzoic } \\
\text { acid }\end{array}$ & 1004 & $220 \pm 30$ & $0.21 \pm 0.02$ & $\begin{array}{c}0.30 \pm \\
0.02\end{array}$ & $0.122 \pm 0.008$ \\
\hline Phenylalanine & 1002 & $290 \pm 40$ & $0.124 \pm 0.007$ & $\begin{array}{c}0.209 \pm \\
0.005\end{array}$ & $0.091 \pm 0.005$ \\
\hline Phenylalanine-Cysteine & 1006 & $565 \pm 20$ & $0.59 \pm 0.01$ & $6.58 \pm$ & $0.0049 \pm$ \\
& & & & 0.09 & 0.0002 \\
\hline Pyridine (Au)
\end{tabular}

a Obtained using $50 \pm 10 \mathrm{~nm}$ citrate reduced silver nanoparticles

b Obtained using $50 \pm 5 \mathrm{~nm}$ citrate reduced gold nanoparticles

c Obtained using $247 \mathrm{~mW}$ excitation, 3.5 micron beam diameter

d Obtained using $70 \mathrm{~mW}$ excitation, 1.6 micron beam diameter

e Obtained using $15 \mathrm{~mW}$ excitation, 0.6 micron beam diameter 

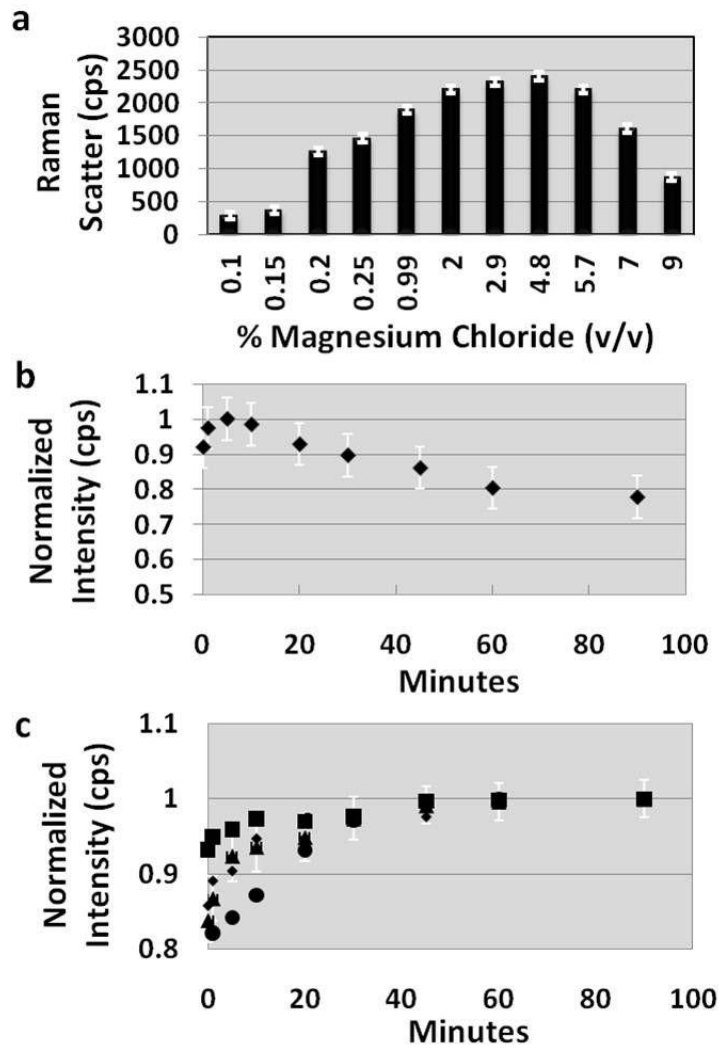

Figure 1. (a) SER intensity for pyridine's $1006 \mathrm{~cm}^{-1}$ peak using $290 \pm 40 \mathrm{~nm}$ aggregates thirty minutes after adding the indicated amount of $0.5 \mathrm{M}$ magnesium chloride; (b) normalized SER intensity for pyridine's $1006 \mathrm{~cm}^{-1}$ peak using $290 \pm 40 \mathrm{~nm}$ aggregates and $4.7 \% \mathrm{v} / \mathrm{v} 0.5 \mathrm{M}$ magnesium chloride at varying times after salt addition; and (c) same as (b) except using $0.99 \% \mathrm{v} / \mathrm{v} 0.5 \mathrm{M}$ magnesium chloride. The aggregate size was circle: $220 \pm 30$ $\mathrm{nm}$; triangle: $290 \pm 40 \mathrm{~nm}$; small square: $340 \pm 20 \mathrm{~nm}$; large square: $910 \pm 40 \mathrm{~nm}$; diamond: $960 \pm 60 \mathrm{~nm}$. Excitation wavelength $1064 \mathrm{~nm}$; excitation power $247 \mathrm{~mW} ; 10$ second integration with 3 accumulations. Error bars represent one standard deviation from three replicate measurements. 

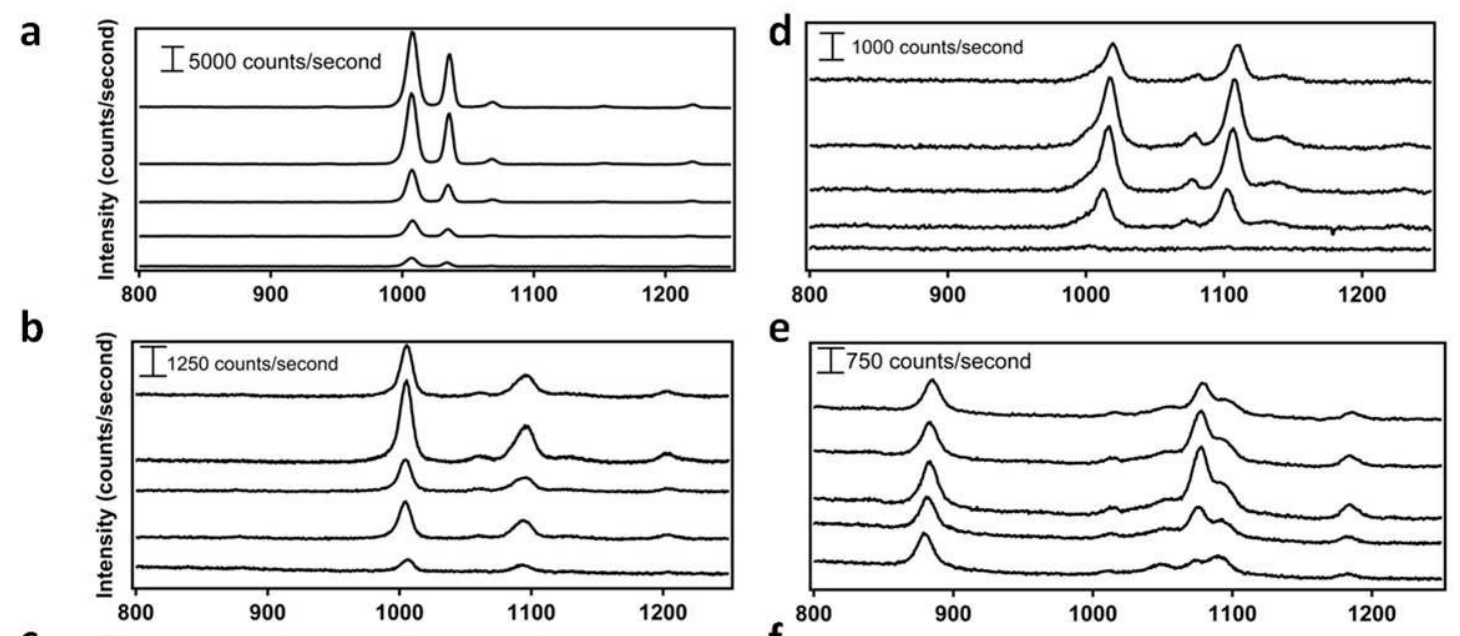

C
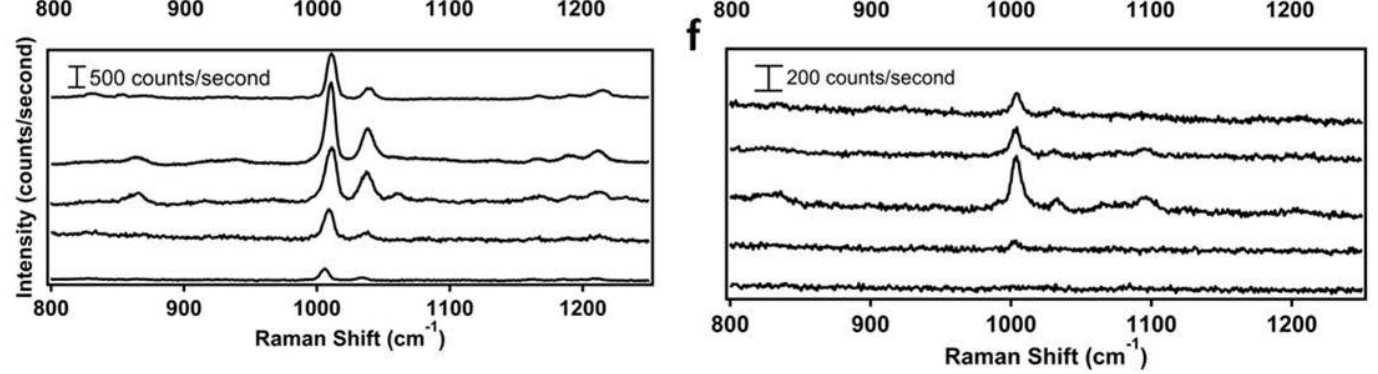

Figure 2. Surface enhanced Raman spectra using 1064 nm excitation of (a) pyridine; (b) 4mercaptopyridine; (c) benzoic acid; (d) 4-methylthiobenzoic acid; (e) phenylalanine; and (f) phenylalanine-cysteine obtained using silver particles with varying average diameters after aggregation with $0.99 \% \mathrm{v} / \mathrm{v} 0.5 \mathrm{M}$ magnesium chloride. The spectra from top to bottom were collected using silver colloids with the following average diameter (aggregate size): 40 $\pm 10(340 \pm 20) \mathrm{nm}, 50 \pm 10(220 \pm 30) \mathrm{nm}, 70 \pm 20(290 \pm 40) \mathrm{nm}, 80 \pm 20(910 \pm 40) \mathrm{nm}$ and $100 \pm 20(960 \pm 60) \mathrm{nm}$. Excitation wavelength $1064 \mathrm{~nm}$; excitation power $247 \mathrm{~mW} ; 10$ second integration with 3 accumulations. 

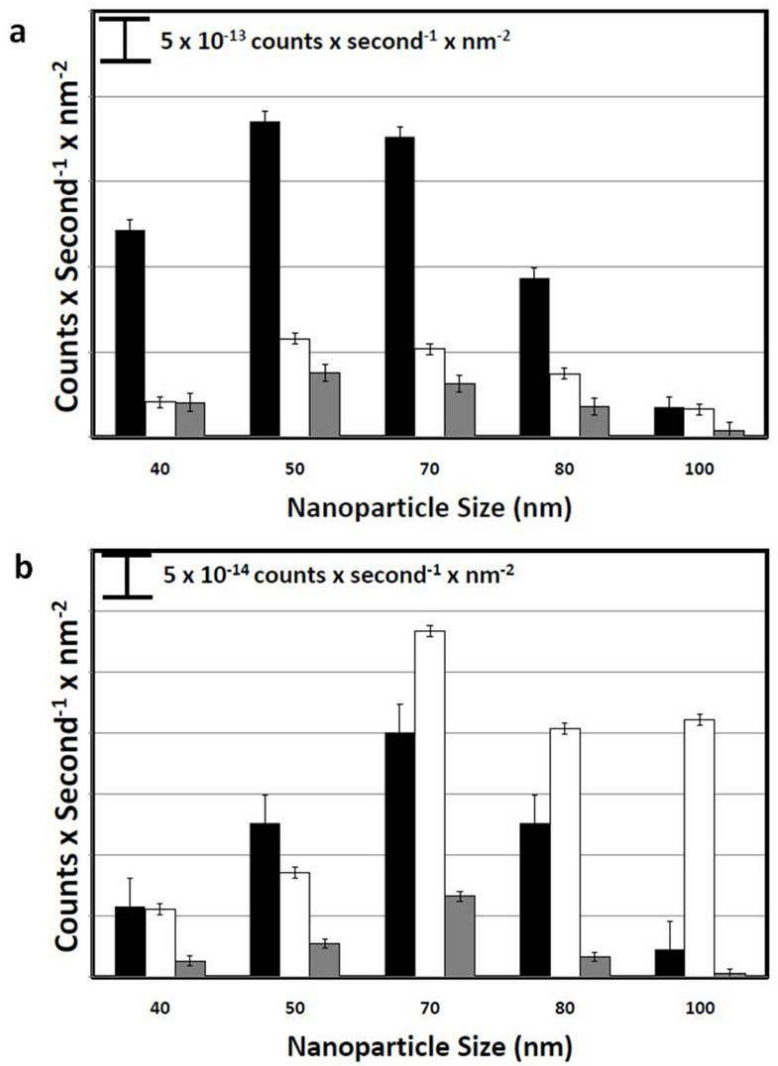

Figure 3. The nanoparticle surface area adjusted Raman peak area for the analytes shown in Figure 2. The $\mathrm{x}$-axis is the average nanoparticle diameter; the corresponding aggregate size is listed in Table 1. (a) Black: $1006 \mathrm{~cm}^{-1}$ pyridine, White: $1004 \mathrm{~cm}^{-1}$ benzoic acid, Gray: 1004 $\mathrm{cm}^{-1}$ phenylalanine obtained using $1064 \mathrm{~nm}$ excitation; (b) Black: $1008 \mathrm{~cm}^{-1} 4-$ mercaptopyridine, White: $1074 \mathrm{~cm}^{-1}$ 4-methylthiobenzoic acid, Gray: $1004 \mathrm{~cm}^{-1}$ phenylalanine-cysteine obtained using $1064 \mathrm{~nm}$ excitation. The peak used for each analyte is listed in Supplemental Information Table S1. Error bars represent one standard deviation from three replicate measurements. 
a

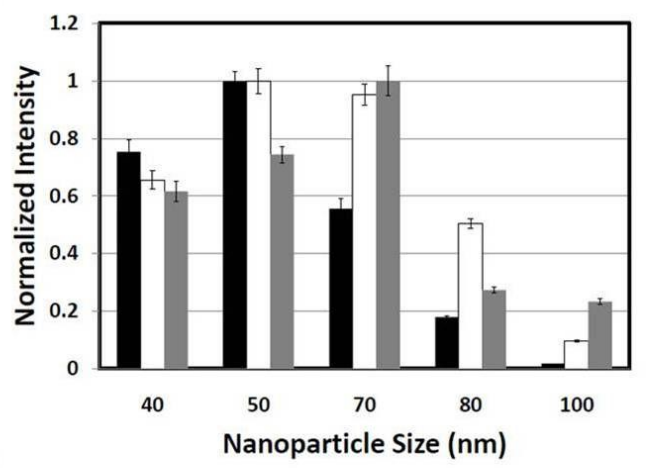

b

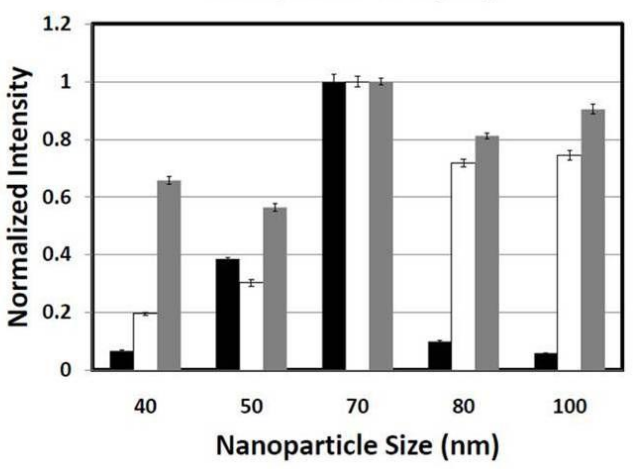

Figure 4. The nanoparticle surface area adjusted (a) $1006 \mathrm{~cm}^{-1}$ pyridine or (b) $1008 \mathrm{~cm}^{-1} 4$ mercaptopyridine Raman peak area obtained using nanoparticles with the indicated average diameter after aggregating with $0.99 \% \mathrm{v} / \mathrm{v} 0.5 \mathrm{M}$ magnesium chloride and $140 \mathrm{~mW} 785$ (black), $247 \mathrm{~mW} 1064$ (white), $15 \mathrm{~mW} 488$ (gray) nm excitation. The signal has been normalized to the highest value obtained for each wavelength and analyte. Error bars represent one standard deviation from three replicate measurements. 

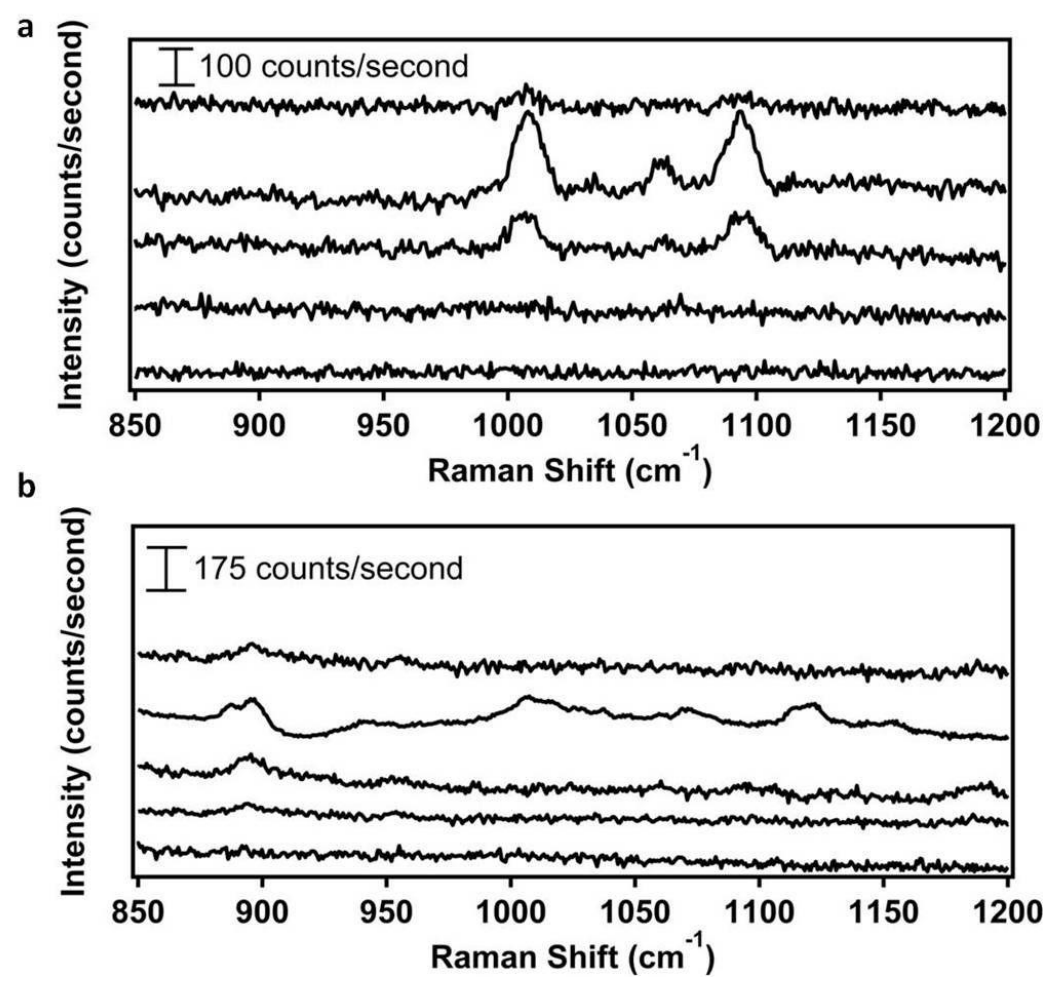

Figure 5. SER spectra of (a) angeotensin I (Asp-Arg-Val-Tyr-Val-His-Pro-Phe-His-Leu) (b) hemoglobin (95 aromatic amino acids, 2 cysteine) obtained using $1064 \mathrm{~nm}$ excitation with silver nanoparticles of varying average diameters aggregated with $0.99 \% \mathrm{v} / \mathrm{v} 0.5 \mathrm{M}$ magnesium chloride. The spectra from top to bottom were collected using silver colloids with the following average diameter (aggregate size): $40 \pm 10(340 \pm 20) \mathrm{nm}, 50 \pm 10(220 \pm$ 30) nm, $70 \pm 20(290 \pm 40) \mathrm{nm}, 80 \pm 20(910 \pm 40) \mathrm{nm}$ and $100 \pm 20(960 \pm 60)$. Excitation wavelength $1064 \mathrm{~nm}$; excitation power $247 \mathrm{~mW}$; 10 second integration with 3 accumulations. 
Supplemental Information

\begin{abstract}
Optimization of Silver Nanoparticles for Surface Enhanced Raman Spectroscopy of Structurally Diverse Analytes using Visible and Near-Infrared Excitation
\end{abstract}

Matthew W. Meyer and Emily A. Smith

Ames Laboratory, U. S. Department of Energy, Ames, Iowa 50011-3111, and Department of Chemistry, Iowa State University, Ames, Iowa 50011-3111 

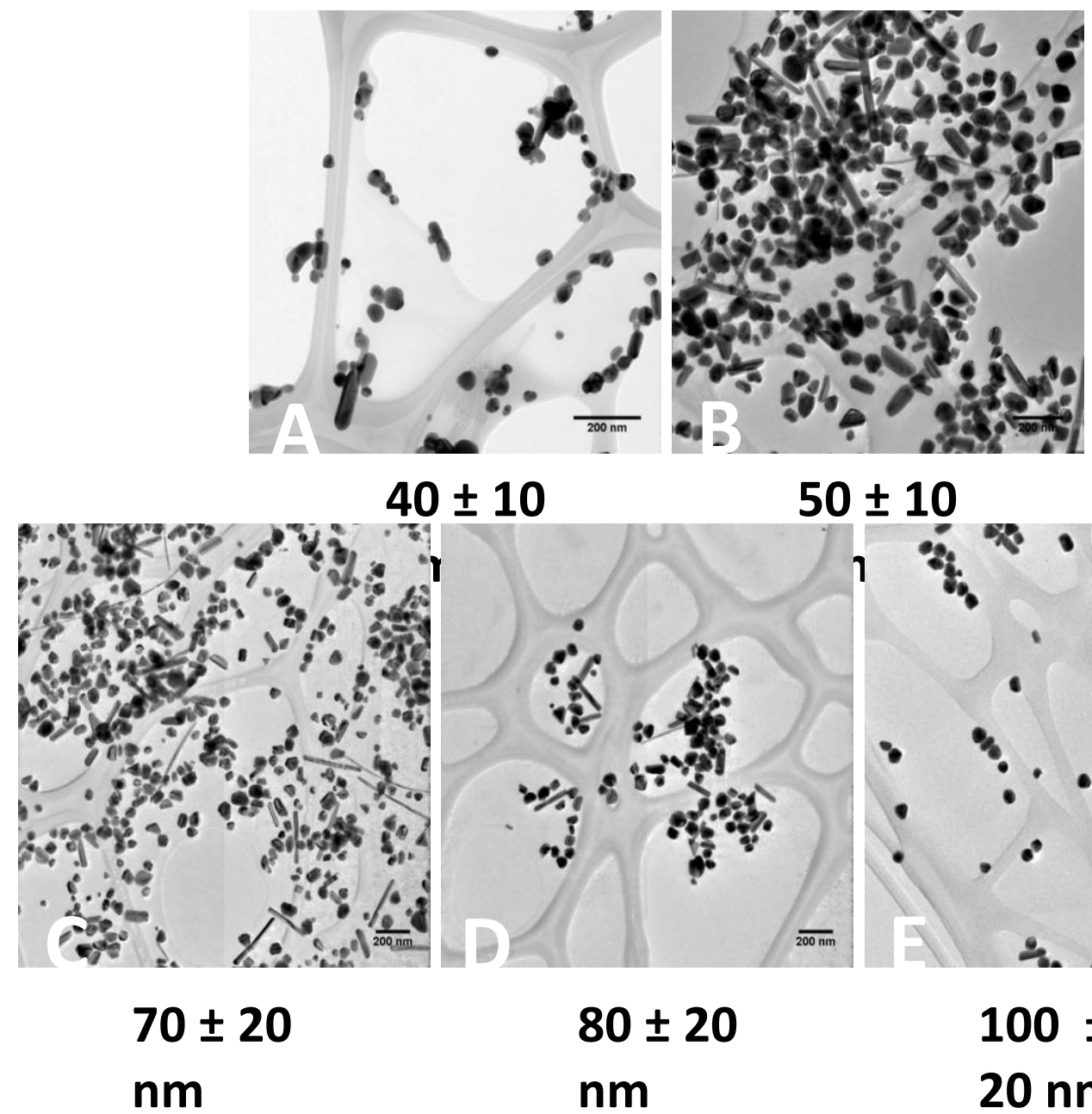

$\pm 10$

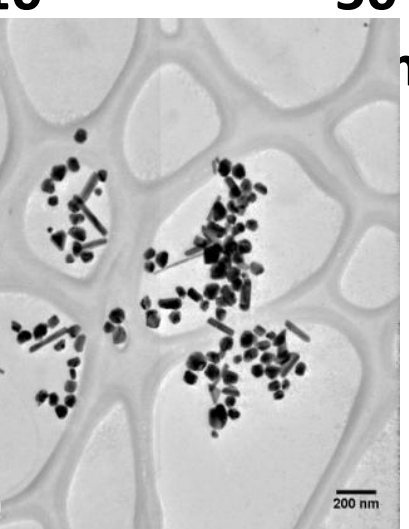

$50 \pm 10$
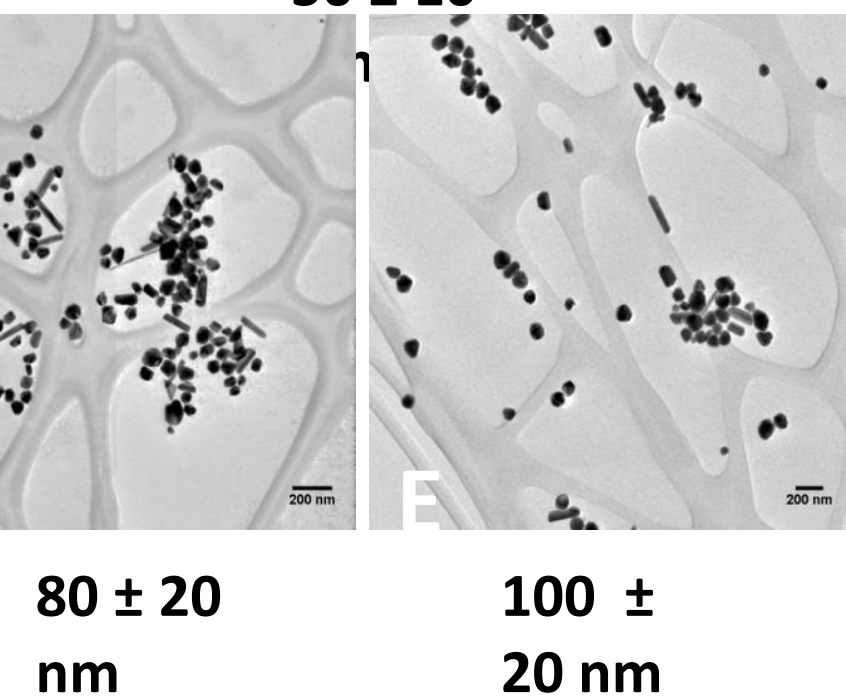

$100 \pm$ $20 \mathrm{~nm}$

Figure S1. Transmission electron microscopy images of silver nanoparticles synthesized with (a) $1.5 \mathrm{~mL}$; (b) $1.0 \mathrm{~mL}$; (c) $0.8 \mathrm{~mL}$; (d) $0.5 \mathrm{~mL}$; (e) $0.2 \mathrm{~mL} 1 \% \mathrm{w} / \mathrm{v}$ citrate. See Experimental section for additional details. 

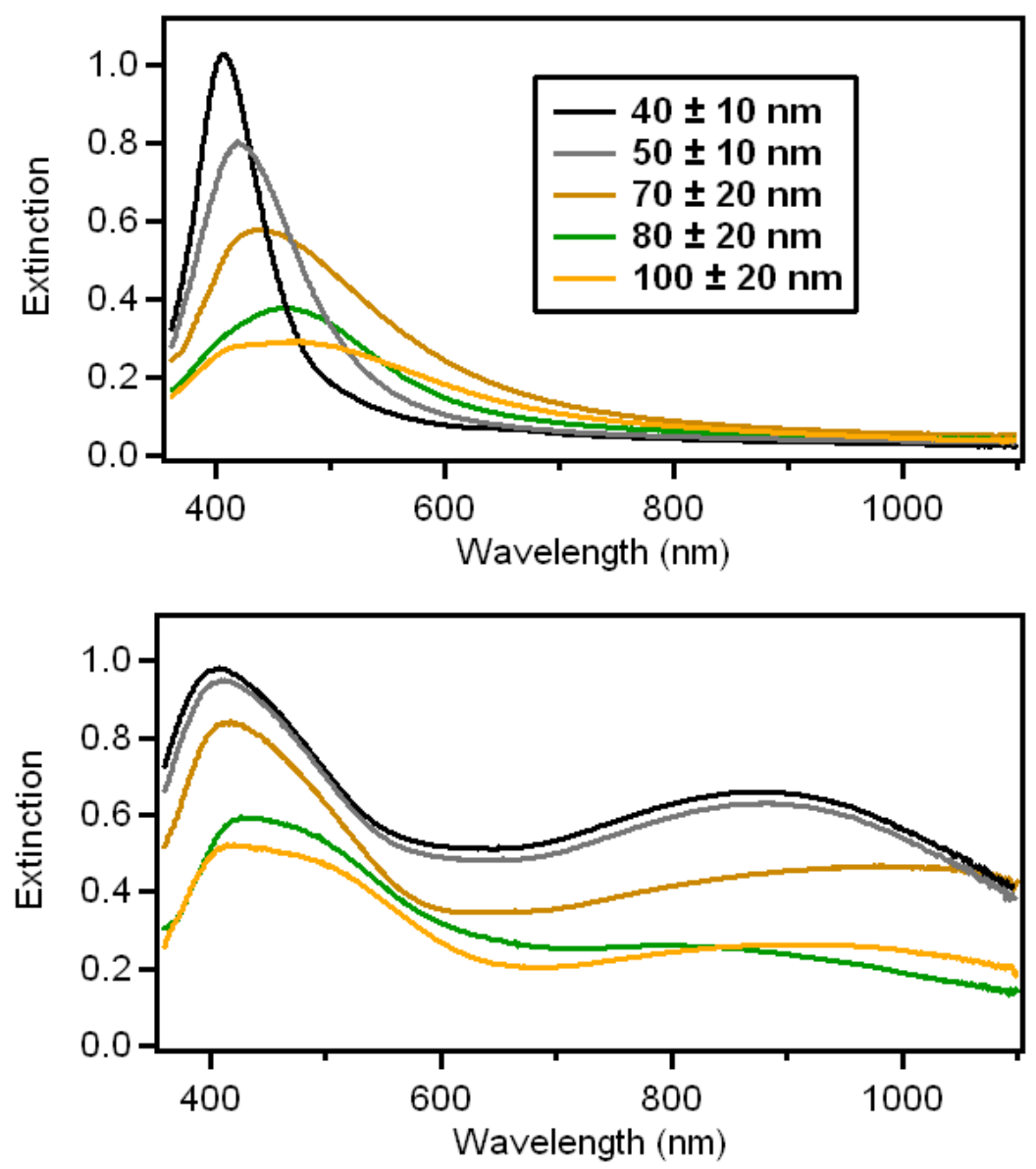

Figure S2. Extinction curves for the silver nanoparticles with the indicated average diameter (top) before and (bottom) after adding $50 \mu \mathrm{L} 2 \mathrm{mM}$ pyridine mixed with $450 \mu \mathrm{L} 70 \mathrm{~nm}$ silver nanoparticles and $5 \mu \mathrm{L} 0.5 \mathrm{M} \mathrm{MgCl}_{2}$ (final concentration $0.99 \% \mathrm{v} / \mathrm{v}$ ). The color scheme is the same in both graphs. 


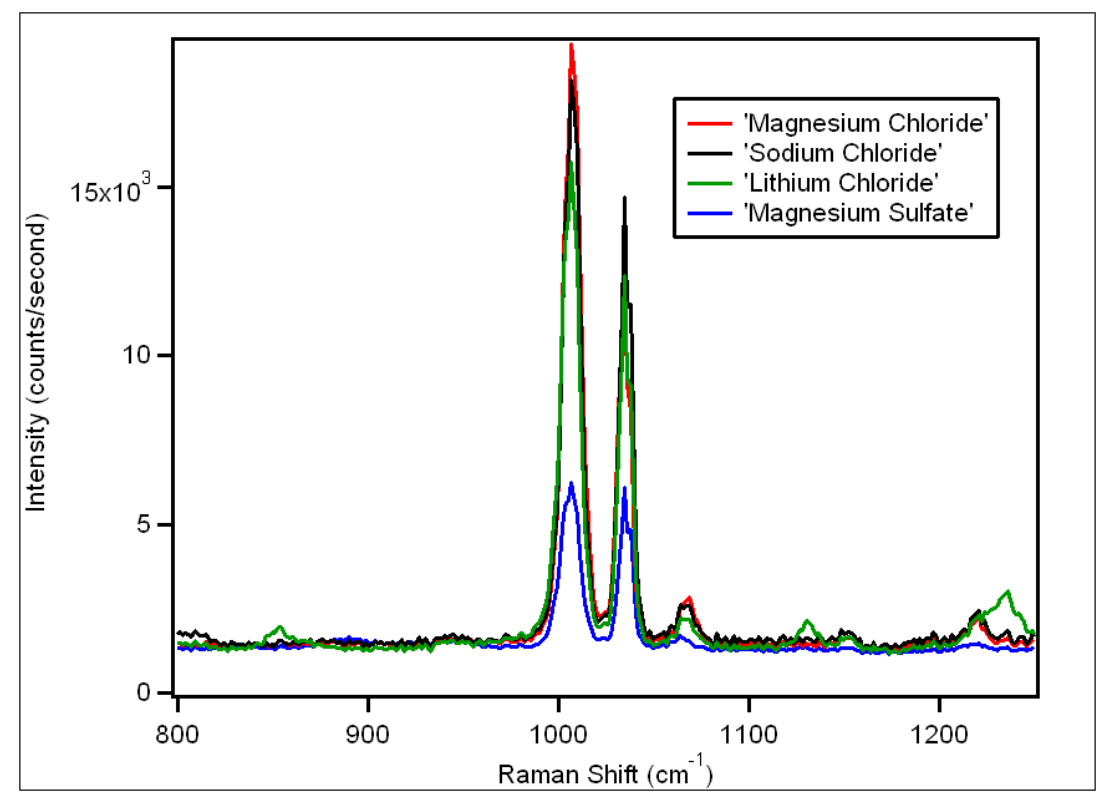

Figure S3. Surface enhanced Raman spectra of $50 \mu \mathrm{L} 2 \mathrm{mM}$ pyridine mixed with $450 \mu \mathrm{L} 70$ nm silver nanoparticles and $5 \mu \mathrm{L} 0.5 \mathrm{M}$ (final concentration $0.99 \% \mathrm{v} / \mathrm{v}$ ) of the indicated salt. 


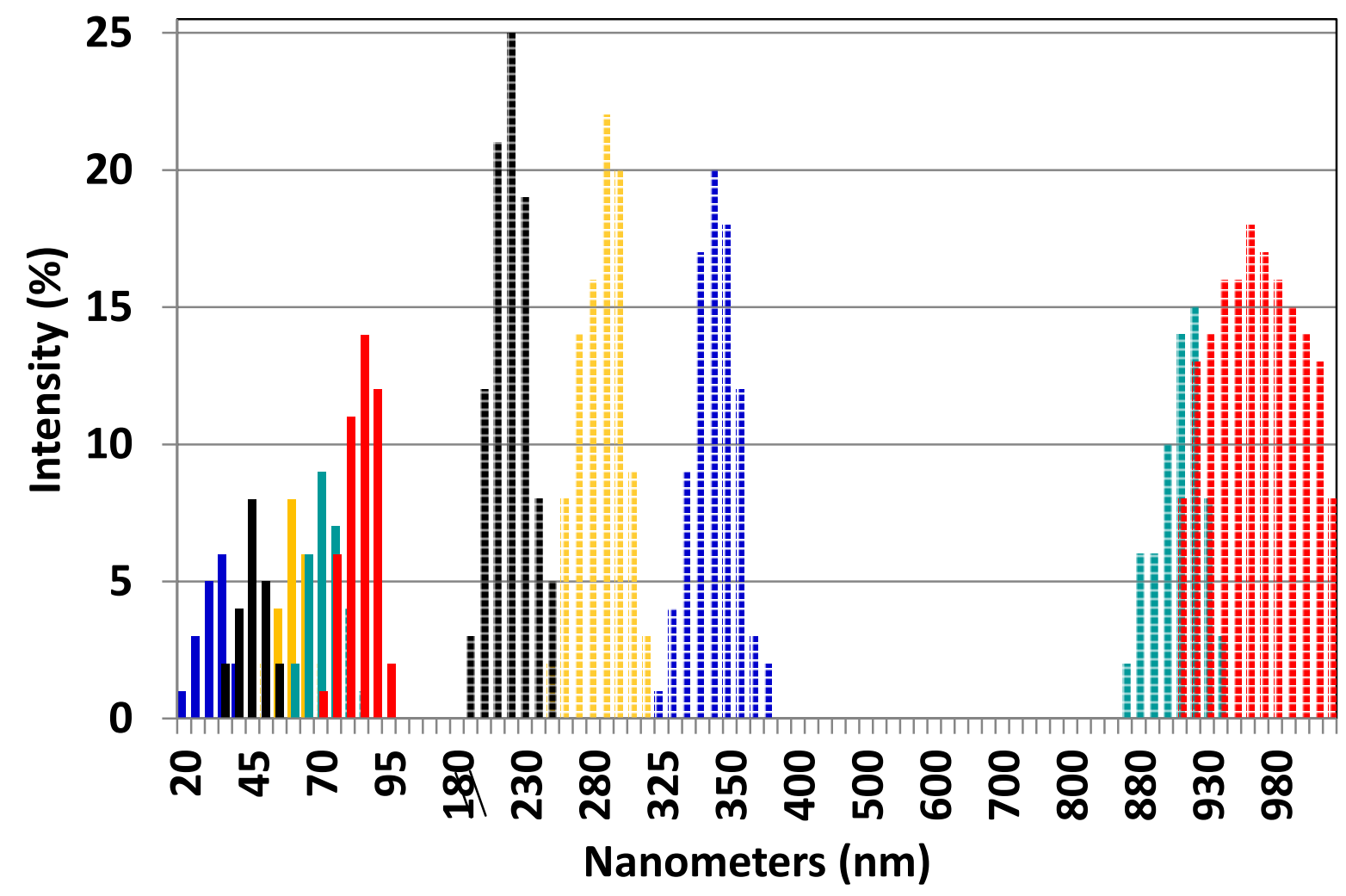

Figure S4. Histograms showing nanoparticle and aggregate hydrodynamic diameter as measured by dynamic light scattering for particles before (solid markers) and after (dashed markers) adding $0.99 \% \mathrm{v} / \mathrm{v} 0.5 \mathrm{M} \mathrm{MgCl}_{2}$. The amount of $1 \% \mathrm{w} / \mathrm{v}$ citrate added during nanoparticle synthesis was (blue) $1.5 \mathrm{~mL}$; (black) $1.0 \mathrm{~mL}$; (yellow) $0.8 \mathrm{~mL}$; (green) $0.5 \mathrm{~mL}$; (red) $0.2 \mathrm{~mL}$. The corresponding aggregates follow the same color scheme. 


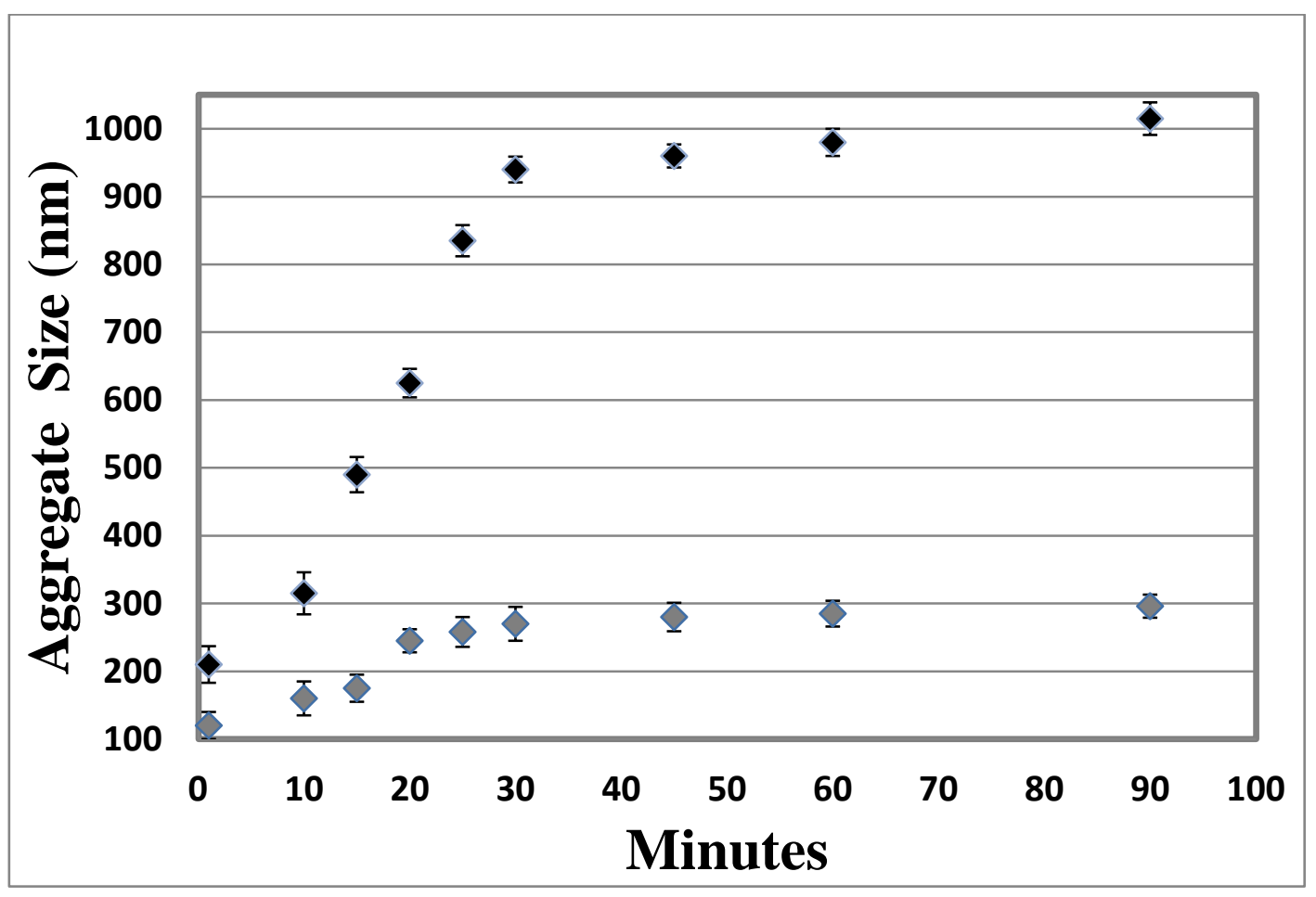

Figure S5. Average aggregate hydrodynamic diameter for $70 \pm 20 \mathrm{~nm}$ silver nanoparticles (grey) and $80 \pm 20 \mathrm{~nm}$ silver nanoparticles (black), as measured by dynamic light scattering, for the solution containing $50 \mu \mathrm{L} 2 \mathrm{mM}$ pyridine, $450 \mu \mathrm{L}$ silver nanoparticles and $5 \mu \mathrm{L} 0.5 \mathrm{M}$ $\mathrm{MgCl}_{2}$ between 1 minute to 90 minutes after adding the salt aggregating agent. Stable aggregates are measured between 30 and 90 minutes after mixing. 
A

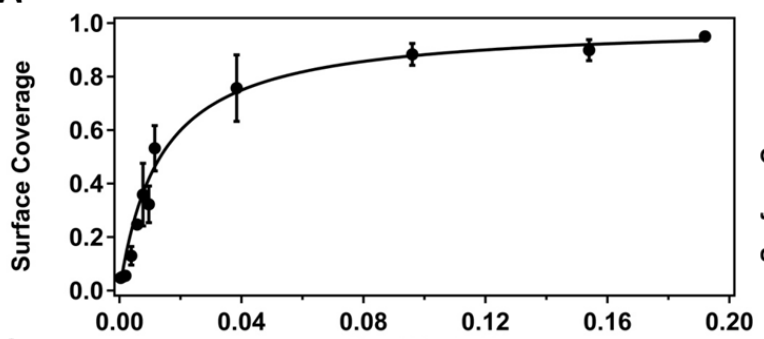

C

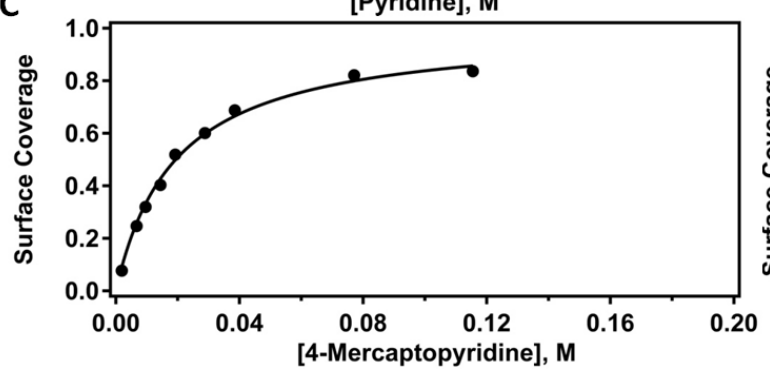

B
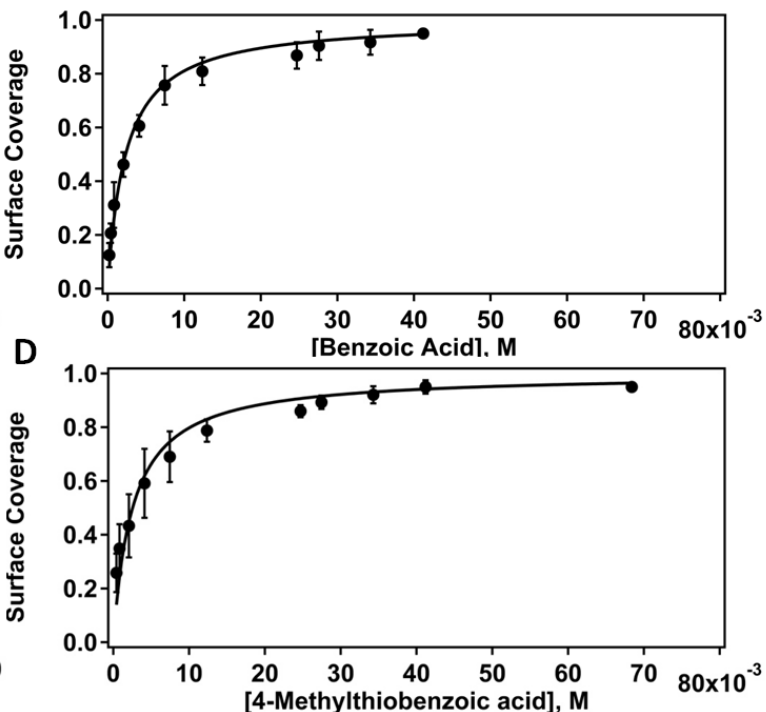

Figure S6. Adsorption isotherms measured by Raman spectroscopy for the adsorbates: (a)

pyridine; (b) benzoic acid; (c) 4-mercaptopyridine; (d) 4-methylthiobenzoic acid. Isotherms were analyzed with the $1006 \mathrm{~cm}^{-1}$ (pyridine), $1004 \mathrm{~cm}^{-1}$ (benzoic acid), $1008 \mathrm{~cm}^{-1}$ (4mercaptopyridine), and $1074 \mathrm{~cm}^{-1}$ (4-methylthiobenzoic acid) Raman peak areas. The spectral contribution of molecules in solution represent no more than $0.9 \%$ (pyridine); $3.3 \%$ (4-mercaptopyridine); $4.6 \%$ (4-methylthiobenzoic acid); or 3.6\% (benzoic acid) of the total signal at the highest analyte concentration. Data were collected using $247 \mathrm{~mW} 1064 \mathrm{~nm}$ excitation with 10 second acquisition times. The concentration of nanoparticles and salt were held constant using the concentrations described in the Experimental section: SERS measurements, while varying the concentration of analyte. The data fit well to a Langmuir isotherm model (solid line) within experimental uncertainty. Error bars represent one standard deviation from at least three replicate experiments. 
Table S1. Raman peaks used to compare intensities and surface enhancements with the indicated analytes.

\begin{tabular}{|c|c|c|c|}
\hline Molecule & $\begin{array}{l}\text { Aqueous } \\
\text { Solution }\left(\mathrm{cm}^{-1}\right)\end{array}$ & SERS $\left(\mathrm{cm}^{-1}\right)$ & Assignment \\
\hline Pyridine & 1002 & 1006 & Ring Breathing Mode \\
\hline $\begin{array}{l}\text { 4- } \\
\text { Mercaptopyridine }\end{array}$ & 1002 & 1008 & Ring Breathing Mode \\
\hline Benzoic Acid & 1004 & 1004 & Ring Breathing Mode \\
\hline $\begin{array}{l}\text { 4-Methylthio } \\
\text { Benzoic Acid }\end{array}$ & 1092 & 1074 & Ring $\mathrm{CCH}$ bending \\
\hline Phenylalanine & 1004 & 1004 & Ring Breathing Mode \\
\hline $\begin{array}{l}\text { Phenylalanine- } \\
\text { Cysteine }\end{array}$ & 1002 & 1002 & Ring Breathing Mode \\
\hline Angiotensin I & 1004 & 1004 & $\begin{array}{l}\text { Phenylalanine Ring } \\
\text { Breathing Mode }\end{array}$ \\
\hline Hemoglobin & N/A & 1125 & $\begin{array}{l}\mathrm{C}_{\mathrm{m}} \mathrm{H} \text { Out of Plane } \\
\text { Deformation Stretching } \\
\text { Mode }\end{array}$ \\
\hline
\end{tabular}




\title{
CHAPTER 6: 1064 NM DISPERSIVE MULTICHANNEL RAMAN SPECTROSCOPY FOR THE ANALYSIS OF PLANT LIGNIN
}

A paper published in Anal. Chim. Acta., 2011, 706, 164-170.

Matthew W. Meyer, Jason S. Lupoi and Emily A. Smith

Ames Laboratory, U. S. Department of Energy, Ames, Iowa 50011-3111, and Department of

Chemistry, Iowa State University, Ames, Iowa 50011-3111

\begin{abstract}
The mixed phenylpropanoid polymer lignin is one of the most abundant biopolymers on the planet and is used in the paper, pulp and biorenewable industries. For many downstream applications, the lignin monomeric composition is required, but traditional methods for performing this analysis do not necessarily represent the lignin composition as it existed in the plant. Herein, it is shown that Raman spectroscopy can be used to measure the lignin monomer composition. The use of $1064 \mathrm{~nm}$ excitation is needed for lignin analyses since high fluorescence backgrounds are measured at wavelengths as long as $785 \mathrm{~nm}$. The instrument used for these measurements is a $1064 \mathrm{~nm}$ dispersive multichannel Raman spectrometer that is suitable for applications outside of the laboratory, for example in-field or
\end{abstract}


in-line analyses and using remote sensing fiber optics. This spectrometer has the capability of acquiring toluene/acetonitrile spectra with $800 \mathrm{~cm}^{-1}$ spectral coverage, $6.5 \mathrm{~cm}^{-1}$ spectral resolution and $54 \mathrm{~S} / \mathrm{N}$ ratio in $10 \mathrm{~s}$ using $280 \mathrm{~mW}$ incident laser powers. The 1135-1350 $\mathrm{cm}^{-1}$ and $1560-1650 \mathrm{~cm}^{-1}$ regions of the lignin spectrum can be used to distinguish among the three primary model lignin monomers: coumaric, ferulic and sinapic acids. Mixtures of the three model monomers and first derivative spectra or partial least squares analysis of the phenyl ring breathing modes around $1600 \mathrm{~cm}^{-1}$ are used to determine sugarcane lignin monomer composition. Lignin extracted from sugarcane is shown to have a predominant dimethoxylated and monomethoxylated phenylpropanoid content with a lesser amount of non-methoxylated phenol, which is consistent with sugarcane's classification as a non-woody angiosperm. The location of the phenyl ring breathing mode peaks do not shift in ethanol, methanol, isopropanol, 1,4 dioxane or acetone.

\section{INTRODUCTION}

Lignin is one of three primary biopolymers in plant cell walls $[1,2]$. It is a heteropolymer composed of phenylpropanoid monomers with the aromatic portions: phydroxyphenyl, guaiacyl, and syringyl. These monomers are often referred to by the one letter designation $\mathrm{H}, \mathrm{G}$, and $\mathrm{S}$, respectively. Lignin is classified as heterogeneous not only because of its complex composition, but also because lignin from different species, tissue types, plant age and growing conditions can be quite different. Lignin is classified as predominately G (gymnosperms), G/S (angiosperms) and G/S/H (non-woody angiosperms), although many exceptions to these classifications are known [3-5]. Lignin has many 
commercial uses in the pulp and paper industry and as the raw material for biorenewable fuels and commodity chemicals. For these applications it is desirable to know the lignin purity and composition. For example, the resulting complex chemical mixture generated from lignin pyrolysis (i.e., bio-oil) is dependent on the starting monomer composition [6]. Additionally, the degradation of lignin in the environment is slow, and the monomer composition is a biomarker that can be used to identify the source of sediments and soils containing decaying plant materials [6]. Reported lignin monomer compositions in the literature have large variations due to the commonly utilized extraction and analysis procedures, which can alter phenylpropanoid structure [5, 7-9].

Raman spectroscopy has been used to measure chemical content in a wide-range of samples [10-14]. The appropriate selection of excitation wavelength is one of the most important factors for Raman spectroscopy experiments. Near infrared (commonly 785 to $1064 \mathrm{~nm}$ ) excitation is often used for detecting biological materials that can exhibit fluorescence backgrounds using visible wavelengths [15]. The intensity of Raman scatter is proportional to the incident frequency to the fourth power. Using the same laser radiance, excitation with $785 \mathrm{~nm}$ will generate 3.8-times more scattered photons compared to $1064 \mathrm{~nm}$ excitation. Despite the low energy of the photons, and even with low fluorescence quantum yield samples, a fluorescence background may render the Raman spectrum undetectable or increase detection limits using $785 \mathrm{~nm}$ excitation [16]. In these cases, the lower background obtained with $1064 \mathrm{~nm}$ excitation often outweighs the reduced scattering intensity [17]; and higher laser radiances may be used to compensate for the lower Raman cross section above one micron. The use of up to $2.3 \mathrm{~W}$ incident $1064 \mathrm{~nm}$ laser powers have been reported in the literature for Raman measurements [16, 18]. 
Reports of multichannel dispersive $1064 \mathrm{~nm}$ Raman spectrometers date back to 1986 [19], yet only a handful of references using a dispersive $1064 \mathrm{~nm}$ instrument are found in the recent literature. Fourier Transform (FT) Raman spectroscopy remains the primary instrument format for obtaining fluorescence-free Raman spectra. Yet, dispersive systems are better suited for many applications where robust, low cost instruments and remote sensing fibers are desirable [20-22]. Recently, portable Raman spectrometers have been made commercially available [23]. However, many of these systems are limited in performance characteristics, such as spectral range and spectral resolution.

The main limitation of $1064 \mathrm{~nm}$ dispersive Raman spectrometers is the availability of suitable low-noise detectors. Single channel detectors require minutes to hours to collect a 1064 nm Raman spectrum [18, 24-26]. Multichannel dispersive Raman instruments with excitation wavelengths below $800 \mathrm{~nm}$ commonly utilize charge coupled devices (CCD). CCDs have a low response beyond $1.1 \mu \mathrm{m}$, limiting their use in $1064 \mathrm{~nm}$ Raman spectroscopy [27]. Hamaguchi et al. overcame this limitation using a transfer electron InPInGaAs photocathode image intensifier CCD for dispersive $1064 \mathrm{~nm}$ Raman spectroscopy for the analysis of DNA and lung tissue $[16,26,28]$. This detector has a $\sim 4 \%$ quantum efficiency between 1 and 1.4 microns ( 0 to $2250 \mathrm{~cm}^{-1}$ Raman shift) and required lengthy acquisition times. CCDs have also been used to collect dispersive $1064 \mathrm{~nm}$ excitation antistokes Raman spectra [29].

Indium gallium arsenide (InGaAs) detectors with quantum efficiencies above $80 \%$ have high sensitivity between 900 and $1,700 \mathrm{~nm}$. This enables detection out to the $3,100 \mathrm{~cm}^{-}$ ${ }^{1} \mathrm{C}-\mathrm{H}$ stretching region using $1064 \mathrm{~nm}$ excitation. These quantum efficiencies are substantially higher than those of the competing germanium detectors [30]. Dispersive 144 
multichannel $1064 \mathrm{~nm}$ Raman spectrometers equipped with 128 or 256 element Ge or InGaAs arrays have been used to measure strong Raman scattering solids and liquids (e.g., toluene, anthracene) [26, 31-33]. These multichannel InGaAs detectors had high dark and read noise and provide limited $100-500 \mathrm{~cm}^{-1}$ spectral coverage in a single acquisition. The development of improved multichannel InGaAs detectors with 1024 or more elements and low read noise has opened the possibility of improved $1064 \mathrm{~nm}$ dispersive multichannel Raman instruments with wide spectral coverage that compete with FT Raman instruments.

Raman spectroscopy is an ideal non-invasive screening method to measure lignin composition. Lignin has been previously analyzed by Kerr gated resonance Raman spectroscopy using a benzoquinone/laccase/mediator treatment to generate a charge transfer state that extends lignin's absorption out to $500 \mathrm{~nm}$ [34]. Ultraviolet RR spectroscopy has also been used to measure lignin since the fluorescence signal is spectrally separated from the Raman spectrum at these wavelengths [35]. FT Raman and surface enhance FT Raman spectroscopies using a $600 \mathrm{~mW} 1064 \mathrm{~nm}$ laser have been used to measure and assign lignin spectral peaks [36]. The presence of lignin in plant tissues has also been measured by Raman spectroscopy [37, 38]. Finally, the spectral differences of three model lignin monomers were identified experimentally and using DFT calculations [39], but were not extended to real plant lignin samples or monomer mixtures. The reported differences in the monomer spectra reveal the possibility of measuring lignin compositions using Raman spectroscopy.

Herein, we describe a dispersive 1024-multichannel $1064 \mathrm{~nm}$ Raman spectrometer with wide spectral coverage, S/N values that exceed those of a FT Raman instrument for most acquisition times, and background values orders of magnitude lower than a dispersive $785 \mathrm{~nm}$ Raman instrument for the analysis of lignin and model lignin monomers. The results 
show that the new generation multichannel InGaAs array detectors produce high quality spectra with $\sim 280 \mathrm{~mW}$ laser radiance using relatively fast acquisition times, and will enable facile measurements in laboratory and other settings. It is demonstrated that Raman spectroscopy can be used to quantitatively measure the lignin monomer composition using partial least squares analysis and qualitatively using first derivative spectra.

\section{EXPERIMENTAL}

Materials

All chemicals were of analytical grade and purchased from Sigma-Aldrich (St. Louis, MO). Hydrolytic lignin (Aldrich, CAS 8072-93-3) was extracted from sugarcane. Aqueous

solutions were prepared using deionized water $\left(18.2 \mathrm{M} \Omega \mathrm{cm}^{-1}\right)$ from an Ultrapure II water system (Thermo Scientific, Waltham, MA).

Dispersive Multichannel $1064 \mathrm{~nm}$ Instrumentation

A home-built 1064 nm dispersive multichannel Raman instrument is shown in Figure 1. A $1064 \mathrm{~nm} \mathrm{Nd:YVO} 4$ solid state laser (Blue Sky Research, Milpitas, CA) with a maximum output power of $1 \mathrm{~W}$ was used as the excitation source. The beam was focused at the sample with a plano-convex lens with a focal length of $250 \mathrm{~mm}$ (Newport Optics, Irvine, CA). Liquid samples were placed in a nuclear magnetic resonance tube (Sigma-Aldrich), and mounted on a home-built sample stage. Raman scatter was collected in a 90 degree collection geometry. The light was collected with a plano-convex lens with a focal length of $60 \mathrm{~mm}(\mathrm{f} / 1.2)$. The light was focused with a plano-convex lens with a focal length of 200 $\mathrm{mm}(\mathrm{f} / 3.9)$. The optical system was designed to slightly overfill the $150 \mu \mathrm{m}$ entrance slit of 
the spectrometer to maximize signal. A holographic super notch filter (HNF) centered at 1064 nm (Kaiser Optical Systems, Ann Arbor, MI) was placed before the spectrometer. To aid in laser alignment, a helium neon laser was made co-linear with the $1064 \mathrm{~nm}$ laser using a dichroic mirror (Omega Optical, Brattleboro, VT) to reflect the $633 \mathrm{~nm}$ light and transmit the $1064 \mathrm{~nm}$ light. The collected signal was sent to a iHR320 spectrometer, fitted with a 1024 element InGaAs detector (Horiba Jobin Yvon, Edison, NJ). The InGaAs detector had a read noise of $462.1 \mathrm{e}^{-} \mathrm{rms}$ and a dark current of $3.7 \mathrm{ke}^{-}$pixel $^{-1}$ second $^{-1}$ when cooled to $170 \mathrm{~K}$. Among the 300, 600 grooves/mm classically ruled and 950 grooves/mm blazed holographic gratings in the spectrometer, the 600 groove $/ \mathrm{mm}$ classically ruled grating provided the highest efficiency and the best trade-off between spectral coverage and spectral resolution. Spectra were collected with $280 \mathrm{~mW}$ incident laser power, which produces less than $0.8 \pm 0.1$ ${ }^{\circ} \mathrm{C}$ sample heating in 30 minutes for aqueous samples. Other instrument details are listed in Supporting Information Table S1.

\section{Other Instrumentation}

A home-built $785 \mathrm{~nm}$ Raman microscope has been previously reported [40]. Briefly, a $785 \mathrm{~nm}$ diode laser was directed onto the rear port of a Nikon inverted microscope with a 40x magnification, 1.0 numerical aperture objective. The collected signal was sent to a Holospec VPH spectrometer (Kaiser Optical System, Ann Arbor, MI) and a near IR enhanced PIXIS CCD camera (Princeton Instruments, Trenton, NJ). FT Raman measurements were collected with a commercially available Nicolet NXR 9650 FT Raman spectrometer (Thermo Scientific).

Raman Measurements 
Spectra of a 1:1 (v/v) acetonitrile/toluene mixture were used both for wavelength calibration and to optimize the intensity of the collected Raman scatter. Laser powers were $189 \mathrm{~mW}(785 \mathrm{~nm}), 280 \mathrm{~mW}(1064 \mathrm{~nm}$ dispersive) and $480 \mathrm{~mW}(1064 \mathrm{~nm} \mathrm{FT})$. The slit widths were set to 100 micron $(785 \mathrm{~nm}$ ) and 150 micron (dispersive $1064 \mathrm{~nm}$ ). Model lignin monomer or mixture $\left(100 \mathrm{mg} \mathrm{mL}^{-1}\right)$ measurements were obtained with 60 second integrations and 3 accumulations (1064 nm dispersive) or 300 scans (1064 nm FT). Lignin $\left(50 \mathrm{mg} \mathrm{mL}^{-1}\right)$ measurements were acquired with 600 second integrations and 3 accumulations using the $1064 \mathrm{~nm}$ dispersive instrument. Sinapic and ferulic acid in 1,4-dioxane or methanol were heated to $37{ }^{\circ} \mathrm{C}$ in a water bath to ensure dissolution. All Raman measurements were acquired at room temperature.

\section{Data Analysis}

Raman spectra were collected using the software Winspec32 (Princeton Instruments) for the $785 \mathrm{~nm}$ dispersive instrument and SynerJY (Horiba Jobin Yvon) for the $1064 \mathrm{~nm}$ dispersive instrument. FT Raman measurements were collected using the software Omnic (Thermo Scientific). All spectra were imported into Grams AI V 8.0 (Thermo Scientific) and background subtracted using a blank containing all matrix components except the analyte. The spectra were not intensity corrected for the instrument's varying throughput and quantum efficiency across the spectral region. Intensity correction of the spectra from the $1064 \mathrm{~nm}$ dispersive instrument can be performed using the curve shown in Supplemental Information Figure S1.

The spectra were imported into Igor Pro V 6.1 (WaveMetrics Inc. Lake Oswego, OR) for further analysis. The raw spectral intensity was divided by the total integration time to plot the spectra in counts per second. Signal-to-noise $(\mathrm{S} / \mathrm{N})$ ratios were calculated using the 
background subtracted peak intensity for the peak with highest intensity in the spectrum divided by the root mean square noise measured from 940 to $980 \mathrm{~cm}^{-1}$. The multipeak fitting package was used to deconvolute three overlapping spectral peaks in the 1560 to $1650 \mathrm{~cm}^{-1}$ region. The procedure minimizes the residual between the experimental values and the fit peaks' location, width and height. The peak shape was set to a Gaussian profile. Igor Pro V6.1 was also used to obtain first derivative spectra.

The partial least squares (PLS) analysis was performed using The Unscrambler X (Camo Inc., Oslo, Norway). A data set was comprised of 36 rows, representing 36 standard monomer mixtures, and 261 columns, representing the spectral regions of interest (1138$1231 \mathrm{~cm}^{-1}, 1257-1286 \mathrm{~cm}^{-1}, 1325-1350 \mathrm{~cm}^{-1}$, and $\left.1580-1614 \mathrm{~cm}^{-1}\right)$. The data was meancentered, and the model was randomly cross-validated. The NIPALS algorithm was used to calculate PLS factors. This model was used to predict the score coordinates for randomly selected monomer mixtures in order to test the model, and to determine the monomer composition of lignin extracted from sugarcane.

\section{RESULTS AND DISCUSSION}

\section{Comparison of 1064 and $785 \mathrm{~nm}$ Excitation for the Analysis of Lignin}

The goal of this work is to demonstrate the development and use of a $1064 \mathrm{~nm}$ dispersive multichannel Raman spectrometer for the analysis of the heterogeneous biopolymer lignin, and to show that it can be used to measure lignin monomer composition. The impact of this work will be the capability to take Raman measurements in diverse 
locations using robust instrumentation and the identification of lignin compositions using Raman spectra for several downstream applications.

In order to determine the most suitable wavelength for the analysis of lignin, the Raman spectra of an extracted lignin sample were collected using 785 and $1064 \mathrm{~nm}$ excitation (Figure 2A). Since the Raman scatter scales with the excitation frequency to the fourth power, the highest possible laser frequency that produces minimal fluorescence background should be used. The $785 \mathrm{~nm}$ spectrum with 60 second integration and 3 accumulations shows no discernible Raman peaks and a broad background that is 160 times higher than the background of the $1064 \mathrm{~nm}$ Raman spectrum with 600 second integration and 3 accumulations. The fluorescence spectrum of the same lignin sample, when excited with $785 \mathrm{~nm}$, shows a low intensity peak that mimics the $785 \mathrm{~nm}$ Raman spectrum background (Supplemental Information Figure S2). This fluorescence is either intrinsic to the native lignin or a result of the extraction process.

The low energy photons of the $1064 \mathrm{~nm}$ laser produce a flat background in the Raman spectrum and discernible peaks that are characteristic of lignin (Figure 2B). The peak assignments are shown in Supplemental Information Table S2. The most intense peaks are centered around $\sim 1600 \mathrm{~cm}^{-1}$ and are assigned to the aromatic ring breathing modes (Wilson notation $\mathrm{v}_{8 \mathrm{a}}$ and $\mathrm{v}_{8 \mathrm{~b}}$ ) and substituent carbon-carbon double bonds. The results show that $1064 \mathrm{~nm}$ excitation produces Raman spectra of extracted plant lignin with a low background.

\section{4-Channel Dispersive 1064 nm Raman Spectrometer}

The multichannel dispersive $1064 \mathrm{~nm}$ Raman spectrometer (Figure 1) leverages recent developments in InGaAs detectors with 1024 sensing elements that are 25 x 250 
microns with relatively low read and dark noise compared to previous generation near IR detectors. The $\mathrm{S} / \mathrm{N}$ ratios and spectral resolution of the $1064 \mathrm{~nm}$ dispersive Raman spectrometer are compared to a commercial FT Raman instrument using a series of tolueneacetonitrile spectra (Supplemental Information Figure S3). The S/N values were calculated and graphed in Figure 3 as a function of total analysis time, which accounts for the amount of time required to average multiple spectra when appropriate. The FT Raman S/N curve is fairly constant at all analysis times included in this study. The dispersive instrument's $\mathrm{S} / \mathrm{N}$ has been measured for: varying integration times with 1 accumulation, and set integration times (60 or 150 seconds) with varying number of accumulations. The dispersive $\mathrm{S} / \mathrm{N}$ data are independently fit to a curve with a dependence on the square root of the total analysis time. All three fit curves nearly overlap, and are consistent with a detector that is dark noise limited [30]. At total analysis times below 15 seconds, the FT spectra show a 2 to 8 -fold higher $\mathrm{S} / \mathrm{N}$ ratio compared to the dispersive instrument. However, at total analysis times greater than 15 seconds, the dispersive instrument has a higher $\mathrm{S} / \mathrm{N}$ ratio than the FT instrument. The spectral resolution is $6.5 \mathrm{~cm}^{-1}$ for the dispersive spectra and $8 \mathrm{~cm}^{-1}$ for the FT spectra, although higher spectral resolution is possible with both instruments.

In order to investigate the possibility of using Raman spectroscopy to measure the source of extracted lignin and materials derived from lignin, model phenylpropanoid monomers were studied. Lignin is primarily composed of three phenyl monomers with hydroxy- and methoxy- substituents. Three carboxylic acids were used as model lignin monomers: ferulic (guaiacyl, G); coumaric (p-hydroxyphenyl, H) and sinapic (syringyl, S) acids. The Raman spectra of the model lignin monomers obtained using both the $1064 \mathrm{~nm}$ dispersive and FT Raman spectrometers are shown in Figure 4. Under the conditions used in 
this study, similar spectra are observed with both instruments and differences in the monomer spectra are identifiable in the 1135 to $1350 \mathrm{~cm}^{-1}$ and 1560 to $1650 \mathrm{~cm}^{-1}$ spectral regions. Peaks at $1155.2 \pm 0.6$ and $1169.3 \pm 0.4 \mathrm{~cm}^{-1}$ are characteristic of sinapic and coumaric acid, respectively. The dispersive spectra shown in Figure 4 were collected without tiling together different spectral regions, and demonstrate that the dispersive Raman spectra have sufficient spectral coverage to encompass both of the critical regions for lignin monomer identification. This has been problematic with previously reported $1064 \mathrm{~nm}$ dispersive multichannel Raman instruments [31].

The phenyl ring breathing modes at $\sim 1600 \mathrm{~cm}^{-1}$ are composed of two partially resolved peaks in coumaric and ferulic acids. Both vibrations are degenerate in sinapic acid. A procedure for determining peak maxima when there is peak overlap is to use the zero crossing points in first derivative spectra (Figure 4, inset). There are identifiable peak maxima for all three monomers $(1587.8 \pm 0.4 \mathrm{H}, 1591.0 \pm 0.5 \mathrm{G}, 1594.0 \pm 0.2 \mathrm{~S}, 1603.0 \pm$ $\left.0.9 \mathrm{G}, 1606.2 \pm 0.5 \mathrm{H} \mathrm{cm}^{-1}\right)$. The additive intensity of the $\mathrm{v}_{8 \mathrm{a}}$ and $\mathrm{v}_{8 \mathrm{~b}}$ ring breathing mode peaks is sensitive to substitution for many phenyl compounds [41]. The intensity of the two peaks increases with increasing conjugation (i.e., benzene to styrene) and to a lesser extent with electron donating or electron withdrawing substituents on the phenyl ring. Peak deconvolution was used to determine the peak maxima and intensities in the 1560 to 1650 $\mathrm{cm}^{-1}$ spectral region for all three model lignin monomers (Supplemental Information Table $\mathrm{S} 3$ ). The intensity corrected [42] additive $\mathrm{v}_{8 \mathrm{a}}$ and $\mathrm{v}_{8 \mathrm{~b}}$ normalized peak intensities for the three monomers are 2.2 for $\mathrm{G}, 1.0$ for $\mathrm{S}$, and 3.5 for $\mathrm{H}$. The multipeak fitting and first derivative spectral results suggest it may be possible to evaluate the lignin monomer composition using Raman spectroscopy. 
Analysis of Lignin Monomer Composition with First Derivative Spectra and Chemometrics

The Raman spectrum of a commercially obtained lignin sample from the non-woody angiosperm sugarcane is plotted in Figure 5 along with the spectra of three model monomer mixtures with different compositions (G-S-H\%: 5-90-5, 51-40-9 and 25-25-50). Visual inspection of the spectra indicates that the lignin most closely matches the 51-40-9 model lignin monomer mixture, which is consistent with a non-woody angiosperm. There is a peak maximum at $\sim 1606 \mathrm{~cm}^{-1}$ in both the monomer mixture and lignin spectra. The peak at 1633 $\mathrm{cm}^{-1}$ is assigned to substituent carbon-carbon double bonds, and it has a lower intensity in the lignin spectrum than is measured for the model lignin monomers. This suggests that the phenyl ring's substituent aromaticity is lower in the lignin sample than the model monomers. This is not surprising since the model monomers were chosen solely for the substitution pattern of the hydroxyl- and methoxy-groups.

In order to develop a quantitative model for the lignin monomer composition, partial least squares analysis was performed on the spectra from 36 monomer mixtures (Figure 6A). The PLS scores plot forms a triangle with the pure monomers at the corners. Mixtures with high $\mathrm{G}$ content are at the top of the triangle, mixtures high in $\mathrm{S}$ are to the left and those high in $\mathrm{H}$ are at the right side of the triangle. The majority of the monomer compositions were chosen based on real plant compositions. Since H content is not high in most plants, there are fewer mixtures represented in this part of the scores plot. Using the developed model, the sugarcane hydrolytic lignin was located on the scores plot closest to the standards with the following composition (G-S-H\%): 51-40-9 and 50-25-25. This is consistent with the visual 
inspection of the Raman spectrum (Figure 5) and also for a non-woody angiosperm. For sugarcane lignin, the PLS model reported a 33-41-33 monomer composition. The uncertainty is as large as the measurement due to noise in the lignin spectrum. Higher laser powers could be used without sample damage to increase the $\mathrm{S} / \mathrm{N}$ ratio. A referenced literature value for sugarcane lignin composition is 39-33-28, which was obtained using alkaline nitrobenzene oxidation-gas chromatography.[43]

The first derivative Raman spectra of the 36 monomer mixtures were analyzed as a qualitative method for describing the monomer composition (Figure 6B). The spectra have been color coded based on the grouping shown in Figure 6A. It can be seen that the location of the monomer mixture on the scores plot corresponds to the peak maxima located using first derivative spectra. When $\mathrm{S}$ is greater than $70 \%$ of the monomer composition, there is a single peak maximum for the phenyl ring breathing mode at $1593.8 \pm 0.4 \mathrm{~cm}^{-1}$ and a second, smaller peak at $1155.0 \pm 0.9 \mathrm{~cm}^{-1}$. When $\mathrm{G}$ or $\mathrm{S} / \mathrm{G}$ dominates the monomer composition there is a peak maximum at $1603 \pm 1 \mathrm{~cm}^{-1}$ when $\mathrm{H}$ is less than $20 \%$, and $1604.7 \pm 0.3 \mathrm{~cm}^{-1}$ when $\mathrm{H}$ is between 21 and $30 \%$ of the monomer mixture. When all three monomers are present at roughly equal proportions, there is a peak maximum at $1605.8 \pm 0.2 \mathrm{~cm}^{-1}$ and when $\mathrm{H}$ is greater than $25 \%$ there is a small peak at $1168.4 \pm 0.7 \mathrm{~cm}^{-1}$. The first derivative spectra of the monomer compositions 49-51-0, 56-40-4 and 19-55-26 showed characteristics in between mixtures with predominant $\mathrm{G}$ or $\mathrm{S}$ compositions, and a clear assignment into one of the groups was not possible. The above information enables the lignin monomer composition to be qualitatively determined for most mixtures with minimal data analysis.

The additive $v_{8 \mathrm{a}}$ and $\mathrm{v}_{8 \mathrm{~b}}$ peak intensity for the 36 monomer mixtures shown in Figure 6B did not quantitatively correlate with the monomer composition (data not shown), contrary 
to the results for the pure monomer spectra. Overall, additional information is not gained from peak deconvolution of the phenyl ring breathing modes compared to the first derivative spectra and PLS analysis.

The peaks in the lignin spectrum may be sensitive to solvent effects, and if performed in plant tissues, the nature of the surrounding environment. In order to measure possible shifts in the phenyl ring breathing modes and to see if additional information about the monomer mixture can be measured, lignin spectra were collected in different non-aromatic solvents for which lignin has an appreciable solubility (Figure 7). The $\mathrm{S} / \mathrm{N}$ ratio varied from solvent to solvent, likely due to differences in lignin solubility. When the spectra are normalized to the most intense peak in the spectrum, few differences are observed in the spectra with the exception of lignin in $100 \mathrm{mM} \mathrm{NaOH}$. As indicated by the first derivative spectra, all peak maximums occurred at $1605.0 \pm 0.5 \mathrm{~cm}^{-1}$ except for the aqueous spectrum. Peak deconvolution also showed no shifts within the uncertainty in the phenyl ring breathing modes for 1,4-dioxane, ethanol, methanol, acetone and isopropanol. Lignin in dilute alkaline solutions is readily oxidized, and this is the likely explanation for the differences in the Raman spectrum of lignin in $100 \mathrm{mM} \mathrm{NaOH} \mathrm{[44].} \mathrm{This} \mathrm{has} \mathrm{been} \mathrm{confirmed} \mathrm{by} \mathrm{a}$ bathochromic shift and hyperchromic effect observed in the ultraviolet-visible spectrum of the alkaline lignin compared to that for the other solvents (data not shown) [8]. Given the fact that the spectra in different solvents do not provide additional details regarding the structure or composition of the lignin, it is desirable to use a nonaromatic solvent with the highest $\mathrm{S} / \mathrm{N}$ ratio for Raman measurements, which includes methanol, ethanol or 1,4 dioxane. 


\section{CONCLUSIONS}

The $1064 \mathrm{~nm}$ dispersive multichannel Raman spectrometer has performance characteristics that meet or exceed those of FT Raman spectroscopy for the analysis of lignin and model lignin monomers. The described instrument enables the collection of high quality spectra with simultaneous high spectral resolution and wide spectral coverage using a single acquisition. The described instrument will outperform alternative Raman spectrometers for the analysis of plant materials and other moderately fluorescent biological materials when robust instruments are needed, for example before plant harvest or at the biofuel processing plant. The origin of extracted lignin samples can be determined by Raman spectroscopy, and the monomer composition may be measured qualitatively using first derivative spectra and quantitatively using chemometrics. The partial least squares analysis with Raman spectroscopy offers the ability to determine more than one monomer component at a time. Current efforts are underway to use the partial least squares method for the analysis of lignin samples from diverse sources. 


\section{ACKNOWLEDGEMENTS}

This research is supported by the U.S. Department of Energy, Office of Basic Energy Sciences, Division of Chemical Sciences, Geosciences, and Biosciences through the Ames Laboratory. The Ames Laboratory is operated for the U.S. Department of Energy by Iowa State University under Contract No. DE-AC02-07CH11358. The authors thank Kevin

Langenwalter and the Department of Chemistry, Wichita State University for the use of the FT Raman instrument. 


\section{REFRENCES}

[1] S.-Y. Ding, M.E. Himmel, Journal of Agricultural and Food Chemistry, 54 (2006) 597.

[2] Y.-H.P. Zhang, L.R. Lynd, Biotechnology and Bioengineering, 88 (2004) 797.

[3] A.T. Martinez, M. Speranza, F.J. Ruiz-Duenas, P. Ferreira, S. Camarero, F.

Guillen, M.J. Martinez, A. Gutierrez, J.C. del Rio, International Microbiology, $8(2005) 195$.

[4] N.G. Lewis, E. Yamamoto, Annual Review of Plant Physiology and Plant Molecular Biology, 41 (1990) 455.

[5] D.R.D. Cyril Heitner, and John A. Schmidt (Ed.), Lignin and Lignans, CRC Press 2010, 2010.

[6] C. Saiz-Jimenez, J.W. De Leeuw, Organic Geochemistry, 10 (1986) 869.

[7] D.N. Thompson, B.R. Hames, C.A. Reddy, H.E. Grethlein, Biotechnol. Bioeng., 57 (1998) 704.

[8] S.Y. Lin, Methods in Lignin Chemistry, Spring-Verlag, 1992.

[9] K. Saito, T. Kishimoto, Y. Matsushita, T. Imai, K. Fukushima, Surf. Interface Anal., 43 (2011) 281.

[10] C. Otto, C.J. De Grauw, J.J. Duindam, N.M. Sijtsema, J. Greve, Journal of Raman Spectroscopy, 28 (1997) 143.

[11] E.A. Cutmore, P.W. Skett, Spectrochim. Acta, Part A, 49A (1993) 809.

[12] C. Cheng, T.E. Kirkbride, D.N. Batchelder, R.J. Lacey, T.G. Sheldon, J. Forensic Sci., 40 (1995) 31. 
[13] J.M. Chalmers, Everall, N. J., in: J.M. Chalmers, Griffiths, P. R. (Ed.), Handbook of Vibrational Spectroscopy, Wiley \& Sons: Chichester, New York, 2002, p. 2389.

[14] Y.Y. Huang, C.M. Beal, W.W. Cai, R.S. Ruoff, E.M. Terentjev, Biotechnology and Bioengineering, 105 (2009) 889.

[15] Y. Wang, R.L. McCreery, Anal. Chem., 61 (1989) 2647.

[16] Y.-K. Min, T. Yamamoto, E. Kohda, T. Ito, H.-o. Hamaguchi, Journal of Raman Spectroscopy, 36 (2005) 73.

[17] V.M. Hallmark, C.G. Zimba, J.D. Swalen, J.F. Rabolt, Spectroscopy (Eugene, Oreg.), 2 (1987) 40.

[18] D.R. Porterfield, A. Campion, Journal of the American Chemical Society, 110 (1988) 408.

[19] M. Fujiwara, H. Hamaguchi, M. Tasumi, Applied Spectroscopy, 40 (1986) 137.

[20] E.N. Lewis, P.J. Treado, I.W. Levin, Applied Spectroscopy, 47 (1993) 539.

[21] G.J. Gervasio, M.J. Pelletier, At-Process, 3 (1997) 7.

[22] B. Marquardt, Micro Instrumentation (2007) 211.

[23] J. Hammock, P.J. Cong, E. Bergles, W. Yang, Spectroscopy (2010) 13.

[24] C. Engert, V. Deckert, W. Kiefer, S. Umapathy, H. Hamaguchi, Applied Spectroscopy, 48 (1994) 933.

[25] C. Engert, T. Michelis, W. Kiefer, Applied Spectroscopy, 45 (1991) 1333.

[26] K. Yuzaki, H.-o. Hamaguchi, Journal of Raman Spectroscopy, 35 (2004) 1013. 
[27] S.C. Denson, C.J.S. Pommier, M.B. Denton, J. Chem. Educ., 84 (2006) 67.

[28] S. Kaminaka, T. Ito, H. Yamazaki, E. Kohda, H.-o. Hamaguchi, Journal of Raman Spectroscopy, 33 (2002) 498.

[29] M.L. Lewis, I.R. Lewis, P.R. Griffiths, Applied Spectroscopy, 58 (2004) 420.

[30] J.R. Gilchrist, J. Rebello, D. Lanzisera, J. Noonan, Laser Focus World, 36 (2000) 149.

[31] B. Chase, Y. Talmi, Applied Spectroscopy, 45 (1991) 929.

[32] J. Barbillat, E. Da Silva, J.L. Hallaert, Journal of Raman Spectroscopy, 24 (1993) 53.

[33] J. Barbillat, E. Da Silva, Spectrochim. Acta, Part A, 53A (1997) 2411.

[34] S. Barsberg, P. Matousek, M. Towrie, Macromolecular Bioscience, 5 (2005) 743.

[35] A.-M. Saariaho, A.-S. Jaaskelainen, M. Nuopponen, T. Vuorinen, Applied Spectroscopy, 57 (2003) 58.

[36] U.P. Agarwal, R.S. Reiner, Journal of Raman Spectroscopy, 40 (2009) 1527.

[37] U.P. Agarwal, Planta, 224 (2006) 1141.

[38] M. Schmidt, A.M. Schwartzberg, A. Carroll, A. Chaibang, P.D. Adams, P.J. Schuck, Biochemical and Biophysical Research Communications, 395 (2010) 521.

[39] K.L. Larsen, S. Barsberg, Journal of Physical Chemistry B, 114 (2010) 8009.

[40] C.-J. Shih, E.A. Smith, Anal. Chim. Acta, 653 (2009) 200.

[41] E.D. Schmid, R.D. Topsom, Journal of the American Chemical Society, 103 (1981) 1628. 
[42] H.J. Bernstein, G. Allen, Journal of the Optical Society of America, 45 (1955) 237.

[43] X.-F. Sun, H. Wang, G. Zhang, P. Fowler, M. Rajaratnam, Journal of Applied Polymer Science, 120 (2011) 3587.

[44] F.E. Brauns, The Chemistry of Lignin Supplement Volume, Academic Press Inc., London, 1960. 


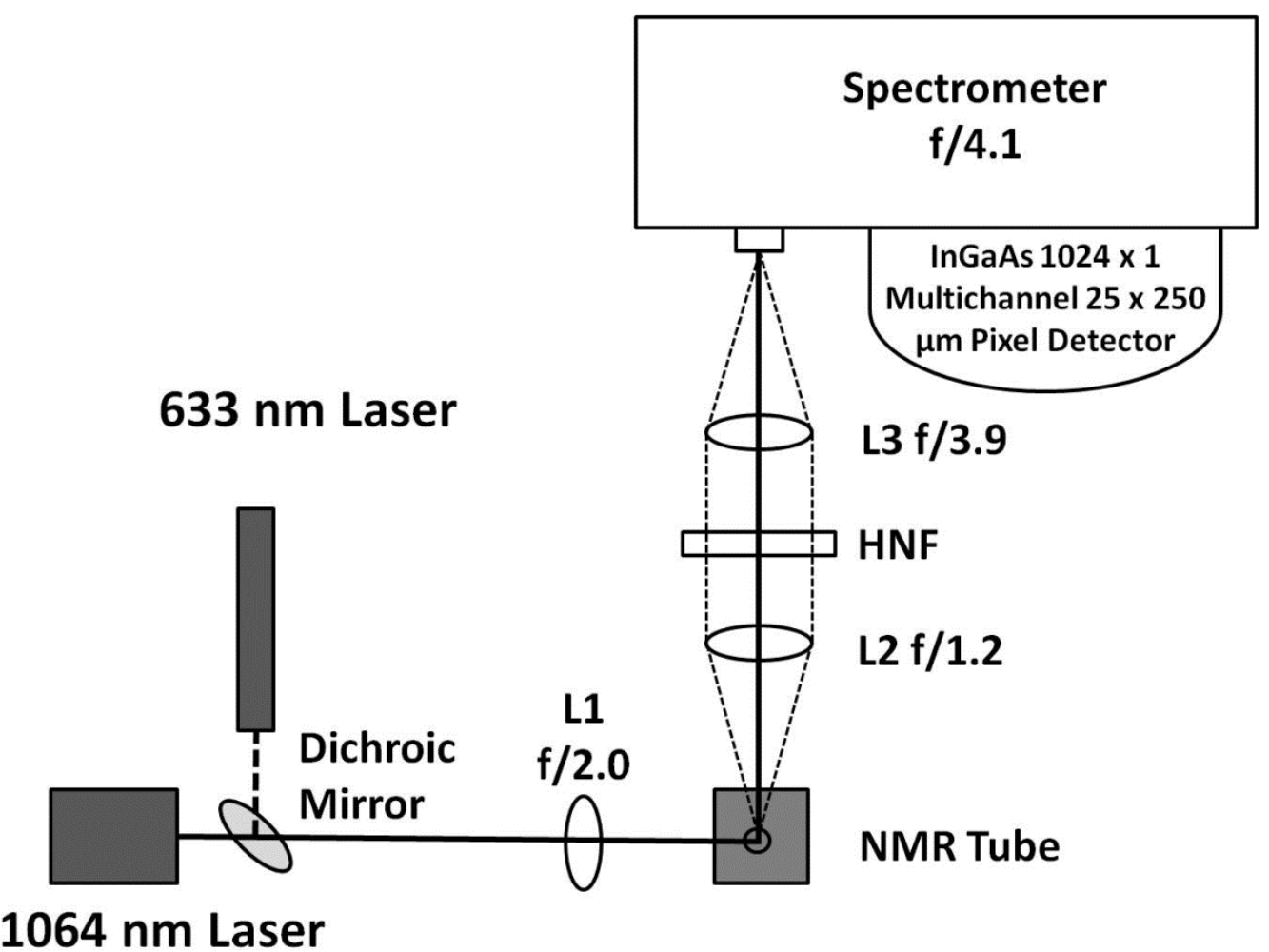

Figure 1. Instrument schematic of the $1064 \mathrm{~nm}$ dispersive multichannel Raman spectrometer. The $1064 \mathrm{~nm}$ laser is focused with a plano-convex lens (L1) onto a sample. The Raman scatter is then collected with a plano-convex lens (L2) and focused onto the spectrometer slit with a plano-convex lens (L3). A holographic notch filter (HNF) was used to filter out Rayleigh scattering. The spectrometer is equipped with a 1024-multichannel InGaAs detector. The helium-neon laser is aligned co-linearly with the $1064 \mathrm{~nm}$ laser using a dichroic mirror to aid in instrument alignment. 

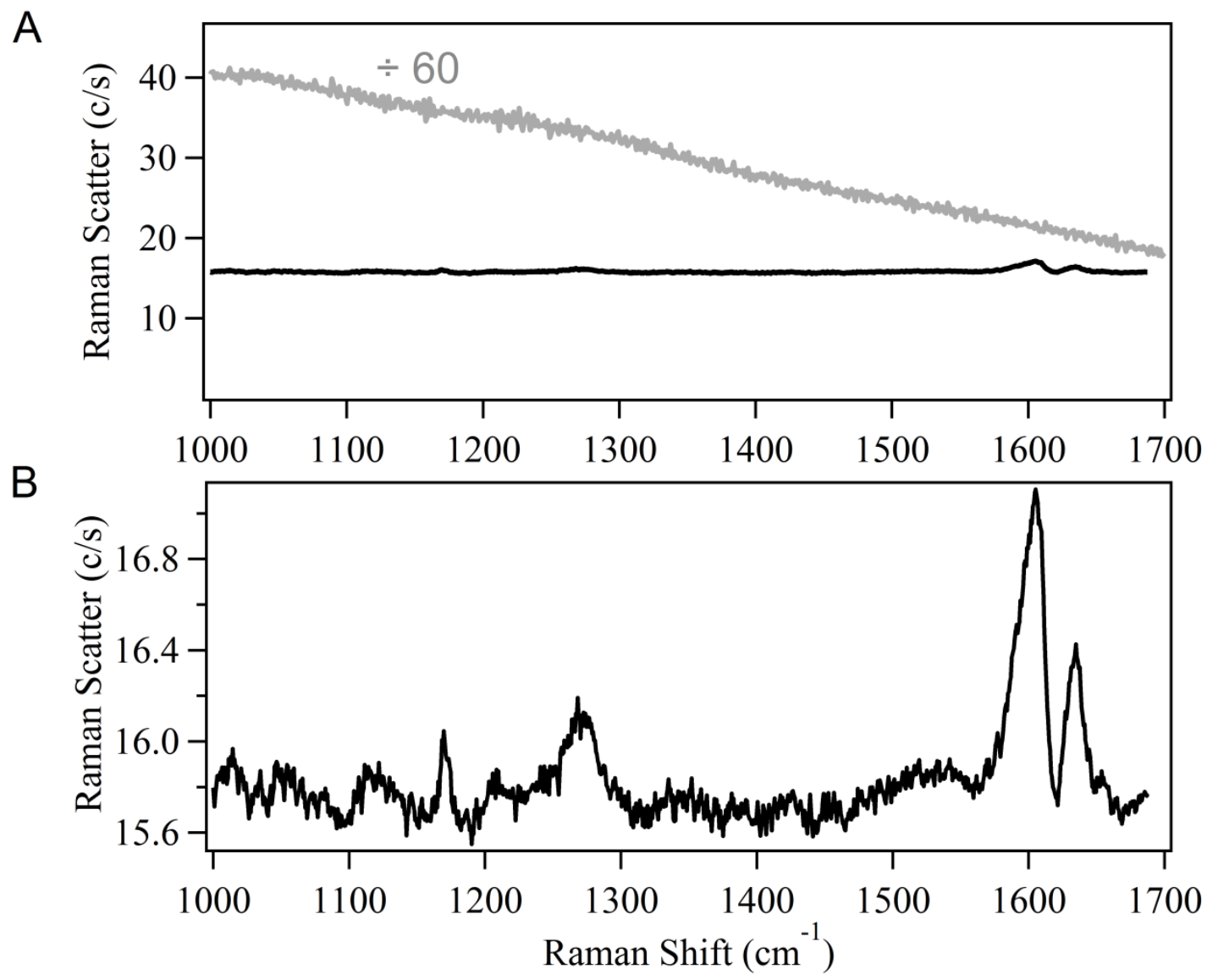

Figure 2. (A) Background subtracted Raman spectra of $50 \mathrm{mg} \mathrm{mL}^{-1}$ lignin in methanol obtained using a dispersive $785 \mathrm{~nm}$ (gray) or $1064 \mathrm{~nm}$ (black) spectrometer. The $785 \mathrm{~nm}$ excitation spectrum has been divided by 60. (B) $1064 \mathrm{~nm}$ excitation lignin spectrum shown in Figure 2A plotted on a smaller scale. For other experimental parameters see Experimental Section and Supporting Information Table S1. 


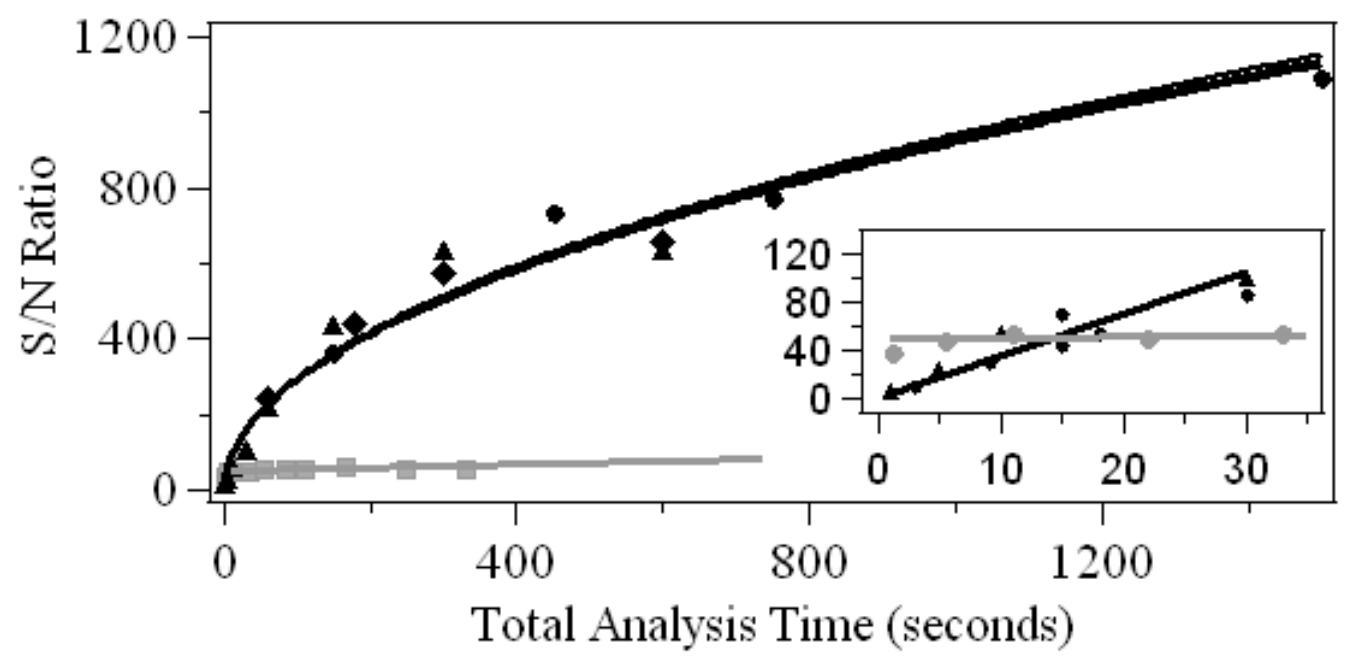

Figure 3. Plots of the measured $\mathrm{S} / \mathrm{N}$ ratios as a function of total analysis time for the FT Raman instrument (gray symbols, $480 \mathrm{mw}$ ) or the dispersive $1064 \mathrm{~nm}$ Raman instrument (black symbols, triangle varying integration times with 1 accumulation, diamond 60 second integration time and varying number of accumulations, and circle 150 second integration time with varying number of accumulations, $280 \mathrm{~mW}$ ). The data are fit (solid line) to a curve with total analysis time $\mathrm{e}^{1 / 2}$ dependence for the dispersive $1064 \mathrm{~nm}$ Raman data or a linear fit for the FT Raman data to guide the eye. Inset shows an expanded view at short total analysis times and linear fits for all data sets. 

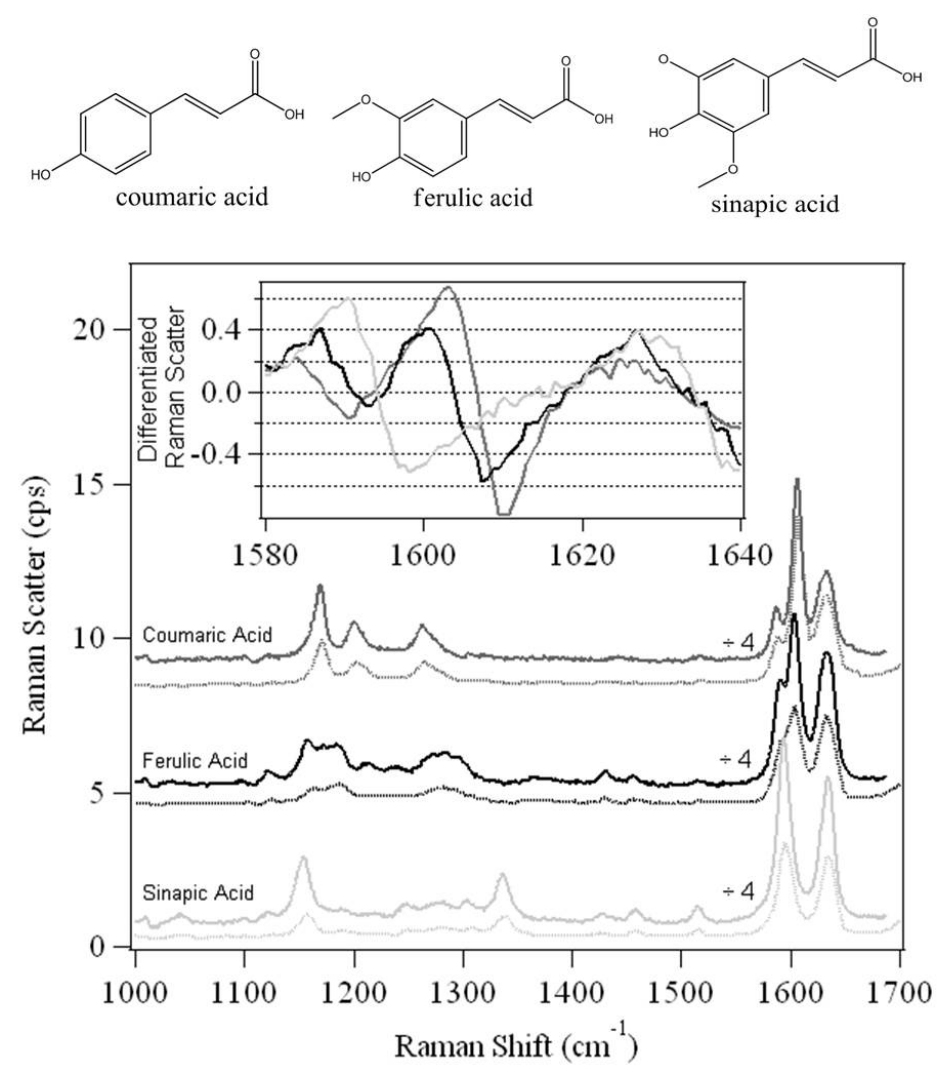

Figure 4. Raman spectra of model plant cell wall lignin monomers ferulic acid $(G)$, sinapic acid (S) and coumaric acid (H) at $50 \mathrm{mg} \mathrm{mL}^{-1}$ in 1,4-dioxane obtained with the dispersive 1064 nm Raman (solid spectra, signal divided by 4) and FT Raman (dotted spectra) instrument. Spectra have been offset for clarity. The figure insets show an expanded view of the 1580 to $1640 \mathrm{~cm}^{-1}$ first derivative spectra with all spectra overlaid (G black, S light gray, H dark gray). 




Figure 5. Raman spectra of model lignin monomers mixtures (G-S-H\%: 5-90-5 light gray; 51-40-9 dashed black; 25-25-50 thin black) overlaid with the Raman spectrum of $50 \mathrm{mg} \mathrm{mL} \mathrm{m}^{-1}$ lignin in 1,4-dioxane (thick black) obtained with the dispersive $1064 \mathrm{~nm}$ Raman instrument. 


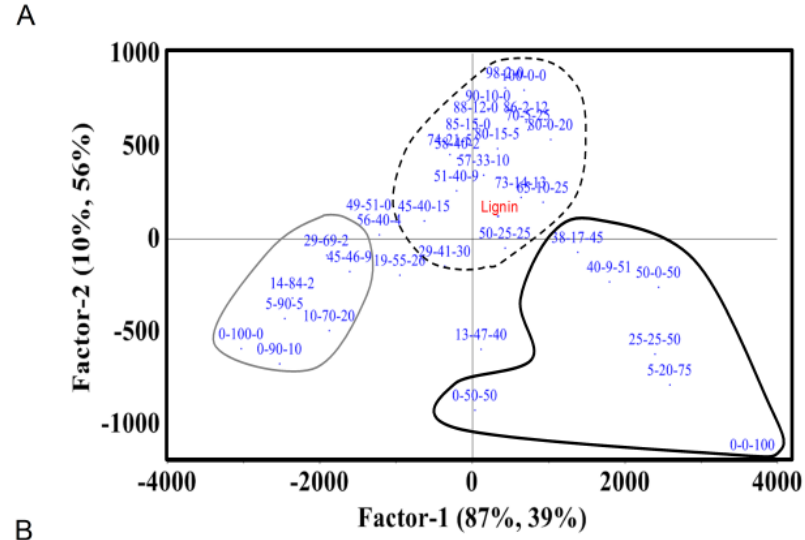

Figure 6. (A) PLS scores plot of 36 model lignin monomers, monomer mixtures and hydrolytic lignin extracted from sugarcane (red). The monomer composition is listed G-S-H. The indicated grouping is used to color code the first derivative spectra in (B). The sugarcane lignin falls in the region comprised by plant samples high in $\mathrm{G}, \mathrm{S}$ and $\mathrm{H}$. (B) First derivative Raman spectra of 33 model lignin monomers mixtures. The monomer compositions are listed from top to bottom, at $1600 \mathrm{~cm}^{-1}$, in Supplemental Information Table S4. The four monomer mixtures not circled in the PLS scores plot are omitted in Figure 6B. 


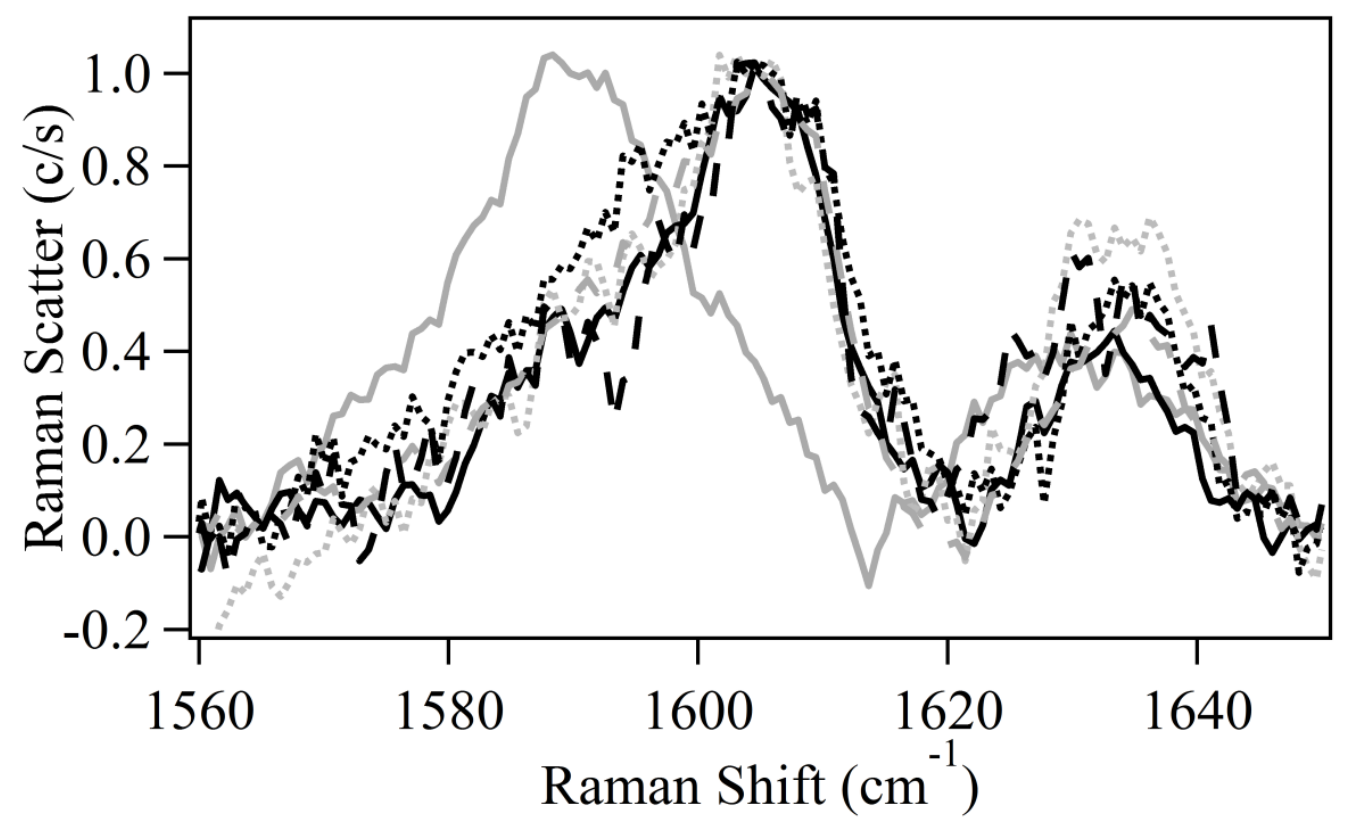

Figure 7. Raman spectra of sugarcane lignin in different solvents: $100 \mathrm{mM} \mathrm{NaOH}$ (solid gray), ethanol (solid black), 1,4-dioxane (dotted gray), acetone (dotted black), methanol (dashed gray), isopropanol (dashed black). Spectra are normalized to the highest intensity in the shown spectral region. All spectra exhibit nearly complete overlap except the spectrum in $100 \mathrm{mM} \mathrm{NaOH}$. 
Supplementary Information

\title{
$1064 \mathrm{~nm}$ dispersive multichannel Raman spectroscopy for the analysis of plant lignin
}

\author{
Matthew W. Meyer, Jason S. Lupoi and Emily A. Smith \\ ${ }^{1}$ Ames Laboratory, U.S. Department of Energy, Ames, Iowa 50011-3111, and \\ ${ }^{2}$ Department of Chemistry, Iowa State University, Ames, Iowa 50011-3111
}


Supplemental Table S1. Specifications and experimental parameters for the $1064 \mathrm{~nm}$ dispersive multichannel Raman instrument.

\begin{tabular}{|l|l|}
\hline Detector Quantum Efficiency $(\%)^{\mathrm{a}}$ & $82-85$ \\
\hline Detector Geometry & $1024 \times 1$ elements \\
\hline Detector Element Size $(\mu \mathrm{m})$ & $25 \times 250$ \\
\hline Grating Efficiency $(\%)^{\mathrm{b}}$ & \\
300 grooves/mm grating & $68-55$ \\
600 grooves/mm grating & $70-60$ \\
950 grooves/mm grating & $67-48$ \\
\hline Spectral Resolution ${ }^{\mathrm{c}} /$ Spectral Coverage $\left(\mathrm{cm}^{-1}\right)$ & \\
300 grooves/mm grating & $13 / 1715$ \\
600 grooves/mm grating & $7 / 792$ \\
950 grooves/mm grating & $4 / 411$ \\
\hline \hline Excitation Power at Sample $(\mathrm{mW})$ & 280 \\
\hline Beam Area $\left(\mu \mathrm{m}^{2}\right)$ & 95 \\
\hline Acquisition Parameters & Lignin: $600 \times 3$ \\
Integration $(\mathrm{seconds}) \mathrm{x} \#$ Accumulations & Model lignin monomers: $60 \times 3$ \\
\hline
\end{tabular}

a 1064 to $1350 \mathrm{~nm}$ (0 to $1990 \mathrm{~cm}^{-1}$ Raman Shift) spectral range

${ }^{\mathrm{b}}$ For spectral range: 600 grooves $/ \mathrm{mm}$ grating 578 to $1370 \mathrm{~cm}^{-1} ; 300$ grooves $/ \mathrm{mm}$ grating 144 to $1859 \mathrm{~cm}^{-1} ; 950$ grooves/mm grating 784 to $1195 \mathrm{~cm}^{-1}$

c $150 \mu \mathrm{m}$ slit 
Supplemental Table S2. Raman peak assignments for sinapic acid (SA), ferulic acid (FA), coumaric acid (CA), and hydrolytic lignin in 1,4 dioxane.

\begin{tabular}{|c|c|}
\hline Peak $\left(\mathrm{cm}^{-1}\right)$ & Assignment \\
\hline \multicolumn{2}{|l|}{ SA } \\
\hline 1154 & $\begin{array}{l}\mathrm{OCH}_{3} \text { Out of Phase Rock, Aromatic In Phase } \\
\text { Bend }\end{array}$ \\
\hline 1337 & Aromatic COC Stretch \\
\hline 1515 & Aromatic In Phase Bend, CO Stretch \\
\hline 1594 & Aromatic Ring Stretch $\left(\mathrm{v}_{8 \mathrm{a}} \text { and } \mathrm{v}_{8 \mathrm{~b}}\right)^{\mathrm{a}}$ \\
\hline 1634 & Substituent $C=C$ \\
\hline \multicolumn{2}{|l|}{ FA } \\
\hline 1158 & $\begin{array}{l}\mathrm{OCH}_{3} \text { Out of Phase Rock, Aromatic In Phase } \\
\text { Bend }\end{array}$ \\
\hline 1589 & Aromatic Ring Stretch $\left(\mathrm{v}_{8 \mathrm{a}}\right)$ \\
\hline 1604 & Aromatic Ring Stretch $\left(\mathrm{v}_{8 \mathrm{~b}}\right)$ \\
\hline 1633 & Substituent $\mathrm{C}=\mathrm{C}$ \\
\hline \multicolumn{2}{|l|}{ CA } \\
\hline 1168 & Hydroxyl COH Bend, Aromatic Ring Stretch \\
\hline 1200 & Methoxy In Phase Wagging \\
\hline 1263 & Aromatic Ring Bend, Methoxy CO Stretch \\
\hline 1588 & Aromatic Ring Stretch $\left(\mathrm{v}_{8 \mathrm{a}}\right)$ \\
\hline 1606 & Aromatic Ring Stretch $\left(\mathrm{v}_{8 \mathrm{~b}}\right)$ \\
\hline 1632 & Substituent $\mathrm{C}=\mathrm{C}$ \\
\hline \multicolumn{2}{|l|}{ Lignin } \\
\hline 1170 & Hydroxyl COH Bend, Aromatic Ring Stretch \\
\hline 1270 & Aromatic Ring Bend, Methoxy CO Stretch \\
\hline 1591 & Aromatic Ring Stretch $\left(\mathrm{v}_{8 \mathrm{a}}\right)$ \\
\hline 1604 & Aromatic Ring Stretch $\left(\mathrm{v}_{8 \mathrm{~b}}\right)$ \\
\hline 1634 & Substituent $\mathrm{C}=\mathrm{C}$ \\
\hline
\end{tabular}

${ }^{\mathrm{a}}$ Wilson numbering system 
Supplemental Table S3. Location of Raman peak maxima as determined by a multipeak fit of spectra for the lignin model monomers in 1,4-dioxane or lignin in the indicated solvent.

\begin{tabular}{|c|c|c|c|c|}
\hline $\begin{array}{c}\text { Lignin Model } \\
\text { Monomer/Monomer } \\
\text { Mixture }\end{array}$ & $\begin{array}{l}\text { Phenyl CC } \\
\text { Stretch } \\
\left(8 \mathrm{a}^{\mathrm{a}}, \mathrm{cm}^{-1}\right)^{\mathrm{b}}\end{array}$ & $\begin{array}{c}\text { Phenyl CC } \\
\text { Stretch }\left(8 \mathrm{~b}^{\mathrm{a}},\right. \\
\left.\mathrm{cm}^{-1}\right)^{\mathrm{b}}\end{array}$ & $\begin{array}{c}\text { Substituent } \\
\quad C=C\end{array}$ & $\begin{array}{c}\text { Other } \\
\text { Substituent }\end{array}$ \\
\hline Sinapic Acid (S) & $\begin{array}{c}1594.5 \\
\pm 0.1\end{array}$ & $\begin{array}{c}1594.5 \\
\pm 0.1\end{array}$ & $\begin{array}{c}1633.5 \\
\pm 0.1\end{array}$ & $\begin{array}{c}1154.1 \\
\pm 0.3\end{array}$ \\
\hline Coumaric Acid (H) & $\begin{array}{c}1588.6 \\
\pm 0.2\end{array}$ & $\begin{array}{l}1606.10 \\
\pm 0.05\end{array}$ & $\begin{array}{c}1632.3 \\
\pm 0.1\end{array}$ & $\begin{array}{c}1168.4 \\
\pm 0.1\end{array}$ \\
\hline Ferulic Acid (G) & $\begin{array}{c}1589.1 \\
\pm 0.2\end{array}$ & $\begin{array}{c}1603.9 \\
\pm 0.1\end{array}$ & $\begin{array}{c}1632.90 \\
\pm 0.07\end{array}$ & \\
\hline \multicolumn{5}{|l|}{$\begin{array}{c}\text { Lignin in indicated } \\
\text { solvent }\end{array}$} \\
\hline 1,4-dioxane & $\begin{array}{c}1591 \\
\pm 5\end{array}$ & $\begin{array}{c}1605.5 \\
\pm 0.8\end{array}$ & $\begin{array}{c}1633.9 \\
\pm 0.4\end{array}$ & \\
\hline Ethanol & $\begin{array}{c}1590 \\
\pm 1\end{array}$ & $\begin{array}{c}1605.2 \\
\pm 0.5\end{array}$ & $\begin{array}{c}1632.8 \\
\pm 0.3\end{array}$ & $\begin{array}{c}1168.4 \\
\pm 0.3\end{array}$ \\
\hline Methanol & $\begin{array}{c}1595 \\
\pm 2\end{array}$ & $\begin{array}{c}1606.3 \\
\pm 0.2\end{array}$ & $\begin{array}{l}1634.2 \\
\pm 0.6\end{array}$ & $\begin{array}{c}1169.7 \\
\pm 0.5\end{array}$ \\
\hline Acetone & $\begin{array}{l}1590 \\
\pm 10\end{array}$ & $\begin{array}{c}1605.5 \\
\pm 0.8\end{array}$ & $\begin{array}{l}1634.8 \\
\pm 0.6\end{array}$ & \\
\hline Isopropanol & $\begin{array}{c}1590 \\
\pm 3\end{array}$ & $\begin{array}{l}1605.9 \\
\pm 0.6\end{array}$ & $\begin{array}{l}1632.6 \\
\pm 0.5\end{array}$ & \\
\hline $\begin{array}{c}200 \mathrm{mM} \mathrm{NaOH} \\
\text { aqueous }\end{array}$ & $\begin{array}{c}1589.8 \\
\pm 0.3\end{array}$ & $\begin{array}{c}1589.8 \\
\pm 0.3\end{array}$ & $\begin{array}{l}1631.3 \\
\pm 0.6\end{array}$ & $\begin{array}{c}1167.2 \\
\pm 0.4\end{array}$ \\
\hline
\end{tabular}

\footnotetext{
${ }^{\mathrm{a}}$ Wilson numbering system notation

${ }^{\mathrm{b}}$ Bold indicates most intense peak between $\mathrm{v}_{8 \mathrm{a}}$ and $\mathrm{v}_{8 \mathrm{~b}}$
} 
Supplemental Table S4. Monomer composition of the first derivative Raman spectra from top to bottom (at $1600 \mathrm{~cm}^{-1}$ ). Numbers are percentage listed G, S, H.

\begin{tabular}{|c|c|c|}
\hline Grey & Black & Dashed Black \\
\hline $56,40,4$ & $25,25,50$ & $57,33,10$ \\
\hline $19,55,26$ & $40,9,51$ & $90,10,0$ \\
\hline $49,51,0$ & $50,0,50$ & $80,0,20$ \\
\hline $45,46,9$ & $38,17,45$ & $98,2,0$ \\
\hline $29,69,2$ & $5,20,75$ & $80,15,5$ \\
\hline $10,70,20$ & $0,50,50$ & $70,5,25$ \\
\hline $5,90,5$ & $13,47,40$ & $65,10,25$ \\
\hline $14,84,2$ & & $88,12,0$ \\
\hline $0,90,10$ & & $85,15,0$ \\
\hline & & $73,14,13$ \\
\hline & & $74,21,5$ \\
\hline & & $50,40,2$ \\
\hline & & $86,2,12$ \\
\hline & & $50,25,25$ \\
\hline & & $51,40,9$ \\
\hline & & $29,41,30$ \\
\hline & & $45,40,15$ \\
\hline
\end{tabular}




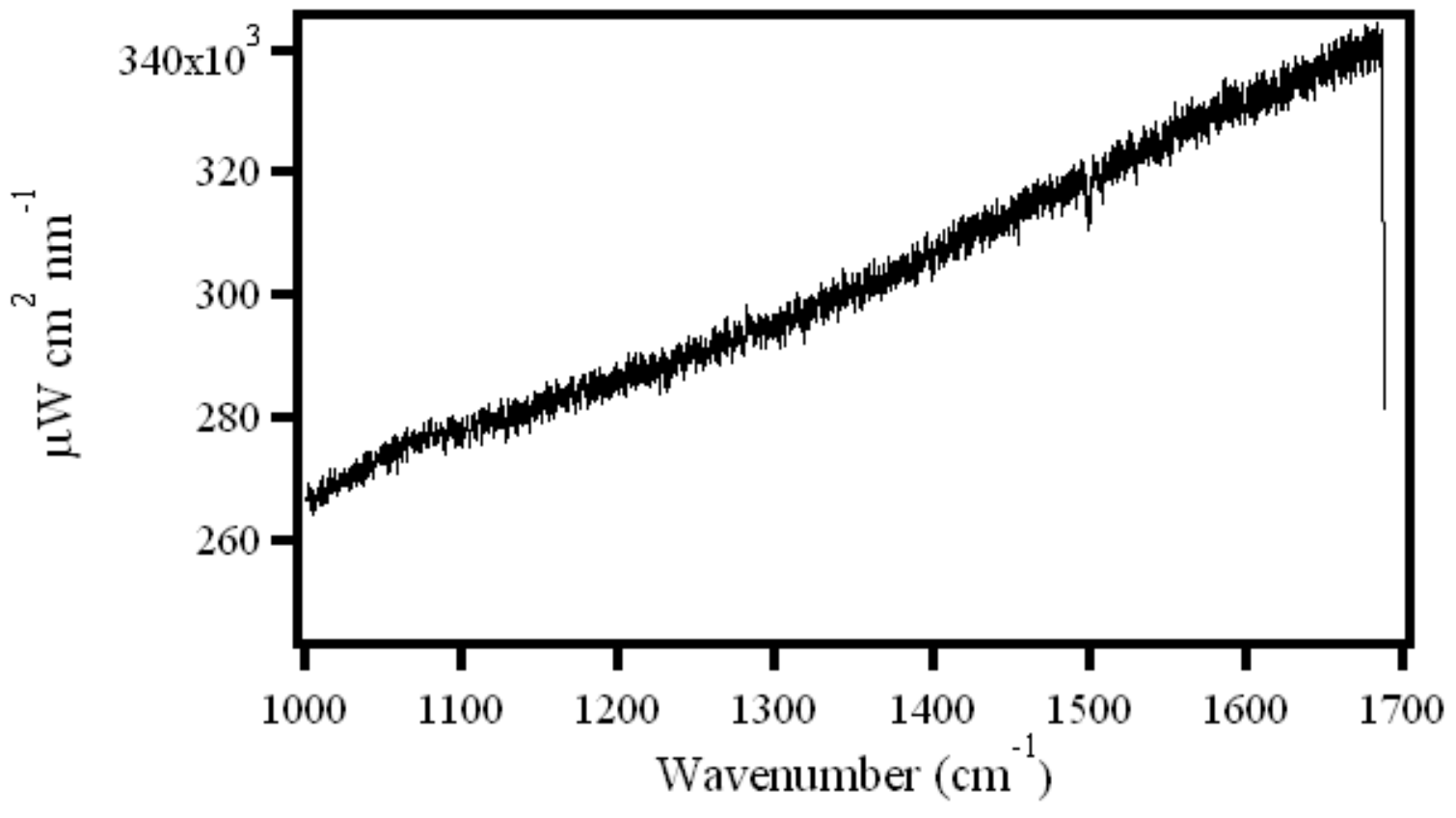

Supplemental Figure S1. Spectrum of a tungsten calibration lamp collected using the 1064 $\mathrm{nm}$ dispersive multichannel Raman spectrometer. The spectrum has been corrected for the varying lamp output in this spectral region, and can be used to intensity correct the Raman spectra for varying instrument throughputs at different wavenumbers. 


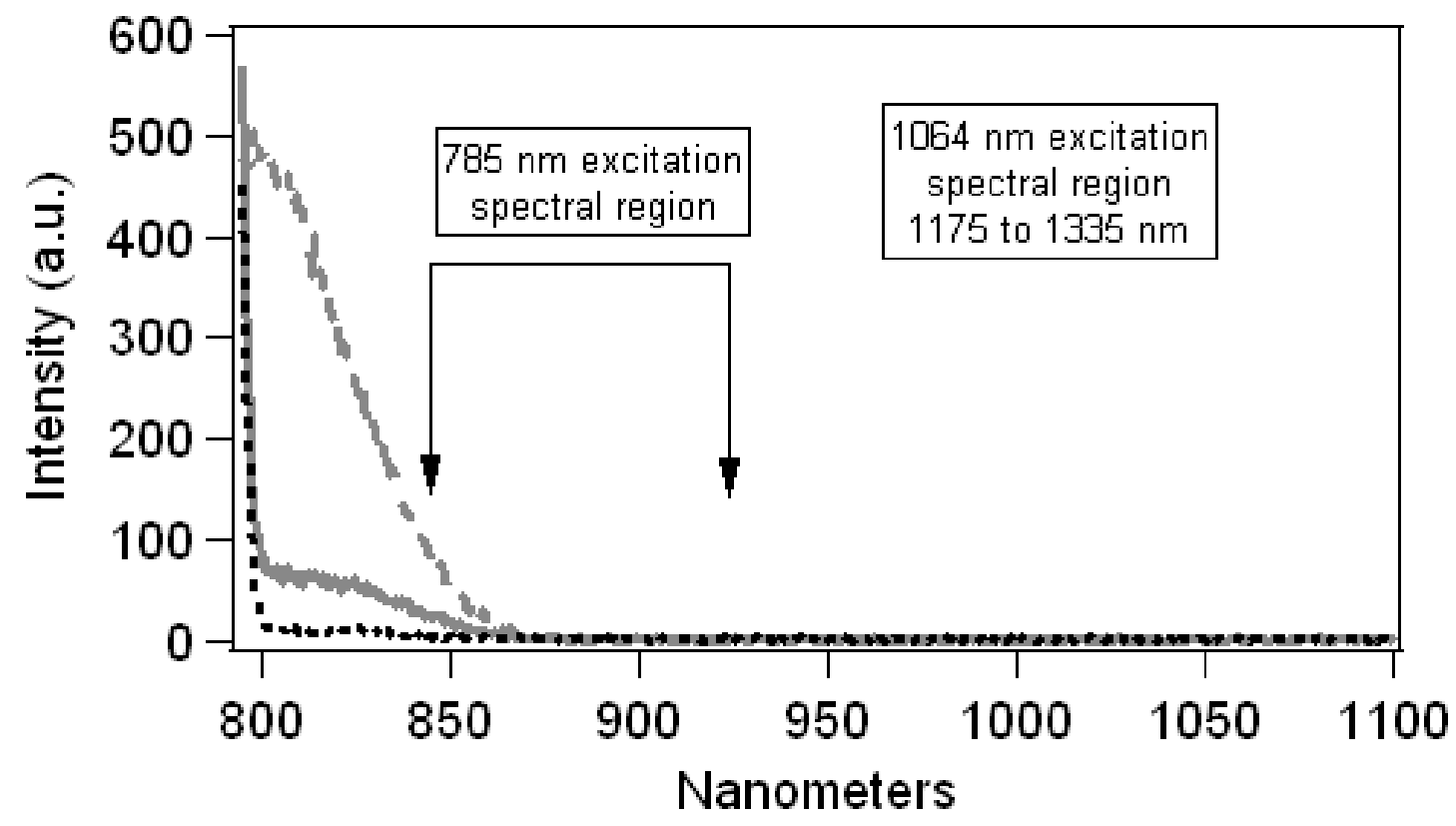

Supplemental Figure S2. Fluorescence spectrum of $200 \mathrm{mg} \mathrm{mL}^{-1}$ AlexaFlour 750 (solid black) and $50 \mathrm{mg} \mathrm{mL}^{-1}$ lignin (solid gray) and a blank spectrum of solvent (water, dotted black). Excitation wavelength: $785 \mathrm{~nm}$. 

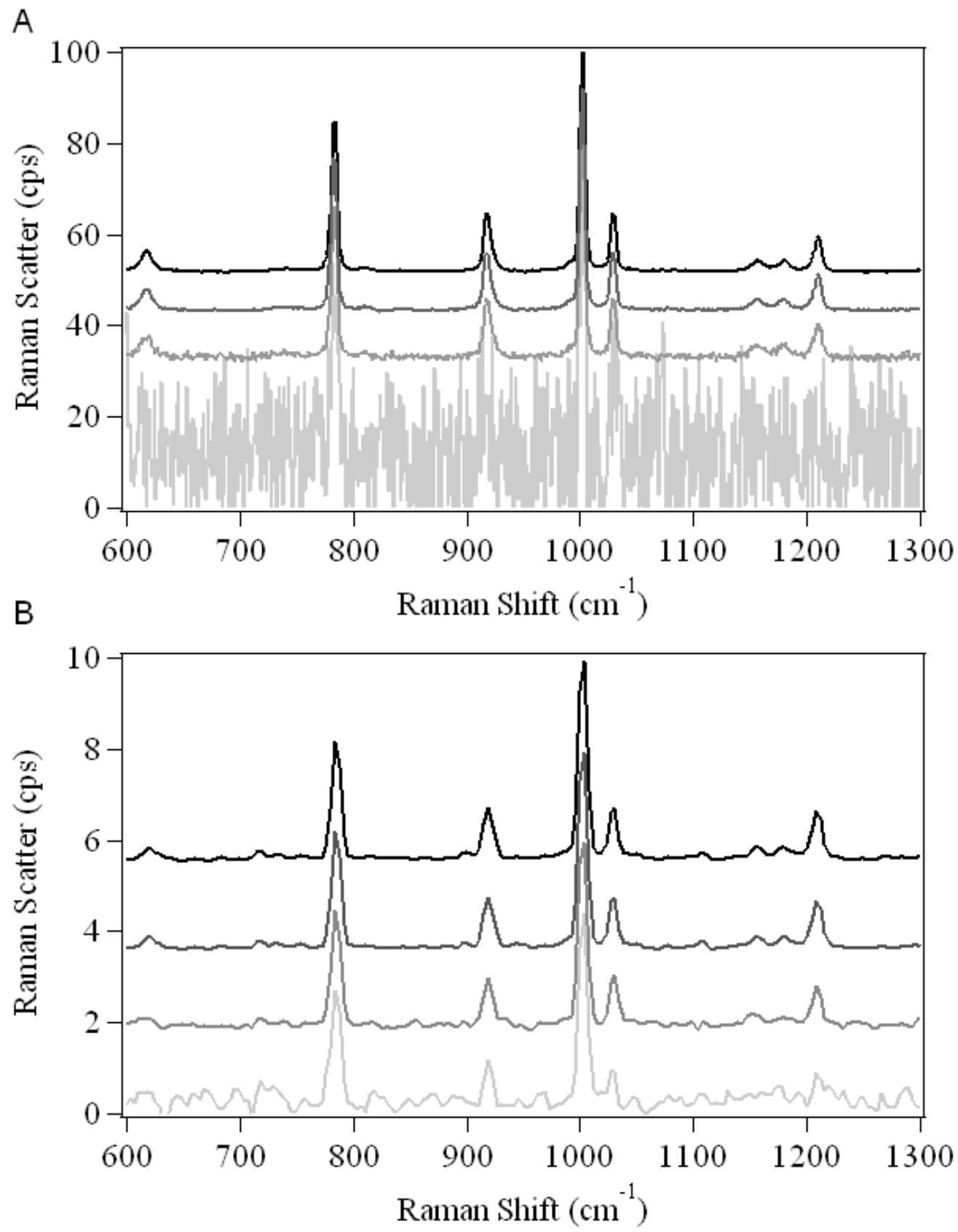

Supplemental Figure S3. Raman spectra of a toluene/acetonitrile mixture (1:1 v/v) using (A) $1064 \mathrm{~nm}$ dispersive and (B) 1064 nm FT Raman spectrometers. From bottom to top the spectra were collected with: (A) 1 second integration, 30 second integration, 150 second integration, and 300 second integration all with 1 accumulation; (B) a single scan, 10 scans, 150 scans, and 300 scans. 


\section{CHAPTER 7: GENERAL CONCLUSIONS}

Raman scatter enhancement techniques can be useful to increase the inherently weak Raman cross section especially when probing small sample volumes in submicron films. The work presented in this thesis develops novel Raman spectroscopy enhancement techniques for chemical analyses of materials at an interface. Scanning angle Raman spectroscopy has emerged as an attractive tool for obtaining label free, high signal-to-noise ratio spectra with chemical specificity for analyses of thin films and monolayers. SA-Raman spectroscopy scans an excitation beam over a prism/sample interface. The incident angle is varied between $30^{\circ}$ to $75^{\circ}$ with an angle resolution down to $0.05^{\circ}$. High angular resolution measurements allow for precise control over the depth which Raman scatter is collected from the sample under TIR. In addition the reflected light and Raman scatter can be modeled as a function of incident angle.

Research gathered for this thesis shows several interfacial configurations that have been investigated to further enhance the evanescent fields and standing waves (waveguides) to generate more Raman scatters. TIR, SPR and PWR-Raman spectroscopy can provide Raman scattering from several interfacial boundaries. In TIR-Raman spectroscopy the depth over which the signal is collected can be altered by changing the incident angle above the critical angle while in PWR-Raman spectroscopy the Raman scatter is collected from an incident angle below the critical angle. In each of these cases the information comes from a spatially localized region that can be used to increase the Raman signal and surface sensitivity in comparison to conventional Raman spectroscopy measurements. 
SA-Raman spectroscopy can make use of incident angles above and below the critical angle. Thin polymer films supporting waveguide modes were measured for thickness and composition analyses by scanning angles from $56^{\circ}$ to $70^{\circ}$, which span above and below the critical angle for a sapphire/polystyrene/air interface. The addition of a waveguide to a metal film creates a PWR-Raman spectroscopy configuration that allows for both p- and spolarizations to generate electric fields with orthogonal orientations relative to the sample. PWR-Raman spectroscopy can measure molecular orientation information not obtainable when using a gold film without a dielectric layer at the interface. PWR-Raman spectroscopy provides a reproducible Raman scattering enhancement method similar to SPR-Raman for the TIR illumination geometry. The PWR configuration also provides a decrease in the sapphire prism background signal. To show the capabilities of PWR-Raman spectroscopy, polystyrene films were spin coated onto a gold film and measured with PWR-Raman spectroscopy. The Raman peak areas were greater at the plasmon waveguide resonance angle than the surface plasmon resonance angle. The reflectivity from the sapphire/gold/polystyrene interface was also used to determine the polystyrene thickness and agreed closely with optical interferometery measurements.

This work develops a foundation for chemical measurements of numerous devices, such as photovoltaic devices that utilize thin conducting polymer films. The work will continue by addressing the following topics:

- Determining molecular order of thin film photovoltaic devices at the indium tin oxide interface as well as other interesting interfaces such as gold 
- Fabrication of SA-Raman spectroscopy sensors; such as porous silica waveguides, bi-metallic SPR and waveguide coupled SPR sensors 


\section{APPENDIX: SURFACE ENHANED RAMAN SPECTROSCOPY (SERS): RECENT DEVELOPMENTS IN SERS SUBSTRATES AND APPLICATIONS TO SINGLE CELL ANALYSIS}

A section of the review article published in Chemical Rev., 2013, DOI: 10.1021/cr300336e

Matthew W. Meyer and Emily A. Smith

Non-enhanced or conventional Raman spectroscopy is a low signal technique compared to many other molecular spectroscopies. Typical Raman cross-sections of molecules are on the order of $\sim 10^{-30} \mathrm{~cm}^{-2}$, while fluorescence and infrared cross-sections are several orders of magnitude larger. ${ }^{501}$ However, Raman scattering can be enhanced by up to 14 orders of magnitude when the Raman scatterer is in close proximity to a SERS substrate. ${ }^{501-502}$ There are two mechanisms for this enhancement: electromagnetic and chemical, which are discussed in detail elsewhere. ${ }^{503-505}$ The SERS enhancement enables detection limits that parallel fluorescence spectroscopy, down to the single molecule level. ${ }^{501,506-507}$ Short acquisition times and low laser powers can often be used for SERS as a result of the signal enhancement, ${ }^{508}$ and these conditions are favorable for live cell analyses. On the other hand, these benefits typically have to be weighed against the use of exogenous SERS substrates. 
The SERS substrate is the main component of a SERS experiment, and many variants will be described below. One common feature is noble metal substrates with roughness on the order of the wavelength of light. Although several plasmonic materials can be used as SERS substrates, Au and Ag are the most common due to good chemical stability and large SERS enhancements, respectively. ${ }^{509}$ Both solution-phase and surface-confined nanoparticle arrays can be used. Intracellular measurements usually require the delivery of nanoparticles from solution across the cell membrane since the SERS signal is generated only from molecules within a few nanometers from the SERS substrate. Surface-confined nanoparticles are suitable for measuring cell membranes and other extracellular species.

SERS has become an important tool for intracellular and extracellular measurements of chemical content. Several published reviews cover single cell applications of SERS. ${ }^{18,510-}$ ${ }^{515}$ Similar to other promising single cell analysis techniques, SERS has areas that can be improved. Careful attention must be paid to the selection of a SERS substrate for a particular application to avoid issues of low signal enhancement, poor selectivity, irreproducible signals and poor intracellular delivery to the desired cellular locations. Unlike fluorescence-based measurements where genetic approaches to specifically label a target species are in common use, SERS substrates are primarily exogenous (although intracellular synthesis routes are being pursued). ${ }^{516}$ Exogenous SERS substrates can be incorporated into the cell through cellular uptake or physical injection. ${ }^{517-520}$

In SERS experiments, any molecules within a few nanometers of the SERS substrate can generate SERS signal. The complex intracellular environments can lead to the enhancement of unwanted cellular components making the vibrational spectra difficult to 
interpret. Many of the recent developments in novel SERS substrates for cellular applications address these issues; many of these are proof of concept studies. The advancements in SERS substrates that have been applied to single cell analyses from the past two years will be covered in this section. Also select applications of SERS for the cellular detection of small molecules and cancer cells will be discussed. Excluded from the section are applications of SERS to whole tissues, ${ }^{521}$ microorganism detection and discrimination, ${ }^{522-525}$ and all nonbiological applications. ${ }^{526}$

SERS substrates: nanoparticle uptake, tracking, stability and functionalization

The first SERS study of endocytosed Au nanoparticles was performed to image an anti-cancer drug in a live cell. ${ }^{527}$ Since this first report in 1991, exogenous SERS substrates have been shown to be exceedingly dependent on the substrate uptake efficiency and localization. Important SERS substrate properties include the size, shape, surface functionality and charge. Nanoprobes or nanosensors are SERS substrates composed of a nanoparticle functionalized with a small molecule that has a large Raman cross-section, known as a Raman reporter. A detailed review of Raman reporters for biological applications has been published by Matschulat and co-workers. Recently, the work of Gregas et al. demonstrated the uptake efficiency of positively, negatively or neutrally charged nanoprobes in single cells with SERS imaging. ${ }^{528}$ The positively charged 4-aminothiophenol-labeled nanoparticles and negatively charged 4-mercaptobenzoic acid-labeled nanoparticles were taken up more readily by the cells than neutrally charged 4-thiocresol-labeled nanoparticles. The SERS spectra from three cellular locations in a macrophage cell are shown in Figure 1A and the cellular distribution of 4-aminothiophenol labeled Ag nanoparticles measured with 
SERS tracking is shown in Figure 1B. The study shows the importance of surface charge on nanoprobe uptake efficiency and stable internalization for long term observations, and can lead to advancements in the controlled delivery of exogenous SERS substrates.

The stability of nanoprobes within physiologically-relevant environments including the cell culture media and the intracellular matrix has been recently studied by Cooper et al. ${ }^{529}$ Their results show SERS probes containing a chemisorbed Raman reporter (e.g., 4mercaptobenzoic acid, 4-mercaptopyridine) on Ag nanoparticle has a stable signal inside cells for up to 24 hours, while Raman scatter from a physisorbed reporter (e.g., crystal violet, rhodamine 6G) decreased over the course of several hours. Similarly, Campbell et al. used chemisorbed 4-mercaptobenzoic acid on Au nanoshells for intracellular sensing in NIH-3T3 fibroblast cells. ${ }^{530}$ The functionalized nanoshell substrates were internalized to the cytosol through the cell's natural uptake mechanisms, and the SERS enhancements were on the order of $10^{10}$ higher compared to conventional Raman measurements that didn't utilize a SERS substrate. Another functionalized SERS substrate used alkynes as an alternative to thiolbased ligands. Pezacki et al. used alkynes to form carbon-bound Ag nanoparticles for SERS imaging of human hepatoma Huh7.5 cells. ${ }^{531}$ The alkynes produced stable nanoparticles in aqueous environments, strong SERS signals and can contain electrophilic functional groups that are incompatible with the more traditional thiolate ligands.

In order to selectively target specific components in a complex biological environment, nanoparticles can be functionalized with chemical receptors such as peptides. A functionalized nanoprobe has the ability to penetrate into the cell nucleus to detect chemical content. Recently, Hu and co-workers reported a SV-40 large T nuclear localized signal 
peptide-functionalized $\mathrm{Au}$ nanoparticle that enabled obtaining chemical content from the nucleus of single HeLa cells. ${ }^{532}$ The nuclear localization signal peptide targeted the cell nucleus without interfering Raman peaks arising from the functionalization. In other SERS experiments targeting the nucleus, Gregas et al. developed a co-functionalized SERS substrate with a nuclear targeting peptide and SERS reporter attached to a Ag nanoparticle. ${ }^{533}$ The platform allowed detection and tracking of particle uptake efficiency and localization in the nucleus with two-dimensional SERS imaging. Results show functionalization with the nuclear targeting peptide enhances uptake efficiency into the cell nucleus relative to particles without a penetrating/nuclear localization peptide.

One way to enhance signal reproducibility is to employ a SERS substrate with a fixed spatial arrangement. SERS nanopipettes have been used for in situ intracellular measurements of isolated intact HeLa human cervical carcinoma cells. ${ }^{534}$ Gogotsi et al. fabricated a nanopipette composed of a polylysine-functionalized glass capillary with a 100to 500-nm tip coated with Au nanoparticles. The positioning of the nanopipette over the cell nucleus and cytoplasm could be distinguished in HeLa cells based on the Raman spectrum. The nanopipette can be used to monitor real time intracellular biochemical processes. This was demonstrated by monitoring time resolved Raman spectra of HeLa cells after potassium chloride treatments. Further optimization of the technique could enable identification of organelles near the nanopipette and quantitative measurements of biomolecules.

Carbon nanotubes have been commonly used for cellular probes, but not traditionally used for single cell SERS measurements. ${ }^{535}$ Niu et al. developed a carbon nanotube-tipped endoscope as an alternative substrate to avoid nanoparticle aggregation. ${ }^{535}$ The $200-\mathrm{nm}$ 
carbon nanotubes were decorated with 20-nm Au nanoparticles to study intracellular environments in situ. One important feature of the carbon nanotube endoscope is the ability to enter the nucleus and allow distinction between healthy, diseased, or contaminated cells. The uniform attachment of the Au nanoparticles allowed for highly sensitive measurements of the amino acid glycine down to $\mathrm{pM}$ concentrations with good reproducibility.

The alignment of Au-coated carbon nanotube arrays with respect to the incident laser beam has recently been reported by $\mathrm{Lu}$ et al. to be a significant factor in multi-walled carbon nanotube SERS enhancements. ${ }^{536}$ Carbon nanotubes that were oriented parallel to the polarization of the incident beam had significantly larger enhancements than carbon nanotubes that were oriented perpendicular and demonstrated a detection limit of deuterated and ${ }^{13} \mathrm{C}$-labeled fatty acids on the nM level. The carbon nanotube SERS substrate was also used to measure a strain of microalgae cells to demonstrate the biological effectiveness of the SERS substrate.

Chen and co-workers compared the normal Raman and SERS signals of intracellularly-grown and passively-uptaken Au nanoparticles in nasopharyngeal carcinoma cells. ${ }^{516}$ More Raman bands could be assigned in the SERS spectra than the normal Raman spectrum of the cells. While the locations of many peaks in the spectra were the same for the intracellularly grown and passively uptaken SERS spectra, the intensities of the peaks were quite different across the two spectra. It is possible that this represents the natural variability from cell to cell, or as hypothesized by the authors that the two SERS substrates localized differently within the cell. 
Proteins, lipids and small molecules in the cell membrane can be labeled with nanoparticles. An immunolabeling protocol with Au-conjugated antibodies coupled with Ag nanoparticles for obtaining unique biomolecular information of the cellular surface was developed by Hodges and co-workers. ${ }^{537}$ The advantages of this method include better detection limits than conventional light microscopy for detecting Au nanoparticles combined with the ability to perform spectral imaging of cell membrane components.

SERS substrates: nanostars, flowers, clusters, and assemblies

Multi-branched nanoparticles such as nanostars, nanocubes and nanoflowers is an active area of interest in single cell SERS measurements due to their strong plasmon resonances in the near-infrared region (NIR) and ability to generate "hot spots" due to their sharp morphologies. ${ }^{538-539}$ SERS-encoded Au star-shaped substrates have been fabricated by Brust et al. and used for intracellular SERS imaging. ${ }^{540}$ SERS images of HeLa cells were acquired with 830-nm NIR excitation. The optical properties of the star-shaped nanoparticles in comparison to spherical shaped nanoparticles are optimum for near-infrared excitation, wavelengths that are favored to limit cell damage, and enable the Raman images to be acquired in less time.

When using multi-branched nanoparticles internalization efficiencies up to three-fold lower than spherical shaped nanoparticles may be encountered. ${ }^{540}$ One way to improve the internalization, biocompatibility and systemic retention is to encapsulate the nanoparticles with a non-toxic hydrophilic polymer. ${ }^{54}$ Recently this was done by Astilean and co-workers with a novel synthesis method for flower-shaped Au nanoparticles functionalized with a 
thiol-modified poly(ethylene) glycol (PEG) polymer. ${ }^{542}$ Intracellular detection with the multibranched flower-shaped nanoparticles was successfully tested in the cytosol of human epithelial cells with two Raman reporters: the dyes malachite green oxalate and basic fuchsin. Overall, the SERS substrate showed low toxicity and produced high signal enhancements. The PEGylated coating on the flower shaped nanoparticle can further be conjugated with specific ligands for SERS imaging and detection of cancerous cells.

SERS activity is also affected by the geometrical configuration of the SERS substrate. In a recent development, $\mathrm{Xu}$ et al. used regiospecific plasmonic nanoscale-superstructures that were assembled using DNA oligomers. ${ }^{543}$ The regiospecific nanostructures display a new level of control over the placement of nanoparticles, which can lead to structures with optimal SERS enhancements. Specific assemblies (i.e., nanorod surrounded by nanospheres) can penetrate through the HeLa cell membrane and enter the cytoplasm. They displayed adequate signal enhancement to detect lipids and potentially small molecule metabolites. These nanoprobes have the potential to be used in real-time monitoring of metabolic processes within single cells.

\section{Detection of small molecules}

Over the past few years SERS has become a useful tool for monitoring drug delivery inside cells. A novel method reported by Wang et al. used Ag nanoparticles that were preinternalized in living HeLa cells to detect the diffusion of 6-mercaptopurine and methimazole

inside a living cell. ${ }^{544}$ Metabolism rates in HeLa cells for the two drugs was monitored with a detection limit of $1 \mathrm{nM}$ for 6-mercaptopurine in a single HeLa cell, demonstrating that the 
higher background sometimes associated with SERS did not preclude measurements of compounds at physiologically relevant concentrations. Mixtures of drugs inside living cells along with kinetics and diffusion can be monitored using the pre-internalized nanoparticles. In further studies to monitor drug release, Lee et al. investigated in vitro and in vivo glutathione (GSH)-induced intracellular thiopurine anticancer drug release from $\mathrm{Au}$ nanoparticles. ${ }^{545}$ Monitoring 6-mercaptopurine and 6-thioguanine was performed with SERS in real time with drug detection limits in the nM regime. These studies demonstrate SERS ability to chemically target and track species in complex environments.

Fiber optic sensors for single cell SERS analysis can be ideal nanoprobes for in vivo measurements of single cells. ${ }^{546}$ Recently, SERS and fiber optic sensors were combined to make a submicron-sized nanoprobe to measure intracellular $\mathrm{pH}$ of a human mammary epithelial cell line and a human prostate cancer cell line. ${ }^{547}$ The intracellular $\mathrm{pH}$ of both cell lines was determined to be $\sim 7.3$, which is in the expected range for healthy cells. The fiber optic probe was physically inserted into the cells using micromanipulators and seemingly did not cause significant environmental stress while being able to capture high signal-to-noise ratio Raman spectra with 10 second exposure times and a respectable $\sim 35 \mathrm{~mW}$ of laser power. One drawback to fiber-optic probes, glass pipettes or nanopipettes is their rigid structure and relatively large sizes with respect to cell dimensions, which can cause physical damage to the cells when used for in situ measurements. ${ }^{18}$

Detection of cancer cells and treatment efficacy 
There is considerable interest in developing Raman-based methods to diagnosis cancerous cells and tissues and to monitor the efficacy of cancer therapies. Many of the strategies that have been developed to date use SERS substrates that contain a peptide or protein to target a specific cellular marker, often on the cell membrane, that is unique to cancer cells or has a differential expression in cancerous cells. ${ }^{521,548-552}$ Moskovits and coworkers developed one such strategy for distinguishing cancerous epithelium prostate cells from non-cancerous cells. ${ }^{549}$ Their approach was to ratio the signal obtained from two separate Ag nanoparticle based SERS substrates to detect the over expression of neuropilin-1 in the cancerous cell line. One substrate contained a peptide that bound to neuropilin-1 receptor on the cell membrane and a small molecule thionin Raman reporter. The other substrate contained a control cell penetrating peptide and a methylene blue Raman reporter. The excitation wavelength was in resonance with the Raman reporters. They were able to specifically detect the cancerous cells by analyzing and ratioing the signal that originated from the two different reporter molecules. The authors reported that this ratiometric approach overcomes the complications originating from differences in the focal plane, cell concentration and turbidity when imaging cell samples. Polymers and lipids have also been utilized as nanoparticle coatings for targeting cancer cells. ${ }^{553-554}$

SERS substrates have been used to monitor/increase the efficacy of therapeutic strategies. ${ }^{555-557}$ Liu et al. have developed a multi-step synthesis to make single wall carbon nanotubes coated with noble metal nanoparticles, polyethylene glycol and folic acid to target folic acid receptor positive cells. ${ }^{556}$ The nanoparticles act as both a SERS substrate and increase the efficacy of photothermal therapy used to ablate cancer cells. High SERS signals 
were recorded for the folic acid receptor containing human epidermal carcinoma cell line, but were low for the folic acid negative HeLa cell line. Overall, the nanoparticles were non-toxic in the absence of the photothermal therapy, but decreased the viability of the targeted cell line after treatment and reduced SERS imaging times from $\sim 1$ second per pixel to 0.1 second per pixel.

SERS-active substrates: dual imaging mode probes

SERS has been combined with other analytical techniques such as microfluidics, flow cytometry, fluorescence and surface plasmon resonance. ${ }^{558-566}$ SERS substrates that can act as multimodal imaging probes have been developed to target specific cancer cell lines or markers. ${ }^{567-569}$ He et al. showed that Au nanorod SERS substrates could be used for dual dark-field and SERS imaging. ${ }^{567}$ The nanorods had a Raman reporter, a polyelectrolyte coating, and finally ligands for commonly over expressed receptors on tumors (i.e., carcinoembryonic antibody or transferrin) physisorbed to the polymer coating. Dark-field imaging revealed the presence of the nanorods on HeLa cells, while the SERS imaging provided the unique Raman signal for the reporter. Thus, the SERS signal could be used for multiplexed studies to measure, for example, the expression of multiple cell surface receptors simultaneously.

A new approach recently published combined SERS with particle tracking. ${ }^{570}$ This approach not only provides the vibrational information from SERS but also the particle trajectory within the cell with sub-diffraction spatial resolution. 50-nm Au nanoparticles served as a SERS probe traveling within the cell and reporting the biochemical composition 
along its path. A combination laser-beam scanning Raman and dark-field microscope with feedback kept the beam centered on the moving nanoparticle. The molecular map of organelle transport and lysosomal accumulation were detected with $65-\mathrm{nm}$ spatial resolution and 50-ms temporal resolution. Time resolved SERS spectra obtained with a nanoparticle traveling through a macrophage cells is shown in Figure 2A. The schematic showing the relative movement of the nanoparticle while passing by intracellular molecules is shown in Figure 2B with a linear trajectory model of the Au nanoparticle shown in Figure 2C. This novel technique can capture SERS images of specific dynamic biological functions including membrane protein diffusion. Others have also combined SERS with dark field microscopy to provide information on the intracellular uptake mechanism and location in a single mammalian cell. ${ }^{571}$

Itoh and co-workers used a combination of plasmon resonance Rayleigh scattering (PRRS) and SERS with Ag nanoparticles to measure mannoproteins on yeast cell walls. ${ }^{572}$ PRRS imaging can achieve single nanoparticle detection levels, while SERS can distinguish Rayleigh scattering from the nanoparticles and other components of the cell. While a beneficial technique, it requires improvements to obtain more reproducible SERS measurements and increase both spectral and temporal resolution.

SERS spectra obtained from dual imaging modes can be used for monitoring structural and molecular information during phases of a single cell cycle. Kang et al. combined dark-field Rayleigh scatter imaging and confocal Raman microscopy for investigating different phases of a single cell during it full cell cycle. The technique has the capabilities to monitor any target cellular component during the full cell cycle using 
functionalized plasmonic nanoparticles. The technique is currently being investigated to determine if vibrational information from the cellular component can predict cell heath throughout the cell cycle process.

In summary, recent SERS developments leverage the inherent signal enhancement while improving signal specificity and reproducibility. Further advancements in new routes to intracellular delivery and fabrication of SERS substrates without loss of cell viability will expand the utility and application of SERS for single cell analyses. The novel SERS substrates reported in this review could lead to the real time analysis of cellular components, metabolic rates and drug delivery in living cells with chemical specificity. 


\section{REFERENCES}

(501) Nie, S.; Emory, S. R. Science 1997, 275, 1102.

(502) Dieringer, J. A.; Lettan, R. B., II; Scheidt, K. A.; Van Duyne, R. P. J. Am. Chem. Soc. 2007, 129, 16249.

(503) Stiles, P. L.; Dieringer, J. A.; Shah, N. C.; Van Duyne, R. P. Annu. Rev. Anal. Chem. 2008, 1,601 .

(504) Etchegoin, P. G.; Le Ru, E. C. In Surface Enhanced Raman Spectroscopy: Analytical, Biophysical and Life Science Applications; Schlücker, S., Ed.; Wiley: New York, 2011; pp 1-37.

(505) Jensen, L.; Aikensb, C. M.; Schatz, G. C. Chem. Soc. Rev. 2008, 37, 1061.

(506) Kneipp, K.; Wang, Y.; Kneipp, H.; Perelman, L. T.; Itzkan, I.; Dasari, R.; Feld, M. S. Phys. Rev. Lett. 1997, 78, 1667.

(507) Pieczonka, N. P. W.; Aroca, R. F. Chem. Soc. Rev. 2008, 37, 946.

(508) Kneipp, K.; Haka, A. S.; Kneipp, H.; Badizadegan, K.; Yoshizawa, N.; Boone, C.; Shafer-Peltier, K. E.; Motz, J. T.; Dasari, R. R.; Feld, M. S. Appl. Spectrosc. 2002, 56,150 .

(509) Sharma, B.; Frontiera, R. R.; Henry, A.-I.; Ringe, E.; Van Duyne, R. P. Mater. Today 2012, 15, 16.

(510) Xie, W.; Su, L.; Shen, A.; Materny, A.; Hu, J. J. Raman Spectrosc. 2011, 42, 1248.

(511) Vitol, E. A.; Orynbayeva, Z.; Friedman, G.; Gogotsi, Y. J. Raman Spectrosc. 2012, 43,817 .

(512) Willets, K. A. Anal. Bioanal. Chem. 2009, 394, 85.

(513) Zhang, Y.; Hong, H.; Myklejord, D. V.; Cai, W. Small 2011, 7, 3261.

(514) Li, M.; Xu, J.; Romero-Gonzalez, M.; Banwart, S. A.; Huang, W. E. Curr. Opin. Biotechnol. 2012, 23, 56.

(515) Pahlow, S.; Maerz, A.; Seise, B.; Hartmann, K.; Freitag, I.; Kaemmer, E.; Boehme, R.; Deckert, V.; Weber, K.; Cialla, D.; Popp, J. Eng. Life Sci. 2012, 12, 131. 
(516) Huang, H.; Chen, W.; Pan, J.; Chen, Q.; Feng, S.; Yu, Y.; Chen, Y.; Su, Y.; Chen, R. Spectroscopy 2011, 26, 187.

(517) Kneipp, J.; Kneipp, H.; McLaughlin, M.; Brown, D.; Kneipp, K. Nano Lett. 2006, 6, 2225.

(518) Amendola, V.; Meneghetti, M.; Fiameni, S.; Polizzi, S.; Fracasso, G.; Boscaini, A.; Colombatti, M. Anal. Methods 2011, 3, 849 .

(519) Yu, Y.; Lin, J.; Wu, Y.; Feng, S.; Li, Y.; Huang, Z.; Chen, R.; Zeng, H. Spectroscopy 2011, 25, 13.

(520) Lin, J.; Yu, Y.; Li, B.; Huang, H.; Lin, S.; Li, C.; Su, Y.; Feng, S.; Chen, G.; Li, Y.; Huang, Z.; Zeng, H.; Chen, R. Laser Phys. Lett. 2012, 9, 240.

(521) Samanta, A.; Maiti, K. K.; Soh, K.-S.; Liao, X.; Vendrell, M.; Dinish, U. S.; Yun, S.W.; Bhuvaneswari, R.; Kim, H.; Rautela, S.; Chung, J.; Olivo, M.; Chang, Y.-T. Angew. Chem. Int. Ed. 2011, 50, 6089.

(522) Chen, J.; Shen, B.; Qin, G.; Hu, X.; Qian, L.; Wang, Z.; Li, S.; Ren, Y.; Zuo, L. J. Phys. Chem. C 2012, 116, 3320.

(523) Liu, T.-Y.; Tsai, K.-T.; Wang, H.-H.; Chen, Y.; Chen, Y.-H.; Chao, Y.-C.; Chang, H.H.; Lin, C.-H.; Wang, J.-K.; Wang, Y.-L. Nat. Commun. 2011, 2, 1546.

(524) Ravindranath, S. P.; Henne, K. L.; Thompson, D. K.; Irudayaraj, J. ACS Nano 2011, 5,4729 .

(525) Wang, Y.; Ravindranath, S.; Irudayaraj, J. Anal. Bioanal. Chem. 2011, 399, 1271.

(526) Meyer, M. W.; Smith, E. A. Analyst 2011, 136, 3542.

(527) Millot, J. M.; Morjani, H.; Aubard, J.; Pantigny, J.; Nabiev, I.; Manfait, M. In Spectroscopy of Biological Molecules; Hester, R. E., Girling, R. B., Eds.; Royal Society of Chemistry: Cambridge, UK, 1991; Vol. 94; pp 305-306.

(528) Gregas, M. K.; Yan, F.; Scaffidi, J.; Wang, H.-N.; Vo-Dinh, T. Nanomed. Nanotechnol. Biol. Med. 2011, 7, 115.

(529) Sirimuthu, N. M. S.; Syme, C. D.; Cooper, J. M. Chem. Commun. 2011, 47, 4099.

(530) Ochsenkuhn, M. A.; Jess, P. R.; Stoquert, H.; Dholakia, K.; Campbell, C. J. ACS Nano 2009, 3, 3613. 
(531) Kennedy, D. C.; McKay, C. S.; Tay, L.-L.; Rouleau, Y.; Pezacki, J. P. Chem. Commun. 2011, 47, 3156.

(532) Xie, W.; Wang, L.; Zhang, Y.; Su, L.; Shen, A.; Tan, J.; Hu, J. Bioconj. Chem. 2009, 20,768 .

(533) Gregas, M. K.; Scaffidi, J. P.; Lauly, B.; Vo-Dinh, T. Appl. Spectrosc. 2010, 64, 858.

(534) Vitol, E. A.; Orynbayeva, Z.; Bouchard, M. J.; Azizkhan-Clifford, J.; Friedman, G.; Gogotsi, Y. ACS Nano 2009, 3, 3529.

(535) Singhal, R.; Orynbayeva, Z.; Kalyana Sundaram, R. V.; Niu, J. J.; Bhattacharyya, S.; Vitol, E. A.; Schrlau, M. G.; Papazoglou, E. S.; Friedman, G.; Gogotsi, Y. Nat. Nanotechnol. 2011, 6, 57.

(536) He, X. N.; Gao, Y.; Mahjouri-Samani, M.; Black, P. N.; Allen, J.; Mitchell, M.; Xiong, W.; Zhou, Y. S.; Jiang, L.; Lu, Y. F. Nanotechnology 2012, 23, 205702.

(537) Hodges, M. D.; Kelly, J. G.; Bentley, A. J.; Fogarty, S.; Patel, I. I.; Martin, F. L.; Fullwood, N. J. ACS Nano 2011, 5, 9535.

(538) Luo, Z.; Fu, T.; Chen, K.; Han, H.; Zou, M. Microchimica Acta 2011, 175, 55.

(539) Fales, A. M.; Yuan, H.-K.; Vo-Dinh, T. Langmuir 2011, 27, 12186.

(540) Rodriguez-Lorenzo, L.; Krpetic, Z.; Barbosa, S.; Alvarez-Puebla, R. A.; Liz-Marzan, L. M.; Prior, I. A.; Brust, M. Integr. Biol. 2011, 3, 922.

(541) Mandal, S.; Bonifacio, A.; Zanuttin, F.; Sergo, V.; Krol, S. Colloid. Polym. Sci. 2011, $289,269$.

(542) Boca, S.; Rugina, D.; Pintea, A.; Barbu-Tudoran, L.; Astilean, S. Nanotechnology 2011, 22, 055702.

(543) Xu, L.; Kuang, H.; Xu, C.; Ma, W.; Wang, L.; Kotov, N. A. J. Am. Chem. Soc. 2012, 134, 1699 .

(544) Yang, J.; Cui, Y.; Zong, S.; Zhang, R.; Song, C.; Wang, Z. Mol. Pharm. 2012, 9, 842.

(545) Ock, K.; Jeon, W. I.; Ganbold, E. O.; Kim, M.; Park, J.; Seo, J. H.; Cho, K.; Joo, S.W.; Lee, S. Y. Anal. Chem. 2012, 84, 2172.

(546) Vo-Dinh, T.; Zhang, Y. Wiley Interdiscip. Rev. Nanomed. Nanobiotechnol. 2011, 3, 79. 
(547) Scaffidi, J. P.; Gregas, M. K.; Seewaldt, V.; Vo-Dinh, T. Anal. Bioanal. Chem. 2009, 393, 1135.

(548) Das, A.; Soehnlen, E.; Woods, S.; Hegde, R.; Henry, A.; Gericke, A.; Basu, S. J. Nanopart. Res. 2011, 13, 6283.

(549) Pallaoro, A.; Braun Gary, B.; Moskovits, M. Proc. Natl. Acad. Sci. U.S.A. 2011, 108, 16559.

(550) Yang, J.; Wang, Z.; Zong, S.; Song, C.; Zhang, R.; Cui, Y. Anal. Bioanal. Chem. 2012, 402, 1093.

(551) Wang, X.; Qian, X.; Beitler, J. J.; Chen, Z. G.; Khuri, F. R.; Lewis, M. M.; Shin, H. J. C.; Nie, S.; Shin, D. M. Cancer Res. 2011, 71, 1526.

(552) Maiti, K. K.; Samanta, A.; Vendrell, M.; Soh, K.-S.; Olivo, M.; Chang, Y.-T. Chem. Commun. 2011, 47, 3514.

(553) Ip, S. Y.; MacLaughlin, C. M.; Mullaithilaga, N.; Joseph, M.; Wala, S.; Wang, C.; Walker, G. C. Proc. SPIE 2012, 8212, 821204.

(554) Tam, N. C. M.; Scott, B. M. T.; Wilson, B. C.; Zheng, G. Proc. SPIE 2011, 8090, 80900A.

(555) El-Said, W. A.; Kim, T.-H.; Kim, H.; Choi, J.-W. Biosens. Bioelectron. 2011, 26, 1486.

(556) Wang, X.; Wang, C.; Cheng, L.; Lee, S.-T.; Liu, Z. J. Am. Chem. Soc. 2012, 134, 7414.

(557) Balint, S.; Rao, S.; Marro, M.; Miskovsky, P.; Petrov, D. J. Raman Spectrosc. 2011, 42,1215 .

(558) Connatser, R. M.; Cochran, M.; Harrison, R. J.; Sepaniak, M. J. Electrophoresis 2008, 29, 1441.

(559) Deckert, V. J. Raman Spectrosc. 2009, 40, 1336.

(560) Potara, M.; Gabudean, A. M.; Astilean, S. J. Mater. Chem. 2011, 21, 3625.

(561) Meyer, S. A.; Le Ru, E. C.; Etchegoin, P. G. Anal. Chem. 2011, 83, 2337.

(562) Syme, C. D.; Sirimuthu, N. M. S.; Faley, S. L.; Cooper, J. M. Chem. Commun. 2010, $46,7921$. 
(563) Nolan, J. P.; Duggan, E.; Liu, E.; Condello, D.; Dave, I.; Stoner, S. A. Methods 2012, $57,272$.

(564) Qian, J.; Jiang, L.; Cai, F.; Wang, D.; He, S. Biomaterials 2011, 32, 1601.

(565) Wang, Y.; Chen, L.; Liu, P. Chem. Eur. J. 2012, 18, 5935.

(566) Wang, Z.; Wu, H.; Wang, C.; Xu, S.; Cui, Y. J. Mater. Chem. 2011, 21, 4307.

(567) Jiang, L.; Qian, J.; Cai, F.; He, S. Anal. Bioanal. Chem. 2011, 400, 2793.

(568) Olivo, M.; Lucky, S. S.; Bhuvaneswari, R.; Dendukuri, N. Proc. SPIE 2011, 8087, $80870 \mathrm{~T}$.

(569) Wang, Z.; Zong, S.; Yang, J.; Li, J.; Cui, Y. Biosens. Bioelectron. 2011, 26, 2883.

(570) Ando, J.; Fujita, K.; Smith, N. I.; Kawata, S. Nano Lett. 2011, 11, 5344.

(571) Park, J.-H.; Park, J.; Dembereldorj, U.; Cho, K.; Lee, K.; Yang, S. I.; Lee, S. Y.; Joo, S.-W. Anal. Bioanal. Chem. 2011, 401, 1635.

(572) Syamala, K. M.; Abe, H.; Fujita, Y.; Tomimoto, K.; Biju, V.; Ishikawa, M.; Ozaki, Y.; Itoh, T. Langmuir 2012, 28, 8952. 

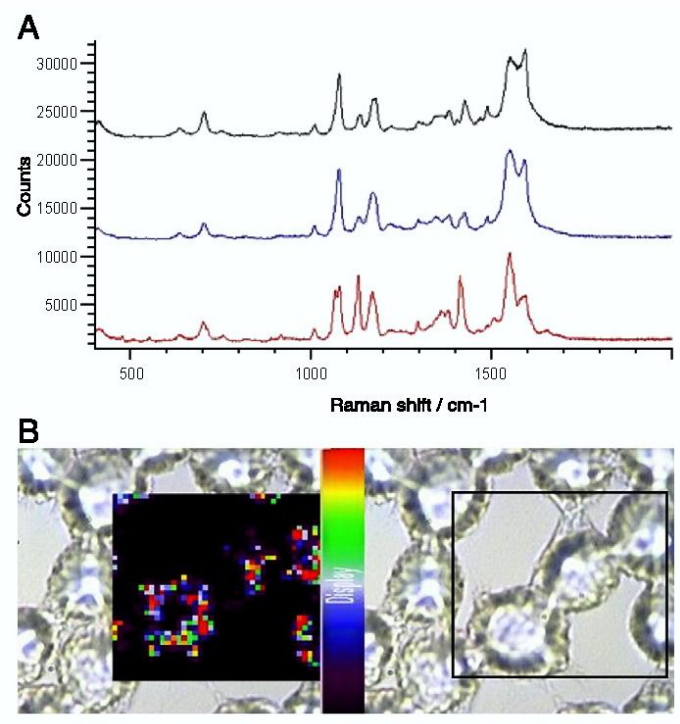

Figure 1. Monitoring the uptake of SERS nanoprobes. (A) SERS spectra of 4aminothiophenol sampled over three different locations in a macrophage cell. (B) Cellular distribution of the negatively charged 4-aminothiophenol labeled Ag nanoparticles in macrophage cells using SERS mapping. Reprinted with permission from ref 528. Copyright 2011 Elsevier B.V. 


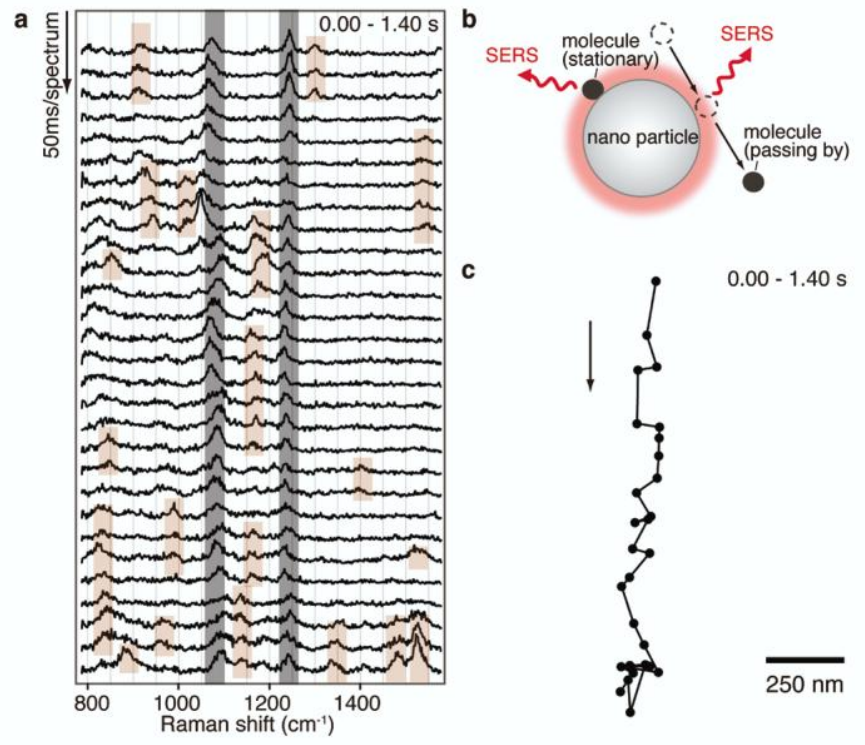

Figure 2. Dynamic SERS imaging inside a living cell can provide local molecular information over millisecond intervals. (A) High temporal resolution Raman spectra collected at $50 \mathrm{~ms}$ intervals. The stationary Raman peaks indicate the nanoparticle had prolonged interaction with the molecule and Raman peaks appearing and disappearing indicate molecules short residence times. (B) Schematic of the nanoparticle with molecules passing by and molecules with prolonged interactions. (C) Example linear trajectory of a Au nanoparticle acquired during a dynamic SERS imaging experiment. Reprinted with permission from ref 570. Copyright 2011 American Chemical Society. 